\title{
The Electrothermal Instability on Pulsed Power Ablations of Thin Foils
}

\author{
by \\ Adam M. Steiner \\ A dissertation submitted in partial fulfillment \\ of the requirements for the degree of \\ Doctor of Philosophy \\ (Nuclear Engineering and Radiological Sciences) \\ in the University of Michigan \\ 2016
}

Doctoral Committee:

Professor Ronald M. Gilgenbach, Chair

Professor John E. Foster

Assistant Research Scientist Nicholas M. Jordan

Professor Mark J. Kushner

Professor Yue Y. Lau 


\section{DEDICATION}

To my mother 


\section{ACKNOWLEDGEMENTS}

This work could not have happened without vital assistance and input from many talented and dedicated individuals. First, I would like to thank my advisor, Professor Ronald Gilgenbach, for recruiting me into a lab with exciting research opportunities and great camaraderie among colleagues. His guidance throughout graduate school has been indispensable to my progress as a student and as a scientist, and I have observed firsthand the difference a compassionate advisor like "Prof G" can have on a grad student's overall well-being. Together with Professor Y.Y. Lau, Professor Gilgenbach has built a research group that is truly top-notch in pulsed power plasma science. Professor Lau has also been a great mentor, sharing his theoretical expertise in meetings, group lunches, and of course happy hours. I am grateful to Professor John Foster for always having an open door for discussing ideas for experiments (and for the occasional racquetball match after work), and I am very fortunate to have him on my committee. Thanks also go to Professor Mark Kushner, an excellent teacher of plasma physics who also provided valuable insights to help develop my thesis research as my cognate committee member. Of course, I also wish to thank Dr. Nick Jordan, who despite his many duties as our lab manager always found time to help with data collection and analysis. Finally, while our overlap at the University of Michigan was brief, Professor Ryan McBride offered his help from the day he walked in the door to our lab, and his input is greatly appreciated.

Other scientists who were instrumental to my research include Dr. Tom Awe and Dr. Kyle Peterson of Sandia National Labs, who met with me at conferences and over the phone and 
email to discuss past research and ideas for new experiments. Particular thanks go to Dr. Jacob Zier, a former graduate student in our lab who attempted the daunting task of teaching me how to run the LTD in only six months as he wrote his own dissertation. Thanks also go to Dr. Matthew Gomez, whom I also briefly overlapped with as a graduate student and who has provided advice on numerous occasions. Mark Perrault provided indispensable support to my research as our lab electrician, machinist, and safety officer; he will be pleasantly surprised to learn I still have two functional legs despite the number of times I wore shorts to the lab. A very special thank you goes to Professor Steven Shannon of North Carolina State University, who brought me into his lab as an undergraduate, ignited my passion for plasma physics, and introduced me to Professor Gilgenbach at a conference.

Perhaps the most indispensable contributors to the day-to-day research required for this thesis were my fellow graduate students, who are all both colleagues and close friends. Dr. Sonal Patel is both a brilliant scientist and everyone's favorite random word generator. David Yager-Elorriaga and I looked out for each other's sanity as we learned how much fun a full LTD rebuild can be. Steven Exelby invigorates everyone who works in our lab with his burning passion for social justice. Paul Campbell, although he arrived late into my grad school career, was pretty much the only reason I was able to finish collecting data while frantically writing my thesis as the deadline loomed over me. It has also been a great privilege to get to know and work with Geoff Greening. I also thank the students of Professor Lau, particularly Professor Peng Zhang, Dr. Ian Rittersdorf, Dr. David Simon, Dr. Matthew Weis, for all their help and friendship. Many undergraduate students contributed to research in our lab; Jeremy Policht and Andrew Denniston were particularly helpful in collecting data for my dissertation. 
Words cannot express my gratitude to my mother, Stacia, who raised me and shaped me into the person I am today. Thank you, Mom, for always being there for me; for sacrificing whatever it took to ensure I got a good education; for all the support and advice (solicited or otherwise); and for always doing your best to make sure I was happy. Know that I will forever appreciate you and that I love you. To my beautiful fiancée, Kierstin, I thank you for all your support during what was probably the most stressful part of my life. I am so happy that you agreed to spend the rest of your life with me. I do not know if I could have made it through the end of grad school without your love and encouragement. Last but most importantly, I thank God for putting all these wonderful people in my life. I would not be here without His hand guiding me, and with His gifts I hope to make contributions through my work that make the world a better place.

During my graduate studies, I was supported in part by a Rackham Graduate fellowship and by a National Physical Science Consortium (NPSC) fellowship funded by Sandia National Laboratories. Research presented in this dissertation was supported by the NNSA under DOE Cooperative Agreement DE-NA0001984, the U.S. DOE through award DE-SC0012328, Sandia National Laboratories, and the National Science Foundation. The fast framing camera was supported by a DURIP, AFOSR Grant \#FA9550-15-1-0419. 


\section{TABLE OF CONTENTS}

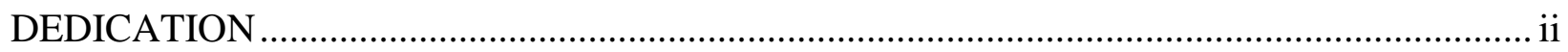

ACKNOWLEDGEMENTS ..........................................................................................

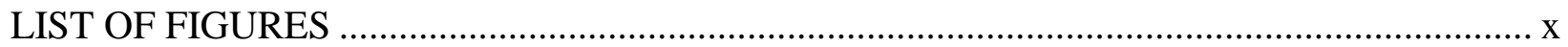

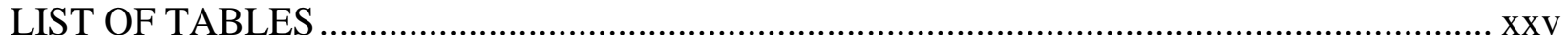

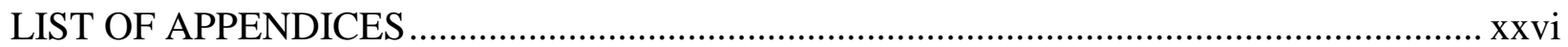

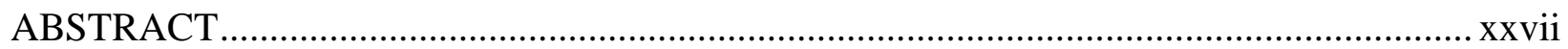

\section{CHAPTER}

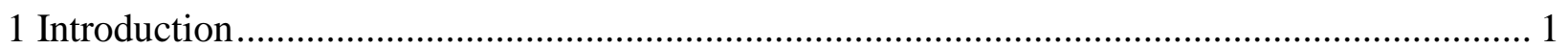

2 Theory of the Electrothermal Instability ..................................................................... 5

2.1 Qualitative Description of ETI.................................................................. 5

2.2 Derivation of expansionless ETI growth rate from perturbation analysis ............. 10

2.3 Characteristics of ETI growth ............................................................... 14

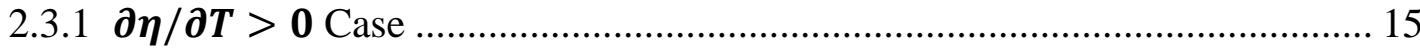

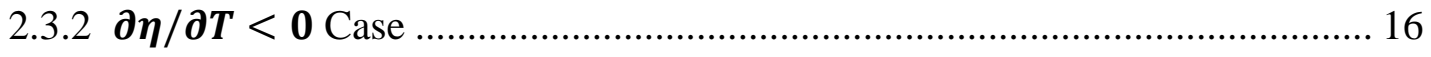

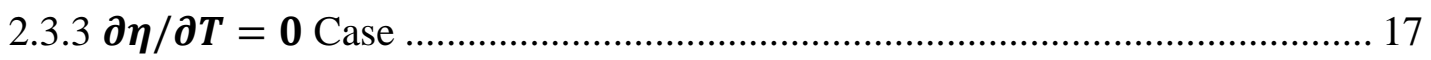

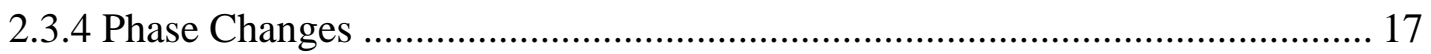


2.4 Calculated Growth Rates and Instability Conditions.

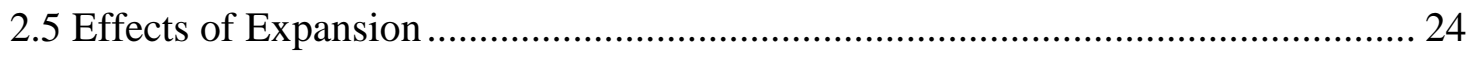

3 Experimental Configuration for Foil and Liner Ablations ............................................... 26

3.1 Planar Foil Ablations on Atmospheric Pulsed Power Discharges ........................ 26

3.1.1 Pulsed power system and diagnostics ................................................. 26

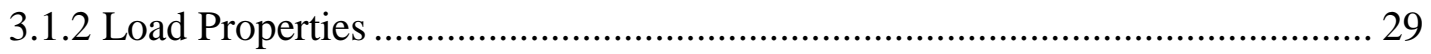

3.1.3 Experiments Utilizing Gas Flows or Vacuum ....................................... 31

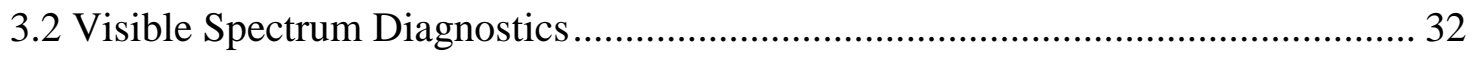

3.2.1 Ultrafast Framing Camera Imaging System .......................................... 32

3.2.2 Time-Integrated Spectroscopy and Time-Resolved Emission Measurements 35

3.3 Time-Resolved Temperature Measurements ................................................ 37

3.4 Cylindrical Liner Implosions and Explosions.............................................. 42

4 Analysis of the MAIZE Linear Transformer Driver with Dynamic Loads ........................... 45

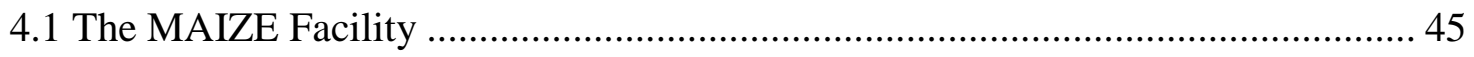

4.2 Standard Operation MAIZE Circuit Model ................................................... 47

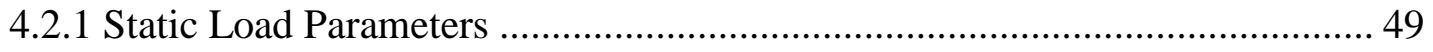

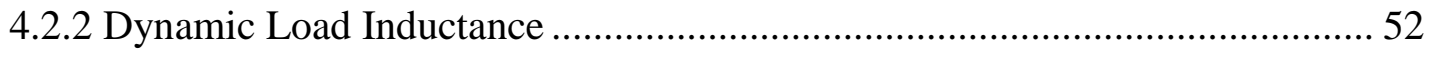

4.3 Circuit Model with Variable Switch Timings.................................................. 54

4.4 Inductance Measurements on Planar Wire Array Implosions ............................. 58

4.4.1 Planar Wire Array Setup and Diagnostics ................................................. 58 
4.2.2 Calculated Inductance Results 60

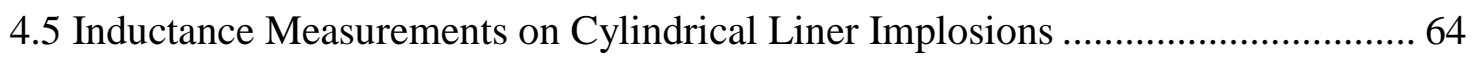

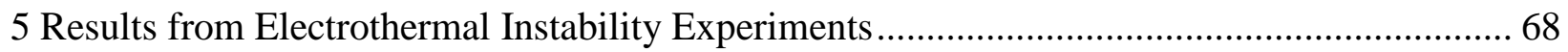

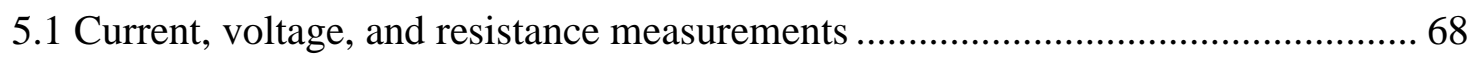

5.2: Visible Self-Emission Measurements ........................................................ 72

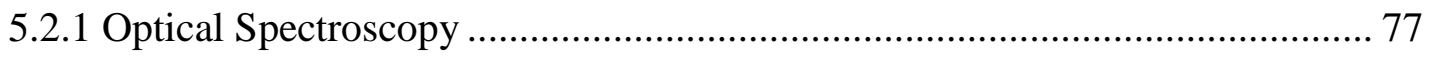

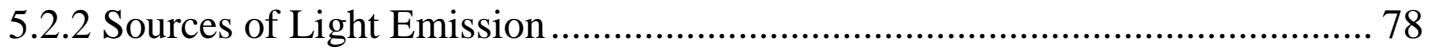

5.2.3 Comparison with $\mathrm{SF}_{6}$ and Vacuum Backgrounds.................................... 81

5.3: Temperature Measurements of Ablating Foils ................................................. 86

5.4 Analysis of Instability Spatial and Temporal Growth ........................................ 93

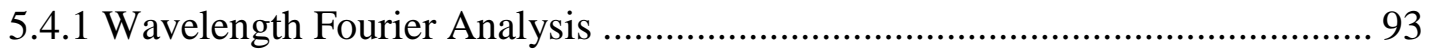

5.4.2 Growth Rate Measurements ................................................................ 100

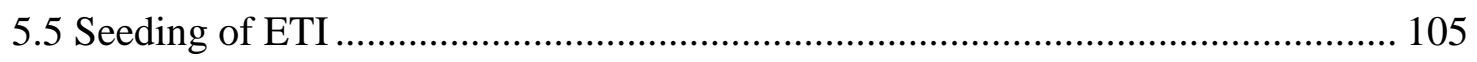

5.6 Formation of Filamentation ETI …...................................................... 110

6 Seeding of Hydrodynamic Instabilities from the Electrothermal Instability on Liner Ablation

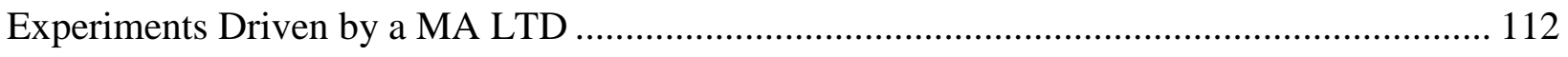

6.1: Material Dependence of Instability Growth ............................................... 112

6.2.1: MRT Instability on Plastic-Backed Aluminum and Titanium Foils ............. 118

6.2.2: Sausage Instability on Aluminum and Tantalum Foils............................ 122

6.2: Effects of Dielectric Coating on the Sausage Instability ............................... 127 


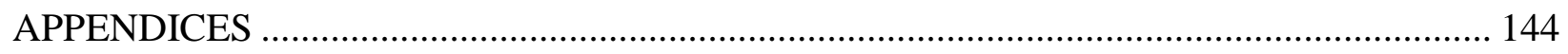

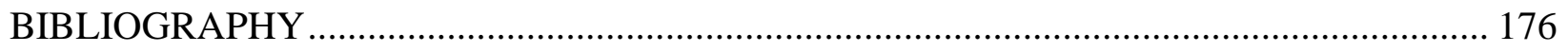




\section{LIST OF FIGURES}

Figure 2.1: Series combination of $\mathrm{N}$ resistors with temperature dependent resistivity. A temperature perturbation applied to any resistor in the circuit causes positive feedback if $\partial \eta / \partial T>0$ and negative feedback if $\partial \eta / \partial T<0$

Figure 2.2: Parallel combination of $\mathrm{N}$ resistors with temperature dependent resistivity. A temperature perturbation applied to any resistor in the circuit causes positive feedback if

$\partial \eta / \partial T<0$ and negative feedback if $\partial \eta / \partial T>0$ 8

Figure 2.3: Illustration of the electrothermal instability. (a) Material with positive $\partial \eta / \partial T$ tends to grow striations of hotter, more resistive material, shown in red, with $k$ parallel to $J$. This is analogous to the series circuit described in Fig. 2.1. (b) Material with negative $\partial \eta / \partial T$ tends to grow filamentations of hotter, less resistive material, shown in red, with $k$ perpendicular to $J$. This is analogous to the parallel circuit described in Fig. 2.2...... 9

Figure 2.4: Self-correlation ETI. (a) Striation-form, current carrying material with $\partial \eta / \partial T>0$. An initial hotspot growing from nonuniformities in resistivity becomes increasingly resistive due to locally increased Ohmic heating (left). Current attempts to flow around the hotspot, bunching the current density along the edges of the hotspot, which leads to areas of increased Ohmic heating on either side of the hotspot (center). This process expands the hotspot in the direction perpendicular to current until the hotspot has become a striation (right). (b) Filamentation form, current carrying material with $\partial \eta / \partial T<0$. An initial hotspot is less resistive, drawing more current into the hotspot (left). This causes current bunching above and 
below the hotspot, increasing the Ohmic heating in these regions, which expands the hotspot vertically (center). This process continues in the direction parallel to current until the hotspot has become a filament. (right)

Figure 2.5: Problem geometry for ETI growth in a planar foil. Foil thickness $d$ is along the $x$-direction and current travels along the $z$-direction with AK gap spacing $L$. The foil is considered electrically thin $(d<<\delta)$. 12

Figure 2.6: (a) Temperature of aluminum at atmospheric pressure as a function of input enthalpy $\Delta H$, where the zero of input enthalpy has been defined at room temperature, plotted up to complete vaporization at $13 \mathrm{MJ} / \mathrm{kg}$. (b) Specific heat capacity as a function of temperature. Since the foil spends very little time in the solid phase, the solid heat capacity has been approximated as constant at the average value in the interval ( $300 \mathrm{~K}, 933 \mathrm{~K})$. Note that the heat capacity is not defined during a phase change. Empirical fits are from Reference [26]. 20

Figure 2.7: Empirical fit of solid and liquid aluminum density at atmospheric pressure (a) as a function of enthalpy and (b) as a function of temperature. Empirical fits are from Reference [26].

Figure 2.8: Empirical fit of solid and liquid aluminum resistivity (a) as a function of enthalpy and (b) as a function of temperature. Empirical fits are from Reference [26]. 21

Figure 2.9: Solid and liquid aluminum thermal conductivity (a) as a function of enthalpy and (b) as a function of temperature from References [29] and [30]. At temperatures greater than $1000 \mathrm{~K}$, thermal conductivity was calculated from resistivity using the Weideman-Franz Law [31]. 
Figure 2.10: Empirical fit of solid and liquid aluminum effective resistivity of a fixed foil section, accounting for thermal expansion, plotted against (a) enthalpy and (b) temperature. Empirical fits are from Reference [26] 22

Figure 2.11: Growth rates for various values of k, calculated from equation (2.18) for a 4 $\mathrm{kA}, 600 \mathrm{~ns}$ risetime pulse into a $800 \mathrm{~nm}$ x $9.0 \mathrm{~mm}$ x $7.0 \mathrm{~mm}$ aluminum foil. Experimentally measured current and voltage were used to calculate energy delivered to the foil, which was used with equations (2.36) through (2.39) to calculate current density, resistivity, specific heat, density, and thermal conductivity as a function of time for the bulk (unperturbed) foil. 23

Figure 2.12: Minimum conditions for ETI growth obtained for a 4 kA, 600 ns risetime pulse into a $800 \mathrm{~nm}$ x $9.0 \mathrm{~mm}$ x $7.0 \mathrm{~mm}$ aluminum foil. Current density, resistivity, specific heat, density, and thermal conductivity were obtained as in Figure 2.11. These values were input into equations (2.21) and (2.23) to obtain $\lambda \min$ and $\alpha \min$

Figure 3.1: Pulse power generator. (A) $240 \mathrm{nF}$ capacitor (B) Atmospheric pressure air spark gap switch (C) $0.83 \Omega$ resistor array (D) High voltage input from power supply. The applied high voltage is slowly increased to $13 \mathrm{kV}$, at which point the spark gap switch spontaneously breaks down and conducts current.

Figure 3.2: Schematic of the pulsed power circuit. Voltage across the load is monitored with the two North Star high voltage probes indicated on the diagram. Current is measured using a Pearson coil placed around the output wire. The load inductance is negligible compared to the high inductances of the circuit; load resistance is measured assuming zero load inductance from the voltage and current measurements. 28

Figure 3.3: (a) Load hardware for atmospheric foil ablations. (A) Connection to pulser output (B) connection to pulser ground side (C) Connection to high voltage probe (D) Foil load. 
Conventional current flows from left to right.(b) Schematic view of foil with dimensions indicated as referred to in the text.

Figure 3.4: Current density distribution calculated from the 1-D method of moments for a thin conducting foil. The calculation was performed for a $600 \mathrm{~ns}$ risetime pulse into an $800 \mathrm{~nm}$ x $7 \mathrm{~mm}$ room temperature aluminum foil with resistivity $2.7 \times 10^{8} \Omega-\mathrm{m}$. For comparison, a uniform current density into the same cross section of aluminum is also plotted. 30

Figure 3.5: Vacuum chamber used for vacuum planar foil shots. 32

Figure 3.6: Ultrafast framing camera (A) with zoom imaging lens (B) positioned to image a foil load (D). A sheet of Plexiglas (C) is positioned between the load and the imaging lens to act as a sacrificial window, preventing deposition on the lens.

Figure 3.7: ICCD response as a function of wavelength.

Figure 3.8: Pre-shot image of shot E-016, showing typical foil surface characteristics. Some of the bright spots result from sharp points on the surface of the foil resulting from folds; others result from more highly reflective inclusions.

Figure 3.9: Fiber imaging setup for optical emission spectroscopy and time-resolved PMT measurements. (A) Foil load hardware (B) $10 \mathrm{~cm}$ focal length lens (C) PMMA fiber optic.

Figure 3.10: PMT signal plotted alongside measured load voltage and current. The peak of the voltage spike lines up with the start of light emission; this is a consistent feature on shots fielding the PMT.

Figure 3.11: Blackbody emission in the visible spectrum for various temperatures, scaled in proportional arbitrary units. 38 
Figure 3.12: Tungsten calibration source at $2977 \mathrm{~K}$, imaged with $600 \mathrm{~ns}$ exposure at 10x magnification on the framing camera. 39

Figure 3.13: Camera response as a function of blackbody emitter temperature for exposure times of (a) $5 \mathrm{~ns}$ (b) $10 \mathrm{~ns}$ (c) $20 \mathrm{~ns}$ and (d) $80 \mathrm{~ns}$. Camera response values below 10 are considered below the noise floor, and values above 245 are outside the linear regime, limiting the effective temperature range that can be measured for a given exposure time. 41

Figure 3.14: Support structures used for liner ablation experiments. (a) Dumbbell support allowing a $1 \mathrm{~cm}$ section of the liner to implode due to magnetic pressure (b) Solid support preventing implosion (c) Assembled liner with aluminum foil in place. The ends of the support structures are wrapped with aluminum tape to allow electrical contact to the anode and cathode; the $1.5 \mathrm{~cm}$ section of foil spans the $1 \mathrm{~cm}$ gap between the tape and makes contact on both sides. All liners are $3.4 \mathrm{~cm}$ in total length

Figure 3.15: Framing camera laser shadowgraphy system. A single 2 ns laser pulse is split into multiple collinear pulses, spaced temporally by $10 \mathrm{~ns}$, using a resonating cavity consisting of mirrors M1, M2, and M3. Beam splitter BS1 inputs 5\% of the initial 2 ns pulse into the cavity and retains $95 \%$ of each subsequent pulse in the cavity, dumping the rest of the laser energy into the beam dump. Beam splitter BS2 picks off $5 \%$ of the beam energy and sends it through the load, providing the backlight for a shadowgraph image. 44

Figure 4.1: Three dimensional model of the assembled MAIZE LTD. (1) Spark gap switch; (2) $40 \mathrm{nF}$ capacitor; (3) Iron core region (core not pictured); (4) coaxial transmission line section; (5) Radial transmission line section; (6) Load region; (7) Vacuum chamber; (8) Oil chamber; (9) High voltage insulator. 
Figure 4.2: Schematic of LTD circuit. $R_{G e n}, L_{G e n}$, and $C_{G e n}$ refer to the lumped resistance, inductance, and capacitance of the 40 parallel bricks. $R_{\text {Core }}$ refers to the parallel resistance of the iron cores due to eddy currents, which dominates core behavior provided magnetic saturation is not reached. $L_{\text {Load }}$ and $R_{\text {Load }}$ are the inductance and resistance of the load. The transmission line is represented as $\mathrm{N}$ discrete elements, each with characteristic impedance $Z_{i}$

Figure 4.3: Comparison of measured current (solid curve) and simulated current (dashed curve) for a static load. The prediction agrees nearly exactly with the measurement until around 350 ns (well after peak current), after which point charge buildup on the surface of the B-dot sensors impacts their accuracy

Figure 4.4: Results from load parameter sweep showing (a) peak current in kiloamps and (b) risetime in nanoseconds at the load. Observed parameters from a sample of MAIZE shots are indicated as follows: Planar foil [49] (green square) cylindrical liner [37, 43] (red X); static resistive load (blue circle); short circuit load (black star). The rectangular region denotes the approximate parameter space spanned by the DPWA shots; the relatively large parameter space is due to shot-to-shot variation in wire dynamics as well as in-shot inductance changes due to pinching

Figure 4.5: Comparison of switch behavior over 5 consecutive shots into a static resistive load for (a) a properly functioning switch with approximately 10 ns jitter and (b) a malfunctioning switch with closing times ranging from $10 \mathrm{~ns}$ to $300 \mathrm{~ns}$ after nominal start of current. 
Figure 4.6: MAIZE vacuum chamber top down view with load hardware and diagnostics. (1) Load hardware adapter; (2) Pinhole camera with X-ray film; (3) X-ray diode; (4) Faraday Cup (in loading position); (5) KAP x-ray spectrometer; (6) Differential B-dot current probe 59

Figure 4.7: Close up isometric view of load region for DPWA experiment. (1) Planar array conisisting of six $5.1 \mu \mathrm{m}$-diameter 304 SS wires; (2) Anode return current post; (3) Cathode; (4) Anode 59

Figure 4.8: Predicted and measured current traces from (A) shot 937 and (B) shot 938 . Laser shadowgraph timings and signals from the $\mathrm{x}$-ray diodes with filters of $3.5 \mathrm{keV}$ and $9 \mathrm{keV}$ in arbitrary units are also shown. 61

Figure 4.9: Shadowgraph images from (a) shot 937 and (b) shot 938. Timing relative to start of current is indicated on each shadowgraph. Details of the dynamics of DPWA ablations can be found in References [52, 57-59]. These data were shown in Reference [36].....

Figure 4.10: Inductance change as a function of time for (a) shot 937 and (b) shot 938 (blue curves). Signals from the $3.5 \mathrm{keV}$ (green) and $9 \mathrm{keV}$ (red) x-ray are also shown. The timings of the inductance spikes agree very well with measurements of x-ray emission.

Figure 4.11: Shadowgraph taken at approximate time of peak emission from shot 937. Effective current carrying radius calculated from the inductance change is also shown. Initial interplanar wire gap spacing was $3 \mathrm{~mm}$

Figure 4.12: Predicted and measured current traces for shot 1168, an implosion of an initially solid $400 \mathrm{~nm}$ aluminum liner.

Figure 4.13: Self-emission/shadowgraph images with effective current-carrying radius indicated in white vertical bars. The first image shows a pre-shot shadowgraph for establishing 
spatial scale. The central column of the plastic support is approximately $2 \mathrm{~mm}$ in diameter, indicated by the yellow vertical bars.

Figure 4.14: Plasma column diameter measured from 10 ns-spaced framing camera images (stars) plotted against calculated current-carrying diameter (dashed curve) and load inductance (solid curve). Agreement between experiment and prediction is observed for all shadowgraph frames except the final frame at $295 \mathrm{~ns}$. The error bars represent absolute upper and lower bounds on the plasma/vacuum interface diameter given by the plasma column at its thickest and thinnest point, respectively.

Figure 5.1: Typical voltage and current plot. The voltage spikes as the resistance of the load increases sharply through the liquid and vapor phases, then drops as the vapor transitions to partially ionized plasma. For this shot, the foil dimensions were $400 \mathrm{~nm}$ x $1.0 \mathrm{~cm}$ x $0.9 \mathrm{~cm} \ldots . .69$

Figure 5.2: Measured foil resistivity, averaged over the foil and assuming the initial dimensions of the foil (solid blue curve); resistivity calculated from enthalpy delivered to the load is also plotted (dashed black curve) until the foil reaches the vaporization temperature. After this time, the resistivity of molten aluminum at the vaporization temperature is shown (dashed red line) as a reference.

Figure 5.3: Measured foil-averaged resistivity (orange curve) plotted against enthalpy delivered to the load (blue curve). Points of phase transition are shown with dashed vertical lines; these represent the onset of melting (black), the completion of melting (blue), the onset of vaporization (green), and the completion of vaporization (red), all assuming uniform energy deposition.

Figure 5.4: Typical image sequence of 5 ns-exposure images showing self-emission striations on an $800 \mathrm{~nm} \times 0.7 \mathrm{~cm} \times 0.9 \mathrm{~cm}$ foil ablation. Imaging window is $1.25 \mathrm{~mm} \times 1.08 \mathrm{~mm}$ 
with $2 \mu \mathrm{m}$ resolution. This sequence shows the stages of striation formation, merging, and eventual formation of filamentary plasma channels. Current flows from left to right. 73

Figure 5.5: Plots of PMT signal and average foil resistivity for $400 \mathrm{~nm}$ x $1.0 \mathrm{~cm}$ x $0.9 \mathrm{~cm}$ aluminum foil ablations. The same phase transition timings shown in Figure 5.3 are included. Background gases were (a) and (b) air, (c) and (d) $\mathrm{SF}_{6}$, and (e) argon, all at atmospheric pressure.

Fig. 5.6: Time- and spatially-integrated spectral measurements of foil self-emission and electrical signals from ablation of (a, b) $400 \mathrm{~nm} \times 1.0 \mathrm{~cm} \times 0.9 \mathrm{~cm}$ foil and (c, d) an $800 \mathrm{~nm} \times 0.7$ $\mathrm{cm} \times 0.9 \mathrm{~cm}$ foil. The background gas for (a) was atmospheric air, and the background gas for (c) was atmospheric pressure argon. The upward slope of the continuum is believed to be due to blackbody emission (due to an average surface temperature that changes as a function of time). Plots (b) and (d) show the corresponding voltage and current plots with the ICCD gate width shown as green boxes. Plot (e) shows a non-local thermodynamic equilibrium (NLTE) PrismSPECT simulation (provided by Dr. Sonal Patel of Sandia National Labs) for aluminium (neutral and singly ionized) in the same wavelength range.

Figure 5.7: (a) Partial image sequence showing self-emission from a $400 \mathrm{~nm} \times 1.0 \mathrm{~cm} \times$ $0.9 \mathrm{~cm}$ foil ablating in air (b) Partial image sequence showing a foil of the same dimensions ablating in atmospheric pressure $\mathrm{SF}_{6}$. All frames are $1.25 \mathrm{~mm}$ x $1.08 \mathrm{~mm}$ with $5 \mathrm{~ns}$ exposure and $2 \mu \mathrm{m}$ spatial resolution. Current flows from left to right. 83

Figure 5.8: Self-emission sequence of a $400 \mathrm{~nm}$ x $1.0 \mathrm{~cm} \times 0.9 \mathrm{~cm}$ foil ablation in vacuum. Exposure time for each image is $150 \mathrm{~ns}$, and the imaging window is the maximum available $5 \mathrm{~mm}$ x $4.32 \mathrm{~mm}(10 \mu \mathrm{m}$ resolution) to maximize light collection. Current flows from left to right 85 
Figure 5.9: Temperature plot (in Kelvin) of an 80 ns exposure image, taken on an 800 $\mathrm{nm} \times 1.0 \mathrm{~cm} \times 0.9 \mathrm{~cm}$ aluminum foil to establish background temperature during formation of striation structures. For this exposure and magnification, the camera response floor corresponds to $2600 \mathrm{~K}$, and saturation corresponds to $4200 \mathrm{~K}$. Current travels left to right 86

Figure 5.10: Temperature surface maps (in $\mathrm{K}$ ) for a $400 \mathrm{~nm}$ x $1.0 \mathrm{~cm}$ x $0.9 \mathrm{~cm}$ foil; $\mathrm{J} \approx$ $5.0 \times 10^{7} \mathrm{~A} / \mathrm{cm}^{2}$. Image sizes are $1.25 \mathrm{~mm}$ by $1.08 \mathrm{~mm}$. Current flows from left to right........ 88

Figure 5.11: Temperature surface maps (in $\mathrm{K}$ ) for an $800 \mathrm{~nm}$ x $0.7 \mathrm{~cm}$ x $0.9 \mathrm{~cm}$ foil; $\mathrm{J} \approx$ $4.0 \times 10^{7} \mathrm{~A} / \mathrm{cm}^{2}$. Image sizes are $1.25 \mathrm{~mm}$ by $1.08 \mathrm{~mm}$. Current flows from left to right. 89

Figure 5.12: Temperature surface maps (in $\mathrm{K}$ ) for an $800 \mathrm{~nm}$ x $1.0 \mathrm{~cm}$ x $0.9 \mathrm{~cm}$ foil; $\mathrm{J} \approx$ $3.9 \times 10^{7} \mathrm{~A} / \mathrm{cm}^{2}$. Image sizes are $1.25 \mathrm{~mm}$ by $1.08 \mathrm{~mm}$. Current flows from left to right. 90

Figure 5.13: Temperature surface maps (in $\mathrm{K}$ ) for a $2.0 \mu \mathrm{m} \times 0.25 \mathrm{~cm} \times 0.9 \mathrm{~cm}$ foil; $\mathrm{J} \approx$ $6.0 \times 10^{7} \mathrm{~A} / \mathrm{cm}^{2}$. Image sizes are $1.25 \mathrm{~mm}$ by $1.08 \mathrm{~mm}$. Current flows from left to right 91

Figure 5.14: Temperature surface maps (in $\mathrm{K}$ ) for a $2.0 \mu \mathrm{m} \times 0.4 \mathrm{~cm} \times 0.9 \mathrm{~cm}$ foil; $\mathrm{J} \approx$ $3.5 \times 10^{7} \mathrm{~A} / \mathrm{cm}^{2}$. Image sizes are $1.25 \mathrm{~mm}$ by $1.08 \mathrm{~mm}$. Current flows from left to right. 92

Figure 5.15: Example temperature lineouts of shot E-14 (shown in Figure 5.11) plotted for multiple times during the image sequence. The y-axes are truncated at $4000 \mathrm{~K}$ because temperatures below this value cannot be resolved for the specified magnification and exposure time. 94

Figure 5.16: Fourier transforms of temperature lineouts of shot B-40 (400 nm x $1.0 \mathrm{~cm} \mathrm{x}$ $0.9 \mathrm{~cm}$ foil shown in Figure 5.10), plotted for multiple times during the image sequence. 95

Figure 5.17: Fourier transforms of temperature lineouts of shot E-14 (800 nm x $0.7 \mathrm{~cm} \mathrm{x}$ $0.9 \mathrm{~cm}$ foil shown in Figure 5.11), plotted for multiple times during the image sequence. 96 
Figure 5.18: Fourier transforms of temperature lineouts of shot E-24 (800 nm x $1.0 \mathrm{~cm} \mathrm{x}$ $0.9 \mathrm{~cm}$ foil shown in Figure 5.12), plotted for multiple times during the image sequence. 97

Figure 5.19: Fourier transforms of temperature lineouts of shot E-62 $(2.0 \mu \mathrm{m} \times 0.25 \mathrm{~cm} \times$ $0.9 \mathrm{~cm}$ foil shown in Figure 5.13), plotted for multiple times during the image sequence. 98

Figure 5.20: Fourier transforms of temperature lineouts of shot E-62 $(2.0 \mu \mathrm{m} \times 0.25 \mathrm{~cm} \times$ $0.9 \mathrm{~cm}$ foil shown in Figure 5.13), plotted for multiple times during the image sequence. 99

Fig 5.21: Growth rate calculations for lineouts shown in Figure 5.15. Amplitude is plotted as a function of time and fit to an exponential function; the coefficients of several of these functions are then averaged to obtain an average growth rate for the shot. 101

Fig 5.22: Instantaneous growth rates calculated from (2.18) with the imaging window indicated as a green box. The measured average growth rate is shown as a dashed horizontal line to compare with the theoretical values. The blue curve, which corresponds to $k=10^{4} \mathrm{~m}^{-1}$, is taken to represent $\gamma \max$, as the corresponding wavelength is nearly two orders of magnitude greater than $\lambda \mathrm{min}$. The average growth rate in the observation window is expected to fall between the average value within this window for $\lambda=30 \mu \mathrm{m}$ (represented by the yellow curve) and the average value of $\gamma \max$ (represented by the blue curve). 102

Figure 5.23: Experimentally measured growth rates from Table 5.1 plotted against current density. Results are consistent with quadratic dependence on current. Note that for large features with growth rate described by (5.9), the y-intercept should be approximately 0 (which has been enforced on the fit), so the data do not as strongly support a linear fit with this criterion.

Figure 5.24: (a) Pre-shot image showing deep grooves embedded in an $800 \mathrm{~nm}$ x $0.7 \mathrm{~cm}$ x $0.9 \mathrm{~cm}$ foil, to approximate resistivity jumps at grain boundaries. Grooves are approximately 
$320 \mu \mathrm{m}$ apart. (b) Temperature plot sequence showing instability growth. All image sizes are $1.25 \mathrm{~mm} \times 1.08 \mathrm{~mm}$; current flows from left to right 106

Figure 5.25: (a) Pre-shot image showing two holes with diameter $>100 \mu \mathrm{m}$ punctured in a $400 \mathrm{~nm} \times 1.0 \mathrm{~cm} \times 0.9 \mathrm{~cm}$ foil, to approximate large areas of high resistivity. The hole locations are highlighted in the yellow circles (b) Temperature plot generated from image taken at $220 \mathrm{~ns}$ showing ETI preferentially forming along the line between the holes before growing elsewhere along the foil (c) Full shot temperature sequence. All image sizes are $2.5 \mathrm{~mm}$ x 2.15 $\mathrm{mm}$; current flows from left to right. 108

Figure 5.26: Temperature surface plot of an $800 \mathrm{~nm}$ x $0.7 \mathrm{~cm}$ x $0.9 \mathrm{~cm}$ aluminum foil ablation showing plasma from vaporized sections of the foil joining to form filamentary structures late in time. 110

Figure 6.1: Typical current trace with shadowgraph/self-emission image timings indicated on the figure. The $557 \mathrm{kA}$ peak current and $240 \mathrm{~ns}$ risetime were characteristic of liner load shots.

Figure 6.2: Image sequence of a $400 \mathrm{~nm}$ bare aluminum liner showing well-developed MRT coupled to the sausage mode. These liners had the lowest linear mass of all liners fielded and exhibited the most dramatic implosion.

Figure 6.3: Image sequence of a $200 \mathrm{~nm}$ aluminum liner with a $1.5 \mu \mathrm{m}$ ultra-polyester coating on the inner surface. The higher linear mass of these liners compared to the bare aluminum resulted in lower acceleration and less MRT growth. 115

Figure 6.4: Image sequence of a $250 \mathrm{~nm}$ titanium liner with a $1.5 \mu \mathrm{m}$ ultra-polyester coating on the inner surface. This liner has the highest linear mass that has been observed to 
exhibit implosion on MAIZE, although the amount of inward motion of the plasma-vacuum interface is minimal.. 116

Figure 6.5: Interface analysis of a region of interest of an ultra-polyester backed $400 \mathrm{~nm}$ aluminum liner region of interest at $400 \mathrm{~ns}$. (a) Interface fit (b) Interface perturbation functions for the left and right plasma-vacuum interfaces on Figure 6.5a. The RMS amplitude of these perturbations calculated from (6.1) is $0.28 \mathrm{~mm}$ for the left interface and $0.19 \mathrm{~mm}$ for the right interface (c) Fourier transforms of the perturbation functions.

Figure 6.6: Fourier transforms of plasma-vacuum interfaces for aluminum and titanium liners at times from 120 to $280 \mathrm{~ns}$ after start of current. While the titanium liners show slightly more growth of long-wavelength features, overall both materials exhibit similar wavelength spectra.

Figure 6.7: Instability amplitude determined from (6.1) as a function of time for aluminum and titanium liners. The growth is similar for the two metals.

Figure 6.8: $400 \mathrm{~nm}$ aluminum liner ablation on solid plastic support; a lower frame rate was used to capture the evolution of the sausage mode instability over several hundred ns 123

Figure 6.9: $500 \mathrm{~nm}$ tantalum liner ablation on solid plastic support imaged using the same frame rate as the aluminum liner in Figure 6.8. The plasma-vacuum interfaces are much more stable than in Figure 6.8. A small sausage instability structure believed to be seeded by edge effects can be seen on the bottom left of the liner interface, demonstrating that sausage mode can grow in this system when it is seeded. Later images were contrast-enhanced to make the interface visible. 124

Figure 6.10: $500 \mathrm{~nm}$ tantalum liner ablation on dumbbell plastic support imaged at similar times as the liner in Figure 6.9a. The nearly static plasma-vacuum interface in this shot 
rules out the stabilizing effect of acceleration in the radially outward direction. Very little instability growth is observed.

Figure 6.11: Instability amplitude vs time for the shots depicted in Figures 6.8 and 6.9. While instability grows exponentially for the aluminum liner, the amplitude of instabilities on the tantalum liners remain on the order of the imaging resolution for over $400 \mathrm{~ns}$.

Figure 6.12: Shadowgraph sequence of exploding Al liner ( $400 \mathrm{~nm})$ with no coating . 129 Figure 6.13: Shadowgraph sequence of exploding Al liner (400 nm) with ultra-polyester coating on inside 130

Figure 6.14: Shadowgraph sequence of exploding Al liner (400 nm) with ultra-polyester coating on outside

Figure 6.15: Contrast enhanced liner ablation images at $330 \mathrm{~ns}$ after start of current for (a) bare aluminum (b) plastic-on-inside aluminum and (c) plastic-on-outside aluminum. The same images are reproduced in (d) through (f) to highlight the presence of dark striations. These striations are exclusively horizontal and connect azimuthally correlated instability structures for cases (a) and (b); in case (c) both horizontal and angled striations are present. 132

Figure 6.16: Fourier transforms of the interfaces for all 3 cases at various times after start of current. In general, instability structures grow more and become larger in wavelength for the bare aluminum shots (shown in blue) and plastic-on-inside shots (red) than for the plastic-onoutside case (purple).

Figure 6.17: Instability amplitude as a function of time for all 3 cases. As expected, the bare aluminum shot (plotted in blue) exhibits the highest growth rate, and the plastic-on-inside cases (red) also show growth. While shot 1196 exhibits the expected behavior for the plastic- 
on-outside case (purple), shot 1201 shows a surprisingly large, albeit slowly growing, instability amplitude.

Figure 6.18: Interface correlation function vs time for all 3 cases calculated from (6.2). As anticipated, the bare aluminum (blue) and plastic-on-inside (red) cases are highly azimuthally correlated, while the plastic-on-outside (purple) shots exhibit markedly less correlation. 136

Figure 7.1: Temperature surface plot of an $800 \mathrm{~nm} \times 0.7 \mathrm{~cm} \times 0.9 \mathrm{~cm}$ aluminum foil ablation showing plasma from vaporized sections of the foil joining to form filamentary structures late in time. 143

Figure C.2: Fully assembled switch exterior view .............................................. 166

Figure C.2: Schematic of fully assembled switch interior view .............................. 167

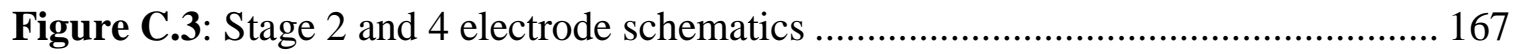

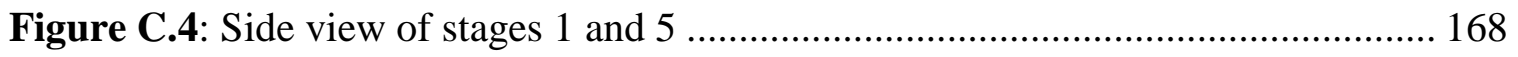

Figure C.5: Anode and cathode electrode plate schematics .................................. 168

Figure C.6: PMT signal from a switch during the breakdown process. ...................... 172

Figure C.7: PMT signals from a properly performing switch. Note the normal $10 \mathrm{~ns}$

jitter. 173

Figure C.8: PMT signals from a switch requiring rebuilding. Note the normally $10 \mathrm{~ns}$ jitter exhibited in Figure C.7 has increased to almost 200 ns. 174 


\section{LIST OF TABLES}

Table 4.1 Physical scales used as normalization constants for the MAIZE circuit model.

Table 5.1: Measured ETI growth rates for various foil masses and geometries, listed along with theoretical growth rates for long-wavelength modes (the maximum growth rate predicted by theory) and theoretical growth rates for $\lambda=30 \mu \mathrm{m}$ (a consistently observed smallest

growing feature size that appeared to be independent of foil mass). 103 


\section{LIST OF APPENDICES}

APPENDIX A LTSPICE Simulation of MAIZE with Independent Brick Trigger Timings ..... 144

APPENDIX B MATLAB Code for Simulating MAIZE and Calculating Load Inductance ...... 151

APPENDIX C Switch Description and Rebuild Procedure for MAIZE 166

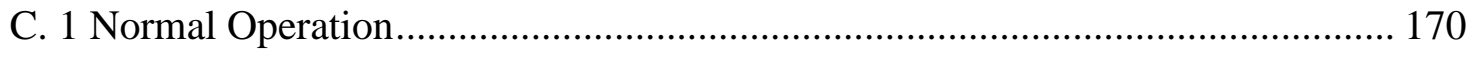

C.2 Sequence Multi-Electrode Breakdown Theory ........................................... 170

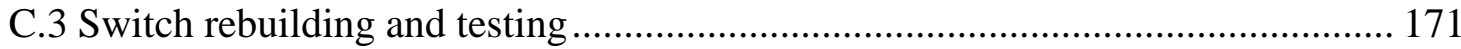




\section{ABSTRACT \\ The Electrothermal Instability on Pulsed Power Ablations of Thin Foils \\ by}

\section{Adam M. Steiner}

\section{Chair: Ronald M. Gilgenbach}

The electrothermal instability (ETI) is an exponentially growing temperature perturbation that arises due to nonuniformities in Ohmic heating of a current-carrying material with a temperature-dependent resistivity. When resistivity increases with temperature, as in most solid and liquid metals, ETI forms striations of hot and cold material perpendicular to the flow of current. On a pulsed-power driven ablation of an initially solid metal, these striations can cause local vaporization before the bulk material vaporizes, leading to a mass perturbation that can seed plasma instabilities, such as the magneto Rayleigh-Taylor (MRT) instability. These instabilities have been identified as the primary impediment to producing energy gain in a pulsed power-driven nuclear fusion concept called magnetized liner implosion fusion (MagLIF). Understanding of ETI may provide better means to mitigate plasma instabilities and achieve fusion gain on MagLIF experiments.

A diagnostic has been developed to measure spatially resolved temperature using an ultrafast framing camera from self-emission of planar foil ablations conducted in atmospheric conditions. These temperature measurements provide the first time-resolved experimental 
observations of ETI as a growing temperature perturbation on ablations of initially solid metal targets. Growth rates of experimentally observed perturbations show good agreement with theoretical predictions of ETI and demonstrate expected quadratic dependence on current density.

Additional experiments were conducted on the MAIZE linear transformer driver (LTD), a 1-MA pulsed power facility at the University of Michigan, to study the coupling of ETI to latertime plasma instabilities. Liners of aluminum, titanium, and tantalum were ablated to compare material-dependent effects, and ablations of aluminum with and without dielectric coatings (which had previously been shown to reduce the impact of ETI) were performed to compare instability growth on the same material with varying ETI seeding. It was observed that tantalum liners, which have lower predicted ETI growth, exhibit dramatically less plasma instability growth than aluminum or titanium. Additionally, ablations of aluminum liners with external dielectric coatings exhibited less azimuthal symmetry than bare aluminum liners, which was anticipated because ETI tends to azimuthally self-correlate. These results support the theory that ETI provides the surface perturbation that is responsible for seeding plasma instabilities on liner ablations. 


\section{CHAPTER 1}

\section{Introduction}

Harnessing fusion energy as a practical energy source has been a grand challenge endeavor for plasma physicists over the past several decades. Because nuclear fusion only occurs at extreme temperature regimes at which kinetic energy of like-charged nuclei is sufficient to overcome the Coulomb barrier, fusion experiments focus on confining material in the plasma state for sufficient times to allow generated energy to overcome the energy required to heat the plasma. Two prominent approaches have arisen to achieve this condition: magnetic confinement, which employs strong magnetic fields in geometries that hold the plasma in steadystate or quasi steady-state, and inertial confinement, in which massive power fluxes are delivered to fusion fuel through either lasers or energetic particle beams, heating the plasma on a timescale that is faster than material expansion. More recently, a concept that combines elements of these two approaches has been developed, called magnetized liner implosion fusion (MagLIF) [1-2]. In MagLIF, a cylindrical metallic liner encases fusion fuel, usually deuterium-tritium (DT). An axial magnetic field is applied to the liner, and the interior fuel region is preheated by depositing laser energy. A large current pulse is driven through the liner, which implodes due to the Lorentz force (it can be easily shown that the Lorentz force is always radially inward for a current-carrying cylinder), compressing and heating the fuel. Simulations [3] and experiments $[4,5]$ on MagLIF have been performed at Sandia National Labs over the past several years. Promising recent results on liner compression $[5,6]$ have motivated a proposal to construct a 
next-generation pulsed power machine [7-9] with the goal of producing energy gain from MagLIF in the laboratory setting [10]. MagLIF is also interesting basic science in its own right, combining elements of pulsed power science and engineering, plasma physics, and high energy density matter.

One of the key issues to overcome with MagLIF is the presence of hydrodynamic plasma instabilities, such as the magneto Rayleigh-Taylor (MRT) instability, $\mathrm{m}=0$ sausage mode, and $\mathrm{m}=1$ kink mode, which can form as the surface of the imploding liner ablates into plasma. These instabilities can rapidly deteriorate liner integrity during the implosion, causing fuel to escape and lose energy. As with any exponentially increasing perturbation, these instabilities require an initial seed from which to grow. Currently, the leading theory is that the electrothermal instability (ETI) provides this initial interface perturbation [11].

ETI grows in any current-carrying material that has a temperature-dependent resistivity, $\eta(T)$. If $\partial \eta / \partial T$ is negative, as in dielectrics, semiconductors, and strongly ionized plasmas, ETI tends to grow filamentary structures parallel to current direction. However, if $\partial \eta / \partial T$ is positive, as in most solid and liquid metals, ETI produces striations of hot and cold material that are perpendicular to the flow of current. These hot striations ablate before the bulk material, creating a perturbation in both mass density and interface position; this perturbation serves as a seed for plasma instabilities. The purposes of the work presented in this dissertation are to expand the understanding of ETI through experimental observation of its growth as a temperature perturbation and to identify the effects of varying the amount of ETI growth on the subsequent development of plasma instabilities.

A theoretical introduction to ETI is presented in Chapter 2. Chapter 2 also reviews the necessary material properties data to calculate theoretical ETI growth rates, which in general 
depend both on current density and temperature-dependent material properties. Experimental configurations and procedures are presented in Chapter 3. These experiments primarily include atmospheric ablations of thin aluminum foils driven by a small pulsed power generator and liner ablations performed on the MAIZE linear transformer driver (LTD). Chapter 3 also details the relevant current, voltage, and optical diagnostics fielded on these experiments, most importantly an ultrafast framing camera imaging system that allowed for temporally and spatially resolved temperature measurements of ablating foils.

Chapter 4 presents a detailed analysis of the MAIZE LTD. This machine is the primary pulsed power device used for MagLIF-relevant science in the Plasma, Pulsed Power, and Microwave Laboratory at the University of Michigan. The understanding of LTD behavior is important to this research, as the experimental studies performed on exploding and imploding liners used MAIZE as the current driver. LTD studies are also important generally to MagLIF, because the proposed Z-300 and Z-800 machines at Sandia National Labs that are under consideration to drive energy-producing MagLIF experiments consist of large arrays of LTDs [79].

The primary results observing the growth of ETI are presented in Chapter 5. These measurements represent the first time-resolved (12 frames per shot) direct experimental observation of ETI as a temperature perturbation. It is demonstrated in Chapter 5 that selfemission from ablating liners using the small pulsed power generator results from sections of the foil vaporizing and breaking down into plasma while the bulk foil is still in a biphase state at the vaporization temperature. This self-emission is shown to be blackbody dominated and used to establish an estimate of position-dependent temperature on each frame. Average growth rates of temperature perturbations above the background vaporization temperature are measured and 
compared to linear ETI theory. While the temperature perturbations are observed to be nonlinear on the timescales of observation, surprisingly good agreement is shown between the measured average growth rates and the theory. Additionally, the measured growth rates exhibit a theoretically predicted quadratic dependence on current density.

Chapter 6 details the interaction between ETI and later time plasma instabilities. Liner implosions and explosions are conducted on MAIZE, primarily to view the MRT and $\mathrm{m}=0$ sausage instabilities. Shots are conducted to compare growth rates of aluminum liners with liners of other materials, and to compare uncoated aluminum liners to aluminum liners with dielectric coatings. It is observed that changing liner material from aluminum to titanium, which has a higher theoretical instantaneous growth rate of ETI, has little effect, but changing material from aluminum to tantalum, which has a lower theoretical integral growth rate, dramatically reduces observed instabilities. The preferential seeding of azimuthally correlated $\mathrm{m}=0$ modes over higher azimuthal mode number modes is investigated in experiments with the added dielectric coating. Shots with the coating exhibit significantly less growth of azimuthally correlated instability compared to those with no coating, demonstrating that the self-correlating ETI is likely responsible for seeding the observed $\mathrm{m}=0$ modes. Finally, conclusions and suggested future work are presented in Chapter 7. 


\section{CHAPTER 2}

\section{Theory of the Electrothermal Instability}

\subsection{Qualitative Description of ETI}

ETI arises whenever current flows through a material that exhibits temperature-dependent resistivity. This instability has been studied since at least the 1960 s $[12,13]$ as a means of explaining the formation of filamentary structures along the direction of current in astrophysical

plasmas. A nonlinear form of the instability has also been identified, primarily for astrophysical applications, and referred to in the literature as the radiative-condensation instability [14, 15]. On pulsed power machines, ETI has been studied as the mechanism for filament formation on gas-puff Z-pinches [16, 17]. ETI has also been studied as an early-time effect on ablation of metallic targets [18-21] to explain the formation of striations perpendicular to current that have been observed since the 1950s on exploding wires [22], occurring on timescales faster than could be explained by MHD instabilities alone. Most recently, the striation form of ETI has been studied on rods [11, 23, 24] and imploding liners [6] at Sandia National Labs as a seed for hydrodynamic instabilities, most notably the MRT instability.

In general, ETI describes growth of a temperature perturbation caused by positive feedback from the temperature dependence of resistivity. When resistivity increases with increasing temperature, as in most solid and liquid metals, ETI leads to the growth of perpendicular striations; when resistivity decreases with increasing temperature, as in Spitzer- 
like plasmas and most dielectrics, ETI leads to the growth of parallel filamentations. A description of the qualitative mechanism driving this behavior follows.

First consider a circuit consisting of $N$ identical temperature-dependent resistors connected in series to a current source, as shown in Figure 2.1. The current through each resistor is the same as the applied current $I(t)$, and the power deposited into the $i$ th resistor is $P_{i}(t, T)=$ $I(t)^{2} R_{i}(T)$. If the resistivity, $\eta$, increases with temperature $\left(\frac{\partial \eta}{\partial T}>0\right)$, as in most metals, adding a small temperature increase $\delta T$ to the $i$ th resistor raises its resistance by a corresponding $\delta R$, increasing the deposited power. This process causes the temperature of the $i$ th resistor to grow faster than that of the other resistors, creating a positive feedback cycle that continues as long as $\frac{\partial \eta}{\partial T}$ is positive. If $\frac{\partial \eta}{\partial T}<0$, as in Spitzer-like plasmas, any temperature difference between resistors damps because less power is deposited into hotter resistors.

If instead the resistors are connected in parallel, as in Figure 2.2, the instability trends are reversed. In this case, the current $\mathrm{I}(\mathrm{t})$ divides among the $N$ resistors, with the $i$ th current determined from

$$
I_{i}(t, T)=\frac{I(t)\left(\sum_{k=1}^{N}\left(R_{k}(T)\right)^{-1}\right)^{-1}}{R_{i}(T)}=\frac{I(t) * R_{e q}(T)}{R_{i}(T)}
$$

and the power dissipated in the $i$ th resistor is

$$
P_{i}(t, T)=I_{i}^{2}(t, T) R_{i}(T)=\frac{I(t)^{2} * R_{e q}(T)^{2}}{R_{i}(T)}
$$

so the fraction of the power deposited in the $i$ th resistor is $\frac{R_{e q}(T)}{R_{i}(T)}$. If $\frac{\partial \eta}{\partial T}<0$, a small temperature increase $\delta T$ lowers the resistance of the $i$ th resistor by a corresponding $\delta R$, raising the fraction of power dissipated in the $i$ th resistor. This process creates positive feedback, amplifying the current through one of the parallel paths. The increased Ohmic heating through this resistor 
relative to the other resistors causes its resistivity to decrease faster, further amplifying the nonuniform current division. However, this process can eventually terminate for an ideal current source, as the maximum current through the perturbed resistor cannot exceed $\mathrm{I}(\mathrm{t})$ as its resistance approaches 0 . To continue to drive the instability, either $d I / d t$ must be positive, or the power source must behave as a voltage source rather than an ideal current source. As with the series case, changing the sign of $\frac{\partial \eta}{\partial T}$ uniformly damps temperature perturbations regardless of the behavior of the power source.

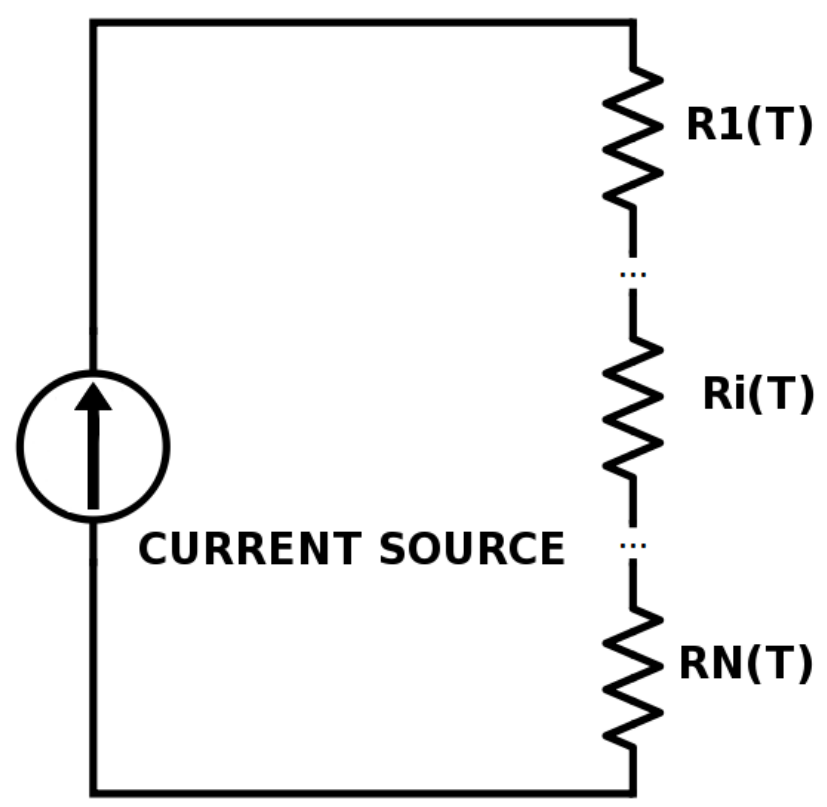

Figure 2.1: Series combination of $\mathrm{N}$ resistors with temperature dependent resistivity. A temperature perturbation applied to any resistor in the circuit causes positive feedback if $\frac{\partial \eta}{\partial T}>\mathbf{0}$ and negative feedback if $\frac{\partial \boldsymbol{\eta}}{\partial \boldsymbol{T}}<\mathbf{0}$. 


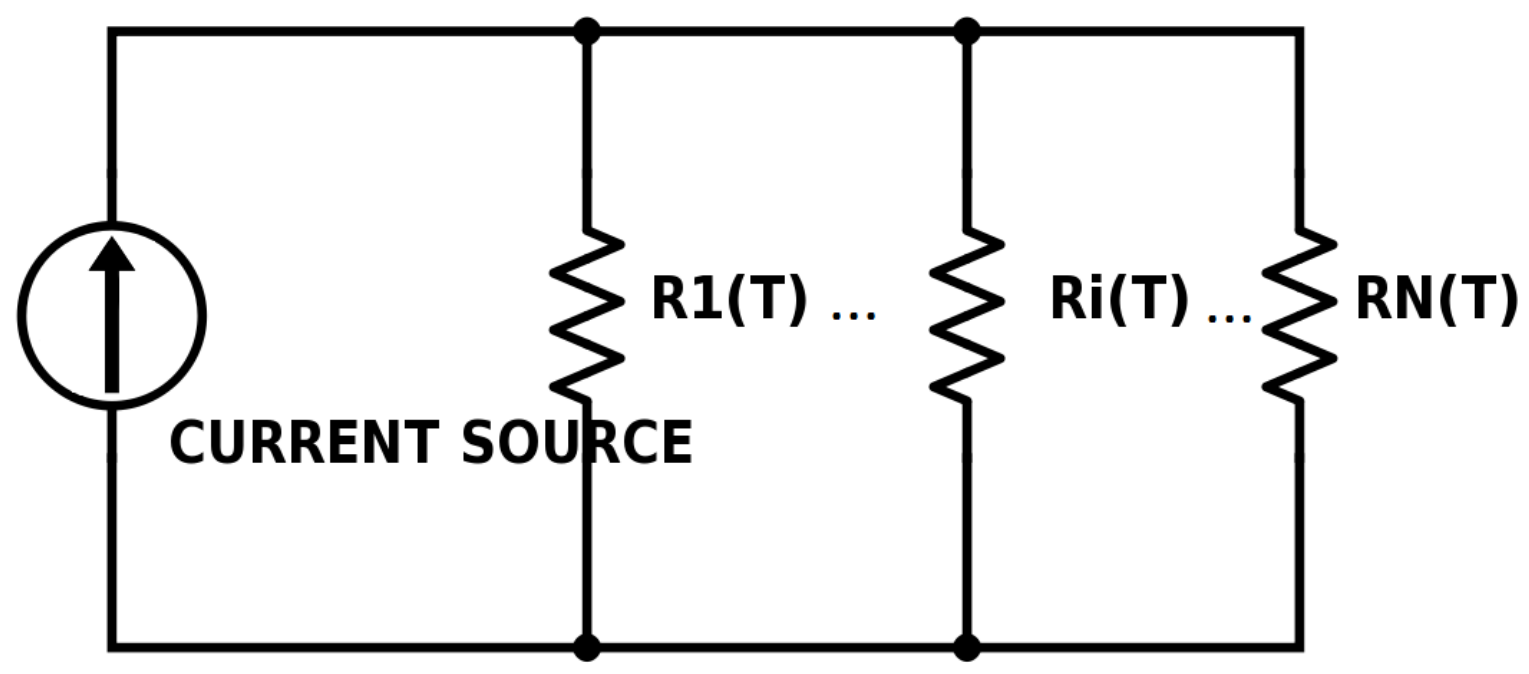

Figure 2.2: Parallel combination of $\mathrm{N}$ resistors with temperature dependent resistivity. A temperature perturbation applied to any resistor in the circuit causes positive feedback if $\frac{\partial \eta}{\partial T}<0$ and negative feedback if $\frac{\partial \eta}{\partial T}>0$.

From these simple resistor arrays, we can anticipate instability growth on a currentcarrying surface with a temperature perturbation wave vector $\bar{k}$ parallel to the direction of current, known as the striation form of ETI, when $\frac{\partial \eta}{\partial T}>0$. We also anticipate instability of a perpendicular temperature perturbation wave vector, known as the filamentation form of ETI, when $\frac{\partial \eta}{\partial T}<0$. Temperature perturbations perpendicular to the unstable direction are damped. These processes are illustrated in Figure 2.3. 


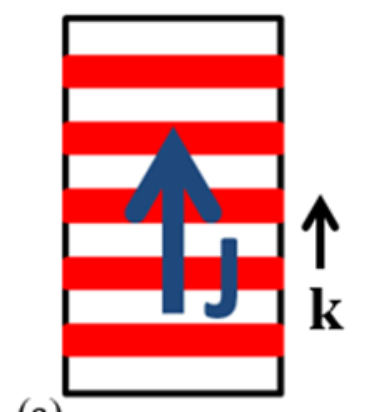

(a)

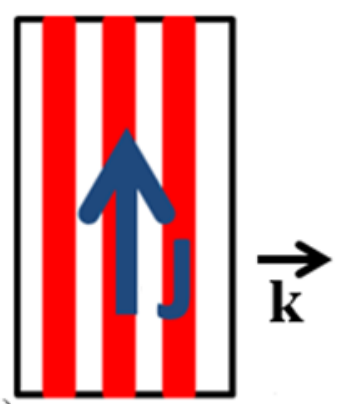

(b)

Figure 2.3: Illustration of the electrothermal instability. (a) Material with positive $\partial \eta / \partial T$ tends to grow striations of hotter, more resistive material, shown in red, with $k$ parallel to $J$. This is analogous to the series circuit described in Fig. 2.1. (b) Material with negative $\partial \eta / \partial T$ tends to grow filamentations of hotter, less resistive material, shown in red, with $k$ perpendicular to $J$. This is analogous to the parallel circuit described in Fig. 2.2.

The striation form of ETI tends to azimuthally self-correlate, as depicted in Figure 2.4a. Any initial temperature perturbations on the current carrying surface form resistive "hotspots." Current attempts to flow around these hotspots due the locally increased resistance, leading to areas of increased current density in the vicinity of the hotspots. These local areas of increased current density lead to further heating, expanding the hotspot in the perpendicular-to-current dimension. Eventually, this current spreading reaches all the way across the surface (or, equivalently, 360 degrees around a cylindrical surface), creating a fully-developed striation. Figure $2.4 \mathrm{~b}$ shows a similar process for the filamentation from. 

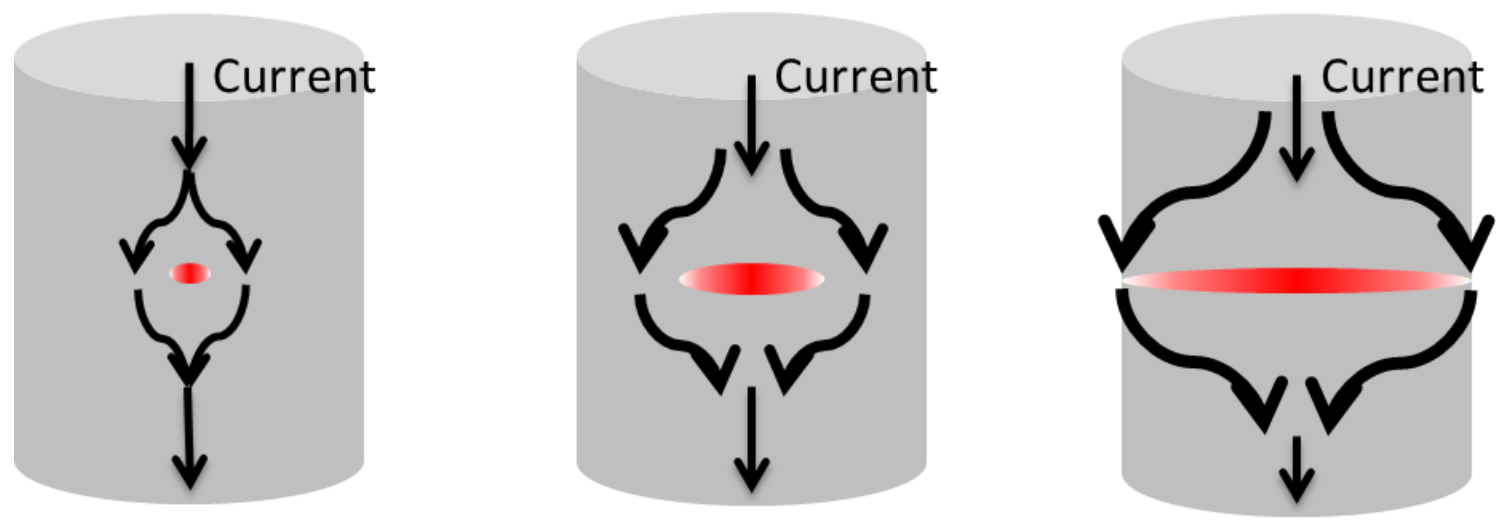

(a)
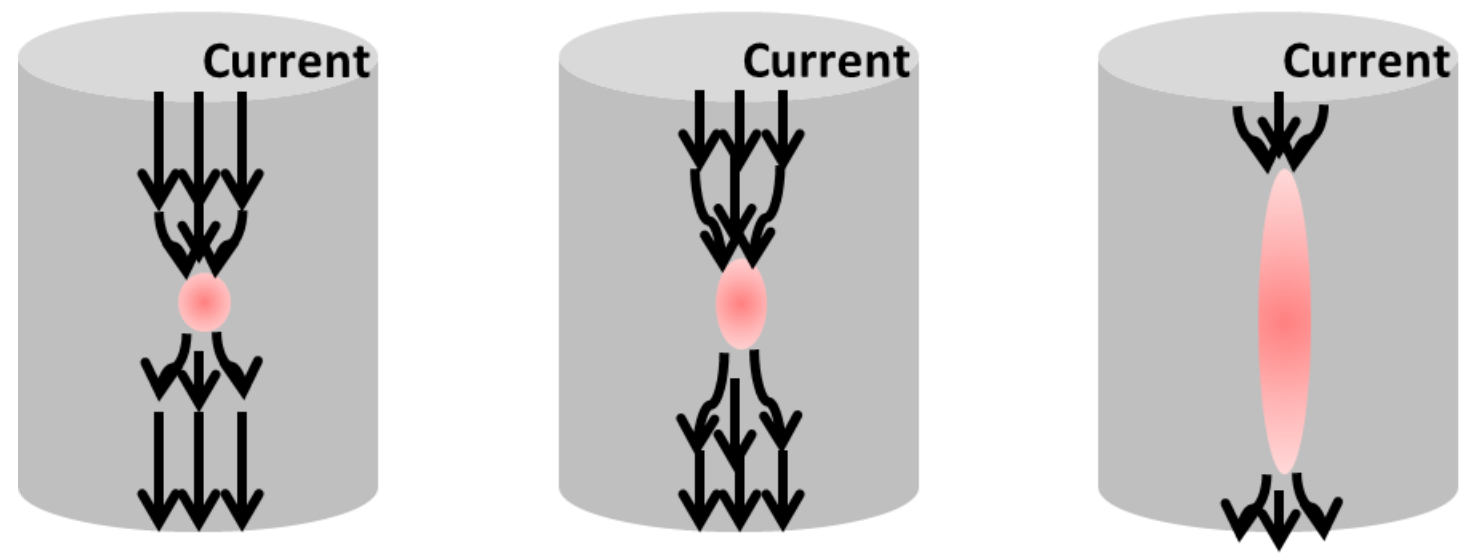

(b)

Figure 2.4: Self-correlation ETI. (a) Striation-form, current carrying material with $\partial \eta / \partial T>0$. An initial hotspot growing from nonuniformities in resistivity becomes increasingly resistive due to locally increased Ohmic heating (left). Current attempts to flow around the hotspot, bunching the current density along the edges of the hotspot, which leads to areas of increased Ohmic heating on either side of the hotspot (center). This process expands the hotspot in the direction perpendicular to current until the hotspot has become a striation (right). (b) Filamentation form, current carrying material with $\partial \eta / \partial T<0$. An initial hotspot is less resistive, drawing more current into the hotspot (left). This causes current bunching above and below the hotspot, increasing the Ohmic heating in these regions, which expands the hotspot vertically (center). This process continues in the direction parallel to current until the hotspot has become a filament. (right).

\subsection{Derivation of expansionless ETI growth rate from perturbation analysis}

The derivation of the growth rate for ETI in an expansionless, electrically thin, conducting medium has been presented by Ryutov [25] for a system in thermal equilibrium where Ohmic heating is balanced by radiative losses and by Peterson [11] for a system with time-dependent temperature and negligible radiative losses. The geometry of the problem under 
consideration is shown in Figure 2.5. Starting with Ohm's Law and Laplace's equation for magnetic field in a source free region, perturbations of the form $\exp \left(\gamma t+i k_{y} y+i k_{z} z\right)$ are considered, where $\gamma$ is the instantaneous instability growth rate (such that a positive real gamma corresponds to exponential growth) and $k_{y}$ and $k_{z}$ are the wavenumbers for perturbations in the $y$ and $z$ - directions. The foil is assumed to be electrically thin with uniform current density, which requires $d<<\delta$, where $d$ is the foil thickness and $\delta$ is the skin depth given by

$$
\delta=\sqrt{\frac{2 \eta}{\omega \mu}} \sqrt{\sqrt{1+(\eta \omega \epsilon)^{2}}+\eta \omega \epsilon}
$$

where $\eta$ is the resistivity of the foil, $\omega$ is frequency, and $\mu$ and $\varepsilon$ are the permeability and (real) permittivity, respectively. In the good conductor limit, $\eta \omega \epsilon \ll 1$, which is automatically satisfied for metallic foils with any realistic pulsed power risetime, (2.3) reduces to

$$
\delta=\sqrt{\frac{2 \eta}{\omega \mu_{0}}} .
$$

For a 100-ns risetime pulse through room temperature aluminum, this skin depth is 52 $\mu \mathrm{m}$, so the electrically thin assumption is good for foils with thicknesses on the micron and submicron scale. 


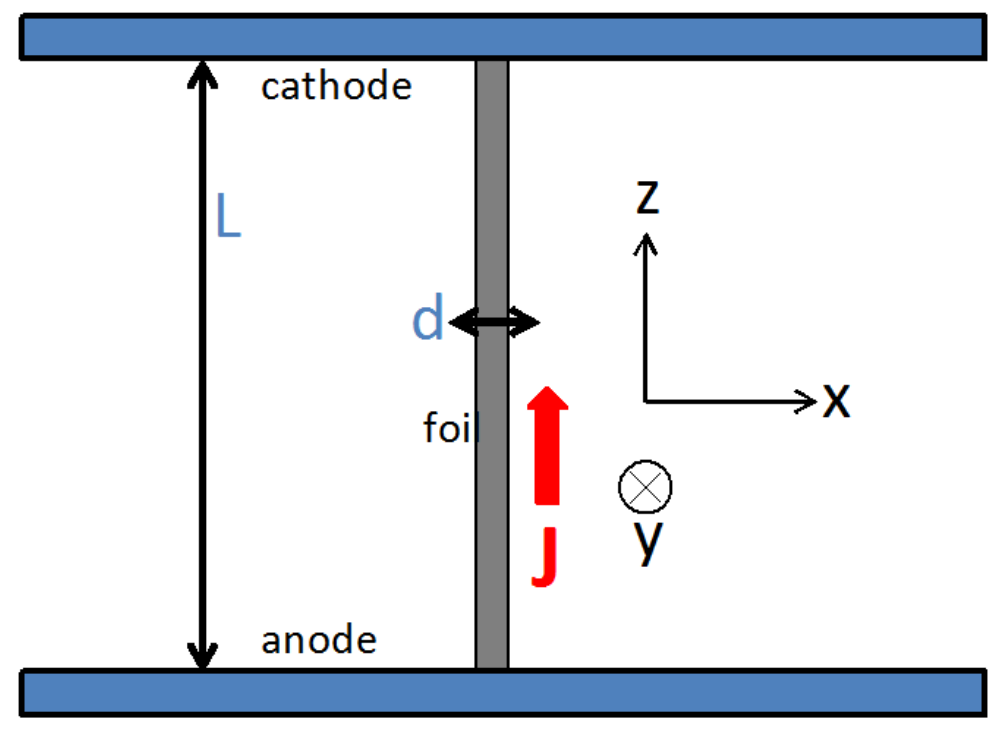

Figure 2.5: Problem geometry for ETI growth in a planar foil. Foil thickness $d$ is along the $x$-direction and current travels along the $z$-direction with AK gap spacing $L$. The foil is considered electrically thin $(d<<\delta)$.

The current density $\boldsymbol{J}$ is related to the electric field $\boldsymbol{E}$ by

$$
\boldsymbol{J}=\boldsymbol{E} / \eta
$$

A perturbation in current density is then given by

$$
\boldsymbol{\delta} \boldsymbol{J}=\frac{\boldsymbol{\delta} \boldsymbol{E}}{\eta}-\left(\frac{\delta \eta}{\eta^{2}}\right) \boldsymbol{E}
$$

Applying Ampere's and Faraday's laws to (2.6) gives the following expressions for $\delta J_{z}$ and $\delta J_{y}$ :

$$
\begin{gathered}
\delta J_{z}=-J_{z} \frac{k_{y}^{2}}{k^{2}} \frac{1}{1+\left(\frac{\gamma}{\gamma_{0}}\right)} \frac{\delta \eta}{\eta} \\
\delta J_{y}=-\left(\frac{k_{z}}{k_{y}}\right) \delta J_{z}
\end{gathered}
$$

where $k=\sqrt{k_{y}^{2}+k_{z}^{2}}$ and $\gamma_{0}$ is a characteristic growth rate given by

$$
\gamma_{0}=\frac{2 k \eta}{\mu d}
$$

Equation (2.7) can also be written as 


$$
\delta J_{z}=-J_{z} \frac{\cos ^{2} \alpha}{1+\left(\frac{\gamma}{\gamma_{0}}\right)} \frac{\delta \eta}{\eta}
$$

where $\alpha$ is the angle between an individual perturbation and the direction of current; i.e. $\alpha=0$ if $k=k_{y}$ and $\alpha=\pi / 2$ if $k=k_{z}$.

The heat equation is given by

$$
\dot{q}(t)=\frac{\partial}{\partial t}\left(T \rho(T) c_{v}(T)\right)=\nabla \cdot(\kappa(T) \nabla T)+\eta(T) J^{2}-q_{r}(T)
$$

where $\dot{q}(t)$ is the rate of change of enthalpy, $\rho(T), c_{v}(T) \kappa(T)$, and $\eta(T)$ are temperature dependent density, specific heat capacity, thermal conductivity, and electrical resistivity, respectively, and $q_{r}(T)$ is the energy lost through radiation. The terms on the right side represent (in order) thermal conduction, Ohmic heating, and radiative losses. In the case of pulsed-power driven foil ablation, radiative losses are negligible in the relatively low-temperature solid and liquid phases, and many large-scale pulsed power drivers supply sufficient Ohmic heating such that radiative losses can still be ignored well into the hot plasma regime. A perturbation $\delta T$ is introduced to perturb a quasi-equilibrium given by (2.11), where $\delta T$ is assumed to be small enough such that $\rho(T), c_{v}(T), \kappa(T)$, and $\eta(T)$ can be assumed roughly constant over the interval $[T-\delta T, T+\delta T]$, such that the values $\rho, c_{v}, \kappa$, and $\eta$ represent the density, specific heat capacity, thermal conductivity, and resistivity of the unperturbed foil at a given time:

$$
\rho c_{v} \frac{\partial(T+\delta T)}{\partial t}=\kappa \nabla^{2}(T+\delta T)+(\eta+\delta \eta)\left(J_{z}+\delta J_{z}\right)^{2} .
$$

Linearizing (2.12), neglecting second order and higher terms, and subtracting the equilibrium quantities, we obtain

$$
\rho c_{v} \gamma \delta T=-\kappa k^{2} \delta T+J_{z}^{2} \delta \eta+2 \eta J_{z} \delta J_{z}
$$

Using (2.10) along with $\delta \eta=\frac{\partial \eta}{\partial T} \delta T$ in (2.13), 


$$
\rho c_{v} \gamma \delta T=-\kappa k^{2} \delta T+J_{z}^{2} \frac{\partial \eta}{\partial T} \delta T-J_{z}^{2} \frac{\cos ^{2} \alpha}{1+\left(\frac{\gamma}{\gamma_{0}}\right)} \frac{\partial \eta}{\partial T} \delta T
$$

Rearranging gives the dispersion relation for the growth rate $\gamma$ :

$$
\gamma=\frac{J_{Z}^{2} \frac{\partial \eta}{\partial T}\left(1-\frac{\cos ^{2} \alpha}{1+\left(\frac{\gamma}{\gamma_{0}}\right)}\right)-k^{2} \kappa}{\rho c_{v}}
$$

In (2.14), the first term represents the growth of perpendicular striations of ETI, the second term represents the growth of perturbations parallel to current (and at intermediate angles to current), and the third term represents damping due to thermal conductivity.

\subsection{Characteristics of ETI growth}

The solution to (2.14) is given by

$$
\gamma=b \pm \sqrt{b^{2}-C}
$$

where $b$ and $C$ are

$$
\begin{gathered}
b=\frac{1}{2}\left(\frac{\partial \eta}{\partial T} \frac{J^{2}}{\rho c_{v}}-\frac{k^{2} \kappa}{\rho c_{v}}-\gamma_{0}\right)=\frac{1}{2}\left(\gamma_{m}-\gamma_{0}\right) \\
C=\frac{k^{2} \kappa \gamma_{0}}{\rho c_{v}}+\frac{\partial \eta}{\partial T} \frac{J^{2} \gamma_{0}}{\rho c_{v}}\left(2 \cos ^{2}(\alpha)-1\right)
\end{gathered}
$$

In (2.16) we have defined

$$
\gamma_{m}=\frac{\partial \eta}{\partial T} \frac{J^{2}}{\rho c_{v}}-\frac{k^{2} \kappa}{\rho c_{v}}
$$

It is often elucidating to rewrite the positive root of (2.15) as

$$
\gamma=\frac{1}{2}\left(\gamma_{m}-\gamma_{0}\right)+\sqrt{\left(\frac{1}{2}\left(\gamma_{m}+\gamma_{0}\right)\right)^{2}-\frac{2 \gamma_{0} J_{z}^{2} \cos ^{2} \alpha}{\rho c_{v}} \frac{\partial \eta}{\partial T}}
$$

Examining (2.15), an exponentially growing solution always exists for perturbations if $b>0$ or if $C<0$ (where we have taken the positive root in (2.15) to describe the fastest growing mode). In practice, the first condition is seldom satisfied, as a value of $\gamma_{m}$ on the order of $\gamma_{0}$ 
requires either unrealistic current densities or very large perturbation wavelengths. The second condition gives

$$
\frac{\partial \eta}{\partial T} \frac{J^{2}}{\rho c_{v}}>\frac{k^{2} \kappa}{\rho c_{v}}+\frac{\partial \eta}{\partial T} \frac{J^{2}}{\rho c_{v}}\left(2 \cos ^{2} \alpha\right)
$$

or, equivalently,

$$
\cos ^{2} \alpha<\frac{1}{2}\left(1-\frac{k^{2} \kappa}{J^{2}}\left(\frac{\partial \eta}{\partial T}\right)^{-1}\right)
$$

\subsection{1 $\partial \eta / \partial T>0$ Case}

When $\frac{\partial \eta}{\partial T}>0$ the maximum growth rate occurs when perturbations are perpendicular to the flow of current, i.e. $\alpha=\pi / 2$. In this case, the growth rate (2.19) reduces to (2.18), and the condition for instability is

$$
k<\sqrt{\frac{J^{2}}{\kappa} \frac{\partial \eta}{\partial T}}
$$

which corresponds to a minimum growing wavelength $\lambda_{\min }$,

$$
\lambda_{\text {min }}=\frac{2 \pi}{J} \sqrt{\kappa\left(\frac{\partial \eta}{\partial T}\right)^{-1}} .
$$

For wavelengths significantly larger than $\lambda_{m i n}$, the growth rate asymptotically approaches a theoretical maximum given by

$$
\gamma_{\max }=\frac{\partial \eta}{\partial T} \frac{J^{2}}{\rho c_{v}}
$$

In practice, growth rates reach $90 \%$ of this value around $3 \lambda_{\min }[19]$. For angles other than $\pi / 2$, (2.21) specifies the condition for instability, and the growth rate is found from the general relationship (2.19). For wavelengths that are large compared to $\lambda_{\min }$, the maximum growing angle $\alpha$ asymptotically approaches $\pi / 4$. 


\subsection{2 $\partial \eta / \partial T<0$ Case}

When $\frac{\partial \eta}{\partial T}>0$ the maximum growth rate occurs when perturbations are parallel to the flow of current, i.e. $\alpha=0$. In this case, the growth rate (2.19) becomes

$$
\gamma=\frac{1}{2}\left(\gamma_{m}-\gamma_{0}\right)+\sqrt{\left(\frac{1}{2}\left(\gamma_{m}+\gamma_{0}\right)\right)^{2}-\frac{2 \gamma_{0} J_{z}^{2}}{\rho c_{v}} \frac{\partial \eta}{\partial T}}
$$

In the limit $\gamma_{0} \gg \gamma_{m}, b^{2} \gg C$, allowing us to rewrite (2.15) as

$$
\gamma=|b|\left(-1+\sqrt{1-\frac{C}{B^{2}}}\right)=|b|(-1+\sqrt{1+\chi})
$$

where we have chosen the signs for the fastest growing mode and $b$ is negative definite for negative $\frac{\partial \eta}{\partial T}$. Taylor expanding about $\chi=0$,

$$
\begin{gathered}
\gamma=|b|(-1+\sqrt{1-\chi}) \approx|b|\left(-1+1-\frac{\chi}{2}\right)=\frac{-C}{|B|} \\
\gamma=\frac{\gamma_{0} \gamma_{m}^{\prime}}{\gamma_{0}+\gamma_{m}}
\end{gathered}
$$

where we have defined

$$
\gamma_{m}^{\prime}=-\frac{\partial \eta}{\partial T} \frac{J^{2}}{\rho c_{v}}-\frac{k^{2} \kappa}{\rho c_{v}}
$$

Again invoking the assumption $\gamma_{0} \gg \gamma_{m}$,

$$
\gamma=\gamma_{m}^{\prime}=\frac{J^{2}}{\rho c_{v}}\left|\frac{\partial \eta}{\partial T}\right|-\frac{k^{2} \kappa}{\rho c_{v}}
$$

Note that this growth rate is the same as (2.16) for $\frac{\partial \eta}{\partial T}>0$ striations. Using the condition $C<0$, we find a similar criterion to (2.21), but with a maximum angle condition as opposed to a minimum due to the opposite sign of $\frac{\partial \eta}{\partial T}$ :

$$
\cos ^{2} \alpha>\frac{1}{2}\left(1-\frac{k^{2} \kappa}{J^{2}}\left(\frac{\partial \eta}{\partial T}\right)^{-1}\right)
$$


Again, the critical angle in the limit $J^{2} \frac{\partial \eta}{\partial T} \gg k^{2} \kappa$ is $\pi / 4$.

\subsection{3 $\partial \eta / \partial T=0$ Case}

When the resistivity has no temperature dependence, no ETI is expected. Equation (2.19) gives

$$
\gamma=\gamma_{m}=-\frac{k^{2} \kappa}{\rho c_{v}}
$$

which simply describes thermal diffusion in the absence of driving terms.

\subsubsection{Phase Changes}

During a phase change, ETI can continue to grow, since resistivity is a function of deposited energy in a phase change. Equation (2.13) becomes

$$
\rho \gamma \delta H=-\kappa k^{2} \delta T+J_{z}^{2} \frac{\partial \eta}{\partial H} \delta H-J_{z}^{2} \frac{\cos ^{2} \alpha}{1+\left(\frac{\gamma}{\gamma_{0}}\right)} \frac{\partial \eta}{\partial H} \delta H
$$

where $\rho$ is now expressed as a function of foil enthalpy $H$ rather than $T$ (where we have chosen $\mathrm{H}$ $=0$ to represent the state at room temperature and atmospheric pressure). Provided $\delta H$ is

sufficiently small, $\frac{\partial H}{\partial T}$ is 0 during the phase transition, and the thermal conduction term drops out, giving a growth rate of

$$
\gamma=\frac{J_{Z}^{2} \frac{\partial \eta}{\partial H}\left(1-\frac{\cos ^{2} \alpha}{1+\left(\frac{\gamma}{\gamma_{0}}\right)}\right)}{\rho}
$$

where the fastest growing mode is

$$
\gamma_{m}=\frac{J_{z}^{2}}{\rho} \frac{\partial \eta}{\partial H}
$$

\subsection{Calculated Growth Rates and Instability Conditions}

Due to the tendency of the striation form of ETI to self-correlate in a direction perpendicular to current, the growth rate of the striation form of ETI on solid and liquid metals can be estimated from (2.18). On a pulsed power system, the growth rate is time-dependent for a 
given $k$ because current density and temperature are functions of time. Calculating instantaneous growth rate requires temperature-dependent values of resistivity, specific heat capacity, density, and thermal conductivity. For aluminum foils, which were used for the bulk of the experimental work described in Chapter 5 and Chapter 6, these values are determined as follows and presented in Figures 2.6 through 2.9.

Specific heat as a function of temperature was obtained from [26] as well as tabulated values from Buyco and Davis [27]. The empirical relationships [26]

$$
\begin{gathered}
C_{p}\left[\frac{k J}{k g * K}\right]=1.050\left\{H[M J / k g]<H_{s m}\right\} \\
C_{p}(T)\left[\frac{k J}{k g * K}\right]=1.070+2.308 * 10^{-5} T\left\{T_{\text {melt }}<T[K]<T_{\text {vapor }}\right\} \\
C_{p}(H)\left[\frac{k J}{k g * K}\right]=\operatorname{sqrt}\left(1.141+9.234 * 10^{-2} H\right)\left\{H_{l m}<H[M J / k g]<H_{l v}\right\}(2.36 \mathrm{c})
\end{gathered}
$$

fit these data and were used to calculate specific heat as a function of energy delivered to the load. In (2.36), $H_{s m}$ refers to the enthalpy required to heat the foil to the melting temperature, $H_{l m}$ refers to the enthalpy required to melt the foil, and $H_{l v}$ refers the enthalpy required to heat the foil to the vapor temperature, referenced to the foil at room temperature. For aluminum at atmospheric pressure, these values are $0.66 \mathrm{MJ} / \mathrm{kg}, 1.07 \mathrm{MJ} / \mathrm{kg}$, and $3.0 \mathrm{MJ} / \mathrm{kg}$, respectively.

Density was assumed to be constant in the solid phase; in the liquid phase density was calculated from tabulated values of thermal expansion coefficient [26]. During the phase change from solid to liquid, density was assumed to decrease linearly from solid density at the melting temperature to liquid density at the melting temperature. The empirical relation describing density is

$$
\begin{gathered}
\rho\left[\frac{\mathrm{kg}}{\mathrm{m}^{3}}\right]=2700\left\{H[\mathrm{MJ} / \mathrm{kg}]<H_{s m}\right\} \\
\rho\left[\frac{\mathrm{kg}}{\mathrm{m}^{3}}\right]=3150-680 H\left\{H_{s m}<H[\mathrm{MJ} / \mathrm{kg}]<H_{l m}\right\}
\end{gathered}
$$


$\rho\left[\frac{\mathrm{kg}}{\mathrm{m}^{3}}\right]=2700\left(1.021+8.378 * 10^{-2} H+4.095 * 10^{-3} H^{2}\right)^{-1}\left\{H_{l m}<H[M J / k g]<H_{l v}\right\}$

Because measurements from Reference [26] were taken at high pressures compared to the atmospheric pressure explosions addressed in this work, these values of aluminum density assume incompressibility of liquid aluminum.

Resistivity as a function of temperature was obtained from References [26] and [28]. The approximate empirical relation [26]

$$
\begin{array}{r}
\eta(H)[\mu \Omega * m]=2.65 * 10^{-2}+0.1154 H\left\{H[M J / k g]<H_{s m}\right\} \\
\eta(H)[\mu \Omega * m]=-0.0987+0.310 H\left\{H_{s m}<H[M J / k g]<H_{l m}\right\} \\
\eta(H)[\mu \Omega * m]=0.159+8.59 * 10^{-2} H+8.54 * 10^{-3} H^{2}\left\{H_{l m}<H[M J / k g]<H_{l v}\right\}
\end{array}
$$

provides unperturbed foil resistivity as a function of energy delivered to the load. Equation (2.38c) gives resistivity using temperature-dependent dimensions of aluminum as a function of input enthalpy; if the room-temperature dimensions are used in calculations (as might be done to examine a fixed area on the surface of the foil as a function of time), a modified resistivity relationship should be used in place of (2.36c) [26]:

$\eta(H)[\mu \Omega * m]=0.149+7.95 * 10^{-2} H-1.32 * 10^{-3} H^{2}\left\{H_{l m}<H[M J / k g]<H_{l v}\right\} \quad\left(2.38 \mathrm{c}^{*}\right)$ This modified resistivity function is plotted in Figure 2.10.

Thermal conductivity values were obtained from References [29] and [30]. Outside of the temperature range of the available tabulated data, thermal conductivity was calculated from the Weideman-Franz law [31]:

$$
\kappa(T)=\frac{\pi^{2} k_{B}^{2}}{3 e^{2} \eta(T)} T
$$

where $k_{B}$ is Boltzmann's constant. 

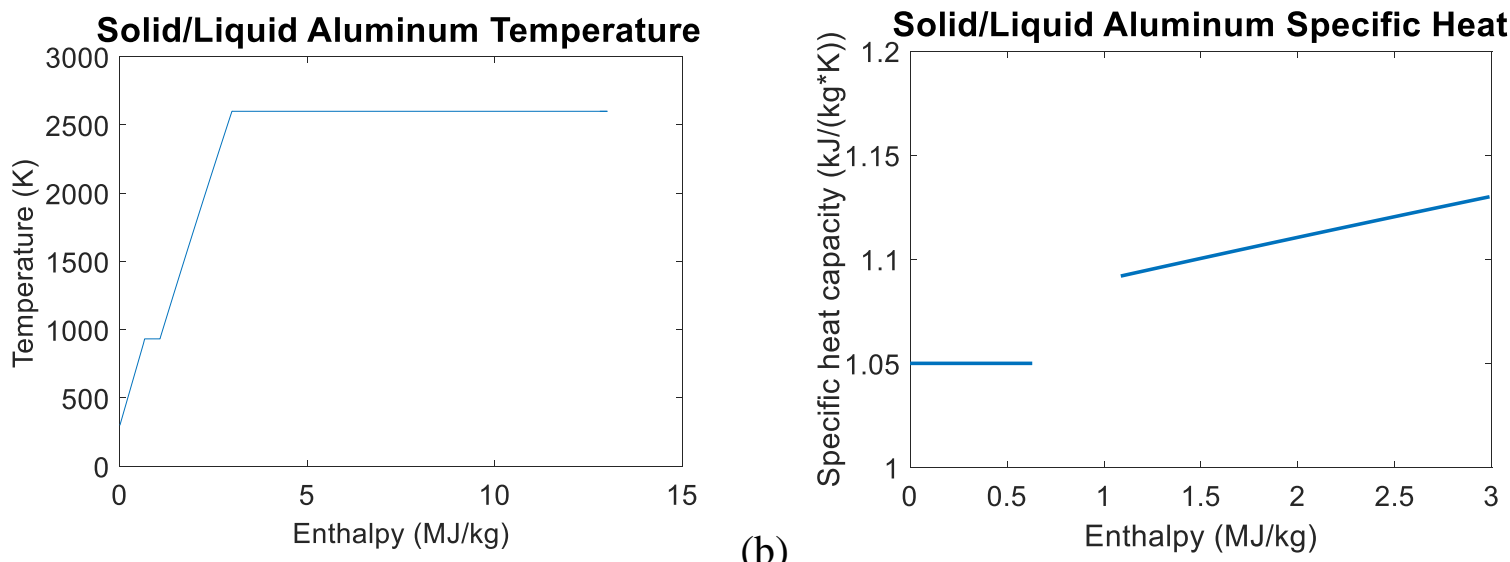

(a)

(b)

Figure 2.6: (a) Temperature of aluminum at atmospheric pressure as a function of input enthalpy $\Delta H$, where the zero of input enthalpy has been defined at room temperature, plotted up to complete vaporization at $13 \mathrm{MJ} / \mathrm{kg}$. (b)

Specific heat capacity as a function of temperature. Since the foil spends very little time in the solid phase, the solid heat capacity has been approximated as constant at the average value in the interval ( $300 \mathrm{~K}, 933 \mathrm{~K})$. Note that the heat capacity is not defined during a phase change. Empirical fits are from Reference [26].
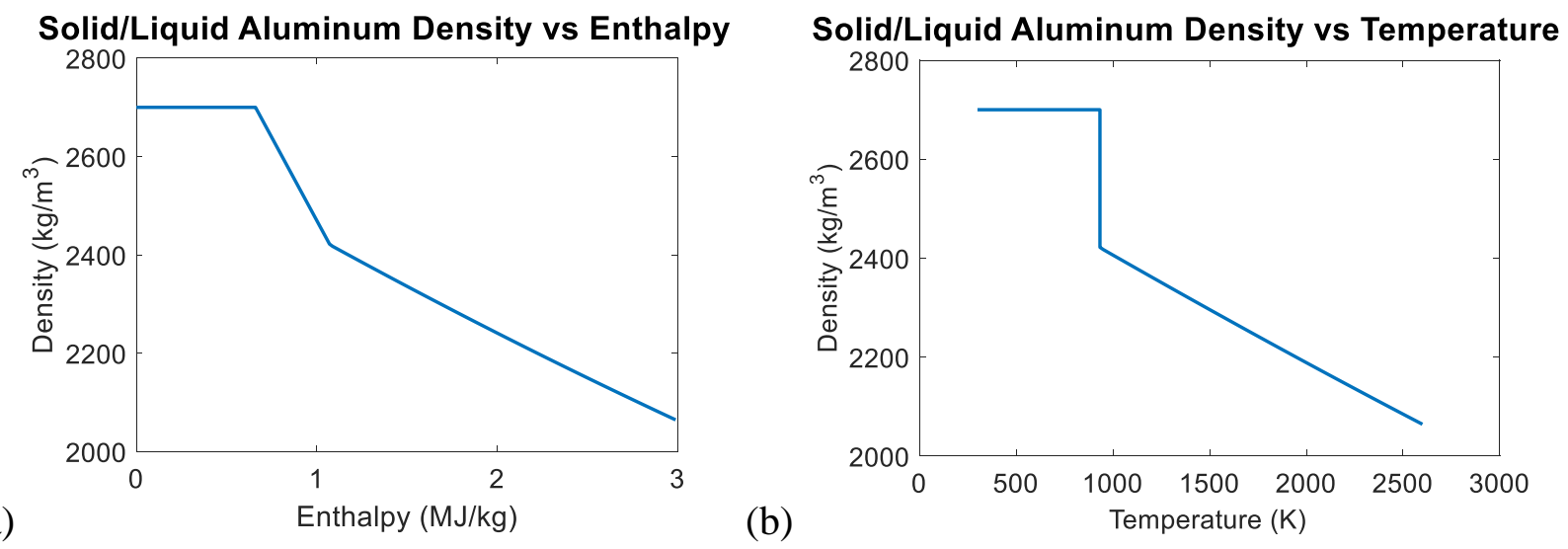

Figure 2.7: Empirical fit of solid and liquid aluminum density at atmospheric pressure (a) as a function of enthalpy and (b) as a function of temperature. Empirical fits are from Reference [26]. 


\section{Solid/Liquid Aluminum Resistivity vs Enthalpy}

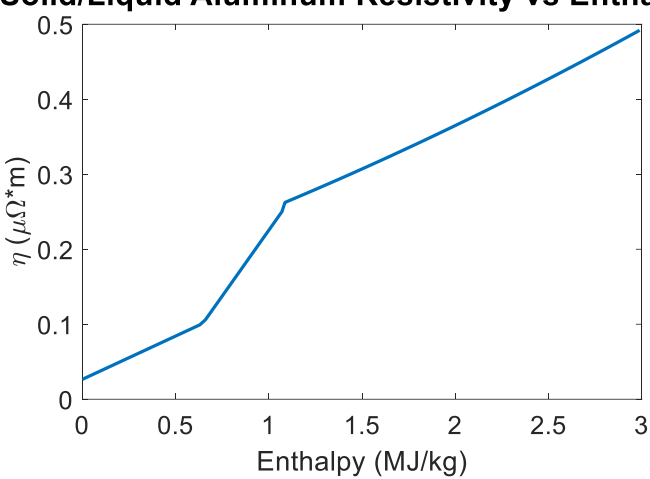

Solid/Liquid Aluminum Resistivity vs Temperature

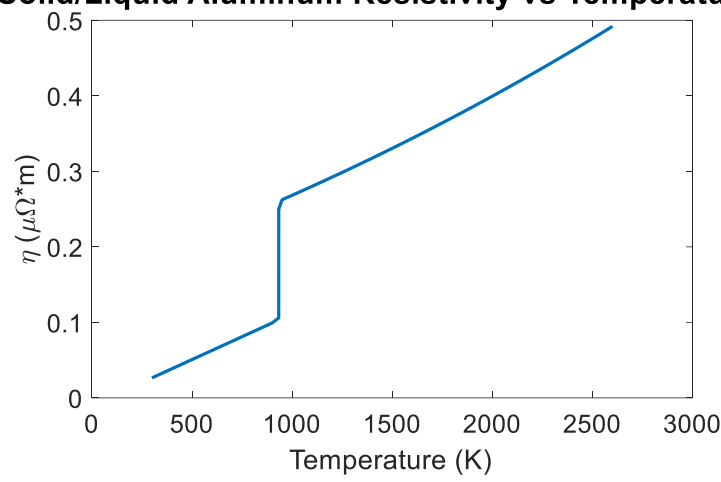

(a)

Figure 2.8: Empirical fit of solid and liquid aluminum resistivity (a) as a function of enthalpy and (b) as a function of temperature. Empirical fits are from Reference [26].

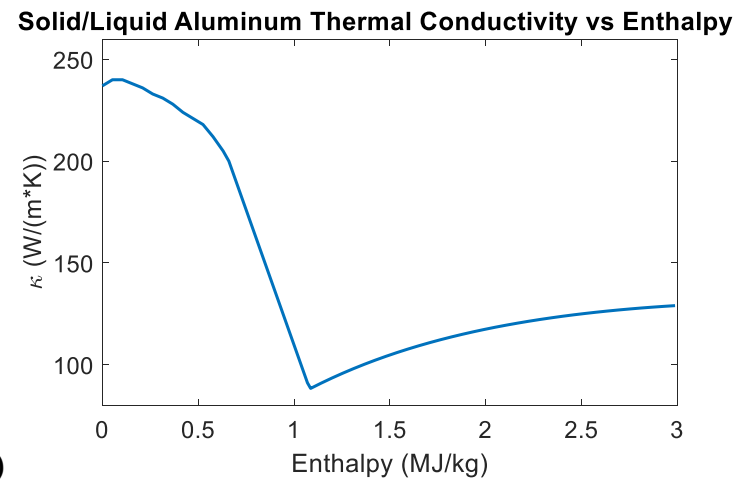

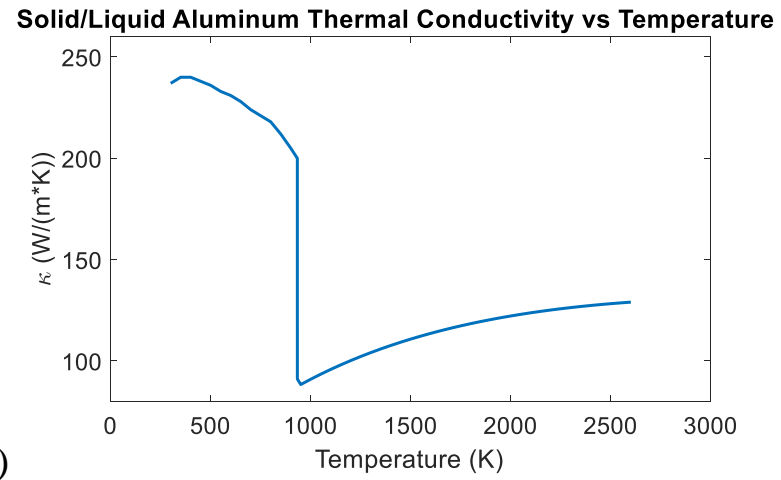

Figure 2.9: Solid and liquid aluminum thermal conductivity (a) as a function of enthalpy and (b) as a function of temperature from References [29] and [30]. At temperatures greater than $1000 \mathrm{~K}$, thermal conductivity was calculated from resistivity using the Weideman-Franz Law [31]. 

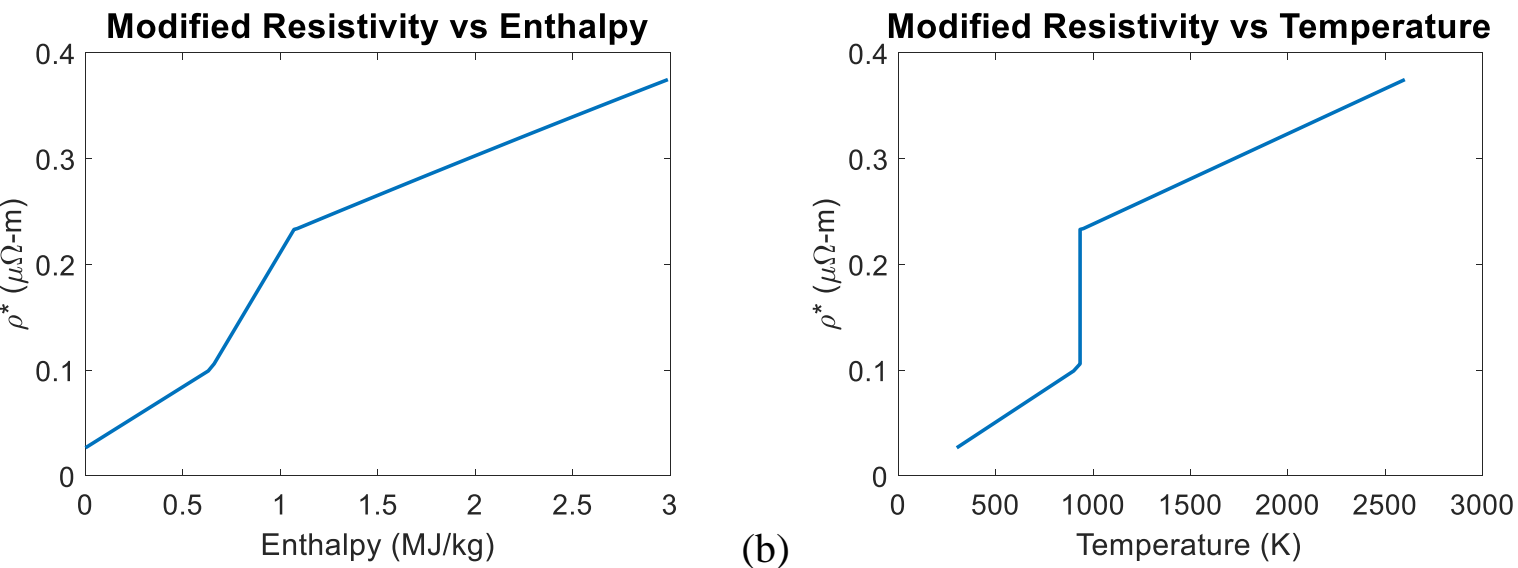

Figure 2.10: Empirical fit of solid and liquid aluminum effective resistivity of a fixed foil section, accounting for thermal expansion, plotted against (a) enthalpy and (b) temperature. Empirical fits are from Reference [26].

Figure 2.11 shows a sample striation-form $\left(k=k_{z}\right.$, where $z$ is the direction of current) ETI growth rate calculation for a $4 \mathrm{kA}, 600 \mathrm{~ns}$ risetime current pulse into an aluminum foil of $800 \mathrm{~nm}$ thickness, $7 \mathrm{~mm}$ width, and $9 \mathrm{~mm}$ length. Voltage across and current through the foil were monitored, allowing for measurements of deposited energy and current density. These measurements were used, along with the relationships (2.36) through (2.39), to calculate ETI growth rate as a function of time for various values of $k$. 


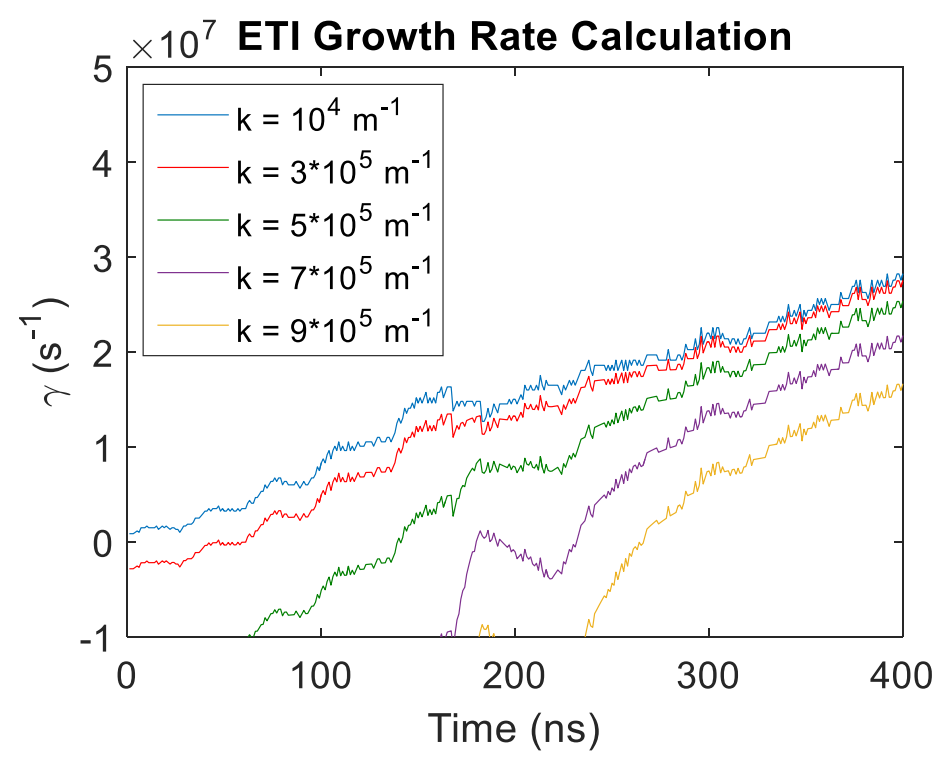

Figure 2.11: Growth rates for various values of $k$, calculated from equation (2.18) for a 4 kA, 600 ns risetime pulse into a $800 \mathrm{~nm} \times 9.0 \mathrm{~mm}$ x $7.0 \mathrm{~mm}$ aluminum foil. Experimentally measured current and voltage were used to calculate energy delivered to the foil, which was used with equations (2.36) through (2.39) to calculate current density, resistivity, specific heat, density, and thermal conductivity as a function of time for the bulk (unperturbed) foil.

Minimum growing ETI wavelength and minimum supported angle $\alpha$ are shown in Figure 2.12. From Figure 2.12a, it is apparent that minimum growing wavelength changes dramatically over the course of a foil explosion, and in general only long wavelengths can grow early in time. This may explain the counterintuitive observation [11] that varying surface roughness has little effect on the growth of ETI: typical surface roughness values are below the minimum growing wavelength early in time, so temperature perturbations on this scale exponentially decay. Figure $2.12 \mathrm{~b}$ shows that the maximum supported wavelength asymptotically approaches the predicted value of $\pi / 4$ for large current density or small $k$. 

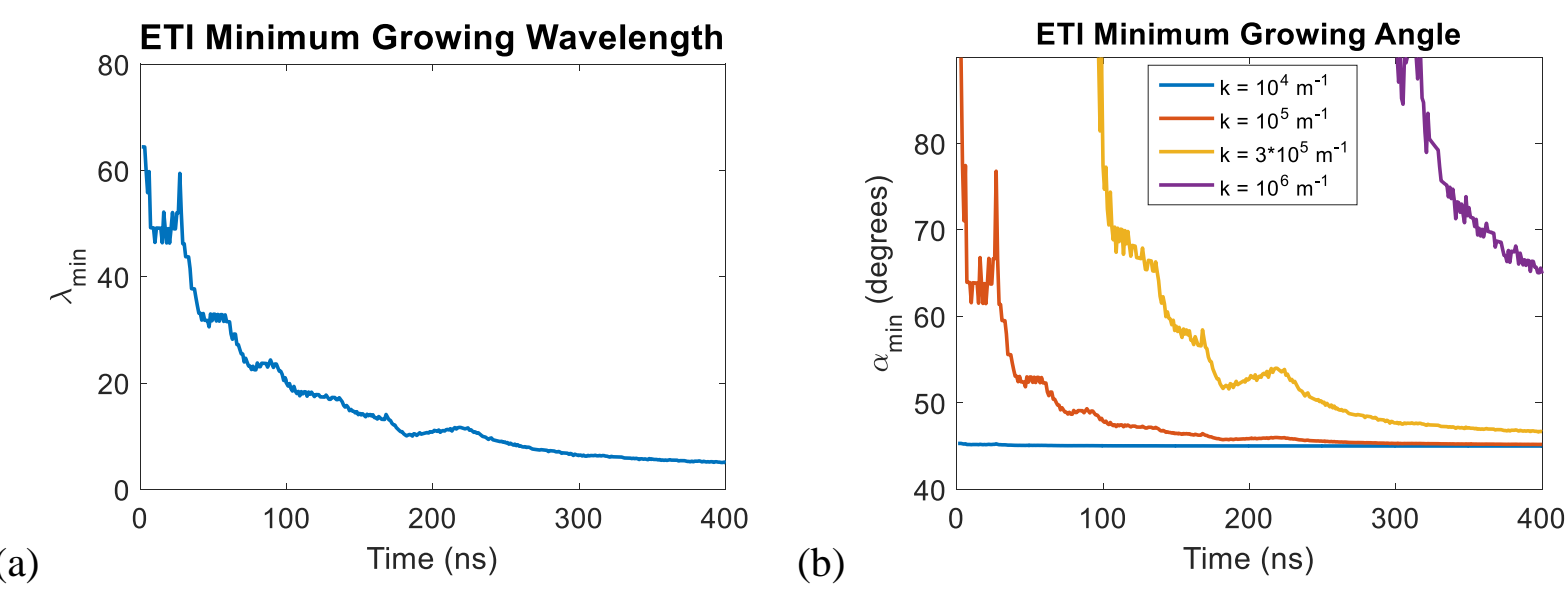

Figure 2.12: Minimum conditions for ETI growth obtained for a $4 \mathrm{kA}, 600 \mathrm{~ns}$ risetime pulse into a $800 \mathrm{~nm} \times 9.0$ $\mathrm{mm}$ x $7.0 \mathrm{~mm}$ aluminum foil. Current density, resistivity, specific heat, density, and thermal conductivity were obtained as in Figure 2.11. These values were input into equations (2.21) and (2.23) to obtain $\lambda_{\min }$ and $\alpha_{\min }$.

\subsection{Effects of Expansion}

While foil expansion can largely be neglected in the solid and liquid phases, significant expansion can occur in the liquid-vapor biphase transition, vapor state, and plasma state. The general case of striation-form ETI was treated by Oreskhin [19], with growth rate

$$
\gamma=\frac{J^{2} \frac{\partial \eta}{\partial T}-k^{2} \kappa+\frac{\rho}{T^{*}}\left(c_{v} \frac{\partial T}{\partial t}-J^{2} \frac{\partial \eta}{\partial \rho}\right)}{\rho c_{v}+\frac{p}{T^{*}}}
$$

Where $T^{*}$ is a modified temperature given by

$$
T^{*}=T\left(\frac{a}{b}+\frac{\gamma^{2}}{k^{2}} \frac{\rho}{b p}\right)
$$

And $a, b$, and $C$ are material-dependent coefficients from the approximate equation of state

$$
p=C \rho^{a} T^{b}
$$

There are two primary effects of the additional terms in (2.40). First, in the biphase liquid-aluminum state, all modes are unstable (there is no maximum growing $k$ ), and lowwavelength modes have large growth rates compared to $\gamma_{m}$ in single-phase liquid. Second, the presence of the $-J^{2} \frac{\partial \eta}{\partial \rho}$ term provides an additional source of growth for the striation mode, since 
resistivity increases with decreasing density in gas and plasma phases. Therefore, the striation mode is able to grow past the point of plasma breakdown where $\frac{\partial \eta}{\partial T}$ becomes negative, and the striation mode only begins to damp when the dependence of resistivity on temperature becomes sufficiently negative to balance the additional growth term. This is an important effect in explaining the ability of ETI to couple with plasma-phase instabilities, such as MRT, helical modes, and sausage modes. 


\section{CHAPTER 3}

\section{Experimental Configuration for Foil and Liner Ablations}

\subsection{Planar Foil Ablations on Atmospheric Pulsed Power Discharges}

\subsubsection{Pulsed power system and diagnostics}

The primary studies of striation ETI performed in this work analyze the growth of temperature perturbations on ablating planar foils. These planar foil ablations were performed using a $20 \mathrm{~J}$ high voltage pulse generator, shown in Figure 3.1. To fire the pulse generator, high voltage is applied to charge a $240 \mathrm{nF}$ capacitor, which is grounded on one terminal and connected to a single-stage spark gap switch on the other. When the voltage across the switch reaches $\sim 13 \mathrm{kV}$, the switch breaks down, delivering a pulse with peak current of $4 \mathrm{kA}$ and $600 \mathrm{~ns}$ risetime. The equivalent series resistance and inductance of the switch/capacitor configuration are $0.28 \Omega$ and $80 \mathrm{nH}$, respectively. Two $\mathrm{HV}$-insulated wires are used to connect the pulse generator output to the load; these wires have total inductances of $401 \mathrm{nH}$ and $463 \mathrm{nH}$. An additional array of resistors with equivalent resistance $0.83 \Omega$ is located between the output of the switch and the load to raise the impedance of the driver, providing a consistent current pulse that is relatively independent of load parameters. A schematic of this circuit is shown in Figure 3.2 . 


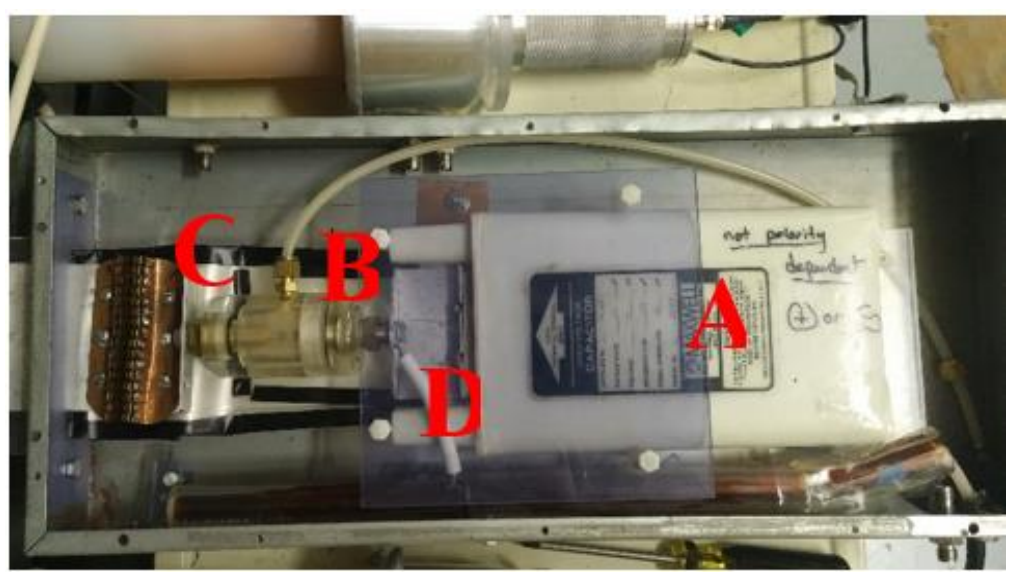

Figure 3.1: Pulse power generator. (A) $240 \mathrm{nF}$ capacitor (B) Atmospheric pressure air spark gap switch (C) $0.83 \Omega$ resistor array (D) High voltage input from power supply. The applied high voltage is slowly increased to $13 \mathrm{kV}$, at which point the spark gap switch spontaneously breaks down and conducts current.

Time-dependent load voltage is measured using two North Star PVM-5 high voltage probes (80 MHz maximum frequency) connected to either side of the load. A Pearson Electronics model 110-A (20 ns minimum usable risetime) wideband current monitor is positioned around the ground-side HV-insulated wire to measure current through the load. One of the PVM-5 probes was factory calibrated immediately prior to the experimental campaign to output $1 \mathrm{~V} / 2 \mathrm{kV}$ through a 50-foot, $50 \Omega$ coaxial cable; the other PVM-5 probe was calibrated directly against this probe and was found to output $1 \mathrm{~V} / 1914 \mathrm{~V}$ through a 50 -foot, $50 \Omega$ cable. The Pearson coil output was connected to a nominal $20 \mathrm{~dB}$ attenuator and $50 \Omega$ terminator; this current-measurement system was calibrated in situ by firing the pulse generator into a short circuit, measuring the charge voltage on the capacitor using the factory-calibrated North Star high voltage probe, and requiring

$$
\frac{1}{2} C V_{0}^{2}=\int_{0}^{t_{\infty}} I(t)^{2} R d t
$$


Where $V_{0}$ is the voltage on the capacitor at switch breakdown, $C$ is the capacitance of the capacitor, $R$ is $1.11 \Omega$ (the total series resistance of the circuit), and $I(t)$ is the current. The calibration factor for the current measurement was found to be $159 \mathrm{~A} / \mathrm{V}$.

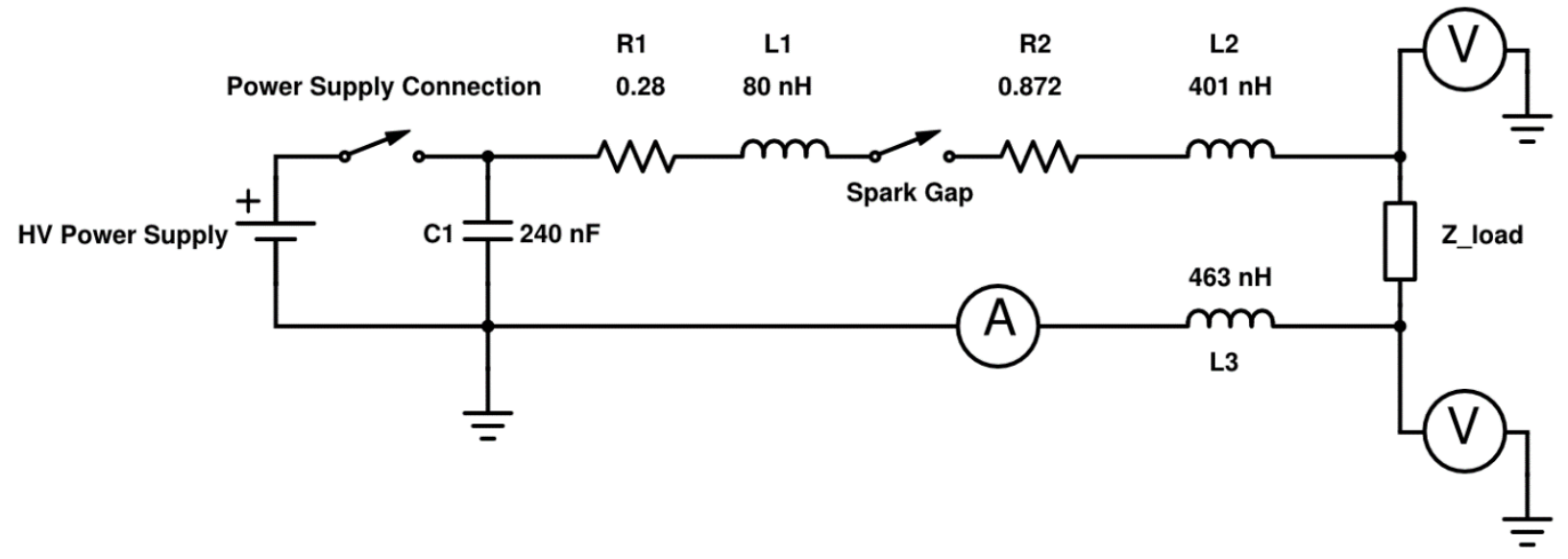

Figure 3.2: Schematic of the pulsed power circuit. Voltage across the load is monitored with the two North Star high voltage probes indicated on the diagram. Current is measured using a Pearson coil placed around the output wire. The load inductance is negligible compared to the high inductances of the circuit; load resistance is measured assuming zero load inductance from the voltage and current measurements.

The load hardware, consisting of two L-shaped aluminum plates mounted on a plastic support structure, is fielded in normal laboratory air under standard atmospheric pressure at room temperature. The plates are separated by a 9-mm gap; foil loads are cut into strips approximately $15 \mathrm{~mm}$ long and placed on the plastic support to span this gap. The aluminum plates each have two slots to accommodate \#8-32 plastic screws, which are used to compress the plates onto the foil to achieve good electrical contact. Each L-bracket is connected to one of the outputs of the pulse generator and to one of the North Star high voltage probes. The assembled load hardware is affixed to optical stands and placed on an array of translation stages to allow controlled movement in the $x^{-}, y^{-}$, and z-directions. Figure 3.3 shows the load hardware with a foil in place. 

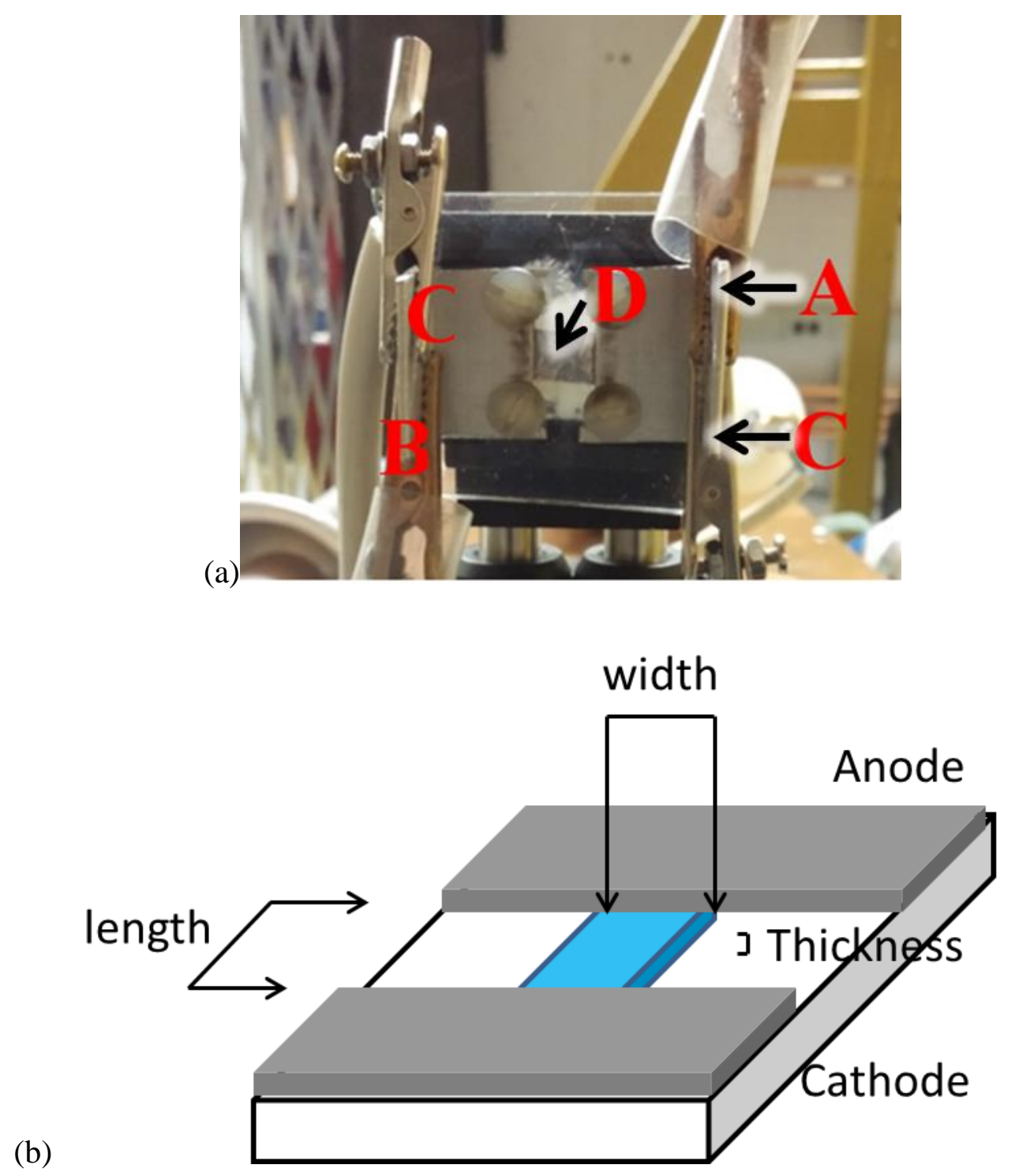

Figure 3.3: (a) Load hardware for atmospheric foil ablations. (A) Connection to pulser output (B) connection to pulser ground side (C) Connection to high voltage probe (D) Foil load. Conventional current flows from left to right.(b) Schematic view of foil with dimensions indicated as referred to in the text.

\subsubsection{Load Properties}

Because the pulse generator outputs nearly constant current pulses, load current density was varied by changing the dimensions or material of the foil loads. The vast majority of the shots utilized ultrathin aluminum foils purchased from Goodfellow. Three thicknesses were used in these experiments: $400 \mathrm{~nm}, 800 \mathrm{~nm}$, and $2.0 \mu \mathrm{m}$. Foil purity is $99.1 \%$, with primary 
impurities consisting of iron $(0.47 \%)$, silicon $(0.16 \%)$, titanium $(0.07 \%)$, and gallium $(0.05 \%)$.

A handful of shots utilized $500 \mathrm{~nm}$ titanium foils, also purchased from Goodfellow. These foils are $99.9 \%$ pure, with $<0.05 \%$ niobium and $<0.01 \%$ other trace elements. Nominal thickness uncertainty for all foils is $25 \%$. Width of foil loads was varied from $2.5 \mathrm{~mm}$ to $10 \mathrm{~mm}$. At a peak current of $4 \mathrm{kA}$, this allowed the investigation of a peak current density parameter space of $2 \times 10^{7} \mathrm{~A} / \mathrm{cm}^{2}$ to $4 \times 10^{8} \mathrm{~A} / \mathrm{cm}^{2}$.

The skin depth for these foils can be calculated from (2.4). For $600 \mathrm{~ns}$, this skin depth is on order 100's of $\mu \mathrm{m}$. Therefore, the foils are electrically thin in thickness, but electrically thick in width. The inductive current distribution can be calculated using a 1-D implementation of the method of moments [32]; the results of this calculation for a sample foil geometry of $800 \mathrm{~nm}$ thickness by $7 \mathrm{~mm}$ width are shown in Figure 3.4 .

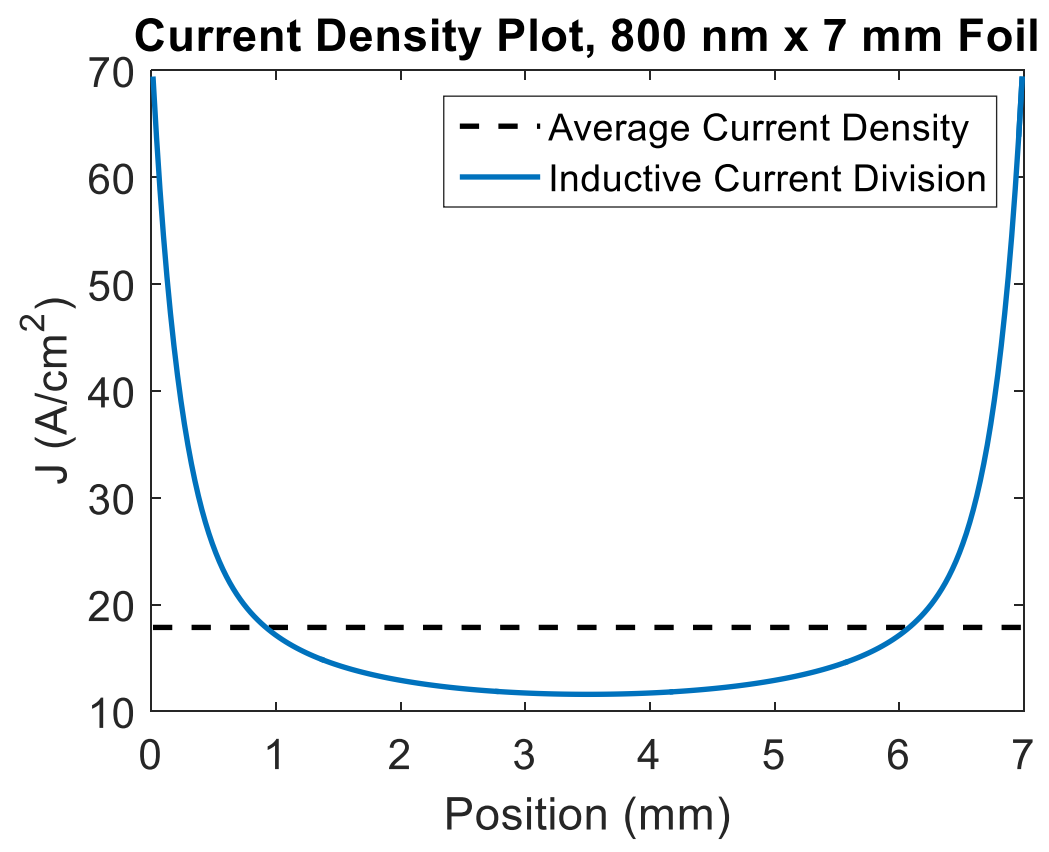

Figure 3.4: Current density distribution calculated from the 1-D method of moments for a thin conducting foil. The calculation was performed for a $600 \mathrm{~ns}$ risetime pulse into an $800 \mathrm{~nm}$ x $7 \mathrm{~mm}$ room temperature aluminum foil with resistivity $2.7 \times 10^{8} \Omega-\mathrm{m}$. For comparison, a uniform current density into the same cross section of aluminum is also plotted. 
From Figure 3.4, it is apparent that the current density in the center of the foil is around $35 \%$ less than the assumed uniform current density, and the current density at the edges is more than a factor of 3 greater. However, this current calculation assumes uniform temperature, and electrothermal effects act in this case to flatten the temperature profile. As the edges of the foil heat up, they increase in resistivity, which is a perturbation in the parallel-to-current direction.

Since $\frac{\partial \eta}{\partial T}>0$ for metals, parallel perturbations are damped. The electrothermal effect appears to dominate the effect of inductive current distribution; temperature measurements described in Section 3.6 do not show significant temperature variation along the width of the foil. Therefore, calculations in this work assume current density to be uniform at the average value given by

$$
J=\frac{I}{w h}
$$

where $w$ is the foil width and $h$ is the thickness.

\subsubsection{Experiments Utilizing Gas Flows or Vacuum}

While most of the planar foil ablation shots were performed in air, several shots were taken at approximate atmospheric pressure in other gas environments. This was achieved by placing a plastic bag over the load, connecting the leads to the pulse generator and voltage probes through holes in the bag, and delivering gas from a gas bottle through a standard $1 / 4$-inch $(6.35 \mathrm{~mm})$ nylon gas line (where the outlet is simply leaks in the plastic bag). Two gases were chosen: argon, for its non-reactivity, and $\mathrm{SF}_{6}$, for its high dielectric strength; a gas fill experiment utilized one of these two gases.

A few planar foil shots were performed in vacuum using the vacuum chamber shown in Figure 3.x. In this configuration, the load hardware is placed against a 4-inch $(10.16 \mathrm{~cm})$ vacuum window to allow access to optical diagnostics. The output of the pulse generator is connected to high-voltage feedthrough ports, which are electrically connected to cables inside 
the vacuum chamber that attach to the load hardware. This changes the electrical properties of the circuit; the new values for L2 and L3 in Figure 3.2 are $651 \mathrm{nH}$ and $715 \mathrm{nH}$, respectively.

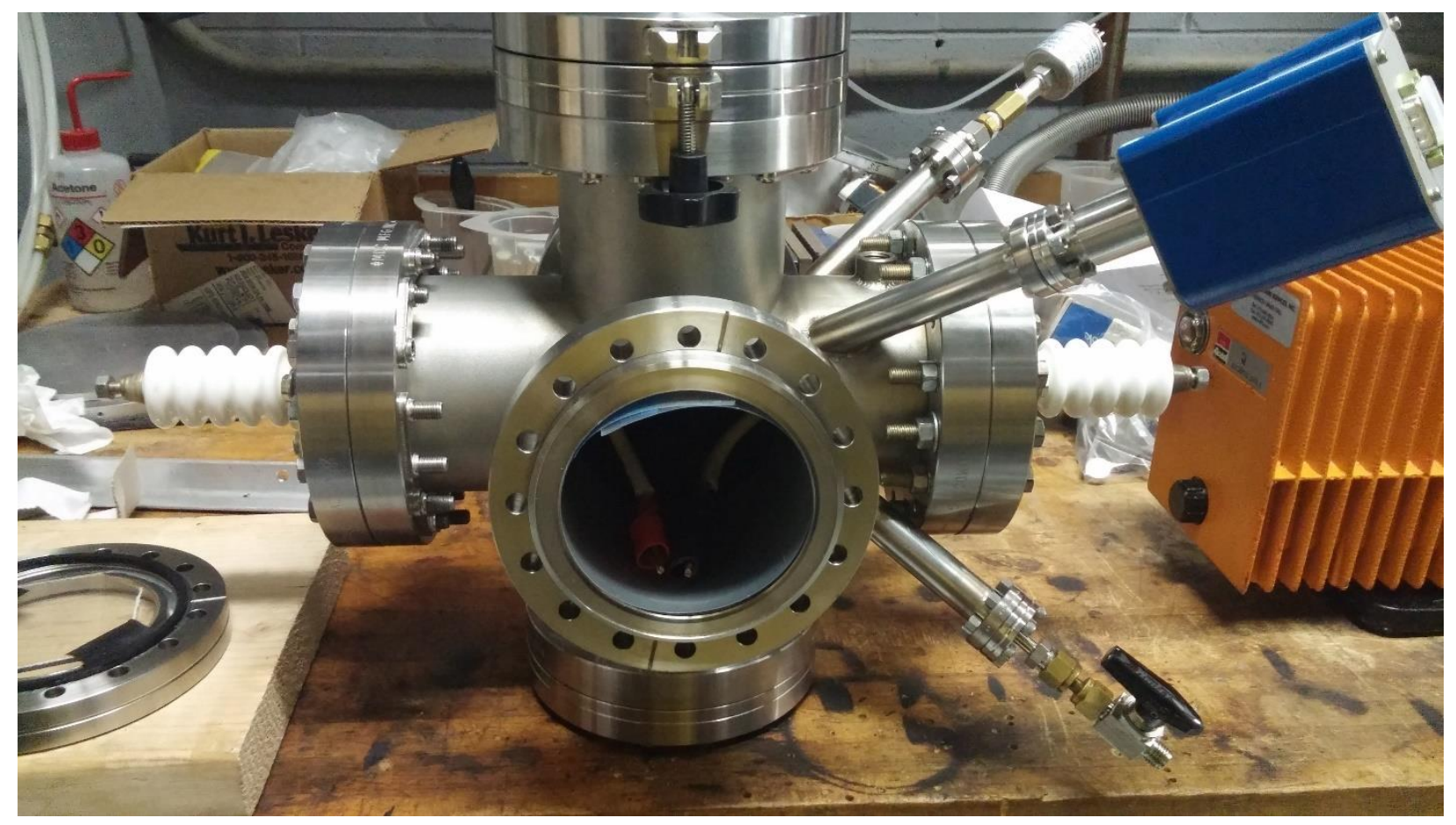

Figure 3.5: Vacuum chamber used for vacuum planar foil shots.

\subsection{Visible Spectrum Diagnostics}

\subsubsection{Ultrafast Framing Camera Imaging System}

The primary optical diagnostic fielded in these experiments is an Invisible Vision $\bigodot$ Ultra UHSi 12-frame intensified charge coupled device camera (ICCD). This camera is capable of capturing 12 images per shot with a frame rate of up to $2 \times 10^{8} \mathrm{~s}^{-1}$ and minimum exposure time of 5 ns. An Edmund Optics VZM-1000 Zoom Imaging Lens is mounted on the camera to provide optical magnification ranging from $2.5 \mathrm{x}$ to $10 \mathrm{x}$. Spatial resolution of the imaging system was measured by imaging a backlit 1951 USAF resolution test target. The minimum resolvable wavelength at the in-focus imaging distance for the maximum 10x magnification was found to be 
$2.5 \mu \mathrm{m}\left(4 \times 10^{5}\right.$ line pairs per meter). Figure 3.6 shows the camera with the zoom imaging lens positioned to image a foil target.

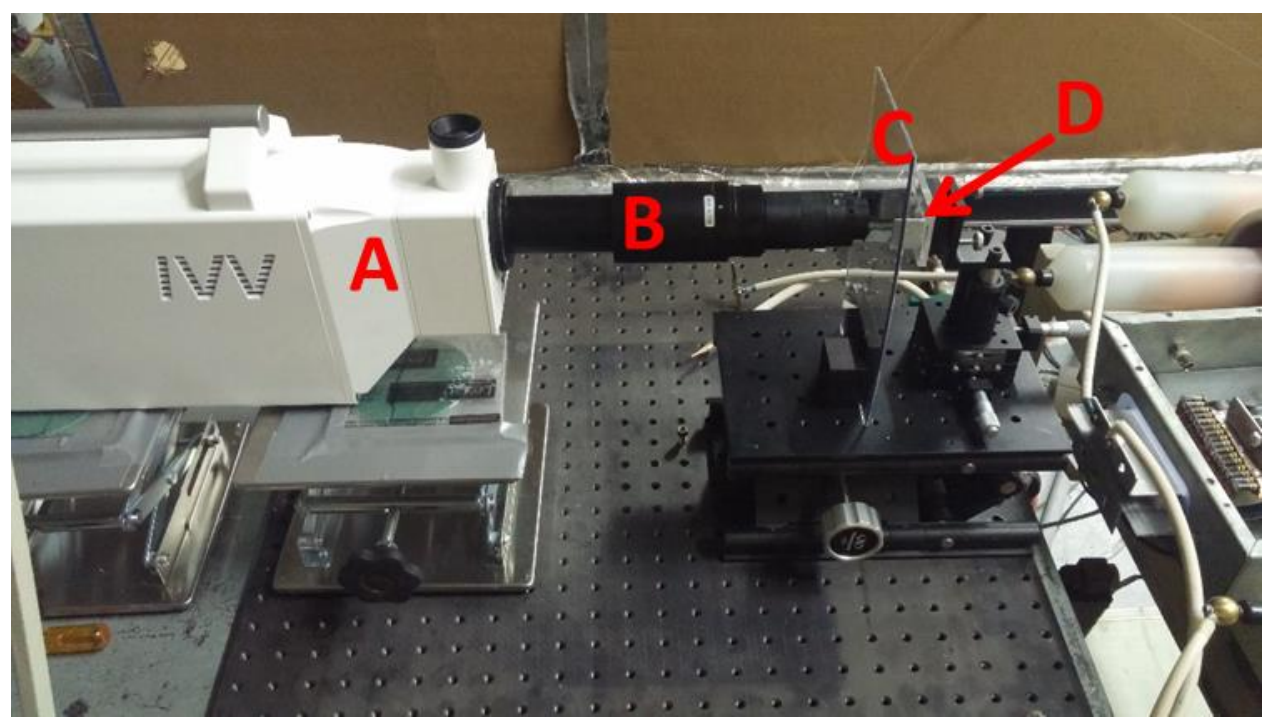

Figure 3.6: Ultrafast framing camera (A) with zoom imaging lens (B) positioned to image a foil load (D). A sheet of Plexiglas $(\mathrm{C})$ is positioned between the load and the imaging lens to act as a sacrificial window, preventing deposition on the lens.

Self-emission from the ablating foils was imaged as a function of time using the framing camera for each shot. As the ICCD is designed for use primarily in the visible portion of the spectrum, self-emission in the visible wavelengths dominates the camera response. A response vs. wavelength curve was supplied by the manufacturer [33] and is shown in Figure 3.7. 


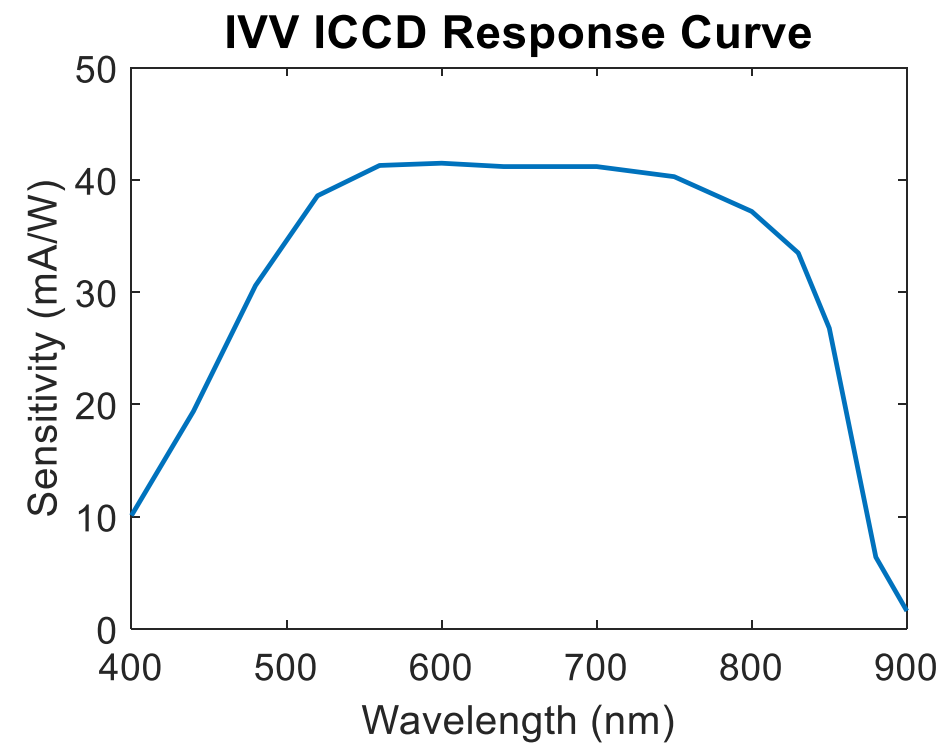

Figure 3.7: ICCD response as a function of wavelength.

For each shot, the load translation stages were used to make fine adjustments to load position relative to the camera to optimize the focus and to fix the camera imaging window on the center of the foil. The center of the foil was maintained as the region of interest to minimize the contribution of edge effects, such as contact resistance with the electrodes or the naturally rough foil boundaries that resulted from cutting the thin, fragile foils. A pre-shot image of each foil was taken by selecting a long ( 10 ms) exposure on the camera and illuminating the surface of the foil with a high intensity LED flashlight. Figure 3.8 shows a sample pre-shot image. Microscopic foil nonuniformities, such as wrinkles and impurity inclusions, are visible on these pre-shot images. 


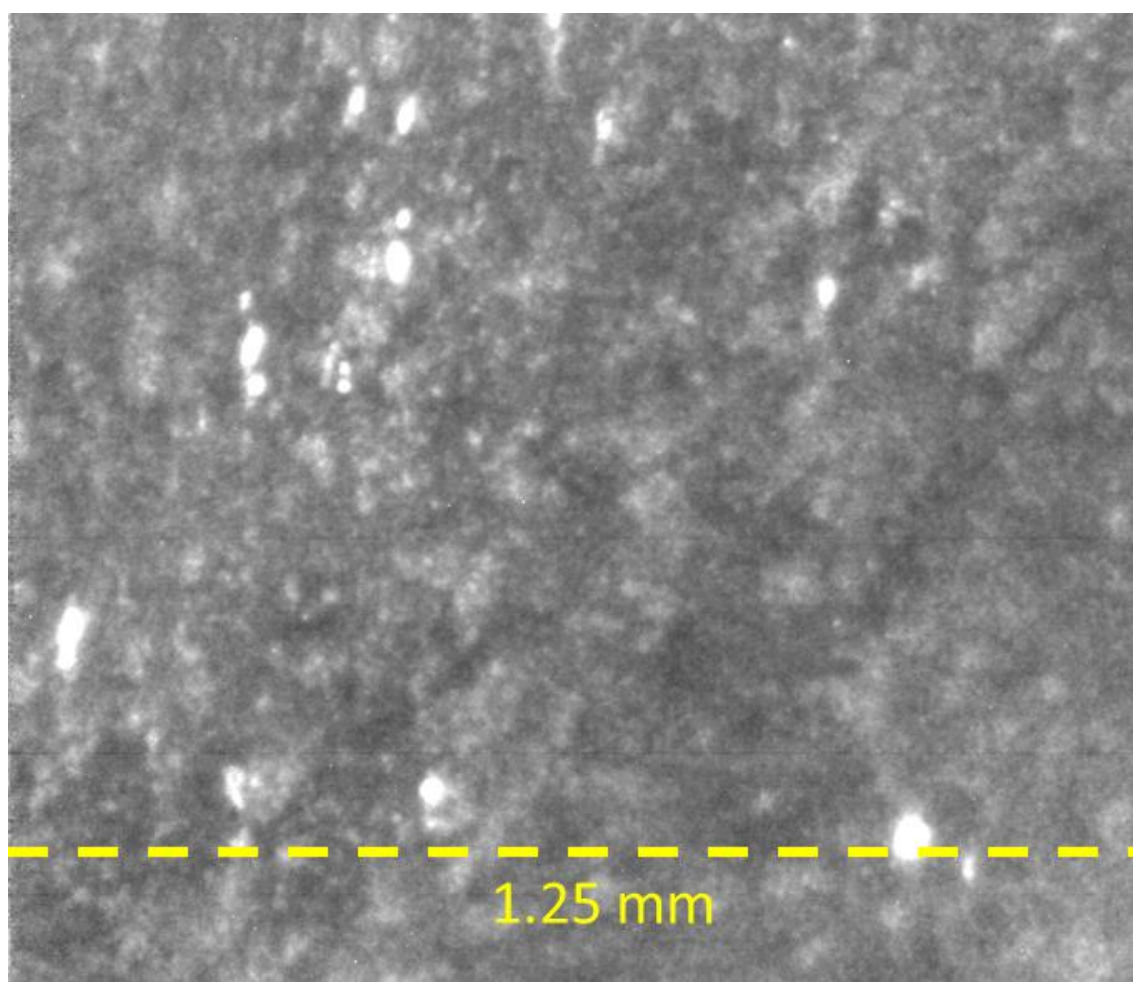

Figure 3.8: Pre-shot image of shot E-016, showing typical foil surface characteristics. Some of the bright spots result from sharp points on the surface of the foil resulting from folds; others result from more highly reflective inclusions.

\subsubsection{Time-Integrated Spectroscopy and Time-Resolved Emission Measurements}

Several shots utilized time-integrated spectroscopy to obtain information about the composition of plasma formed during foil ablation. On these shots, the framing camera was replaced with an optical system shown in Figure 3.9. A convex lens with $10 \mathrm{~cm}$ focal length was placed $50 \mathrm{~cm}$ from the foil, and a bare Poly(methyl methacrylate) (PMMA) fiber optic cable was positioned at the image distance of $12.5 \mathrm{~cm}$ from the lens, resulting in a magnification of $1 / 4$. The other end of the fiber is coupled through a 1:1.7 achromat lens into a Princeton Instruments Acton SP-750i spectrometer, which is digitally imaged using a Princeton Instruments gated ICCD. Proportional response as a function of wavelength was calibrated using a $40 \mathrm{~W}$, OL-245 
standard of spectral irradiance, and wavelength calibration was performed using neon, argon, and mercury lamps for each grating.

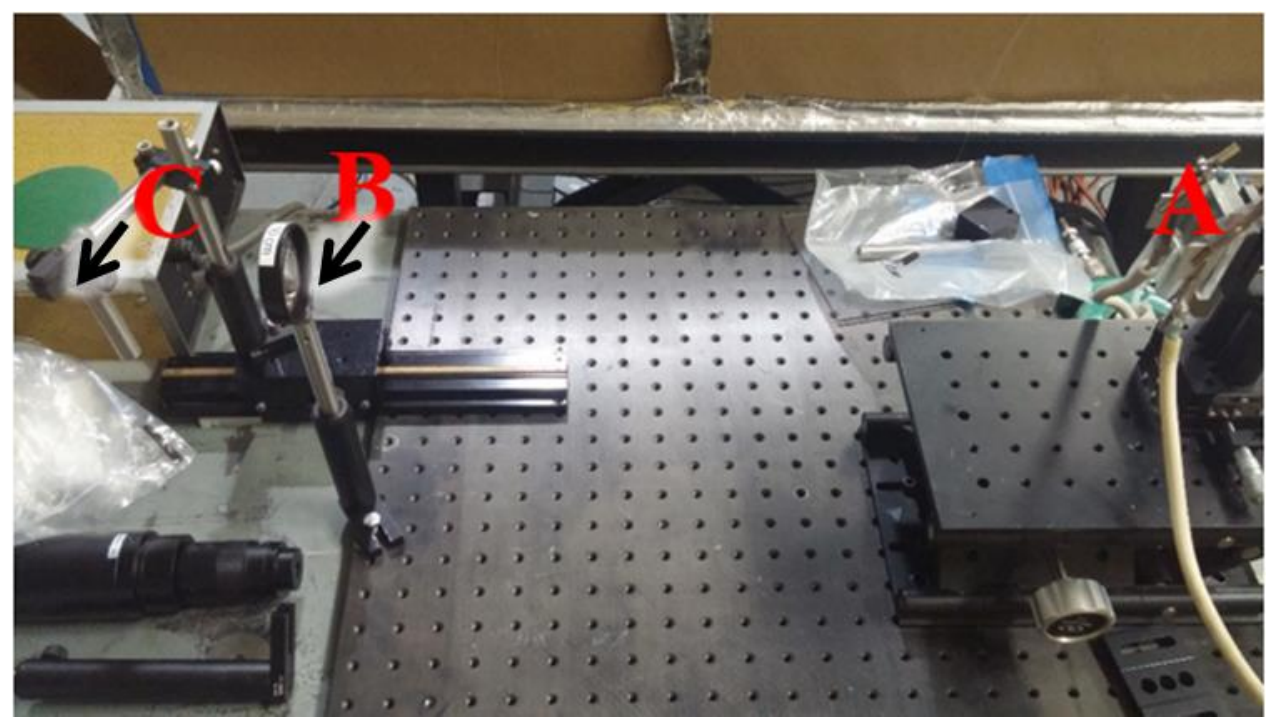

Figure 3.9: Fiber imaging setup for optical emission spectroscopy and time-resolved PMT measurements. (A) Foil load hardware (B) $10 \mathrm{~cm}$ focal length lens (C) PMMA fiber optic.

The same optical system was also used to field time-resolved light emission measurements by connecting the output of the fiber optic to a photomultiplier tube (PMT). These measurements were used to determine the time at which sections of the foil reached the point of breakdown with higher temporal accuracy than the framing camera provided. Breakdown times were compared to available voltage and current data to identify electrical features corresponding to foil explosion. An example voltage-current-PMT signal plot is shown in Figure 3.10. 


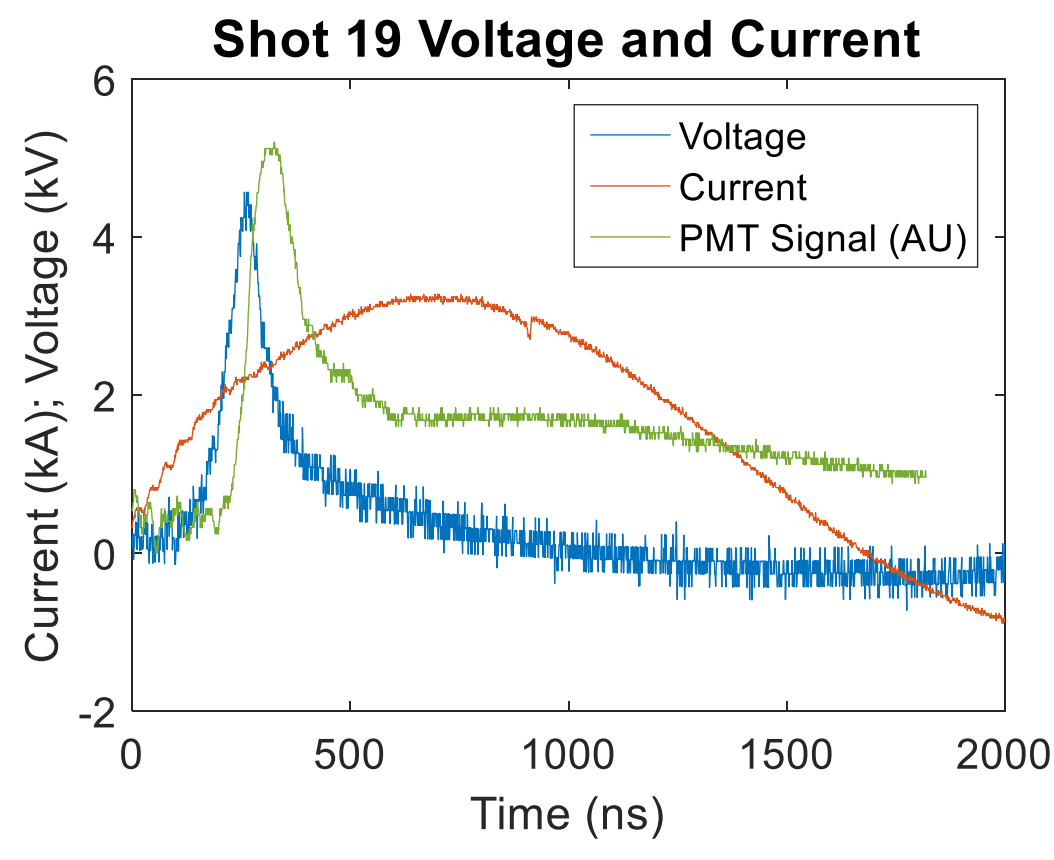

Figure 3.10: PMT signal plotted alongside measured load voltage and current. The peak of the voltage spike lines up with the start of light emission; this is a consistent feature on shots fielding the PMT.

\subsection{Time-Resolved Temperature Measurements}

As the primary purpose of the planar foil ablations was to observe the electrothermal instability that grows in the solid and liquid phases, it was necessary to develop a method to correlate the time-resolved light emission measurements on the framing camera with surface temperature. Blackbody-like thermal emission was assumed to be the dominant mechanism responsible for the self-emission detected by the camera. This assumption is addressed in Chapter 5, where it will be shown that the effects of competing light production mechanisms such as line emission, continuum emission from Bremsstrahlung radiation, and continuum emission from recombination are small. In this limit, temperature can be computed from camera response (which is linear with incident irradiance at values below saturation [33]) by determining the integral blackbody emission for a given temperature, adjusting for the wavelength-dependent response of the camera, and calibrating against a blackbody source of known temperature. This 
process provides 12 -frame temperature imaging with the same special and temporal resolution as the framing camera system. Figure 3.11 shows sample blackbody spectra (in proportional arbitrary units) for several temperatures in the temperature regime of ablating foils.

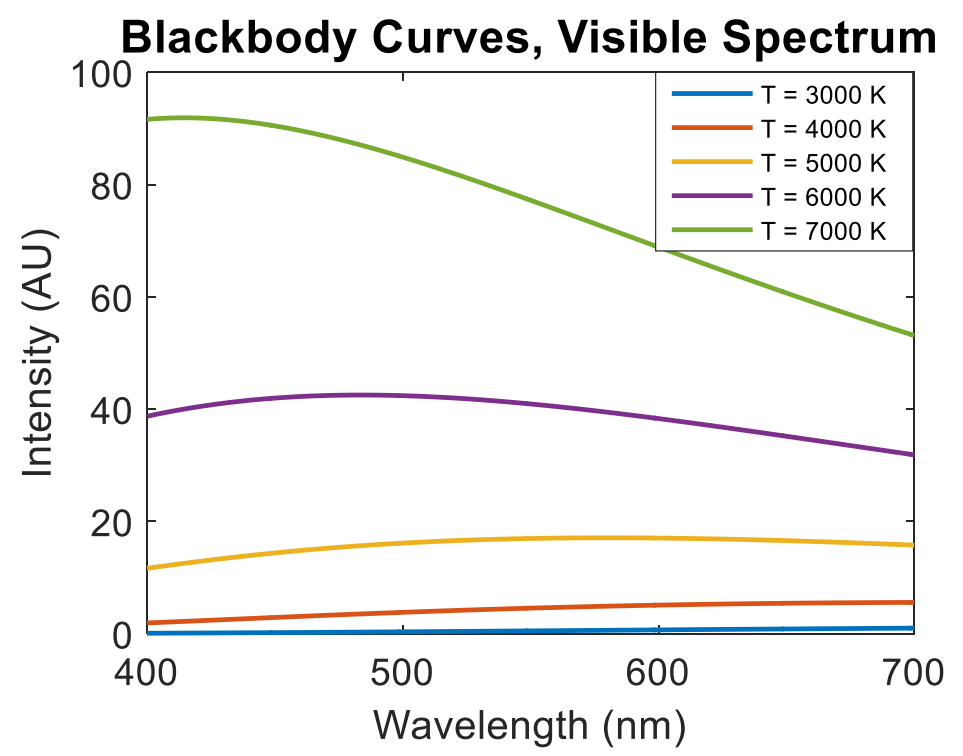

Figure 3.11: Blackbody emission in the visible spectrum for various temperatures, scaled in proportional arbitrary units.

To calibrate camera response, a 2977 K OL-245 tungsten standard of spectral irradiance was placed at the in-focus distance from the lens on 10x magnification and imaged using the framing camera. Tungsten at the temperature of the calibration source radiates to good approximation as a graybody with emissivity 0.43 [34]. An image of the calibration source is shown in Figure 3.12. Figure 3.12 shows the coiled tungsten filament; the face-on sections of the filament were assumed to be at $2977 \mathrm{~K}$. The edges of the filament are brighter than the faceon surfaces; this is because they are both radiating with emissivity 0.43 and reflecting emission from other surfaces (without line-of-sight to the camera) at reflectivity 0.57 . 


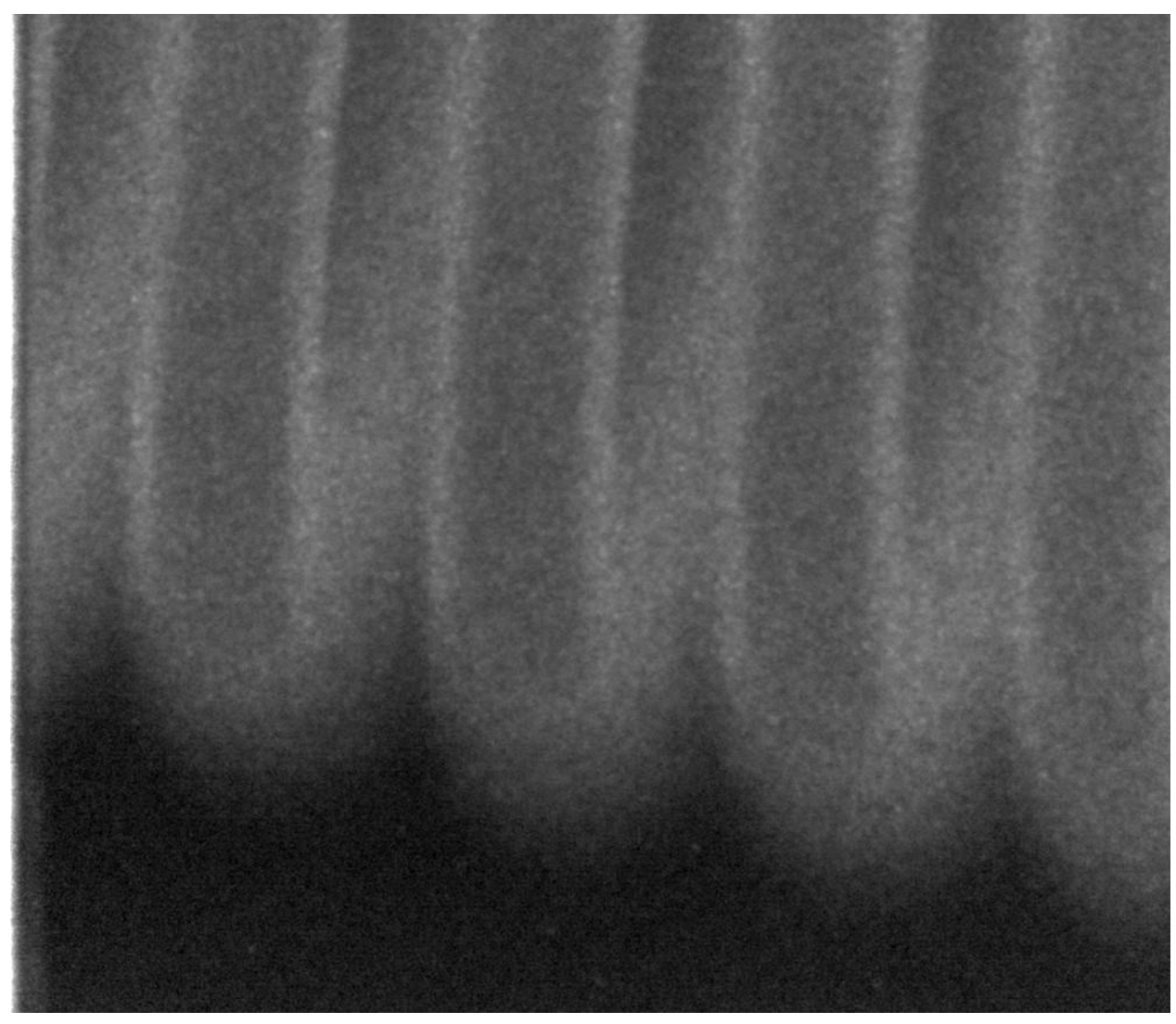

Figure 3.12: Tungsten calibration source at $2977 \mathrm{~K}$, imaged with $600 \mathrm{~ns}$ exposure at 10x magnification on the framing camera.

The image shown in Figure 3.12 was used to determine the 0-255 grayscale camera response (per nanosecond) associated with a blackbody radiating at $2977 \mathrm{~K}$. This value was found to be $0.33 \mathrm{~ns}^{-1}$. Blackbody spectra at temperatures from $2000 \mathrm{~K}$ to $10000 \mathrm{~K}$ were multiplied by the camera response given in Figure 3.7, expressed as a percentage of peak response, and integrated over wavelength to give proportional values of camera response as a function of temperature. Proportional camera response was then normalized to $0.33 \mathrm{~ns}^{-1}$ at 2977 $\mathrm{K}$ to give an absolute calibration of camera response as a function of blackbody emitter temperature at 10x magnification. Figure 3.13 shows the results of this calibration for various integration times. To extend this calibration to other magnification values, the in-focus distance 
was measured at magnifications of 10x, 5x, and 2.5x, and was found to be $35 \mathrm{~mm}, 34 \mathrm{~mm}$, and $32 \mathrm{~mm}$, respectively. The response to a given temperature at magnifications other than $10 \mathrm{x}$ is then given by

$$
\frac{R}{R_{10}}=\left(\frac{10}{m}\right)^{2}\left(\frac{d}{d_{10}}\right)^{2}
$$

where $R$ is the camera response on the $0-255$ scale, $R_{10}$ is the camera response for $10 \mathrm{x}$ magnification, $m$ is the magnification, $d$ is the in-focus distance, and $d_{10}$ is the $35 \mathrm{~mm}$ in-focus distance for 10x magnification. In (3.3), the $\left(\frac{10}{m}\right)^{2}$ factor is due to the increased collection area per pixel, and the $\left(\frac{d}{d_{10}}\right)^{2}$ factor is due to the $\frac{1}{r^{2}}$ dependence of intensity on distance from the source. The noise floor of the camera corresponds to a response of approximately 10, and above a response of 245 the linearity of the camera response becomes unreliable, so the temperature range that can be reliably measured by the camera is a function of both exposure time and magnification. Additionally, the camera response was found to vary on the order of 5-10\% from image to image on the constant temperature calibration source, placing an approximated $10 \%$ uncertainty on temperature measurements obtained from camera response. 


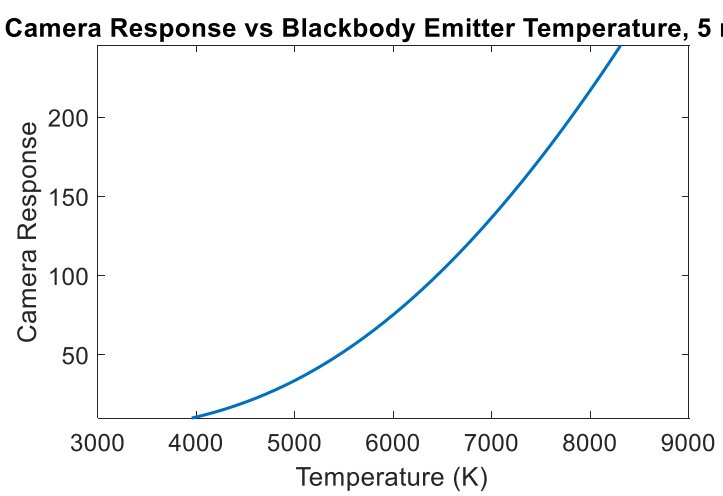

(a)

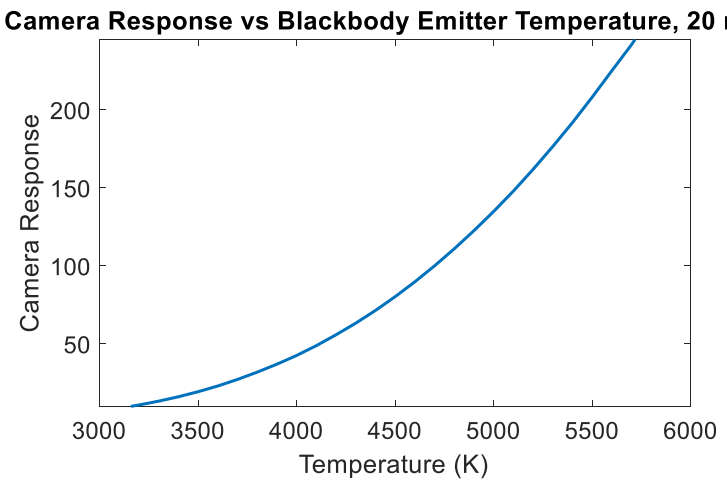

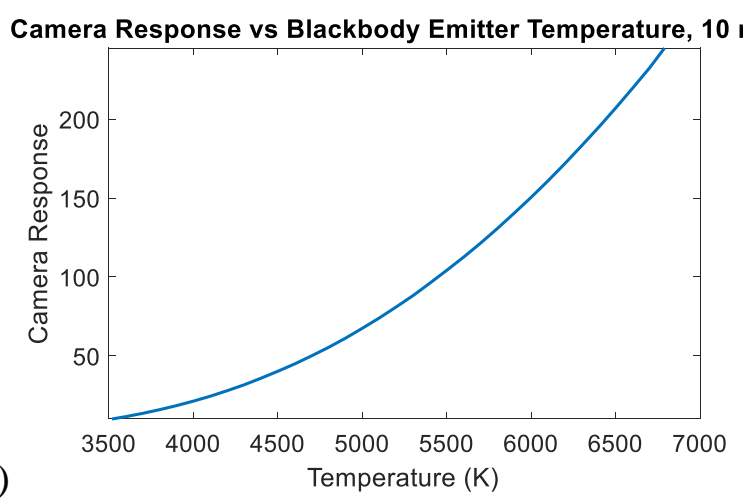

(b)

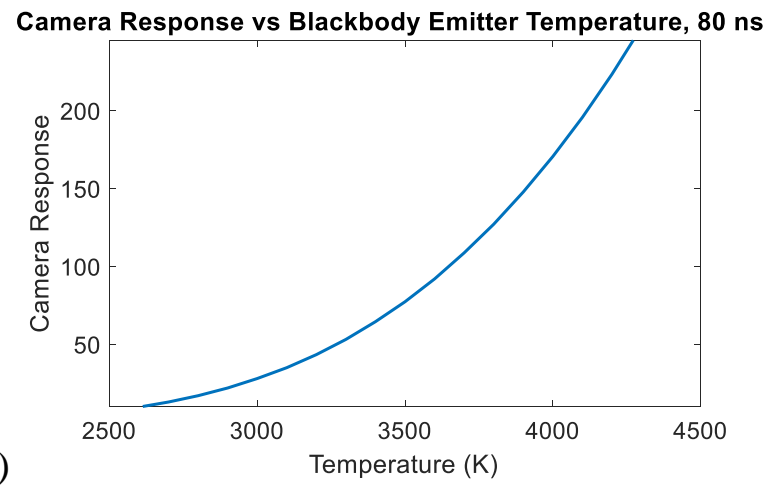

Figure 3.13: Camera response as a function of blackbody emitter temperature for exposure times of (a) $5 \mathrm{~ns}$ (b) 10 ns (c) $20 \mathrm{~ns}$ and (d) $80 \mathrm{~ns}$. Camera response values below 10 are considered below the noise floor, and values above 245 are outside the linear regime, limiting the effective temperature range that can be measured for a given exposure time.

The final factor required to correlate camera response with emitting surface temperature is the emissivity of aluminum in the temperature range of interest. While aluminum emissivity has been experimentally measured at temperatures below $1500 \mathrm{~K}$ in the liquid phase [35] little information exists on aluminum at temperatures in the $2000 \mathrm{~K}$ to $10,000 \mathrm{~K}$ range. Attempts to measure emissivity of aluminum vapor in this temperature range at Sandia National Labs [36] were inconclusive, estimating emissivity of aluminum vapor in this temperature range to be nearly 1 . For this work, emissivity of aluminum vapor was assumed to be 1 ; this assumption will be revisited in greater detail in Chapter 5. Assuming an emissivity of 1 places a lower bound on the temperature of the emitter; varying emissivity to values as low as 0.4 (a reasonable estimate 
for various other metals with higher melting points around $3000 \mathrm{~K}$ [34]) only changed measured temperature by a few hundred Kelvin, which is on the order of (and usually smaller than) the intrinsic $10 \%$ error of the temperature measurements.

\subsection{Cylindrical Liner Implosions and Explosions}

While the primary purpose of the planar foil ablation experiments was to investigate the growth of ETI as a temperature perturbation, ablations of cylindrical liners were also performed to obtain information on the coupling of ETI to plasma instabilities. In order to couple sufficient energy into the load to drive physics well into the plasma phase, these experiments were performed on the Michigan Accelerator for Inductive Z-Pinch Experiments, a 1 MA linear transformer driver that is described in detail in Chapter 4.

The liner fabrication process is described in Reference [37]. Foils of varied materials were cut into $2.1 \mathrm{~cm}$ by $1.5 \mathrm{~cm}$ strips and wrapped into a liner geometry around an insulating support structure of total length $3.4 \mathrm{~cm}$ and diameter $6.3 \mathrm{~mm}$. A single layer of aluminum tape was wrapped around the top $1 \mathrm{~cm}$ and bottom $1.4 \mathrm{~cm}$ of the support, providing a conducting path from the foil to the anode and cathode of the load hardware. Two types of support structures were fielded, shown in Figure 3.14: solid support structures and dumbbell-shaped support structures. For sufficiently thin liners (linear mass less than $\sim 0.50 \mathrm{mg} / \mathrm{cm}$ ), the dumbbell supports allow a section of the liner to implode, destabilizing the liner to the magneto RayleighTaylor (MRT), kink, and sausage modes [38, 39]. The solid plastic supports prevent implosion and allow only outward acceleration, causing the liner to become stable to MRT while remaining unstable to kink and sausage modes. 
(a)

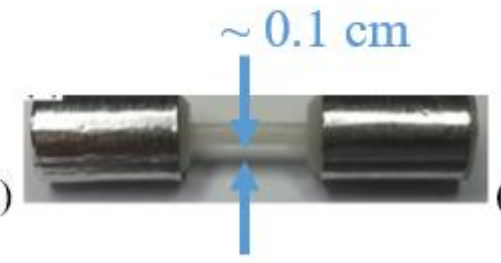

(b)

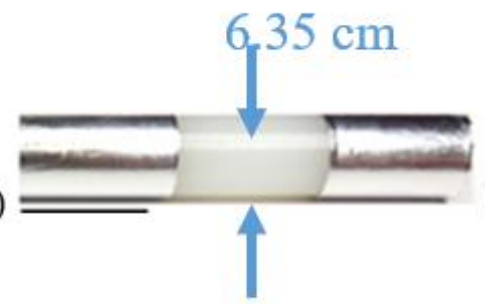

(c)

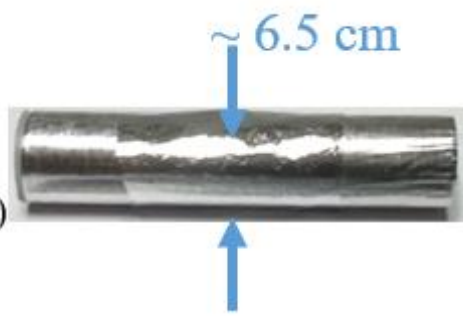

Figure 3.14: Support structures used for liner ablation experiments. (a) Dumbbell support allowing a $1 \mathrm{~cm}$ section of the liner to implode due to magnetic pressure (b) Solid support preventing implosion (c) Assembled liner with aluminum foil in place. The ends of the support structures are wrapped with aluminum tape to allow electrical contact to the anode and cathode; the $1.5 \mathrm{~cm}$ section of foil spans the $1 \mathrm{~cm}$ gap between the tape and makes contact on both sides. All liners are $3.4 \mathrm{~cm}$ in total length.

Foils used on the liner experiments included the $400 \mathrm{~nm}$ aluminum $(0.23 \mathrm{mg} / \mathrm{cm}$ in the liner geometry) and $500 \mathrm{~nm}$ titanium $(1.72 \mathrm{mg} / \mathrm{cm})$ described in Section 3.2. Additionally, a process was developed at the University of Michigan Lurie Nanofabrication Facility (LNF) to fabricate dielectric-coated foils. In this process, the EnerJet electron beam evaporator is used to drive physical vapor deposition of thin (100 nm to $400 \mathrm{~nm}$ ) layers of metals on Chemplex SpectroMembrane Ultra-Polyester films $\left(1.5 \mu \mathrm{m}\right.$ thickness, $0.14 \mathrm{mg} / \mathrm{cm}^{2}$ areal mass density, 0.29 $\mathrm{mg} / \mathrm{cm}$ linear mass density in the liner geometry). The materials and thicknesses chosen for deposition were aluminum at $200 \mathrm{~nm}$ and $400 \mathrm{~nm}(0.41$ and $0.52 \mathrm{mg} / \mathrm{cm}$ total linear mass density, respectively) and titanium at $125 \mathrm{~nm}$ and $250 \mathrm{~mm}$ (also 0.41 and $0.52 \mathrm{mg} / \mathrm{cm}$, respectively).

Diagnostics fielded on the liner implosion experiments included differential output B-dot current monitors [40, 41] and 12-frame gated load imaging using the framing camera described in section 3.1. The optical system for the framing camera on the LTD consisted of a switchyard of lenses and mirrors [42] designed to image the load at approximately 1x magnification through a $1 \mathrm{~nm}$ FWHM $532 \mathrm{~nm}$ line filter. The resolution of this optical system was limited to approximately $75 \mu \mathrm{m}$ but allowed for a viewing window of greater than $1 \mathrm{~cm} \times 1 \mathrm{~cm}$. On some 
shots, a $2 \mathrm{~ns}$ pulse duration, frequency-doubled, $532 \mathrm{~nm} \mathrm{Nd:Yag} \mathrm{laser} \mathrm{was} \mathrm{used} \mathrm{to} \mathrm{backlight} \mathrm{the}$ load. The laser intensity was of the order of self-emission from the ablating liner plasma at 532 $\mathrm{nm}$, generating images with simultaneous contribution from self-emission and visible shadowgraphy. To synchronize the laser with the 12 camera frames, a single $2 \mathrm{~ns}$ pulse is split into many collinear beams using a $3.05 \mathrm{~m}$ resonating cavity with two $95 / 5 \%$ beam splitters, shown in Figure 3.15 [43]. The total length of the cavity is $3.05 \mathrm{~m}$, corresponding to a $10 \mathrm{~ns}$ delay between pulses. The first beam splitter allows the laser pulse to enter the cavity, and the second beam splitter directs the train of pulses through the target chamber and to the imaging system. Due to the two beam splitters, the intensity of each subsequent pulse is reduced by $\sim 10 \%$. This system allows the tracking of individual features from the same angle of incidence in both shadowgraphy and self-emission with 12-frames over a window of $110 \mathrm{~ns}$.

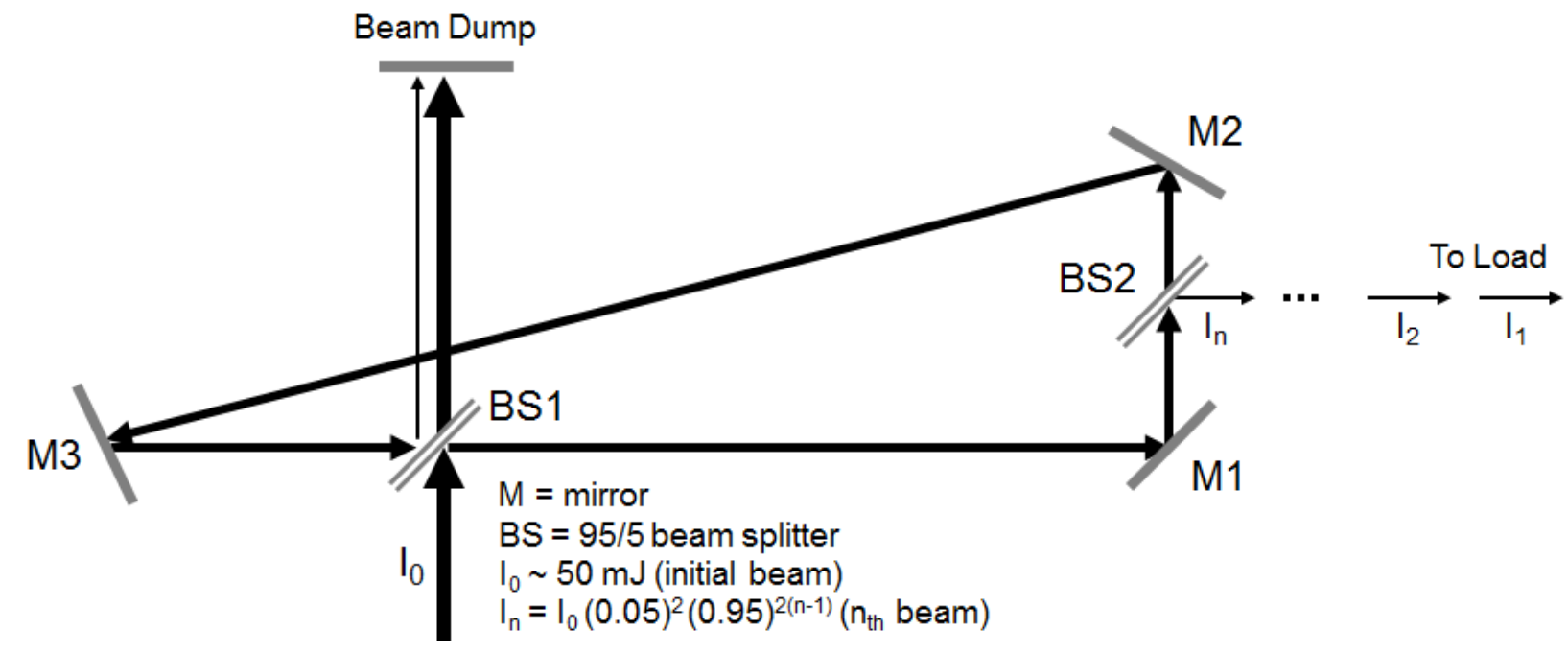

Figure 3.15: Framing camera laser shadowgraphy system. A single 2 ns laser pulse is split into multiple collinear pulses, spaced temporally by $10 \mathrm{~ns}$, using a resonating cavity consisting of mirrors M1, M2, and M3. Beam splitter BS1 inputs 5\% of the initial 2 ns pulse into the cavity and retains $95 \%$ of each subsequent pulse in the cavity, dumping the rest of the laser energy into the beam dump. Beam splitter BS2 picks off 5\% of the beam energy and sends it through the load, providing the backlight for a shadowgraph image. 


\section{CHAPTER 4}

\section{Analysis of the MAIZE Linear Transformer Driver with Dynamic Loads}

The linear transformer driver (LTD) is a compact pulsed power technology that has recently received attention as an alternative to the conventionally used Marx-driven accelerator [44-46]. The low driver-side impedance of LTDs enables fast risetimes without pulse shaping; this property, along with the small footprint and ease of constructing an inductive voltage adding configuration, makes the LTD an attractive technology for constructing next generation pulsed power machines [7, 8], such as the proposed Z-300 and Z-800 facilities at Sandia National Laboratories [9]. However, the low impedance of a single-stage LTD causes the output current and voltage to depend strongly on the properties of the load [47]. This chapter addresses the effects of this load-dependent behavior on the Michigan Accelerator for Inductive Z-Pinch Experiments (MAIZE), a 1-MA LTD at the University of Michigan. An inductance diagnostic based on the response of measured current to load impedance changes is demonstrated for a campaign of planar wire array implosions conducted on MAIZE (described in Section 4.4) and for a series of cylindrical liner implosions (described in Section 4.5).

\subsection{The MAIZE Facility}

The MAIZE facility consists of a single-stage, $0.1 \Omega$ LTD capable of driving 1 MA with 100 ns risetime into a matched, non-inductive load. The generator section consists of 40 bricks, where each brick consists of two capacitors charged to opposite polarities connected in series to 
a six-gap, sequence multi-electrode spark-gap switch. Operating parameters of the six-gap switches used in MAIZE have been analyzed in Reference [48]. A vacuum chamber $1 \mathrm{~m}$ in diameter is positioned inside the generator section to house the load and associated diagnostics [46]. The driver section is directly connected to the load through a coaxial-to-radial magnetically insulated transmission line (MITL). Current measurements are obtained by averaging signals from four B-dot probes positioned in azimuthally symmetric locations in the MITL approximately $0.4 \mathrm{~m}$ radially from the center of the load region; a fifth B-dot probe located at a radial position of approximately $0.35 \mathrm{~m}$ was also fielded for some of the shots. The estimated uncertainty of each B-dot signal obtained from repeated current measurements into a constant resistive load is 5\%, so averaging all four B-dots measures the current with an error of approximately $2.5 \%$. A cutaway schematic view of the fully assembled MAIZE is shown in Figure 4.1. Additional details on single-stage LTD operation and the MAIZE facility are available in other publications [42, 46, 47, 49, 50].

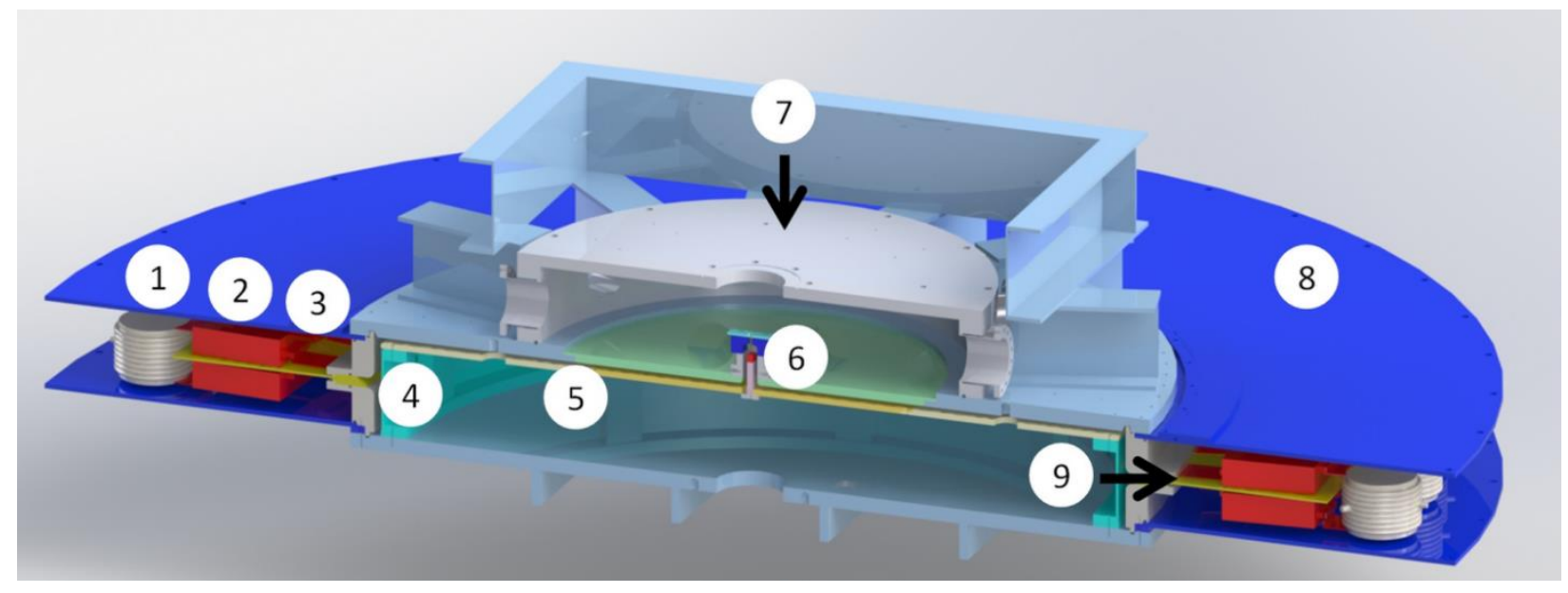

Figure 4.1: Three dimensional model of the assembled MAIZE LTD. (1) Spark gap switch; (2) $40 \mathrm{nF}$ capacitor; (3) Iron core region (core not pictured); (4) coaxial transmission line section; (5) Radial transmission line section; (6) Load region; (7) Vacuum chamber; (8) Oil chamber; (9) High voltage insulator. 


\subsection{Standard Operation MAIZE Circuit Model}

Figure 4.2 shows the standard LTD circuit model used to represent MAIZE. This circuit is similar to the one used to model single-stage LTDs at Sandia National Labs $[44,45,50]$, accounting for the effect of the iron cores as an approximately constant equivalent resistance in parallel with the load [51], and modified to include the spatially-dependent impedance of the transmission line as a series of discrete transmission line elements. The impact of the transmission line usually cannot be neglected because the integral impedance of the transmission line is on the order of the impedance of the driver and can even exceed the impedance of the driver if the load hardware is designed to join with the radial transmission line at a small radius. Taking the assumption that all switches are triggered simultaneously, the capacitors and switches are lumped together into a driver-side equivalent resistance $\left(\mathrm{R}_{\mathrm{gen}}=16.5 \mathrm{~m} \Omega\right)$, inductance $\left(\mathrm{L}_{\mathrm{gen}}=\right.$ $6.0 \mathrm{nH})$, and capacitance $\left(\mathrm{C}_{\mathrm{gen}}=0.800 \mu \mathrm{F}\right)$. The equivalent core resistance has, in general, been observed to change as a function of machine age; at the time of the experiments presented in this work $\mathrm{R}_{\text {core }}=0.9 \Omega$.

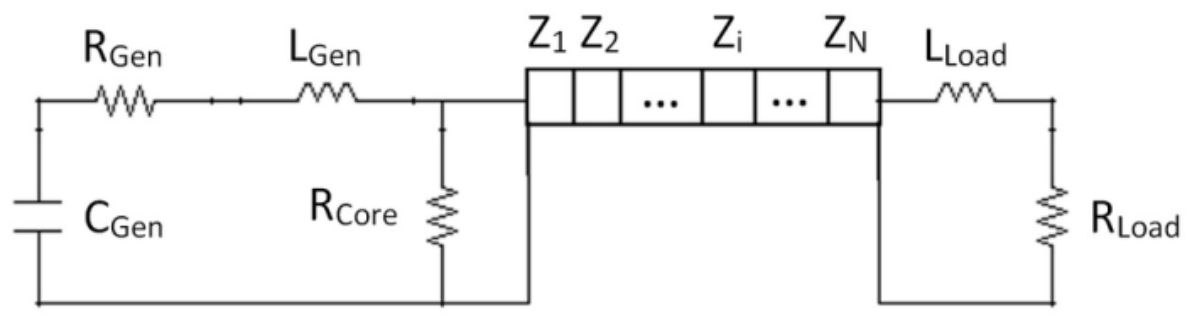

Figure 4.2: Schematic of LTD circuit. $R_{G e n}, L_{G e n}$, and $C_{G e n}$ refer to the lumped resistance, inductance, and capacitance of the 40 parallel bricks. $R_{\text {Core }}$ refers to the parallel resistance of the iron cores due to eddy currents, which dominates core behavior provided magnetic saturation is not reached. $L_{\text {Load }}$ and $R_{\text {Load }}$ are the inductance and resistance of the load. The transmission line is represented as $\mathrm{N}$ discrete elements, each with characteristic impedance $Z_{i}$.

When the charging voltage is specified as an initial condition on the equivalent capacitance of the driver, the system of equations governing voltage and current at each circuit 
element is closed for a given set of load parameters. These equations are Kirchoff's current and voltage laws, the voltage-current relations for capacitive, resistive, and inductive elements, and the telegrapher's equations for a radial transmission line,

$$
\begin{gathered}
\frac{\partial V}{\partial r}=-L^{\prime} \frac{\partial I}{\partial t}=-\frac{\mu_{0} h}{2 \pi r} \frac{\partial I}{\partial t} \\
\frac{\partial I}{\partial r}=-C^{\prime} \frac{\partial V}{\partial t}=-\frac{2 \epsilon_{0} \pi r}{h} \frac{\partial V}{\partial t}
\end{gathered}
$$

where $V$ is the voltage, $I$ is the current, $L^{\prime}$ and $C^{\prime}$ are the inductance and capacitance per unit length of the radial transmission line, $r$ is the radial position, $h$ is the gap height, and $\mu_{o}$ and $\varepsilon_{o}$ are the permeability and permittivity of free space. Von Neumann stability analysis indicates that the system of equations must be solved with a backwards differencing scheme to achieve numerical stability, resulting in the matrix equation

$$
\boldsymbol{A} \phi_{n+1}=\phi_{n}
$$

where $\phi_{n}$ is a vector storing all of the nondimensionalized voltages and currents at timestep $n$ and $\mathbf{A}$ is a matrix representing the discretized telegrapher's equations, Kirchoff's laws, and the relations

$$
\begin{gathered}
V_{n+1}=\frac{L\left(I_{n+1}-I_{n}\right)}{\Delta t} \\
I_{n+1}=\frac{C\left(V_{n+1}-V_{n}\right)}{\Delta t} \\
V_{n+1}=R I_{n+1}
\end{gathered}
$$

at each inductor, capacitor, and resistor, respectively. The normalization constants used for nondimensionalization of the quantities in (4.3) are listed in Table 4.1, below. It can be easily shown these normalization constants are self-consistent; i.e. the normalization constant for capacitance divided by the normalization constant for length gives the normalization constant for 
capacitance per unit length. Scales were chosen for distance and voltage to match the conditions of the problem, and physical constants were used to fix all others.

Table 4.1: Physical scales used as normalization constants for the MAIZE circuit model

\begin{tabular}{|c|c|c|c|}
\hline Quantity & Symbol & Normalization & Value \\
\hline Velocity & $v_{S}$ & Speed of light & $2.998 \times 10^{8} \mathrm{~m} / \mathrm{s}$ \\
\hline Length & $x_{S}$ & Inner radius of transmission line & $0.345 \mathrm{~m}$ \\
\hline Time & $t_{s}$ & $x_{s} / v_{S}$ & $1.15 \times 10^{-9} \mathrm{~s}$ \\
\hline Voltage & $V_{S}$ & Total charging voltage on capacitors & $140 \mathrm{kV}$ \\
\hline Resistance & $R_{S}$ & Characteristic impedance of free space & $377 \Omega$ \\
\hline Inductance & $L_{S}$ & $R_{S} * t_{s}$ & $4.36 \times 10^{-7} \mathrm{H}$ \\
\hline Capacitance & $C_{S}$ & $t_{s} / R_{S}$ & $3.05 \times 10^{-12} \mathrm{~F}$ \\
\hline Current & $I_{S}$ & $V_{S} / R_{S}$ & $371 \mathrm{~A}$ \\
\hline Power & $P_{S}$ & $V_{S} * I_{S}$ & $6.00 \times 10^{7} \mathrm{~W}$ \\
\hline Energy & $E_{S}$ & $P_{S} * t_{s}$ & $0.0598 \mathrm{~J}$ \\
\hline $\begin{array}{c}\text { Inductance per } \\
\text { unit length }\end{array}$ & $L_{S}^{\prime}$ & Permeability of free space & $1.257 \times 10^{-6} \mathrm{H} / \mathrm{m}$ \\
\hline $\begin{array}{c}\text { Capacitance } \\
\text { per unit length }\end{array}$ & $C_{S}^{\prime}$ & Permittivity of free space & $8.854 \times 10^{-12} \mathrm{~F} / \mathrm{m}$ \\
\hline
\end{tabular}

\subsubsection{Static Load Parameters}

The simplest application of the model is the case when the load impedance and inductance are constant (or time-averaged to a constant value), and load voltage and current are calculated as functions of time. Figure 4.3 shows a current trace generated by the circuit model along with a current trace measured from the B-dot sensors using a static resistive load with known resistance of $80 \mathrm{~m} \Omega$ and inductance of $12 \mathrm{nH}$. The measurement demonstrates agreement well within the nominal $2.5 \%$ error on the B-dot probes. The disagreement late in time is attributed to a spurious signal acquired by the B-dot sensors that is dramatically larger on most dynamic load shots. This spurious signal always occurs well past peak current (>350 ns) and is attributed to a combination of charge buildup on the surface of the B-dot sensors and loss of magnetic insulation in the MITL after the voltage has changed signs. 


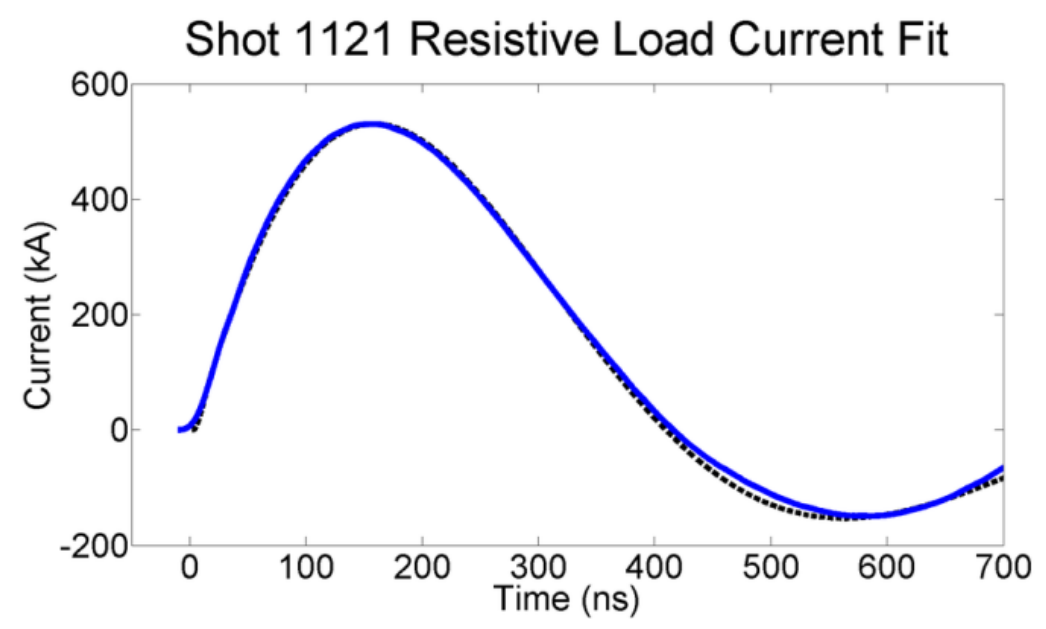

Figure 4.3: Comparison of measured current (solid curve) and simulated current (dashed curve) for a static load. The prediction agrees nearly exactly with the measurement until around $350 \mathrm{~ns}$ (well after peak current), after which point charge buildup on the surface of the B-dot sensors impacts their accuracy.

A parameter sweep of simulated peak current and risetime as functions of time-averaged load resistance and inductance is shown in Figure 4.4. For these simulations, the number of discrete transmission line elements $N$ was set to 15 , where each element represents a radius change, $d r$, of $3 \mathrm{~cm}$ with a transit time of $0.1 \mathrm{~ns}$. With the radial transmission line gap height of $1.3 \mathrm{~cm}$, impedances of the transmission line elements ranged from $0.96 \Omega$ on the (large-radius) generator side to $2.32 \Omega$ on the (small-radius) load side. Increasing $N$ to values higher than 15 did not produce measurable differences in load peak current or risetime for a variety of test cases. The load inductances used on Figure 4.4 include the inductance of adapting hardware that connects the load to the radial transmission line.

Because the peak current and risetime contours on Figure 4.4 are nearly orthogonal, the time-averaged resistance and inductance for a given shot can be determined by finding the intersection of experimentally measured peak current and risetime. This procedure was performed for a sampling of previous MAIZE runs including planar foil [49], cylindrical liner [37, 43], short-circuit, and resistive load shots, as indicated on the plots. For the dynamic load 
(planar foil and cylindrical liner) shots, the current pulses predicted from the combination of time-averaged inductance and resistance usually matched the observed current pulses to within a few percent, indicating time-dependent behavior could be approximated as perturbations on the average parameters. The initial inductances for these loads were $8 \mathrm{nH}$ (planar foil), $23 \mathrm{nH}$ (cylindrical liner), $19 \mathrm{nH}$ (short-circuit), and $12 \mathrm{nH}$ (resistive load). These inductances generally agree with the time-averaged inductances observed on Figure 4.4; the larger variation in observed inductance on the short circuit load shots is attributed to arcing observed in the load region. While the time-averaged resistance of the dynamic loads exhibits more shot-to-shot variation; on average it is roughly half the pre-shot resistances $(150 \mathrm{~m} \Omega$ for planar foils and 50 $\mathrm{m} \Omega$ for cylindrical liners).

The inductance-resistance parameter space for the double planar wire array (DPWA) loads used in the shots discussed in Section 4.4 and presented in References [47] and [52] is highlighted on the plot. The initial inductance of the DPWA loads, including the hardware adapting to the radial transmission line, was calculated to be $18 \mathrm{nH}$ using ANSYS Maxwell. The inductance space includes an estimated uncertainty of $2 \mathrm{nH}$ from the Maxwell simulation as well as the observed increases in inductance on DPWA shots (described in section 4.4) due to pinching. Time-averaged resistances were experimentally observed to range from approximately $50 \mathrm{~m} \Omega$ to $70 \mathrm{~m} \Omega$ for these shots. 


\section{Peak Current +/- 70 kV Charge}

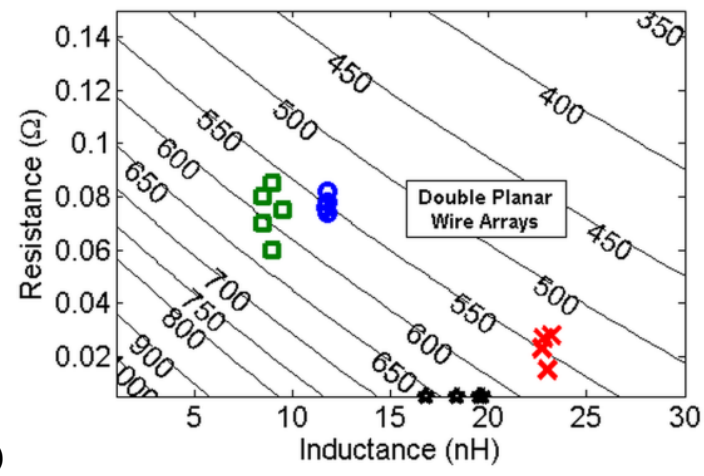

Risetime +/- 70 kV Charge

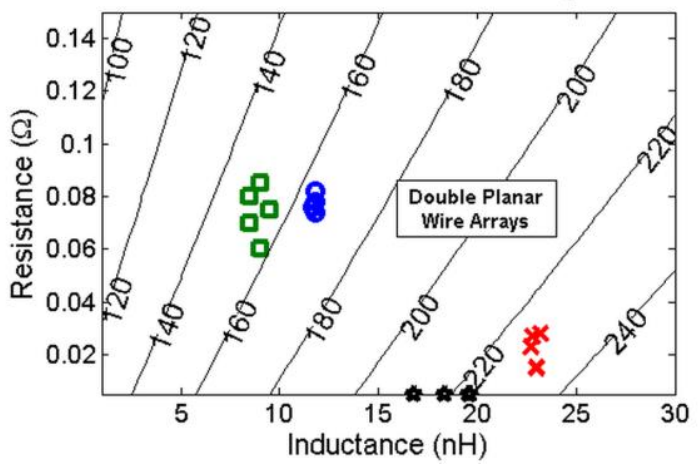

(b)

Figure 4.4: Results from load parameter sweep showing (a) peak current in kiloamps and (b) risetime in nanoseconds at the load. Observed parameters from a sample of MAIZE shots are indicated as follows: Planar foil [49] (green square) cylindrical liner [37, 43] (red X); static resistive load (blue circle); short circuit load (black star). The rectangular region denotes the approximate parameter space spanned by the DPWA shots; the relatively large parameter space is due to shot-to-shot variation in wire dynamics as well as in-shot inductance changes due to pinching.

It is noteworthy that peak current and risetime values for the DPWA shots on MAIZE presented in Refs [47] and [52] do not, in general, fit within the parameter space outlined on Figure 4.4. This discrepancy is due to an abnormally high firing delay in a large number of switches. On some shots, late firing switches extended the risetime and lowered the peak current; on others, arcing in the transmission line occurred, which artificially lowered the risetime observed by B-dot current monitors. These processes are described in detail in Section 4.3.

\subsubsection{Dynamic Load Inductance}

A measurement of time-dependent load inductance on Z-pinches of wire arrays, gas puffs, or cylindrical liners provides useful insight on timing of mass redistribution during the pinch process and can also be used to calculate an effective current-carrying radius of the imploding plasma. Previous measurements of inductance obtained from simultaneous voltage and current measurements on wire array implosions on $\mathrm{Z}$ [53] were able to obtain information on the timing of wire core motion and effective radius. Additionally, voltage and current measurements of gas puff Z-pinches on Saturn were used to calculate time-dependent inductance 
and energy coupled into the load, assuming negligible load resistance $[54,55]$. These techniques assume that load inductance is dominant over resistance, and compute inductance from

$$
L_{\text {load }}(t)=\frac{V(t)}{d I / d t}
$$

Measuring voltage on an LTD can prove difficult because the inductive isolation of the outer case from the transmission line often leads to ground loops when voltage divider probes are employed, and attempts to field differential D-dot probes [40] were complicated by the presence of electron flow in the MITL. Implementing the circuit model described in the previous section, using the charging voltage as an initial condition, enables a measurement of load inductance requiring only a current measurement, eliminating the need to measure voltage. To accomplish this, the measured current is replaced by the nondimensionalized load inductance in the vector $\phi$ of (4.3), and the matrix A becomes time-dependent to represent the inclusion of known, timedependent current. Solving (4.3) then gives the voltage and current at all other points in the system along with the time-dependent $L_{\text {Load. }}$ This method is also valid if a nonzero timeaveraged resistance $R_{\text {Load }}$ is included, provided changes in load impedance are dominated by changes in inductance. The $2.5 \%$ error in current measurement specifies the smallest inductance change that can be measured; for a load inductance of $20 \mathrm{nH}$, this minimum measurable change is less than $1 \mathrm{nH}$. 


\subsection{Circuit Model with Variable Switch Timings}

In normal LTD operation, all spark-gap switches fire simultaneously to within their nominal jitter (usually less than $10 \mathrm{~ns}$, which does not measurably affect the output current pulse). However, the DPWA experiments described in Section 4.4 were complicated by the fact that the LTD was nearing the end of a rebuild cycle, leading to many of the switches firing late by 10s or even 100s of ns. A diagnostic consisting of optical fibers connected to an array of photomultiplier tubes (PMTs) was fielded to measure switch firing times, with each optical fiber aimed at the trigger plane of a single switch. Up to 7 switches could be monitored on a single shot. Output signals from the photomultiplier tubes for a properly functioning switch and a poorly functioning switch are shown in Figure 4.5. The initial pulse occurs when the trigger gap breaks down, and the large spike in signal coincides with the switch becoming fully conductive, consistent with previous observations on these switches [48]. Each switch was monitored for five shots immediately after the DPWA campaign, giving a total of 200 switch timing measurements that were used to establish a model of switch firing timings. It was found that of the 40 switches, on each shot $14 \pm 4$ switches fired within $30 \mathrm{~ns}$ of the start of current, $8 \pm 2$ fired during the risetime of the current pulse, and $18 \pm 6$ fired after peak current had occurred. This diagnostic was also fielded on 4 random switches for each shot during the campaign to observe overall trends in switch behavior on the dynamic loads. The spread in switch firing times introduced arcing in the transmission line and also had dramatic effects on both the peak current and the shape of the current pulse. For a self-magnetically insulated radial transmission line with $r>h$, where $r$ is a radial position and $h$ is the gap height, the magnetic cutoff condition can be approximated as 


$$
I_{\text {cutoff }}(r)>\frac{2 \pi r}{\mu_{0}} \sqrt{\frac{2 m_{e} V(r)}{e h^{2}}}
$$

where $I_{\text {cutoff }}$ is the minimum current required for magnetic insulation, $V$ is the voltage at a given radial position, and $m_{e}, \mu_{0}$, and $e$ are the mass of an electron, permeability of free space, and fundamental charge respectively. If fewer switches fire on an LTD, the magnitude of the voltage pulse (and, consequently, $I_{\text {cutoff }}$ ) is mostly unaffected, but the observed current is lower, causing the outer portion of the radial transmission line (where $r$ and $I_{\text {cutoff }}$ are largest) to lose magnetic insulation and become vulnerable to arcing. Since the switch firing times were observed to be highly variable on the DPWA experiments, a few shots had enough switches firing on time or during the risetime of the pulse to establish magnetic insulation, while other shots lost significant load current to arcs. Visible inspection of the transmission line confirmed that arcs occurred at a large radius that was outside the radius of the B-dot probe array. This allowed the presence or absence of arcs on a particular shot to be detected by the B-dots; on shots with arcing one or two of the B-dots showed a sharp drop in current early in the pulse, whereas on shots with no arcing all four B-dots integrated to give the same pulse shape (although the magnitude was often different, due to the nonuniform current distribution caused by the high variation in switch firing). 

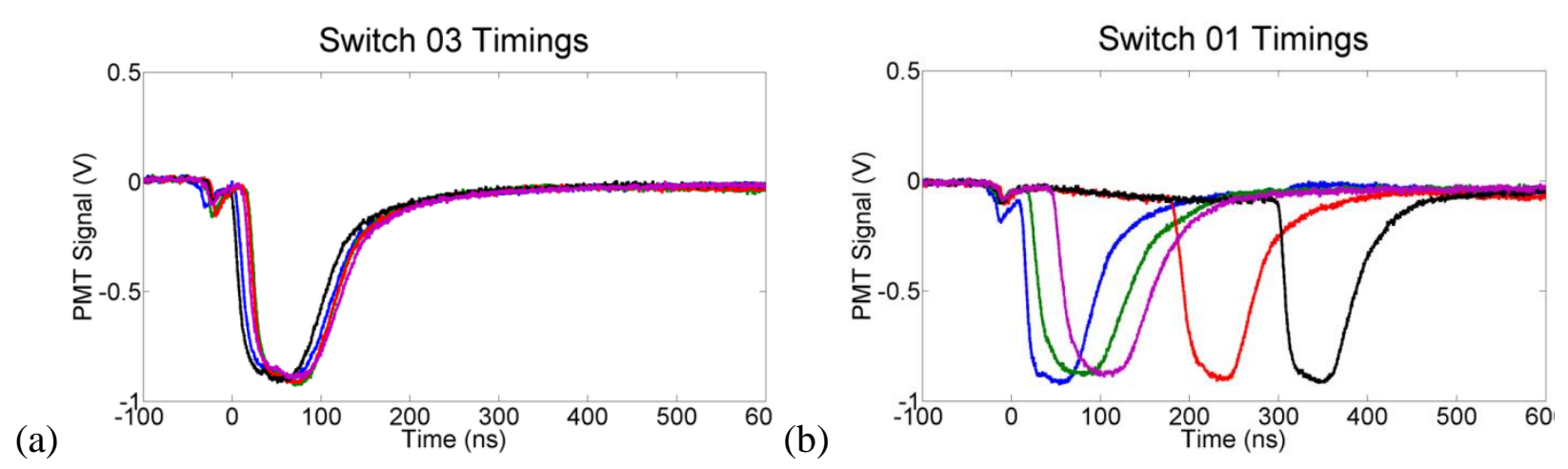

Figure 4.5: Comparison of switch behavior over 5 consecutive shots into a static resistive load for (a) a properly functioning switch with approximately $10 \mathrm{~ns}$ jitter and (b) a malfunctioning switch with closing times ranging from $10 \mathrm{~ns}$ to $300 \mathrm{~ns}$ after nominal start of current.

Shots with arcing are virtually impossible to model from a circuit standpoint, as little information is available about the location, size, and conductivity of breakdown paths in the transmission line. DPWA shots that exhibited significant pinching on x-ray diagnostics were also the shots that did not arc in the transmission line, which is expected as these shots had the highest current delivered to the load. Because the model implementation described in Section 4.2 assumes simultaneous switch firing, an additional circuit model was developed in LTSPICE [56] treating each brick independently to account for the effects of switch firing time delays; this model was applied to several arc-free shots to determine time-dependent inductance. In this model, the inductance and resistance of each brick are set to 40 times the lumped inductance and resistance of the generator section and the capacitance of each brick is set to $1 / 40^{\text {th }}$ of the lumped capacitance represented in Figure 4.2. Switch trigger times are roughly estimated from the PMT measurements described above. Time-averaged load resistance and fine adjustments to the trigger times are determined by matching the measured current pulse to the simulated current pulse early in time (up to approximately $150 \mathrm{~ns}$ after start of current, since the earliest observed pinch occurred after $200 \mathrm{~ns}$ ) and late in time (at approximately $350 \mathrm{~ns}$, just before spurious B-dot 
signals are expected). The initial load inductance was calculated using ANSYS Maxwell and estimated assuming self-inductance dominates for the 12 parallel, $5.1 \mu \mathrm{m}$ diameter, $0.9 \mathrm{~cm}$ long wires; both approaches gave $1.4 \mathrm{nH}$.

Time-dependent inductance of pinching DPWA loads is calculated by comparing measured current with output from the model using the initial load inductance. Because pinches and corresponding inductance changes were observed to occur near peak current on a timescale that is fast compared to the pulse length, the inductance change as a function of time is approximated by holding constant the stored energy $1 / 2 L I^{2}$, where $L$ represents the total inductance of the machine. Therefore, the difference between measured current and predicted current corresponds to a change in inductance.

Typical rates of inductance change during a pinch were of the order 0.1 to $0.2 \mathrm{nH} / \mathrm{ns}$. This rapid change generates a voltage pulse that propagates along the transmission line back to the switches. Near peak current, this voltage pulse is given by

$$
V_{\text {inductive }}(t)=L_{\text {load }}(t) \frac{d I}{d t}+I(t) \frac{d L_{\text {load }}}{d t} \approx I(t) \frac{d L_{\text {load }}}{d t}
$$

where $V_{\text {inductive }}$ is the inductive voltage drop across the load and $I$ is the current through the load. At peak currents near $400 \mathrm{kA}$ the magnitude of this pulse often exceeded $50 \mathrm{kV}$. When this voltage pulse reached the switches, the untriggered switches immediately fired, sending a secondary current pulse into the load region. This explanation is supported by the PMT traces observed on shots with a successful pinch; switches that did not fire early in the current pulse all fired nearly simultaneously at a time corresponding to x-ray diode signals. The switch firing behavior, coupled with the natural load dependence of LTDs, is believed to be the reason for the 
long risetimes and low peak currents observed on MAIZE compared to previous DPWA experiments [57-59].

\subsection{Inductance Measurements on Planar Wire Array Implosions}

\subsubsection{Planar Wire Array Setup and Diagnostics}

The experimental configuration used in the DPWA implosions is shown in Figure 4.6. For these shots, the loads consisted of two sets of six $5.1 \mu \mathrm{m}$ stainless steel 304 wires spanning an AK gap of $9 \mathrm{~mm}$ with total load mass of $19.4 \mu \mathrm{g}$. The distance between wires in the same plane, referred to as the interwire spacing, was $1 \mathrm{~mm}$, and the distance between wire planes, referred to as the intergap spacing, was varied between $3 \mathrm{~mm}$ and $6 \mathrm{~mm}$. Figure 4.7 shows a closeup view of a DPWA load. This load region was connected to the radial MITL by a coaxial adapter hardware section that replaced the triplate transmission line used in previous experiments $[37,49]$. The total inductance of the load region including this adapter was calculated from a magnetostatic simulation performed in ANSYS Maxwell and was found to be approximately 18 $\mathrm{nH}$. 


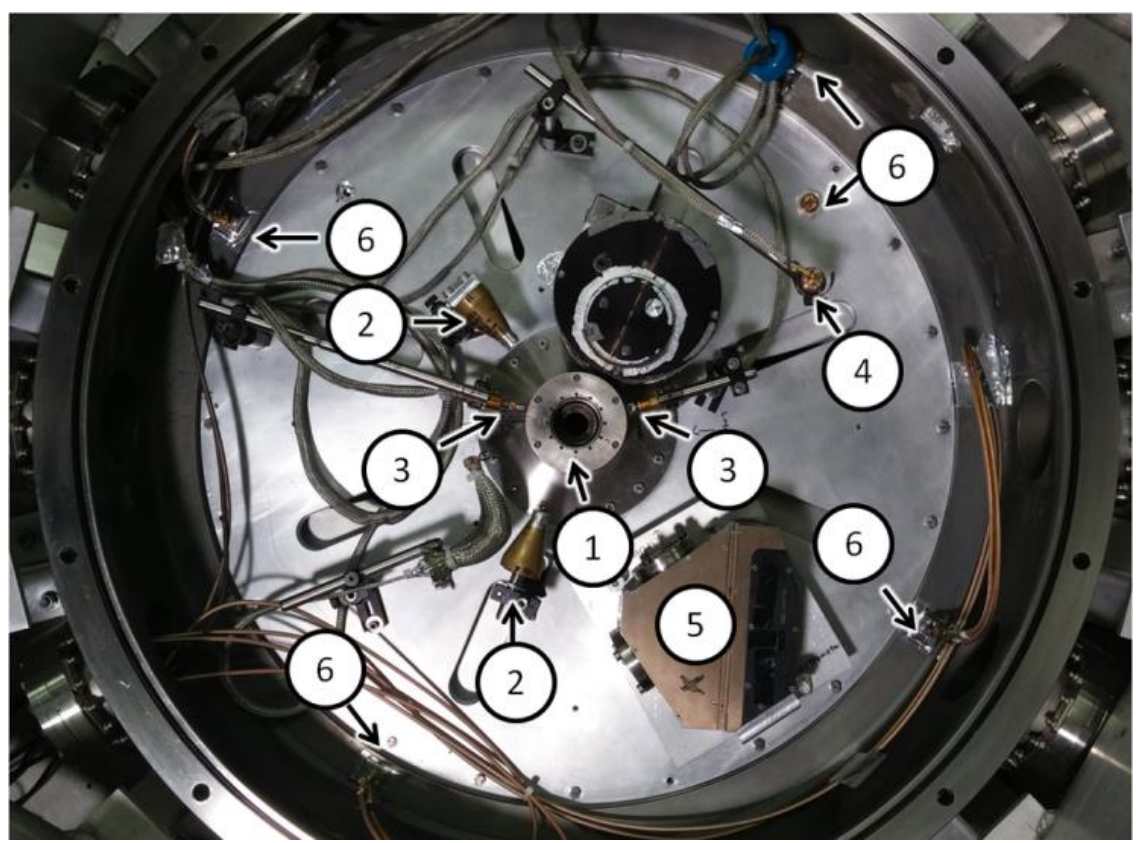

Figure 4.6: MAIZE vacuum chamber top down view with load hardware and diagnostics. (1) Load hardware adapter; (2) Pinhole camera with x-ray film; (3) X-ray diode; (4) Faraday Cup (in loading position); (5) KAP x-ray spectrometer; (6) Differential B-dot current probe

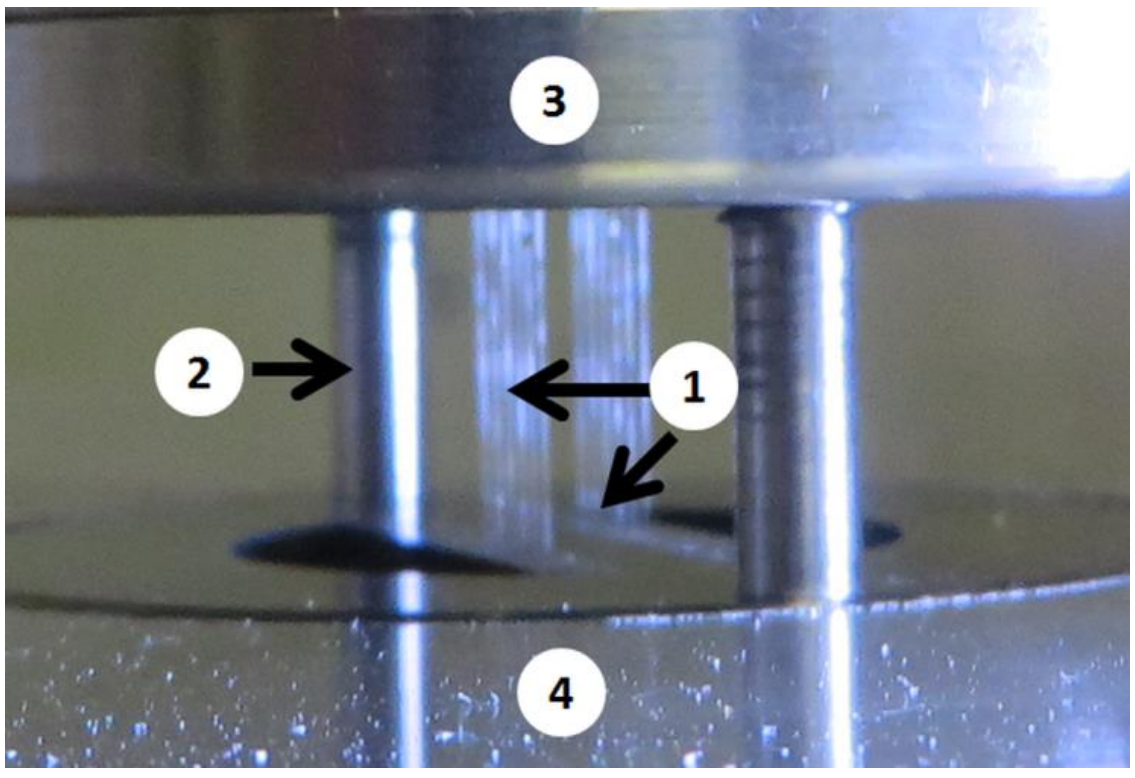

Figure 4.7: Close up isometric view of load region for DPWA experiment. (1) Planar array conisisting of six 5.1 $\mu \mathrm{m}$-diameter 304 SS wires; (2) Anode return current post; (3) Cathode; (4) Anode 
The 12-frame self-emission/shadowgraphy diagnostic described in Chapter 3 was unavailable for the DPWA campaign. Instead, the $532 \mathrm{~nm}, 2 \mathrm{~ns}$, frequency-doubled Nd:YAG pulse was split into four beams with approximately 1.3 degrees of angular separation between adjacent beampaths to obtain four-frame shadowgraphy on four Canon Rebel digital cameras with approximately $10 \mathrm{~ns}$ between frames. Time-dependent X-ray emission was monitored by three AXUV-HS-5 x-ray diodes (0.7 ns time resolution) with filters of cutoff energies $1.4 \mathrm{keV}$, $3.5 \mathrm{keV}$, and $9 \mathrm{keV}$. Additional diagnostics fielded in these experiments, which were part of the second collaborative UNR-UM campaign, are described in Ref 7.

\subsubsection{Calculated Inductance Results}

Figure 4.8 shows current traces from the two shots with the most significant $x$-ray production of the campaign. Shot 937, shown in Figure 4.8a, had an initial interplanar spacing (the distance between the two planes of wires) of $3 \mathrm{~mm}$, and shot 938, shown in Figure 4.8b, had an initial interplanar spacing of $6 \mathrm{~mm}$. The measured current is plotted along with predicted current from the circuit model described in section IV using the $1.4 \mathrm{nH}$ inductance of the initial solid-wire DPWA. Time-averaged resistance was found to be $65 \mathrm{~m} \Omega$ for shot 937 and $50 \mathrm{~m} \Omega$ for shot 938. Signals from $\mathrm{x}$-ray diodes with filters at $3.5 \mathrm{keV}$ and $9 \mathrm{keV}$ are shown on the same plot to give information on timings of the pinches (the $1.4 \mathrm{keV}$ diode signals, not shown, made a prompt jump to saturation on both shots at approximately $220 \mathrm{~ns}$ and remained at the saturation value for several hundred ns). Timings from the four-frame shadowgraphy system are also indicated on the plots. These shadowgraphs are shown in Figure 4.9. For shot 937, the timing of the last shadowgraph occurs approximately at the peak of the pinch; for shot 938, the timing of the shadowgraphs lines up such that the latest image approximately captures the start of x-ray emission. From Figure 4.9b, it is clear that a significant amount of mass still exists at the initial 
wire locations as late as $215 \mathrm{~ns}$ on the 6-mm gap DPWA, but between the $215 \mathrm{~ns}$ and $225 \mathrm{~ns}$ frames much of this mass has transported to the central plasma. The last frame was captured approximately $50 \mathrm{~ns}$ prior to peak x-ray emission, as evident on Figure $4.8 \mathrm{~b}$. The images from figure $4.9 \mathrm{a}$ were captured during the pinch and show only a central plasma column. The qualitatively different implosion dynamics between the two shots are explained by the variation in aspect ratio, which is the ratio of the width of one of the wire planes to the intergap spacing. Shot 937 had a relatively high aspect ratio of 1.67, which produces an implosion that is characterized by the formation of a single precursor region, while shot 938 had a lower aspect ratio of 0.83 , which produces an implosion that is characterized by both precursor formation and independent implosions of material from the two wire planes.
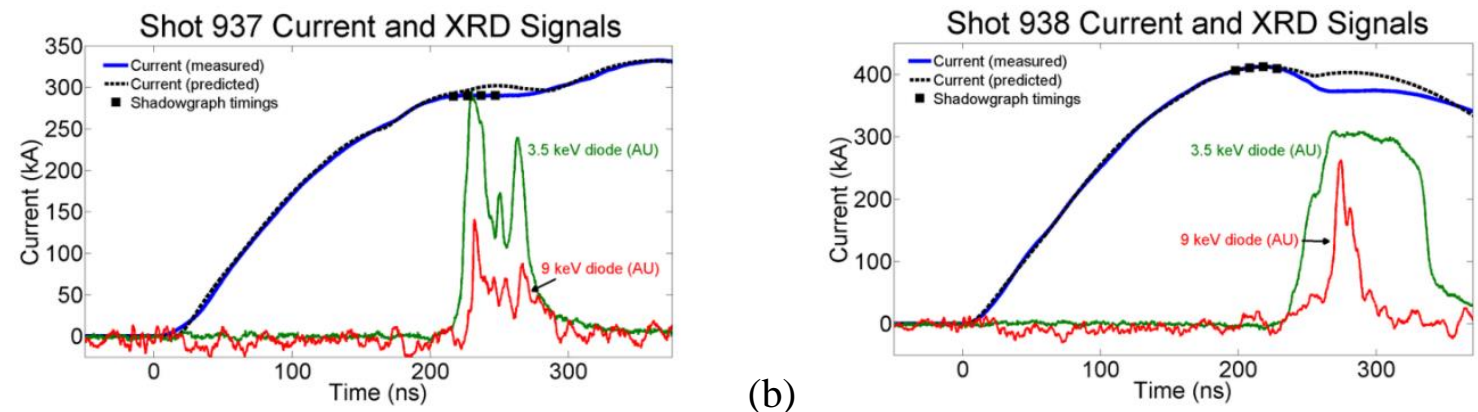

Figure 4.8: Predicted and measured current traces from (A) shot 937 and (B) shot 938. Laser shadowgraph timings and signals from the $\mathrm{x}$-ray diodes with filters of $3.5 \mathrm{keV}$ and $9 \mathrm{keV}$ in arbitrary units are also shown. 


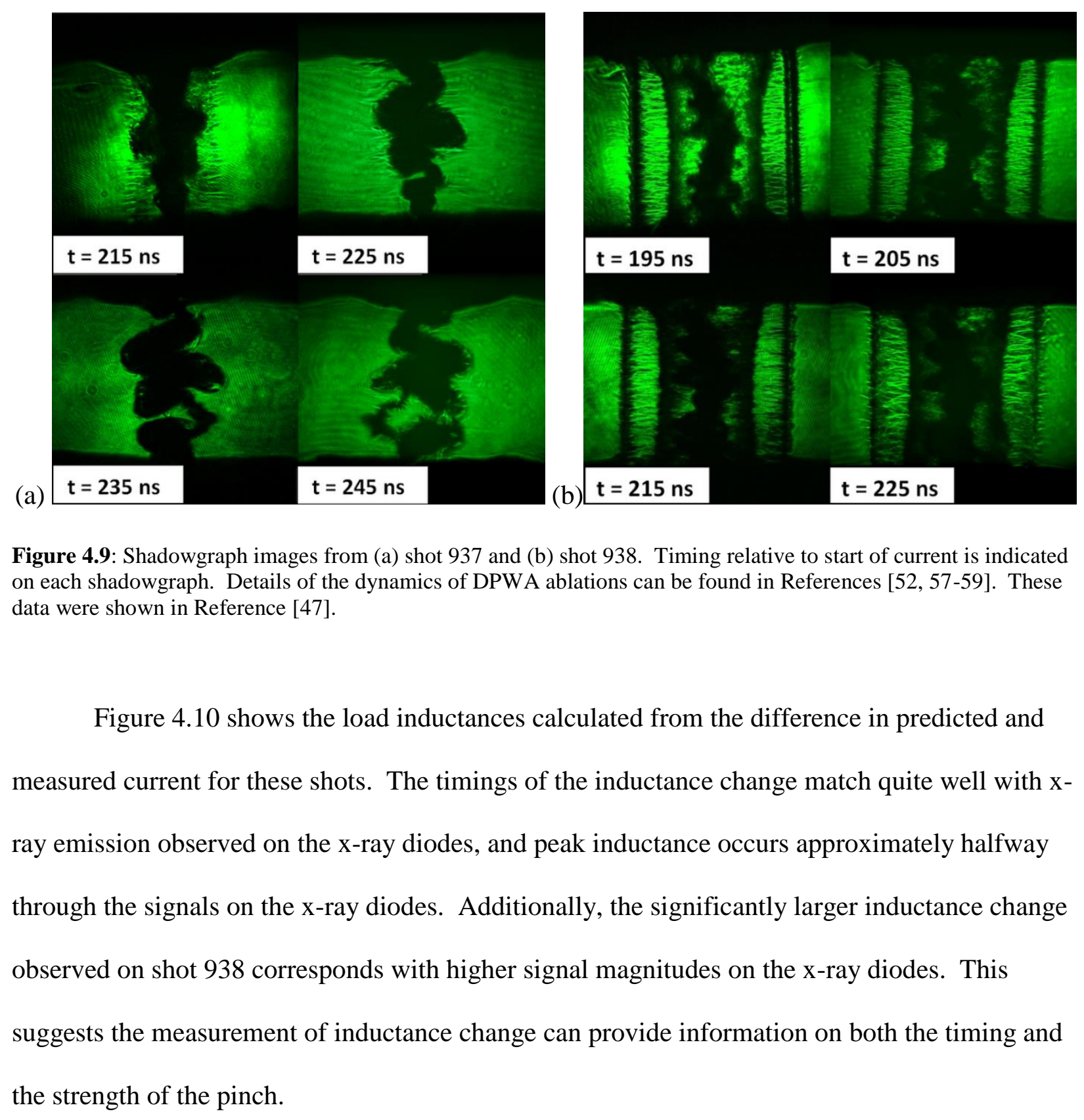



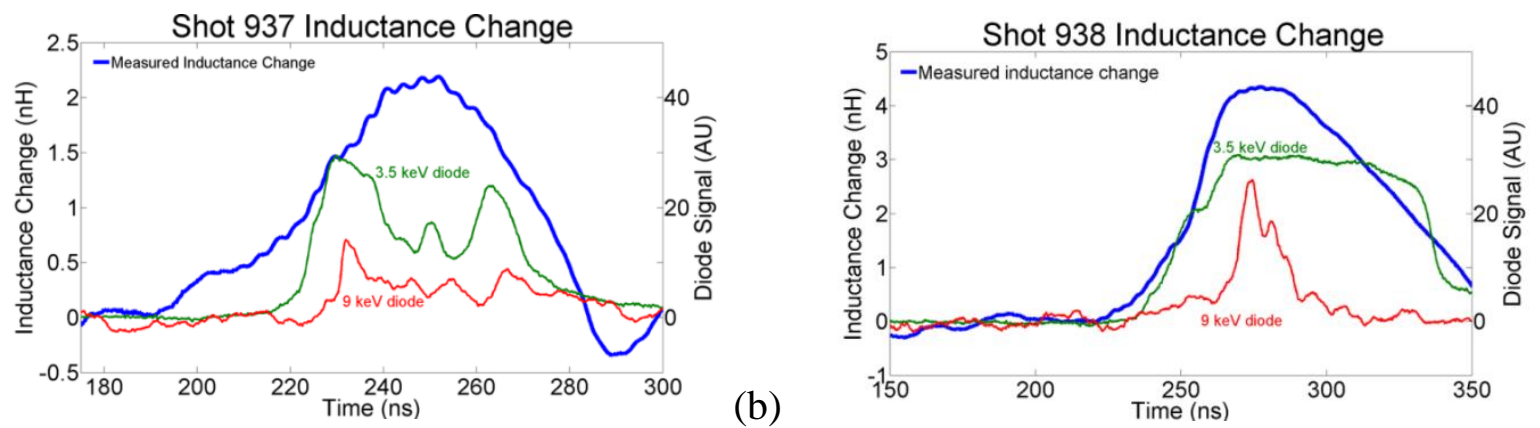

Figure 4.10: Inductance change as a function of time for (a) shot 937 and (b) shot 938 (blue curves). Signals from the $3.5 \mathrm{keV}$ (green) and $9 \mathrm{keV}$ (red) $\mathrm{x}$-ray are also shown. The timings of the inductance spikes agree very well with measurements of $\mathrm{x}$-ray emission.

A minimum effective current-carrying diameter was determined from the magnitude of the inductance change for both shots, assuming the self-inductance of the load region dominates over the mutual inductance with the return current posts. Via this calculation, the diameter was found to be $3.0 \mathrm{~mm}$ for shot 937 and $1.0 \mathrm{~mm}$ for shot 938 . Because the shadowgraphs from shot 938 captured an early time with considerable mass still at the initial wire positions, they did not provide an adequate comparison point to an estimate of a single pinch column, but fortunately the shadowgraphs from shot 937 occurred while the pinch was in process. Figure 4.11 shows a close-up of the latest available shadowgraph from shot 937, which occurred within 5 ns of peak inductance. The $3.0 \mathrm{~mm}$ effective diameter is also shown on this figure, corroborating the inductance change-based estimate. While the dramatic kink and sausage instability structure complicates the approximation of a single effective radius of current, the diameter of the central plasma column is on the order of the estimated current carrying diameter, indicating that the measurement is at least reasonable. 


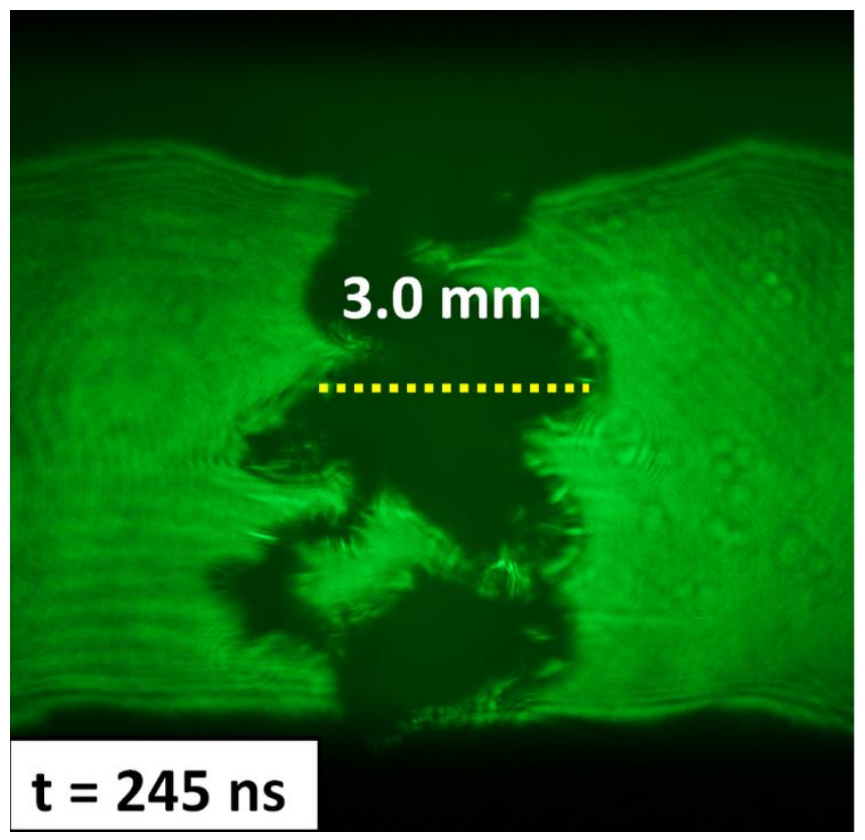

Figure 4.11: Shadowgraph taken at approximate time of peak emission from shot 937. Effective current carrying radius calculated from the inductance change is also shown. Initial interplanar wire gap spacing was $3 \mathrm{~mm}$.

\subsection{Inductance Measurements on Cylindrical Liner Implosions}

To provide a better evaluation of the ability of this circuit analysis method to measure average current-carrying radius, the method was applied to current measurements from cylindrical liner implosions [37] performed on MAIZE. Loads for these experiments were the $400 \mathrm{~nm}, 99.1 \%$ pure aluminum foils wrapped in liner geometry around the dumbbell supports described in Chapter 3. These experiments utilized the 12-frame self-emission/shadowgraphy diagnostic to provide up to 12 images per shot. As with the DPWA experiments, time-dependent inductance of the load is calculated from the difference between measured current and predicted current and is used to determine current-carrying radius. The initial self-inductance of the aluminum liner for this shot was $2.4 \mathrm{nH}$, and the total load-side inductance including the adapter hardware for this load was found to be $23 \mathrm{nH}$ using Maxwell. Time-averaged resistance was found to be $21 \mathrm{~m} \Omega$ using Figure 4.4 for the observed peak current of $550 \mathrm{kA}$ at $230 \mathrm{~ns}$. Figure 
4.12 shows the predicted and measured current for this shot. The calculated effective currentcarrying diameter is shown on a series of framing camera images from a single shot on Figure 4.13 and the observed column diameter from each image is plotted against the time-dependent load inductance and calculated current-carrying diameter on Figure 4.14. Because the plasmavacuum interface is unstable to the sausage, kink, and magneto Rayleigh-Taylor instabilities, the experimental plasma column diameter shown on Figure 4.14 represents a weighted average position of the interface, and the high and low error bars represent the plasma column at its thickest and thinnest points, respectively.

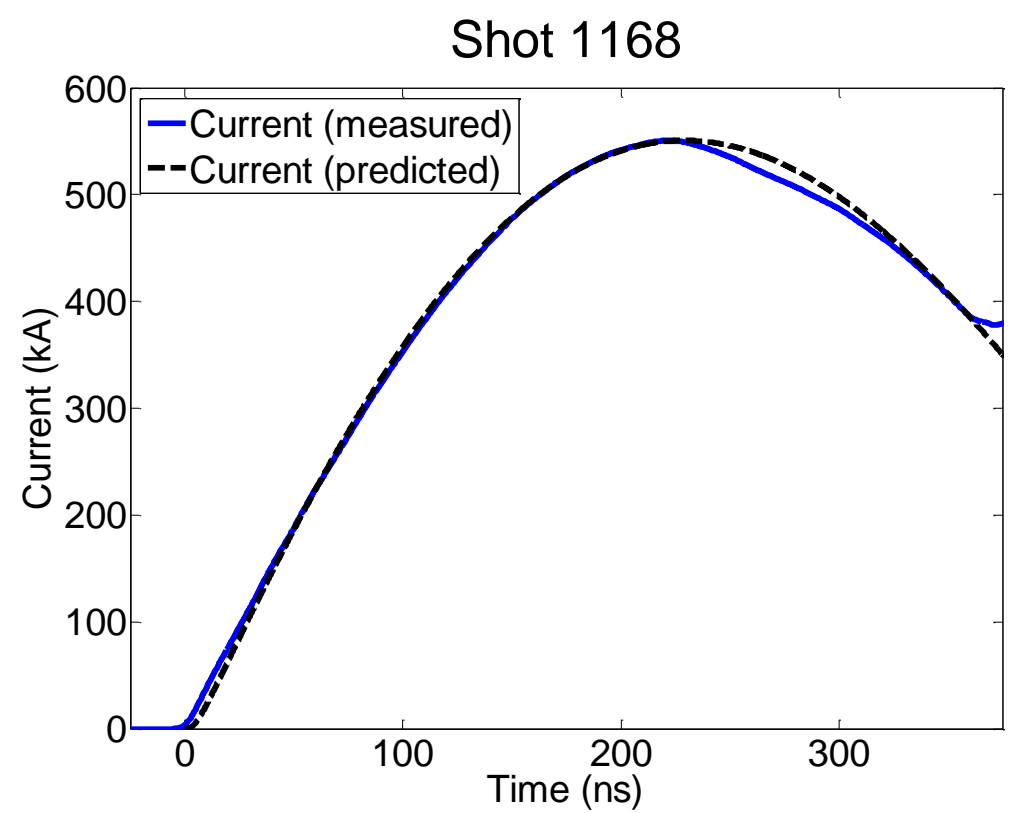

Figure 4.12: Predicted and measured current traces for shot 1168, an implosion of an initially solid $400 \mathrm{~nm}$ aluminum liner. 

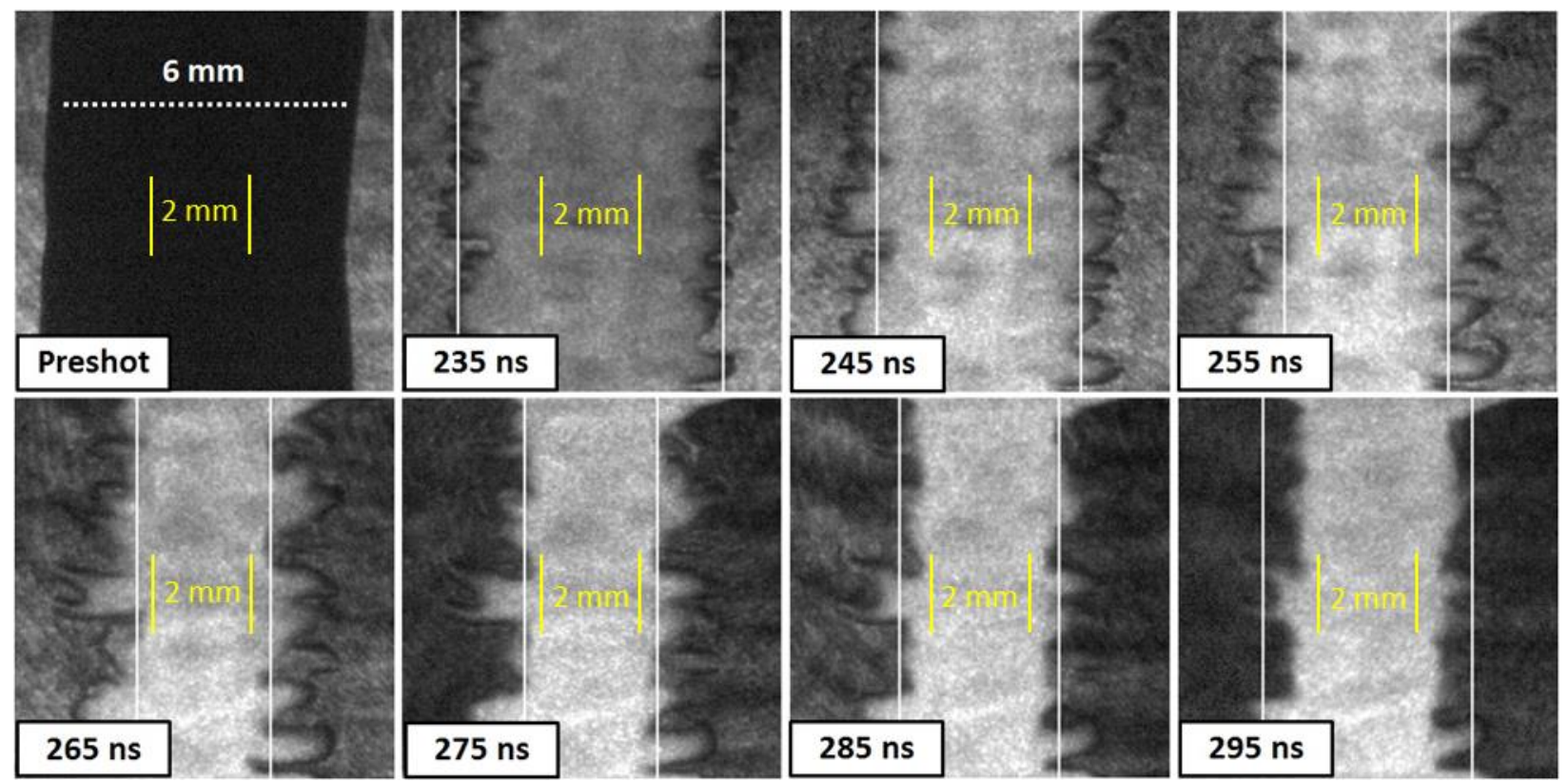

Figure 4.13: Self-emission/shadowgraph images with effective current-carrying radius indicated in white vertical bars. The first image shows a pre-shot shadowgraph for establishing spatial scale. The central column of the plastic support is approximately $2 \mathrm{~mm}$ in diameter, indicated by the yellow vertical bars.

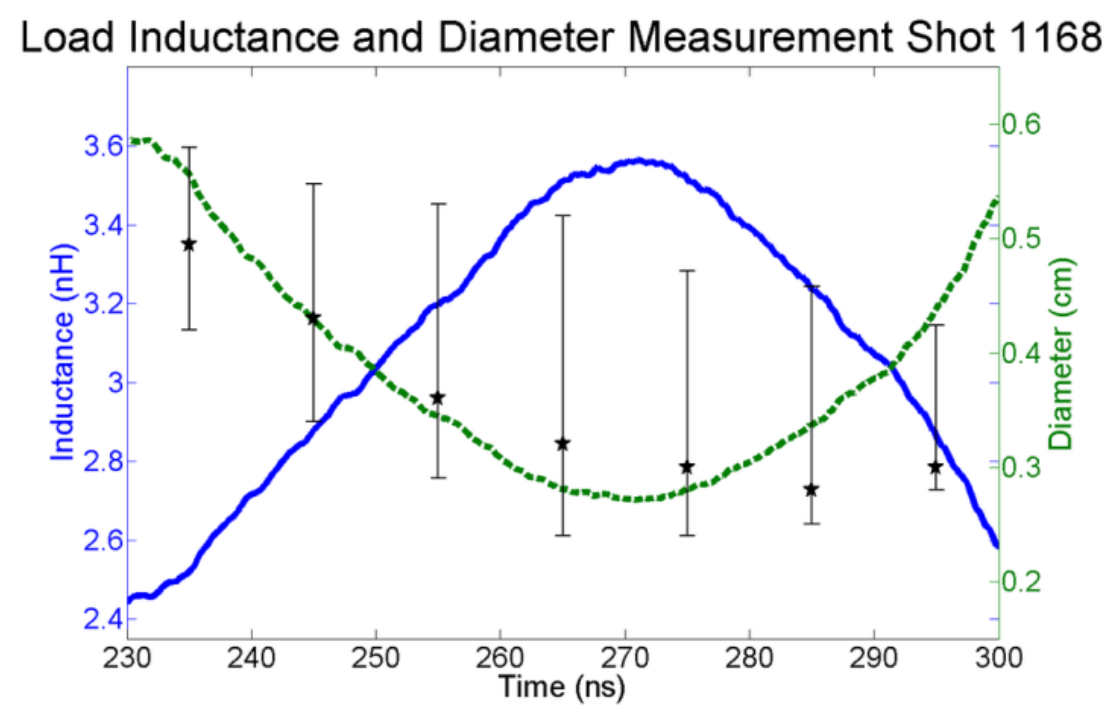

Figure 4.14: Plasma column diameter measured from 10 ns-spaced framing camera images (stars) plotted against calculated current-carrying diameter (dashed curve) and load inductance (solid curve). Agreement between experiment and prediction is observed for all shadowgraph frames except the final frame at 295 ns. The error bars represent absolute upper and lower bounds on the plasma/vacuum interface diameter given by the plasma column at its thickest and thinnest point, respectively. 
Figures 4.13 and 4.14 demonstrate remarkable agreement between the time-dependent calculated current-carrying diameter and the diameter of the plasma column visible on the shadowgraphs for all times except the latest available shadowgraph at $t=295 \mathrm{~ns}$. The disagreement on the last frame is likely due to the assumption that plasma resistance is constant, which may be violated late in time for a sufficiently strong pinch. The compression of the plasma column is expected to increase the temperature, which lowers the Spitzer-like plasma resistivity. If this decrease in resistance is non-negligible, it would lead to an underestimate of the inductance late in time, which explains the overestimate of current-carrying radius observed in the last frame. Self-emission observed on the CCD images supports this explanation; average CCD response measured in the plasma region on the $295 \mathrm{~ns}$ frame is approximately $15 \%$ higher than the response in the plasma region on the $235 \mathrm{~ns}$ frame. 


\section{CHAPTER 5}

\section{Results from Electrothermal Instability Experiments}

This chapter presents results from planar foil ablations with the objective of observing and measuring the growth of stratified structures. While recent studies have observed striations as mass perturbations on pulsed power explosions of wires [60] and planar foils [61], and experiments at Sandia National Labs have observed temperature perturbations as striations on ablating cylindrical rods [62], the results presented here demonstrate the first time-resolved measurements of temperature perturbations that grow perpendicular to current on foil ablations. It will be shown that these temperature perturbations agree with both qualitative and quantitative predictions of ETI theory.

\subsection{Current, voltage, and resistance measurements}

The voltage and current monitors are the primary source of information about the energy delivered to the foil and, therefore, its phase and average temperature. Typical voltage/current data are shown in Figure 5.1 for ablation of a 400 nm-thick Al foil. Figure 5.1 shows several features that are present on all foil ablations: a sudden change in $d I / d t$ that corresponds to the foil melting, a simultaneous dramatic increase in voltage across the load, and a sudden drop in voltage occurring 50 to $100 \mathrm{~ns}$ later. The voltage increase is due to the increase in resistance of the foil as it transitions through the liquid phase and begins to vaporize, and the subsequent drop results from ionization of aluminum vapor and the creation of parallel conductive plasma channels. The voltage across the foil is effectively zero at times before and after this peak 
because the resistance of the load is sufficiently low that the inductance of the circuit dominates the total impedance.

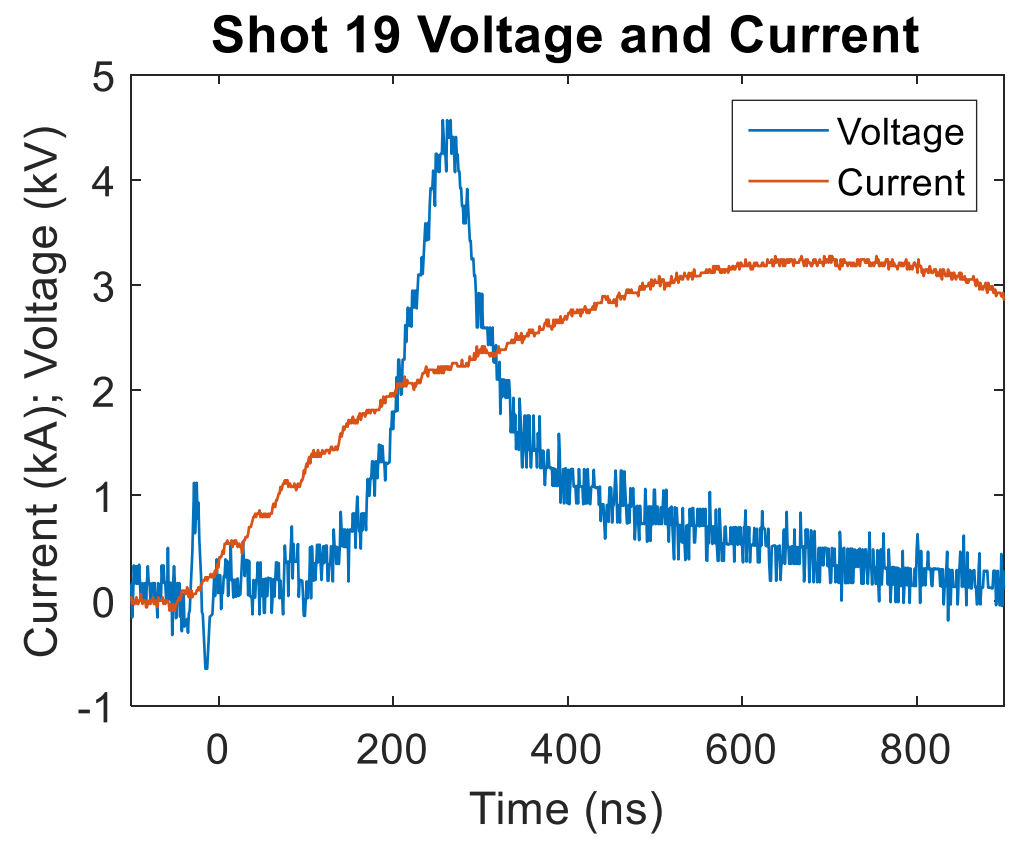

Figure 5.1: Typical voltage and current plot. The voltage spikes as the resistance of the load increases sharply through the liquid and vapor phases, then drops as the vapor transitions to partially ionized plasma. For this shot, the foil dimensions were $400 \mathrm{~nm} \times 1.0 \mathrm{~cm} \times 0.9 \mathrm{~cm}$.

Because the inductance of the $0.9 \mathrm{~cm}$-long sections of foil is minimal (under $2 \mathrm{nH}$ for the narrowest foil strips used in these experiments), the voltage and current measurements were used to determine the resistance of the load as a function of time. This resistance was used, along with the initial dimensions of the load, to calculate the average foil resistivity. Because the initial dimensions (rather than the expanded foil dimensions due to heating of the foil) are used, comparisons to tabulated resistivity values in this work use the modified resistivity described in Equation (2.38c*), and unless otherwise specified the term "resistivity" refers to resistivity calculated from initial foil dimensions. Figure 5.2 shows resistivity determined from the voltage and current measurements of Figure 5.1. Additionally, resistivity calculated from load enthalpy 
is shown on Figure 5.2 as a dashed curve, where the energy deposited to the load is determined from

$$
H_{\text {load }}(t)=\int_{o}^{t} V\left(t^{\prime}\right) I\left(t^{\prime}\right) d t^{\prime}
$$

Resistivity is calculated from (2.38) up to the point at which the foil reaches the vaporization temperature.

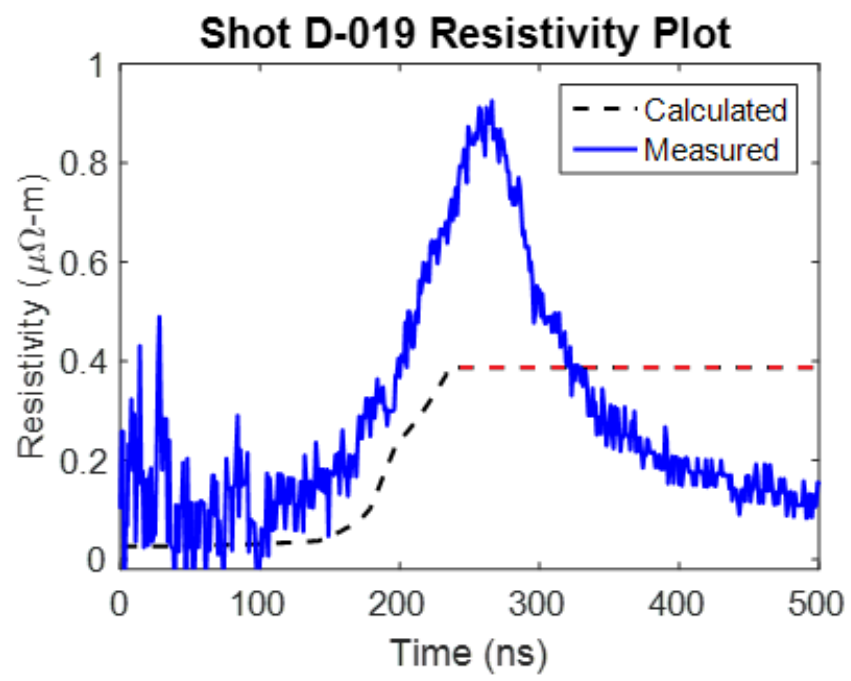

Figure 5.2: Measured foil resistivity, averaged over the foil and assuming the initial dimensions of the foil (solid blue curve); resistivity calculated from enthalpy delivered to the load is also plotted (dashed black curve) until the foil reaches the vaporization temperature. After this time, the resistivity of molten aluminum at the vaporization temperature is shown (dashed red line) as a reference.

While the resistivity of biphase liquid/vapor aluminum is more difficult to determine, the resistivity of liquid aluminum at the vaporization temperature, $\eta_{v}$, is shown as a dashed red line on Figure 5.2 to draw attention to several important points. First, the measured resistivity begins to overshoot the calculated resistivity between $150 \mathrm{~ns}$ and $200 \mathrm{~ns}$ and far overshoots the value of $\eta_{v}$ at the peak occurring around $260 \mathrm{~ns}$. High values of peak resistivity were consistently observed; for most shots the peak resistivity was around 2 to 3 times greater than $\eta_{v}$, and on a few shots the peak was even higher. This indicates that sections of the foil have resistivity (and 
therefore temperature) higher than the bulk foil resistivity, and that some sections of the foil are at least partially entering the vapor phase, which has higher resistivity than liquid aluminum. It is unlikely that the surrounding gas has ionized by this point in the foil ablation, because parallel conducting plasma channels would be expected to lower the overall measured resistance. Additionally, the average resistivity is lower than $\eta_{v}$ late in time. For that to be the case, either the entire foil has transitioned into the higher conductivity plasma state, or parallel plasma channels have formed at the previously high-resistivity locations. The first possibility is ruled out in Figure 5.3, which shows the load enthalpy (expressed as a change from room temperature conditions) plotted against the resistivity curve shown in Figure 5.2. On Figure 5.3, key points of predicted phase changes are highlighted in vertical dashed lines indicating the enthalpy required to raise the foil to the melting temperature (black), complete the melting process (blue), raise the foil to the vapor temperature (green), and complete the vaporization process (red).

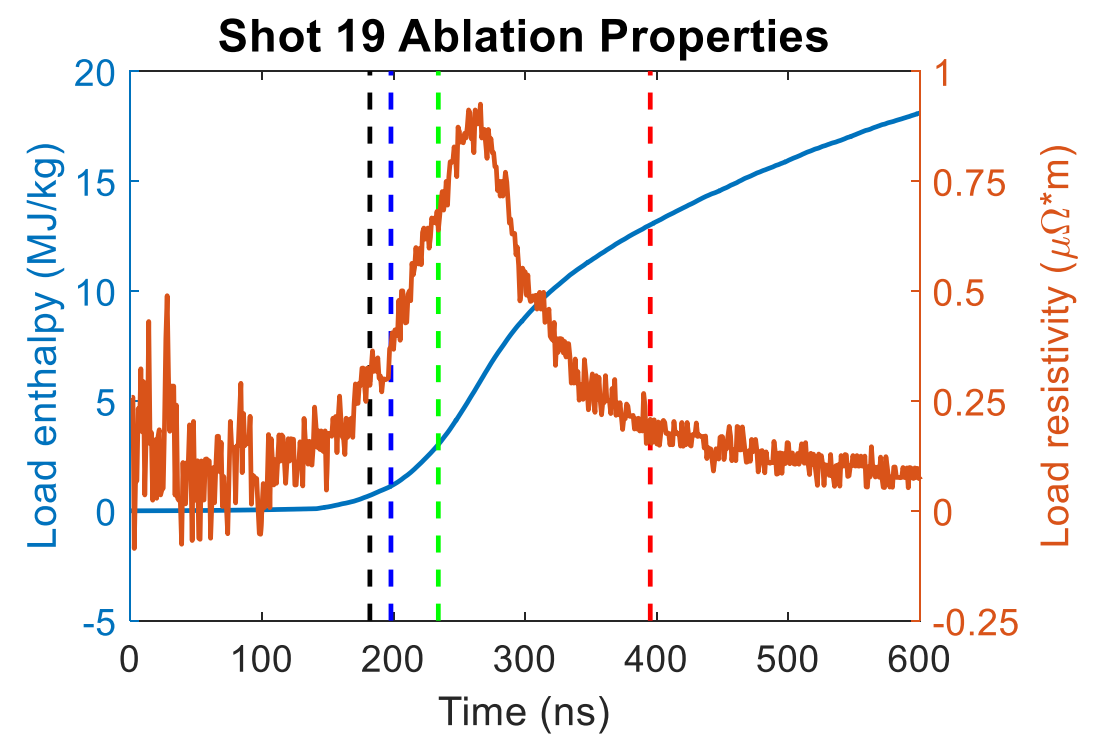

Figure 5.3: Measured foil-averaged resistivity (orange curve) plotted against enthalpy delivered to the load (blue curve). Points of phase transition are shown with dashed vertical lines; these represent the onset of melting (black), the completion of melting (blue), the onset of vaporization (green), and the completion of vaporization (red), all assuming uniform energy deposition. 
From Figure 5.3, even if the energy deposition were perfectly uniform, the bulk foil does not absorb sufficient energy to complete vaporization, let alone ionization, by the time the resistivity rises above $\eta_{v}$. The interpretation of the electrical data is therefore as follows: sections of the foil increase in temperature (and therefore resistivity) faster than the bulk material, increasing the average resistivity of the foil over the resistivity of a uniformly heated foil. These hotter sections undergo vaporization and continue to increase in temperature until the resistive voltage drop across them is sufficient to cause breakdown, lowering their resistivity below $\eta_{v}$. This process occurs before the bulk foil absorbssufficient energy to complete the process of vaporization. Optical emission data presented in the following section supports this understanding of the ablation process.

\section{2: Visible Self-Emission Measurements}

Figure 5.4 shows a 12 -frame sequence of self-emission from the ultrafast imaging system described in Section 3.2.1. These images show three phases that are present on most foil ablations. First, striations of hot, partially ionized material form perpendicular to the direction of current flow and increase in length. Later in time, around 320 to 325 ns on Figure 5.4, adjacent striations begin to merge into longer wavelength structures. Eventually, this merging creates plasma channels across large sections of the foil surface; these channels develop bright and dark filamentations in the parallel-to-current direction. 

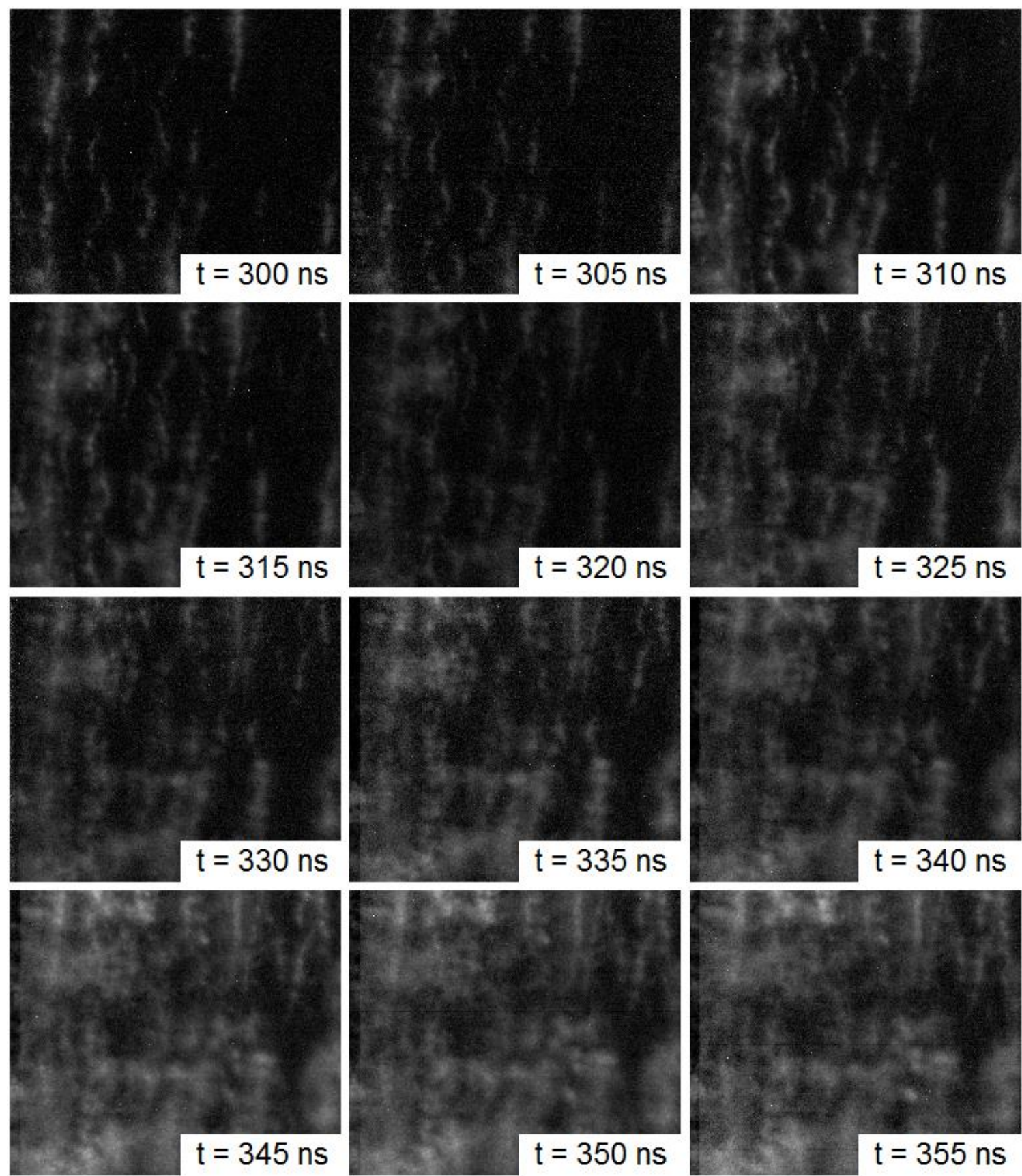

Figure 5.4: Typical image sequence of $5 \mathrm{~ns}$-exposure images showing self-emission striations on an $800 \mathrm{~nm} \times 0.7$ $\mathrm{cm} \times 0.9 \mathrm{~cm}$ foil ablation. Imaging window is $1.25 \mathrm{~mm} \times 1.08 \mathrm{~mm}$ with $2 \mu \mathrm{m}$ resolution. This sequence shows the stages of striation formation, merging, and eventual formation of filamentary plasma channels. Current flows from left to right.

The timing of the formation of these bright structures relative to the resistivity measurements described in Section 5.1 is presented in Figure 5.5. Shots D-16 and D-19 were 
taken in an air background, shots D-18 and D-20 were taken in an SF 6 background, and shot D17 was taken in an argon background. Two shots each in air and $\mathrm{SF}_{6}$ are shown to represent the shot-to-shot variation on foils with identical initial conditions. Peak bulk resistivity tends to range between $0.6 \mu \Omega-\mathrm{m}$ and $1.2 \mu \Omega-\mathrm{m}$, and light emission, as measured by the PMT, begins during the resistivity rise, with variability in time of around $50 \mathrm{~ns}$. 


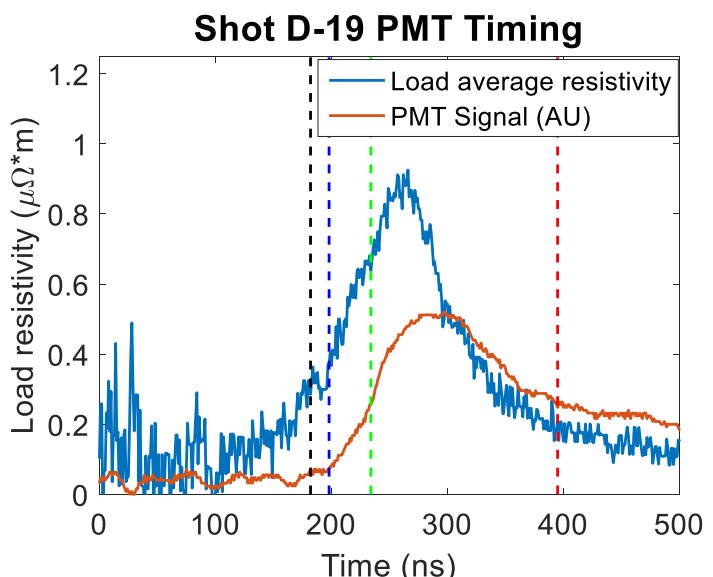

(a)

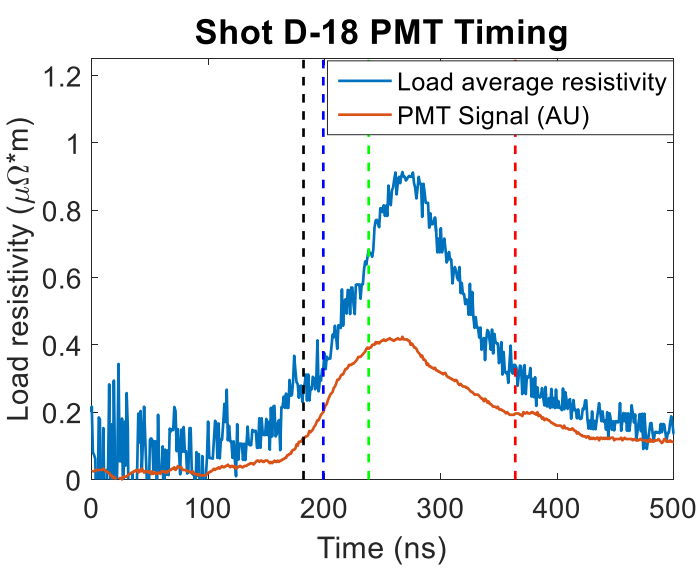

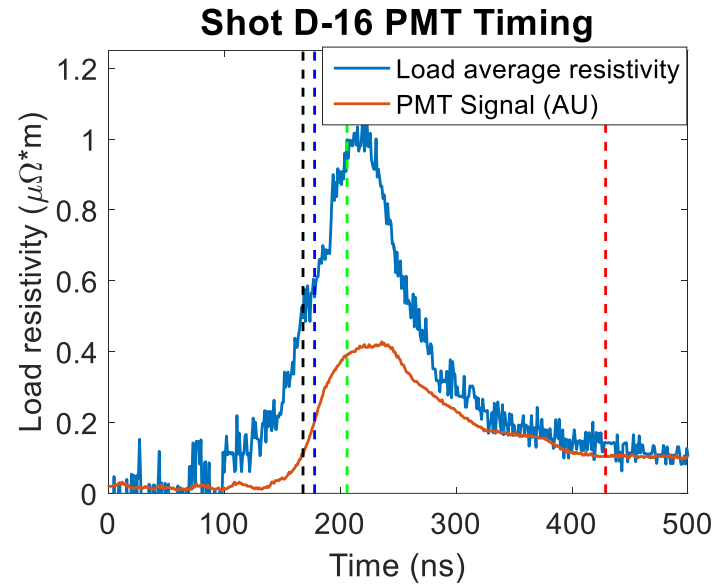

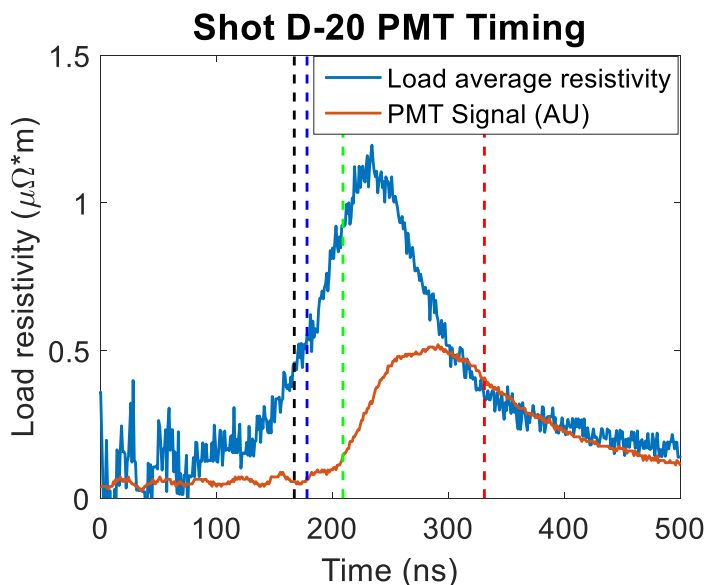

(d)

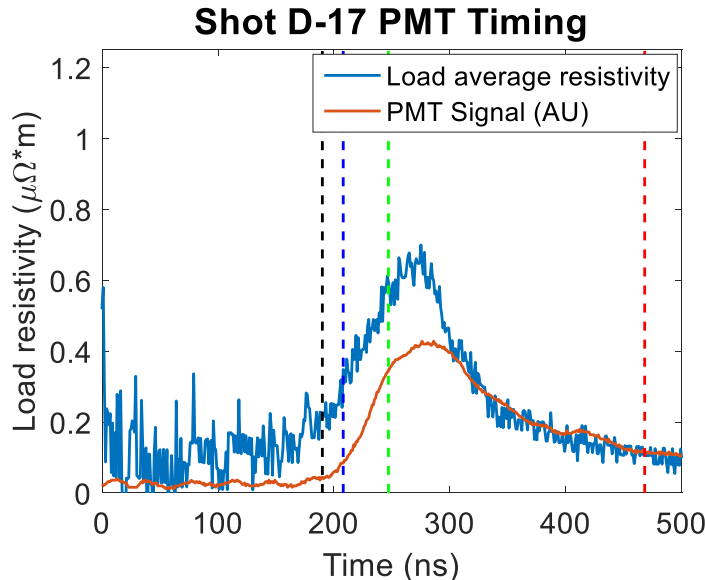

(e)

Time (ns)

Figure 5.5: Plots of PMT signal and average foil resistivity for $400 \mathrm{~nm}$ x $1.0 \mathrm{~cm}$ x $0.9 \mathrm{~cm}$ aluminum foil ablations. The same phase transition timings shown in Figure 5.3 are included. Background gases were (a) and (b) air, (c) and (d) $\mathrm{SF}_{6}$, and (e) argon, all at atmospheric pressure. 
The PMT signals on Figure 5.5 occur at similar times relative to the resistivity peaks compared to striation formation observed on framing camera shots. A total of five PMT shots were performed in air, with an additional three in argon and three in $\mathrm{SF}_{6}$. Significant shot-toshot variation in light emission timing was observed among shots in the same gas, although light emission consistently begins during the risetime of the resistivity spike. This shot-to-shot variation within gases is believed to be due to random variations in foil initial resistivity conditions causing ETI to develop faster on some shots than others. No additional significant variation in light emission timing was observed between the three gases. Because the gases have different dielectric strengths - particularly $\mathrm{SF}_{6}$, which is often used in high voltage insulation applications - this indicates that aluminum vapor, rather than the background gas, is predominantly breaking down to form the plasma seen on Figure 5.4. By the time the resistivity has risen to drive the $\mathrm{kV}$-scale voltage spike, sections of the foil have vaporized, and the aluminum vapor is easier to break down than the surrounding gas. This observation is supported by data from optical emission spectroscopy, shown in Figure 5.6. 


\subsubsection{Optical Spectroscopy}

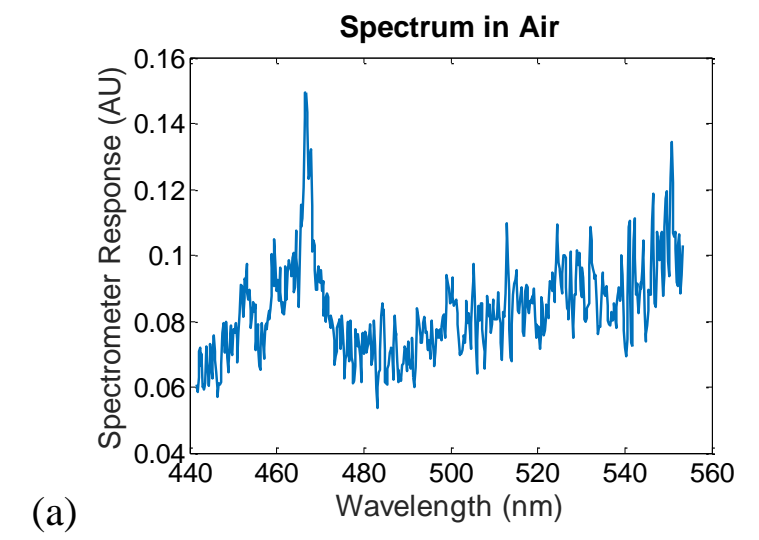

(b)
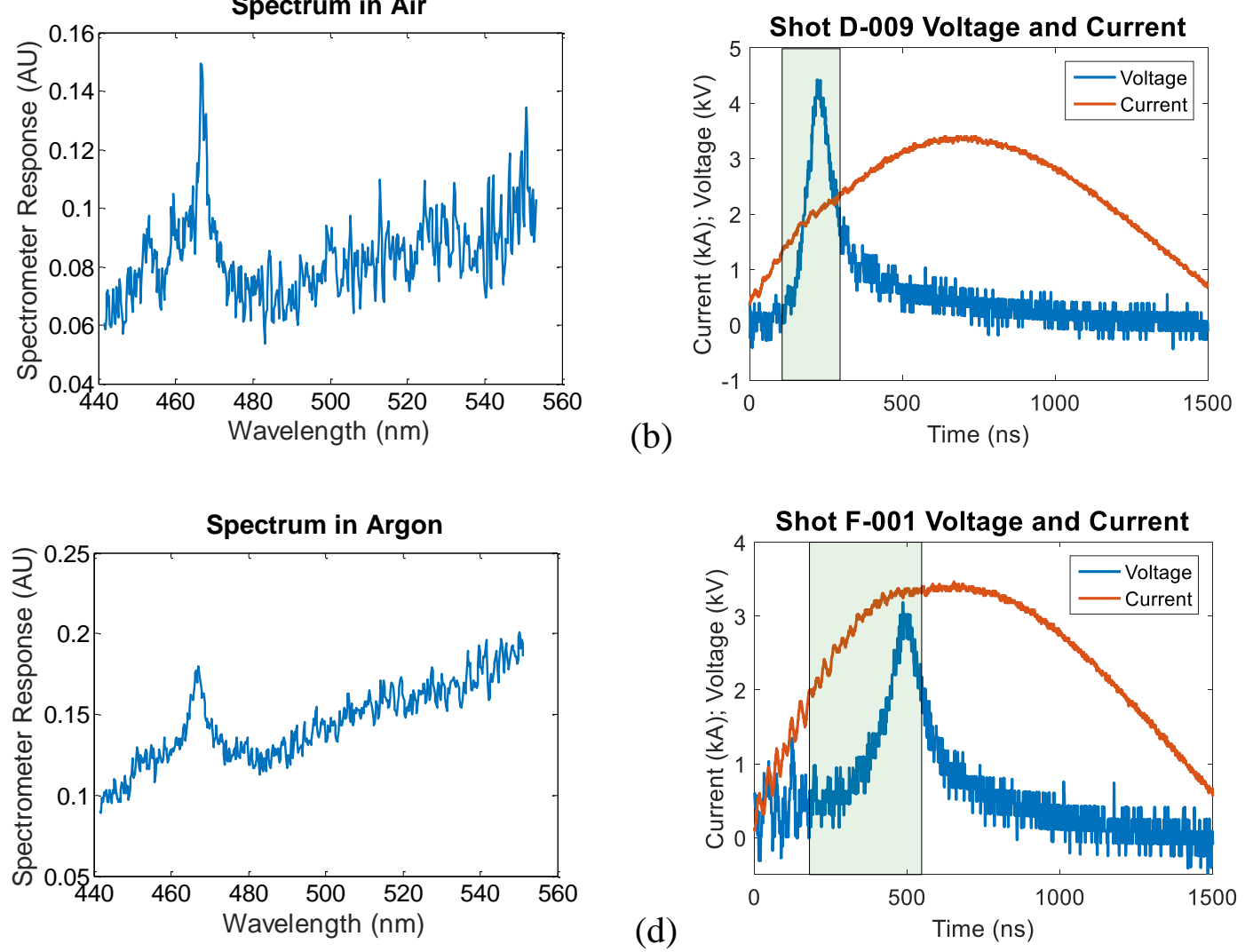

(d)

PrismSPECT Simulation, $1.0 \mathrm{eV}, 1 \mathrm{e} 18 \mathrm{~cm}^{-3}$

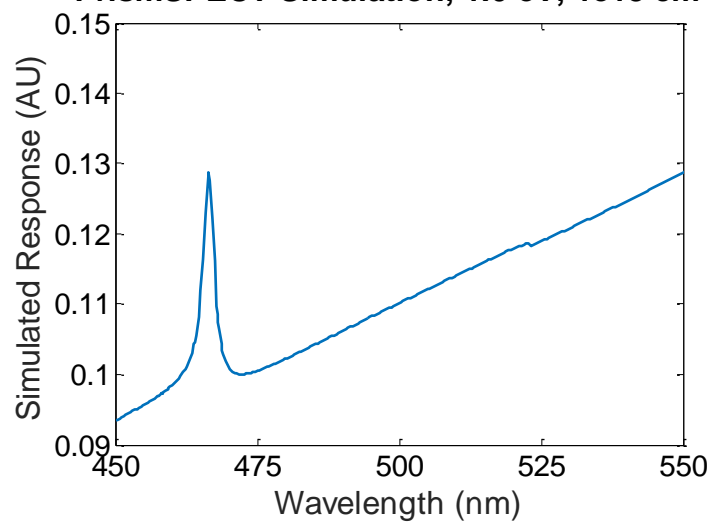

Fig. 5.6: Time- and spatially-integrated spectral measurements of foil self-emission and electrical signals from ablation of (a, b) $400 \mathrm{~nm} \times 1.0 \mathrm{~cm} \times 0.9 \mathrm{~cm}$ foil and (c, d) an $800 \mathrm{~nm} \times 0.7 \mathrm{~cm} \times 0.9 \mathrm{~cm}$ foil. The background gas for (a) was atmospheric air, and the background gas for (c) was atmospheric pressure argon. The upward slope of the continuum is believed to be due to blackbody emission (due to an average surface temperature that changes as a function of time). Plots (b) and (d) show the corresponding voltage and current plots with the ICCD gate width shown as green boxes. Plot (e) shows a non-local thermodynamic equilibrium (NLTE) PrismSPECT simulation (provided by Dr. Sonal Patel of Sandia National Labs) for aluminium (neutral and singly ionized) in the same wavelength range. 
Figure 5.6 shows two experimentally observed spectra collected in air and argon backgrounds, time-integrated over the window during which instability features develop. The same line at approximately $466 \mathrm{~nm}$ is visible on both the air-background and argon-background spectra. Light levels were lower on the argon spectrum because the light had to be transmitted through the clear plastic bag used to house the argon fill; a wider slit width had to be employed on the spectrometer to account for the lower light levels, causing the broadened line observed on Figure 6c. This line at $466 \mathrm{~nm}$ appears on an NLTE simulation of neutral and singly ionized aluminum performed in PrismSPECT [63] by Dr. Sonal Patel of Sandia National Laboratories [64]. Spectra taken at similar times compared to Figure 5.6a in the windows of 400 to $450 \mathrm{~nm}$, 550 to $650 \mathrm{~nm}$, and 600 to $700 \mathrm{~nm}$ showed no additional significant lines (no light was observed below $400 \mathrm{~nm}$ because the attenuation of the plastic fiber optic is prohibitively high at wavelengths below $400 \mathrm{~nm}$ ). Because the same dominant line appears in different fill gases and matches up with a simulated aluminum line, it is reasonable to conclude that the source of light emission is aluminum plasma. PrismSPECT simulations were performed for densities ranging over the $10^{16}$ to $10^{18} \mathrm{~cm}^{-3}$ scales and for temperatures from 0.5 to $1.5 \mathrm{eV}$. The simulation at $1 \mathrm{x}$ $10^{18} \mathrm{~cm}^{-3}$ and $1.0 \mathrm{eV}$ matches the experimental spectra quite well, so the electron density and temperature of striations (averaged over the gate window) is likely in these regimes.

\subsubsection{Sources of Light Emission}

The detector-counts under the line on Figure 5.6a account for roughly $2 \%$ of the total counts in the visible spectrum between $400 \mathrm{~nm}$ and $700 \mathrm{~nm}$. Therefore, the bright features on the self-emission framing camera diagnostic are assumed to be continuum dominated, rather than line-emission dominated. The continuum processes of Bremsstrahlung radiation and two-photon emission are discounted, as they are only relevant in high temperature $(>>1 \mathrm{eV})$ and hydrogen- 
or helium-like plasmas, respectively. This leaves radiative recombination and blackbody-like emission as potential sources of continuum light. Assuming no ionization past the first ionization state has occurred, the total power radiated from radiative recombination in a thin slab of width $w$, length $L$, and thickness $d$ is given by

$$
P_{r r}=K_{r r} n_{e} n_{i} w L d E_{i 1}
$$

where $K_{r r}$ is the radiative recombination reaction rate (a function of electron temperature), $n_{e}$ and $n_{i}$ are the electron and ion densities, and $E_{i l}$ is the first ionization energy. Values of $\mathrm{K}_{\mathrm{rr}}$ are taken from the FLYCHK data tables [65].

Total power emitted in the visible spectrum from blackbody radiation from the same slab (assuming an emissivity close to 1 ) is

$$
P_{b b}=S(T) w L
$$

where

$$
S(T)=\int_{\lambda_{1}}^{\lambda_{2}} S(\lambda) d \lambda=\int_{\lambda_{1}}^{\lambda_{2}} \frac{2 \pi h c^{2}}{\lambda^{5}} \frac{1}{\exp \left(\frac{h c}{\lambda k_{B} T}\right)-1} d \lambda
$$

In (5.3), $h$ is Planck's constant, $c$ is the speed of light in vacuum, $\lambda$ is the wavelength of emitted radiation, $k_{B}$ is Boltzmann's constant, and $T$ is the material temperature. The ratio of radiativerecombination to blackbody emission is then given by

$$
\zeta=\frac{P_{r r}}{P_{b b}}=\frac{K_{r r}\left(T_{e}\right) n_{e} n_{i} E_{i 1} d}{S(T)}
$$

where $T_{e}$ is the electron temperature.

Taking the conservative assumptions that all emission from radiative recombination occurs in the visible, $n_{e}=n_{i}=10^{18} \mathrm{~cm}^{-3}$ (from the PrismSPECT simulation in Figure 5.6e), $T=$ $4000 \mathrm{~K}$ (on the low end of temperatures observed in Section 5.3), and $T_{e}=1.0 \mathrm{eV}$, this ratio, $\zeta$, is approximately $2 \%$. Therefore, the dominant optical emission mechanism is expected to be 
blackbody-like emission, and the procedure detailed in Section 3.3 is employed to make the temporally and spatially resolved temperature measurements reported in Section 5.3.

The simulation generating the spectrum shown in Figure 5.6e calculates an ionization fraction of 78\%, or an ion-neutral ratio of 3.5. Simulations at lower electron temperatures (and therefore lower ionization fractions) showed the additional presence of aluminum I lines that were not observed in the experimental spectrum. This order unity ionization fraction is also predicted by the Saha ionization equation for reasonable material temperatures at the estimated $10^{18} \mathrm{~cm}^{-3}$ electron density. For plasmas dominated by neutral species and singly-ionized ions, the Saha equation is given by

$$
\frac{n_{I I}}{n_{I}}=\frac{2 Z_{I I}(T)}{n_{e} Z_{I}(T)}\left(\frac{2 \pi m_{e} k_{B} T}{h^{2}}\right)^{\frac{3}{2}} e^{-E_{i 1} / k_{B} T}
$$

where $n_{I}$ is the number density of neutral atoms, $n_{I I}$ is the number density of ions, $m_{e}$ is the mass of an electron, and $Z_{I}$ and $Z_{I I}$ are the partition functions for neutral atoms and ions. The partition function is

$$
Z(T)=g_{1}+\sum_{j=2}^{\infty} g_{j} e^{-E_{j} / k_{B} T} .
$$

where $g_{j}$ is the degeneracy of the jth energy state and $E_{j}$ is the energy of each state referenced to the ground state. For calculations using (5.5), the partition function ratio was calculated using the NIST data tables for the first 15 states of aluminum I and aluminum II. From equation (5.5), the ion-neutral ratio is of order unity, given electron densities in the $10^{17}$ to $10^{18} \mathrm{~cm}^{-3}$ range, for material temperatures around 6000 to $8000 \mathrm{~K}$. As will be shown in Section 5.3, these are typical measured temperatures of the hot striation material.

Because the radiation from the plasma is significant enough to be measurable (and indeed forms the basis of the temperature diagnostic fielded on these experiments), it is important to 
verify that it is not large compared to Ohmic heating, as this is one of the primary assumptions in the derivation of striation-form ETI. For typical current densities of $10^{7} \mathrm{~A} / \mathrm{cm}^{2}\left(10^{11} \mathrm{~A} / \mathrm{m}^{2}\right)$ and aluminum resistivity of order $10^{-7} \Omega-\mathrm{m}$ (which is small compared to the resistivity of aluminum vapor), the total power deposited in a $400 \mathrm{~nm} \times 1 \mathrm{~cm} \times 0.9 \mathrm{~cm}$ foil is $\sim 3 \times 10^{4} \mathrm{~W}$. The StefanBoltzmann law gives the total power radiated from a w x L rectangular blackbody surface as

$$
P=\sigma T^{4} w L
$$

where $\sigma=5.67 * 10^{-8} \frac{W}{m^{2} K^{4}}$ is the Stefan-Boltzmann constant. The uniform surface temperature required to equalize blackbody losses to Ohmic heating is $9000 \mathrm{~K}$. Because ETI primarily grows when the bulk material is liquid [19], which for aluminum at atmospheric pressure is the temperature range of 933 to $2743 \mathrm{~K}$, radiative losses can be safely ignored, as these losses are orders of magnitude below Ohmic heating in this temperature range.

\subsubsection{Comparison with $\mathrm{SF}_{6}$ and Vacuum Backgrounds}

Figure 5.7 shows a comparison between self-emission features from a foil ablation in air and $\mathrm{SF}_{6}$ backgrounds at similar times and current densities. The images are similar in brightness and formation time of features, but the features appear qualitatively different. The interpretation for this is that once foil material starts to vaporize, the locally high material pressure propagates in the background gas as a semi-cylindrical shock (the shock is semi cylindrical because the ablating feature is initially a line, and the half space behind the foil is constrained by the solid plastic support structure). The shock velocity in a given medium is $[66,67]$

$$
v_{S}=c_{S}\left(\frac{\gamma-1}{2 \gamma}+\frac{\gamma+1}{2 \gamma} \frac{p_{2}}{p_{1}}\right)^{\frac{1}{2}}
$$

where $c_{s}$ is the speed of sound in the medium, $\gamma$ is the ratio of specific heats, $p_{2}$ is the pressure behind the shock, and $p_{1}$ is the pressure in front of the shock. In $\mathrm{SF}_{6}$, the sound speed is $133 \mathrm{~m} / \mathrm{s}$ 
and $\gamma$ is approximately 1.1 , and in air, the sound speed is $343 \mathrm{~m} / \mathrm{s}$ and $\gamma$ is 1.4 . For a very large range of shock pressures ( $p_{2}=10$ to 10,000 atmospheres), the ratio of shock speed in air to the ratio of shock speed in $\mathrm{SF}_{6}$ is a constant value of 2.44. This difference in propagation speed of plasma features likely explains the qualitative difference in feature shape observed in Figure 5.7; the shock moves slower in the heavier $\mathrm{SF}_{6}$, keeping the vaporized material in electrical contact longer and allowing more electrical energy to be deposited into these regions before they disperse. (This effect of heavier background gas confining ablated material was found by Ventzek [68] and Ching [69] in laser ablation experiments.) The formation time of striations is identical because the underlying physics of vaporization and ionization of the aluminum is the same regardless of gas background. 


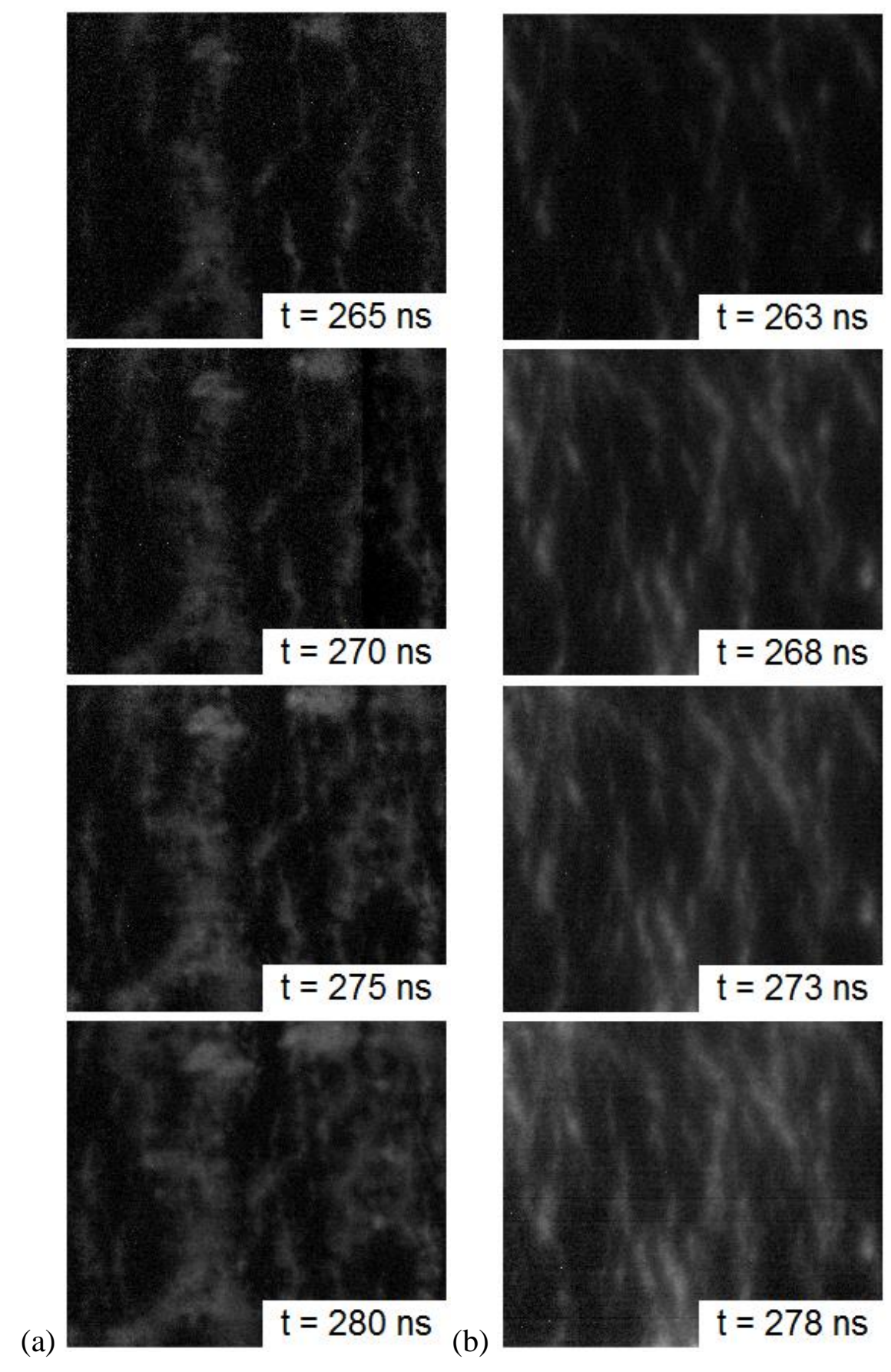

Figure 5.7: (a) Partial image sequence showing self-emission from a $400 \mathrm{~nm} \times 1.0 \mathrm{~cm} \times 0.9 \mathrm{~cm}$ foil ablating in air (b) Partial image sequence showing a foil of the same dimensions ablating in atmospheric pressure $\mathrm{SF}_{6}$. All frames are $1.25 \mathrm{~mm} \times 1.08 \mathrm{~mm}$ with $5 \mathrm{~ns}$ exposure and $2 \mu \mathrm{m}$ spatial resolution. Current flows from left to right.

Results from an electrical ablation performed in vacuum at $3 \times 10^{-5}$ Torr are shown in Figure 5.8. Total light emission levels in vacuum were considerably lower, requiring $150 \mathrm{~ns}$ 
exposures to be employed to observe the formation of striations. Unlike typical atmospheric ablations, such as the one shown in Figure 5.6, the vacuum ablation exhibited only transient striation formation with no merging or formation of parallel-to-current plasma channels. There are two reasons for these lower intensity, short-lived striations. First, the vaporization temperature of aluminum at $3 \times 10^{-5}$ Torr is less than $1100 \mathrm{~K}$ [70], considerably lower than the atmospheric pressure value of $2600 \mathrm{~K}$. This causes the transition to vapor to occur at a temperature below the threshold for what can be viewed on the framing camera diagnostic for reasonable exposure times. Second, the vacuum dramatically increases the expansion velocity of the exploding aluminum vapor compared to shock expansion in ambient air [68]. This has the opposite effect of the argon background discussed in Figure 5.7; sections of the foil that vaporize quickly blow apart, resulting in a shorter window over which electrical energy can be input into the vaporized material. The result of these processes is short-lived, dim bands of plasma that do not have time to form into full plasma channels before the foil has blown apart. This analysis was also supported by visually inspecting the vacuum window after the shot; large solid pieces of aluminum foil were implanted on the window, indicating the foil had disassembled before the bulk material had vaporized. These large residual pieces were never observed on the plastic shield (located at a similar position relative to the load as the vacuum window) for atmospheric pressure ablations. 

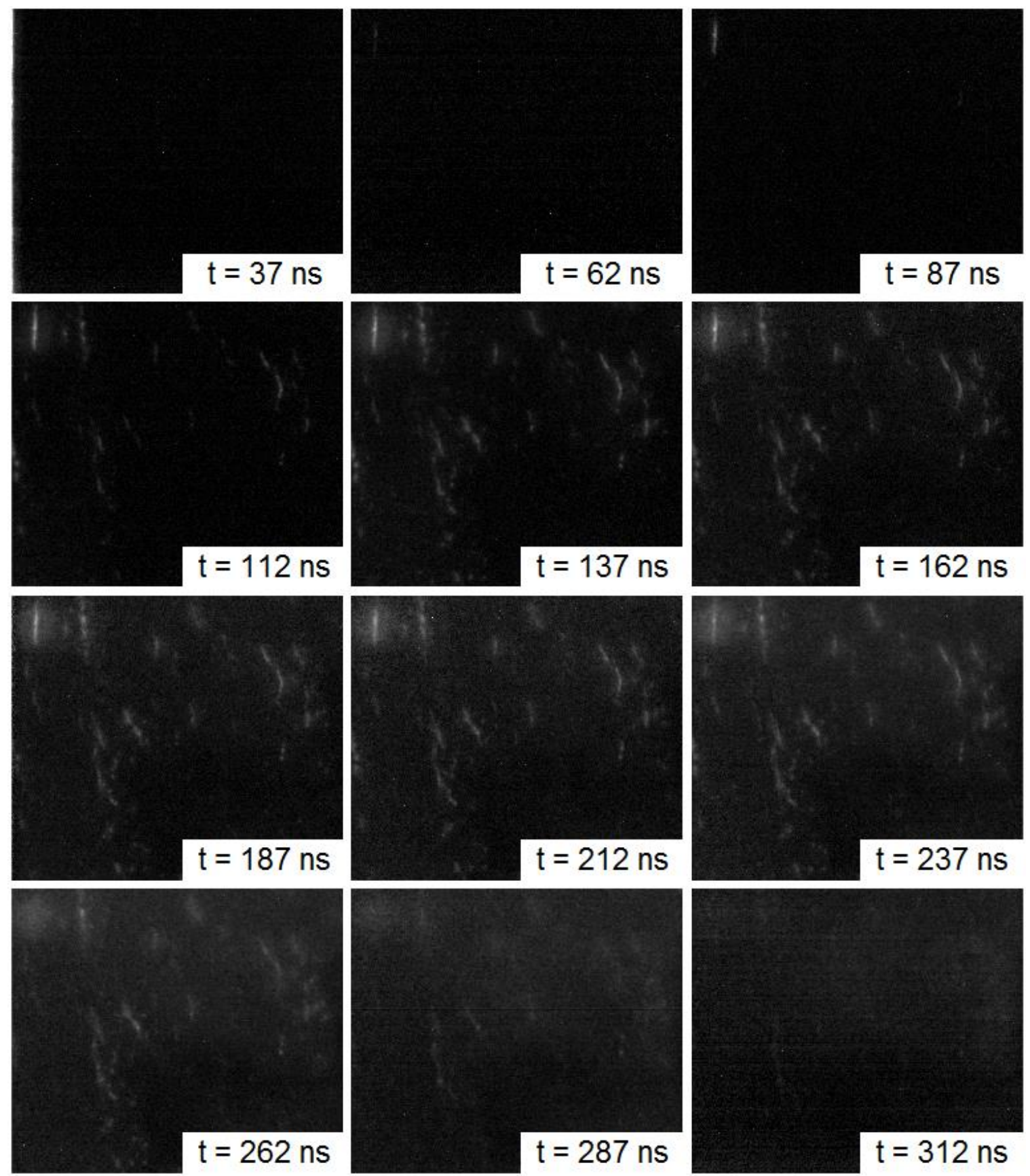

Figure 5.8: Self-emission sequence of a $400 \mathrm{~nm} \times 1.0 \mathrm{~cm} \times 0.9 \mathrm{~cm}$ foil ablation in vacuum. Exposure time for each image is $150 \mathrm{~ns}$, and the imaging window is the maximum available $5 \mathrm{~mm} \times 4.32 \mathrm{~mm}$ ( $10 \mu \mathrm{m}$ resolution) to maximize light collection. Current flows from left to right. 


\section{3: Temperature Measurements of Ablating Foils}

Framing camera imaging sequences of ablating foils were converted into temperature data using the response calibrations shown in Figure 3.13. As described in Section 3.3, the exposure time and pixel size of each image establishes a resolvable temperature range, above which the camera reads saturation and below which the camera reads zero. Resistivity and phase plots, such as those shown in Figure 5.5, indicate that the formation of bright filamentary structures consistently occurs before the bulk foil has vaporized. It is therefore anticipated that the "dark" regions of framing camera images are at a temperature corresponding to the vaporization temperature of aluminum at atmospheric pressure, which is $2743 \mathrm{~K}$. To verify this, several shots were performed with long (>40 ns) exposure times in order to resolve these relatively low temperatures at the cost of temporal resolution. Figure 5.9 shows a temperature plot from one of these long-exposure images.

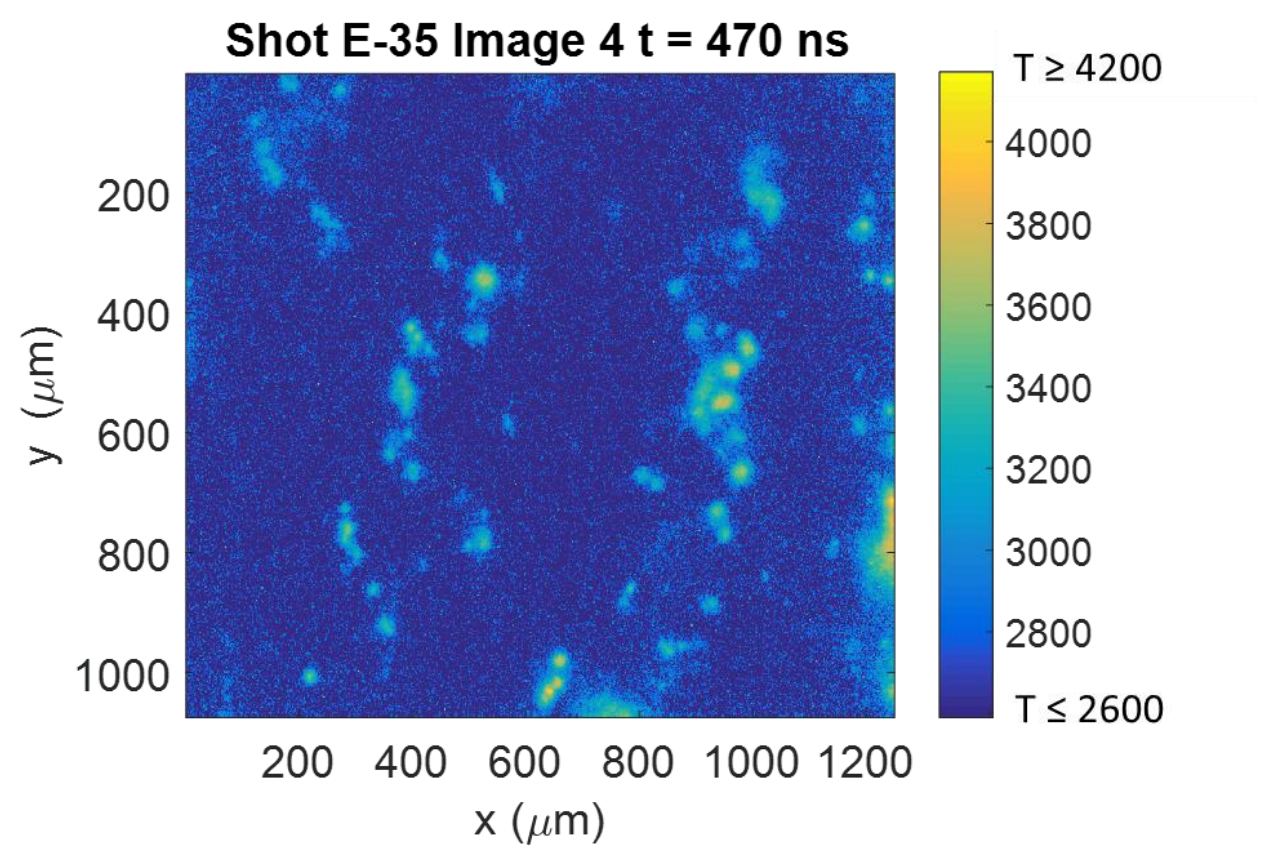

Figure 5.9: Temperature plot (in Kelvin) of an $80 \mathrm{~ns}$ exposure image, taken on an $800 \mathrm{~nm} \times 1.0 \mathrm{~cm} \times 0.9 \mathrm{~cm}$ aluminum foil to establish background temperature during formation of striation structures. For this exposure and magnification, the camera response floor corresponds to $2600 \mathrm{~K}$, and saturation corresponds to $4200 \mathrm{~K}$. Current travels left to right. 
Figure 5.9 shows striations beginning to form, with the hottest spots exceeding the saturation temperature of $4200 \mathrm{~K}$. Far from these striations, the temperature of the material is in the $2600 \mathrm{~K}$ to $2800 \mathrm{~K}$ range, showing excellent agreement with the predicted $2743 \mathrm{~K}$ background. This measurement also implies the estimated $500 \mathrm{~K}$ error of the temperature measurement due to uncertainty in the emissivity of biphase liquid/vapor aluminum is quite conservative.

Figures 5.10 through 5.14 show temperature surface plot sequences for $1.25 \mathrm{~mm} \times 1.08$ $\mathrm{mm}$ sections of various foils. Image times were chosen to attempt to capture all three phases (striation formation, merging, and plasma channel formation) of the ablation on each sequence. The shots represented in these figures were chosen to represent typical features observed on the five foil geometries most commonly used in experiments; these dimensions are $400 \mathrm{~nm}$ x $1.0 \mathrm{~cm}$, $800 \mathrm{~nm} \times 0.7 \mathrm{~cm}, 800 \mathrm{~nm} \times 1.0 \mathrm{~cm}, 2.0 \mu \mathrm{m} \times 0.25 \mathrm{~cm}$, and $2.0 \mu \mathrm{m} \times 0.4 \mathrm{~cm}$. As the electrodes holding the foils in place are spaced $0.9 \mathrm{~cm}$ apart, the ablating section of foil is $0.9 \mathrm{~cm}$ long on every shot. 


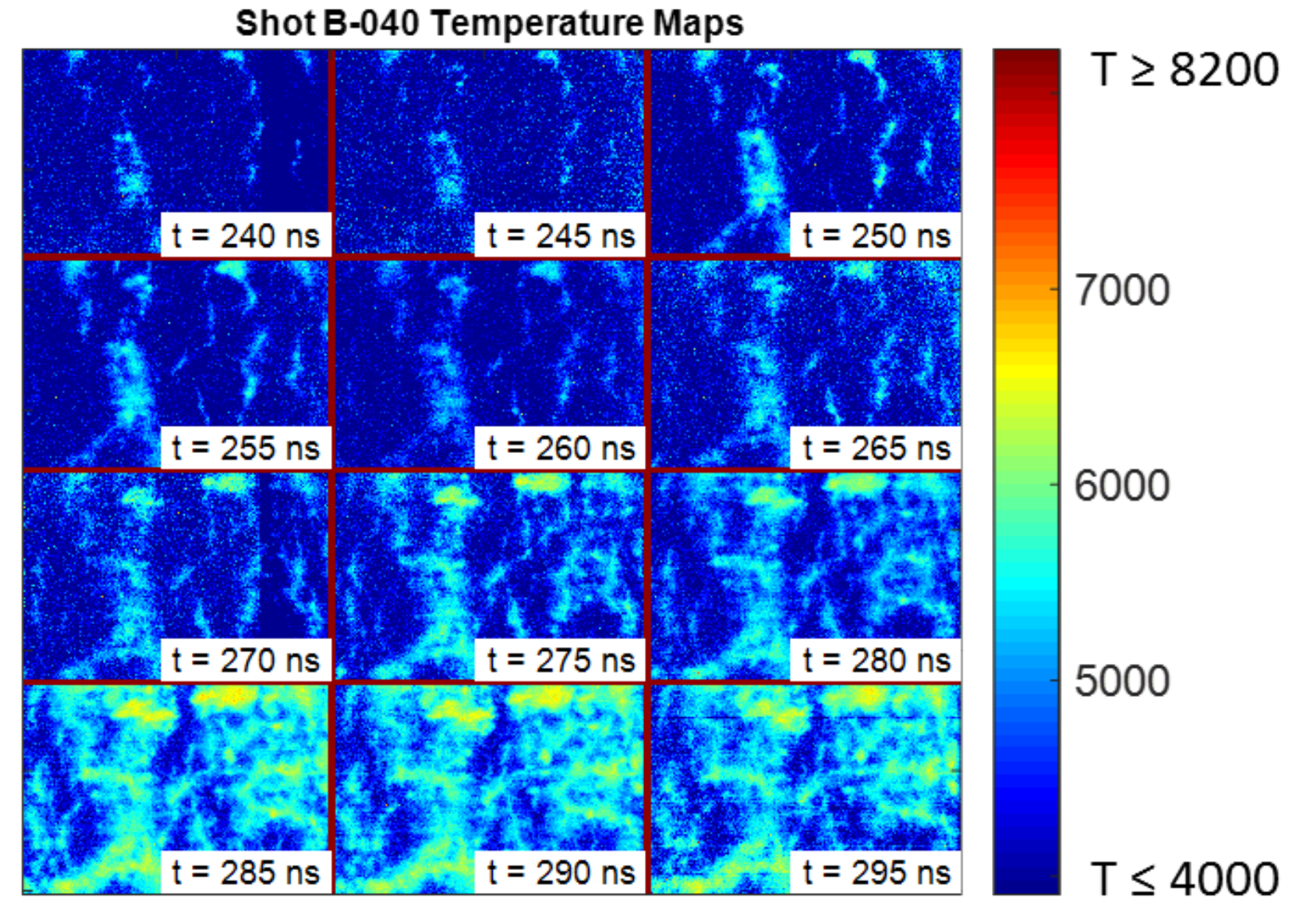

Figure 5.10: Temperature surface maps (in $K$ ) for a $400 \mathrm{~nm}$ x $1.0 \mathrm{~cm}$ x $0.9 \mathrm{~cm}$ foil; $\mathrm{J} \approx 5.0 \times 10^{7} \mathrm{~A} / \mathrm{cm}^{2}$. Image sizes are $1.25 \mathrm{~mm}$ by $1.08 \mathrm{~mm}$. Current flows from left to right. 
Shot E-14 Temperature Maps

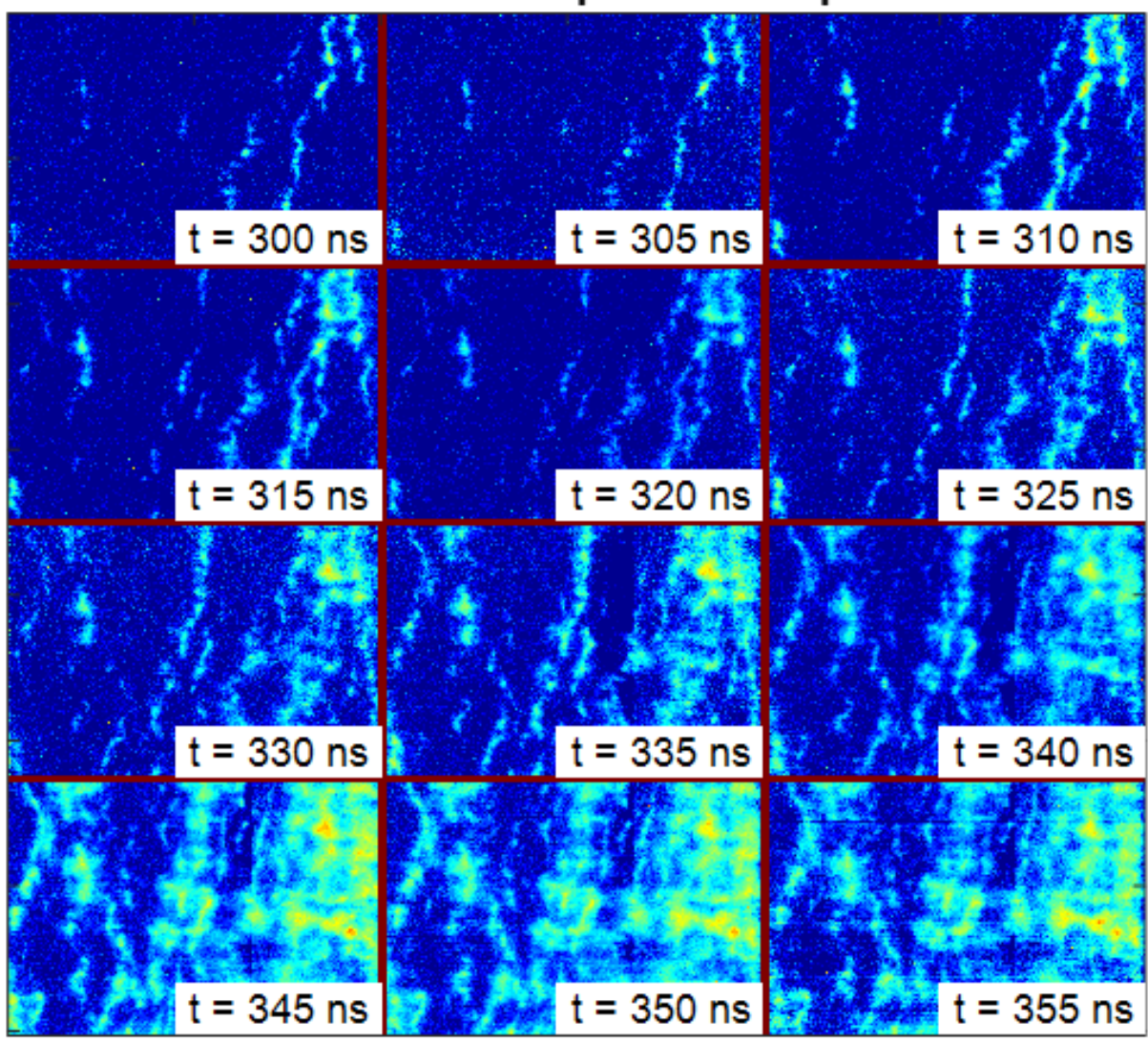

\section{$T \geq 8200$}

7000

6000

5000

\section{$\mathrm{T} \leq 4000$}

Figure 5.11: Temperature surface maps (in $\mathrm{K}$ ) for an $800 \mathrm{~nm}$ x $0.7 \mathrm{~cm}$ x $0.9 \mathrm{~cm}$ foil; $\mathrm{J} \approx 4.0 \times 10^{7} \mathrm{~A} / \mathrm{cm}^{2}$. Image sizes are $1.25 \mathrm{~mm}$ by $1.08 \mathrm{~mm}$. Current flows from left to right. 


\section{Shot E-024 Temperature Maps}

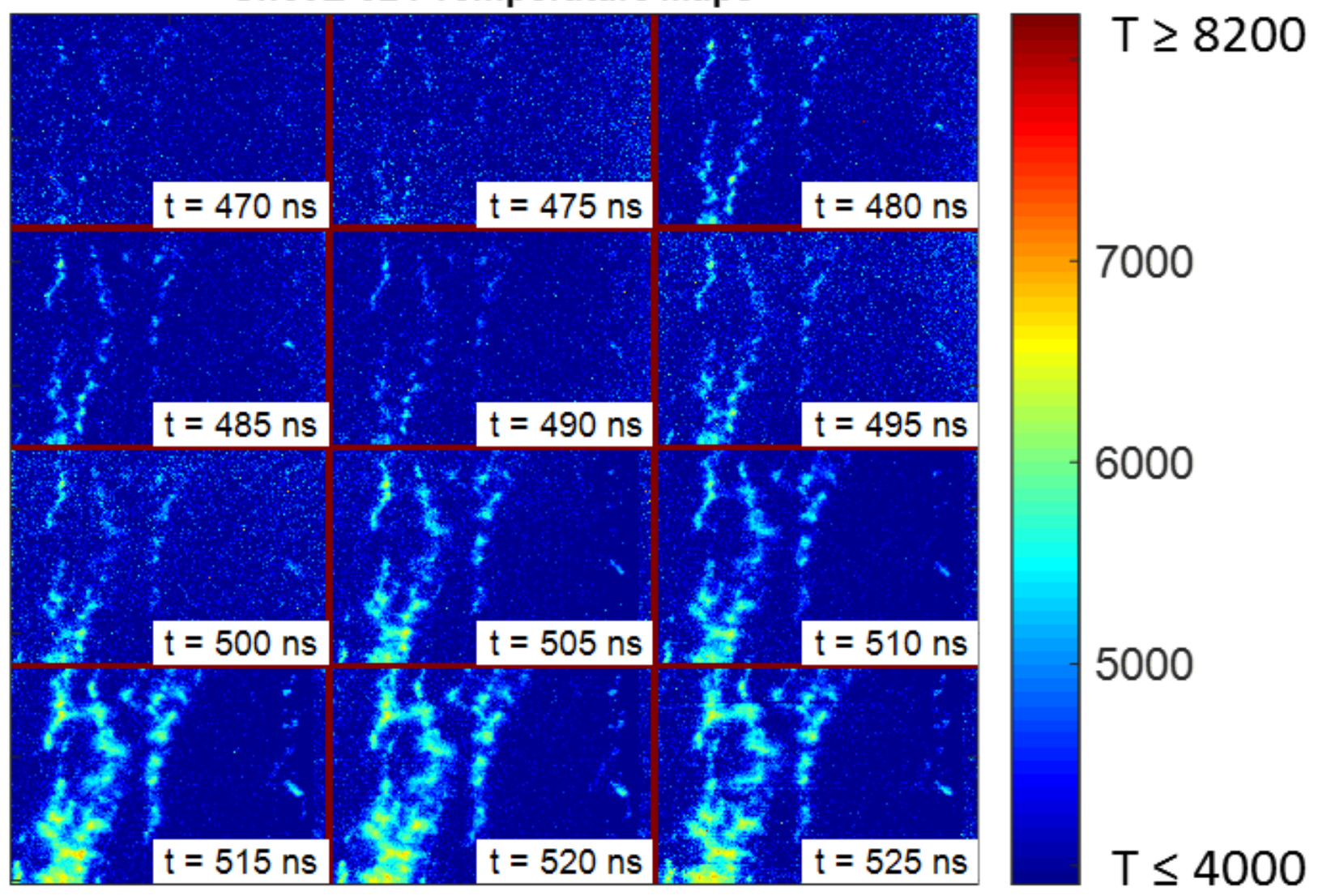

Figure 5.12: Temperature surface maps (in $\mathrm{K}$ ) for an $800 \mathrm{~nm}$ x $1.0 \mathrm{~cm}$ x $0.9 \mathrm{~cm}$ foil; $\mathrm{J} \approx 3.9 \times 10^{7} \mathrm{~A} / \mathrm{cm}^{2}$. Image sizes are $1.25 \mathrm{~mm}$ by $1.08 \mathrm{~mm}$. Current flows from left to right. 


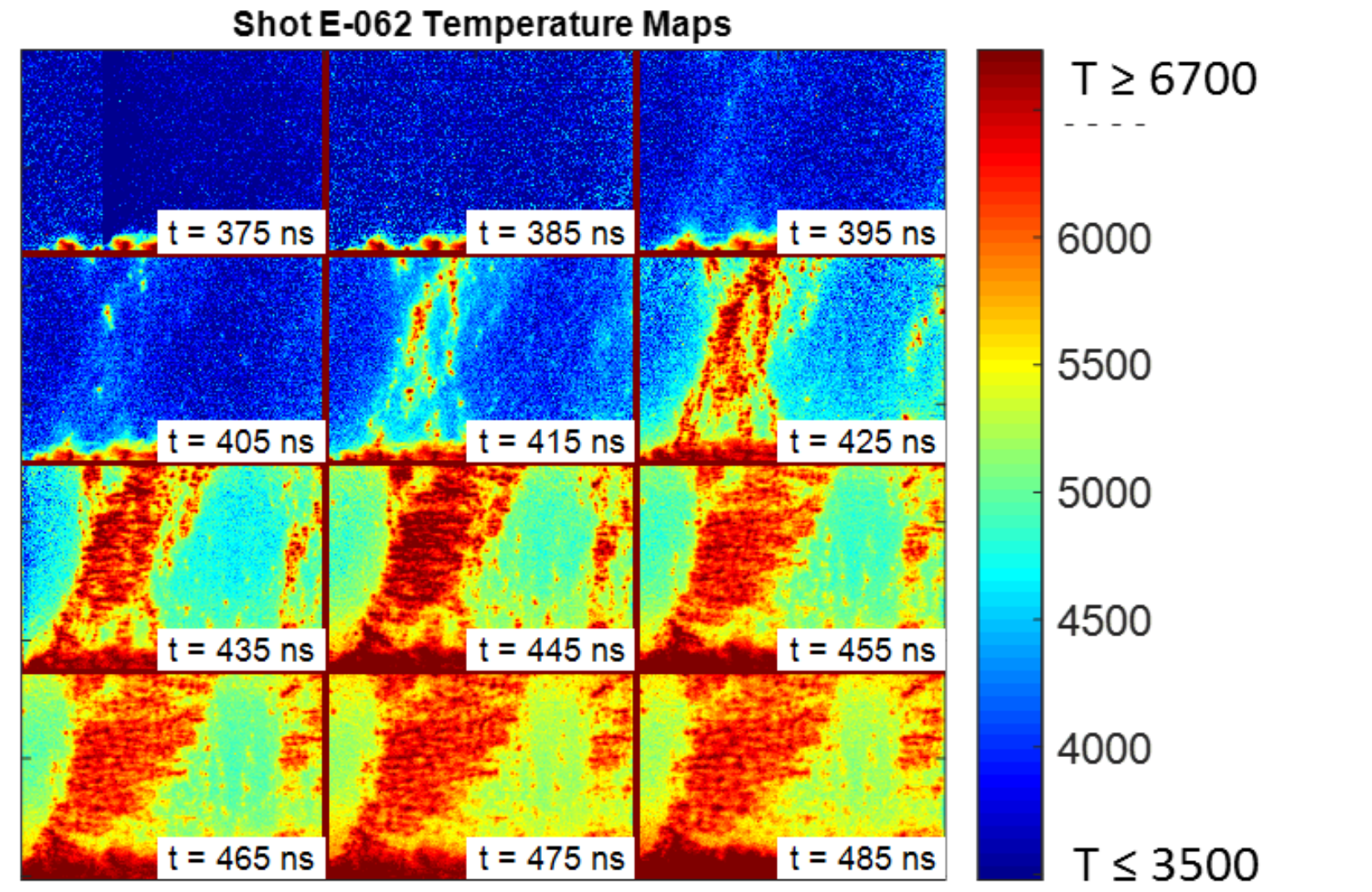

Figure 5.13: Temperature surface maps (in $\mathrm{K}$ ) for a $2.0 \mu \mathrm{m} \times 0.25 \mathrm{~cm}$ x $0.9 \mathrm{~cm}$ foil; $\mathrm{J} \approx 6.0 \times 10^{7} \mathrm{~A} / \mathrm{cm}^{2}$. Image sizes are $1.25 \mathrm{~mm}$ by $1.08 \mathrm{~mm}$. Current flows from left to right. 


\section{Shot E-027 Temperature Maps}

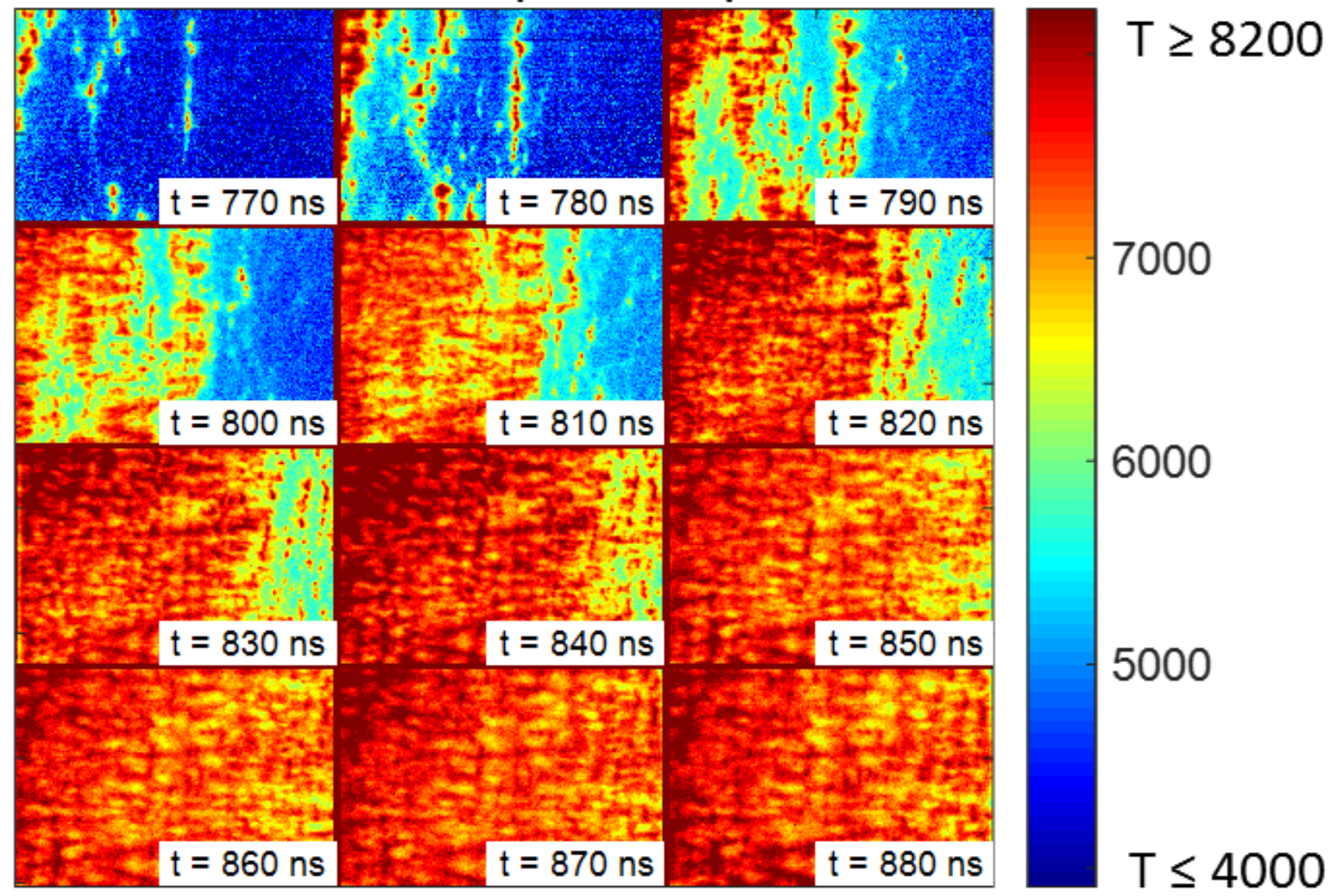

Figure 5.14: Temperature surface maps (in $\mathrm{K}$ ) for a $2.0 \mu \mathrm{m} \times 0.4 \mathrm{~cm} \times 0.9 \mathrm{~cm}$ foil; $\mathrm{J} \approx 3.5 \times 10^{7} \mathrm{~A} / \mathrm{cm}^{2}$. Image sizes are $1.25 \mathrm{~mm}$ by $1.08 \mathrm{~mm}$. Current flows from left to right.

Figures 5.10 through 5.12 show qualitatively similar features. Filaments on the $400 \mathrm{~nm}$ and $800 \mathrm{~nm}$ foils had similar initial widths, which were consistently on order 10's of microns; the smallest observed growing structures were approximately $15 \mu \mathrm{m}$, corresponding to a peak-topeak wavelength of $30 \mu \mathrm{m}$. Current densities at the time of filament formation were similar, a likely consequence of the conservation of action integral $\int J^{2} d t[19,71]$ for explosions occurring during the risetime of the current pulse. This $30 \mu \mathrm{m}$ observed minimum observed growing wavelength is a factor of two or three greater than the theoretical $\lambda_{\min }$ shown on Figure 2.12 for the times imaged, agreeing well with previous simulations [19], which anticipate growth on the order of $\gamma_{m}$ for $\lambda \approx 3 \lambda_{\min }$. 
While a similar minimum, growing wavelength was observed for shots using the $2.0 \mu \mathrm{m}$ foils, striations on these foils grew in spatial extent much faster than those on the $400 \mathrm{~nm}$ and $800 \mathrm{~nm}$ foils, forming the large wavelength structures most clearly visible on Figure 5.13. The expanding plasma from these foils often filled up an entire 10x imaging frame $(1.25 \mathrm{~mm} \times 1.08$ $\mathrm{mm}$ ) late in time. Current densities are not dramatically different on the $2.0 \mu \mathrm{m}$ foils, and the foils in Figure 5.12 and Figure 5.14 have the same mass, so the qualitative differences in ablation dynamics are likely due to the foils themselves. Figure 5.12 indicates large wavelength ( $\gtrsim 100$ $\mu \mathrm{m})$ perturbations can grow starting at $\mathrm{t}=0$, so one possible explanation for the wide striations on the $2.0 \mu \mathrm{m}$ foils is a thickness perturbation from the rolling process that fabricates the foils. While a thickness perturbation would not perturb resistivity as much as poorly conducting impurity inclusions such as silicon, the largest impurity inclusions are on $\sim 10 \mu \mathrm{m}$ spatial scales, observable on preshot images such as Figure 3.8. Larger wavelength perturbations in thickness intrinsic to the $2.0 \mu \mathrm{m}$ foils could grow temperature perturbations over the entire current rise, allowing these perturbations to reach amplitude on the order of those reached by striations due to resistivity perturbations during the foil vaporization.

\subsection{Analysis of Instability Spatial and Temporal Growth}

\subsubsection{Wavelength Fourier Analysis}

The following procedure was employed to obtain wavelength spectra of temperature perturbations on the foil ablation images. For each of the 12 temperature map images in a given shot sequence, a 10x1-pixel binning algorithm was applied in the perpendicular-to-current direction (vertical on the images) to locally average the signal. This process reduces the impact of individual saturated pixels that appeared in small numbers on all camera images due to noise while still preserving resolution in the parallel-to-current (horizontal) direction. Each original 
860x 1000-pixel image is therefore split into 86 horizontal lineouts. A fast Fourier transform

(FFT) is applied to each lineout to extract wavelength components. Example lineouts are shown in Figure 5.15 for an $800 \mathrm{~nm} \times 0.7 \mathrm{~cm} \times 0.9 \mathrm{~cm}$ foil at four times over the course of the ablation.
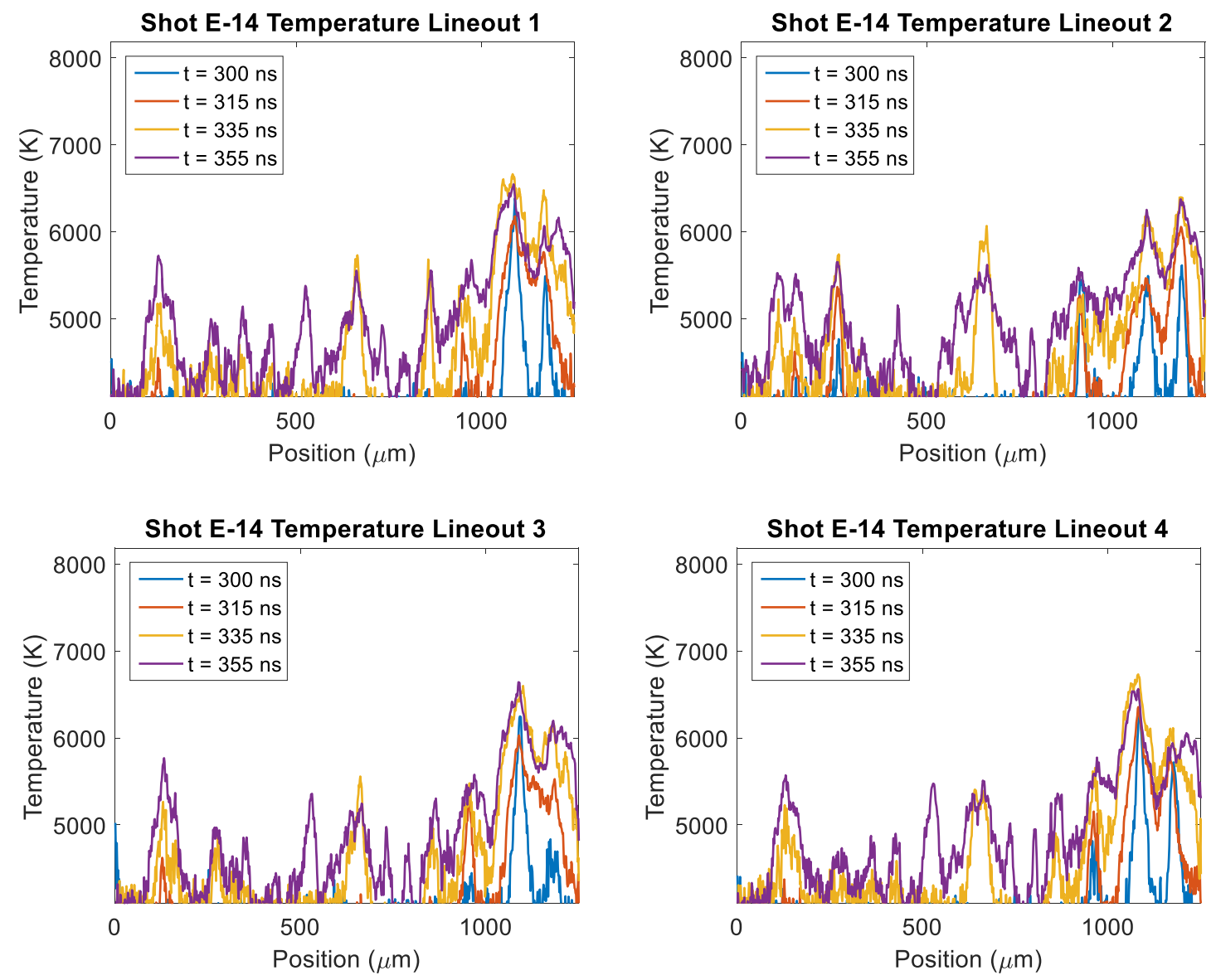

Figure 5.15: Example temperature lineouts of shot E-14 (shown in Figure 5.11) plotted for multiple times during the image sequence. The y-axes are truncated at $4000 \mathrm{~K}$ because temperatures below this value cannot be resolved for the specified magnification and exposure time.

Figures 5.16 through 5.20 show Fourier transforms from the 4 highest-temperature lineouts from the image sequences shown in Figures 5.10 through 5.14. 

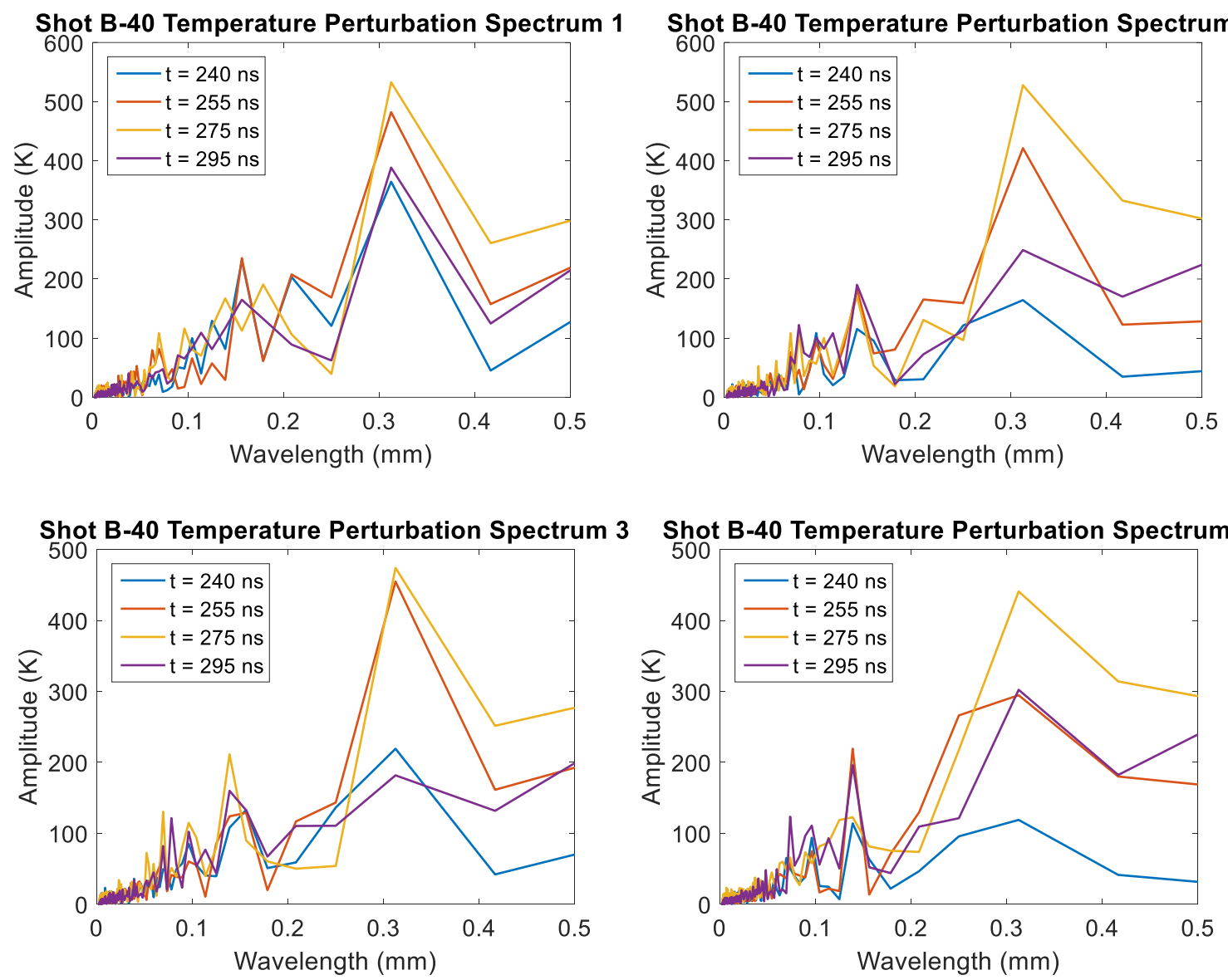

Figure 5.16: Fourier transforms of temperature lineouts of shot B-40 $(400 \mathrm{~nm} \times 1.0 \mathrm{~cm} \times 0.9 \mathrm{~cm}$ foil shown in Figure 5.10), plotted for multiple times during the image sequence. 

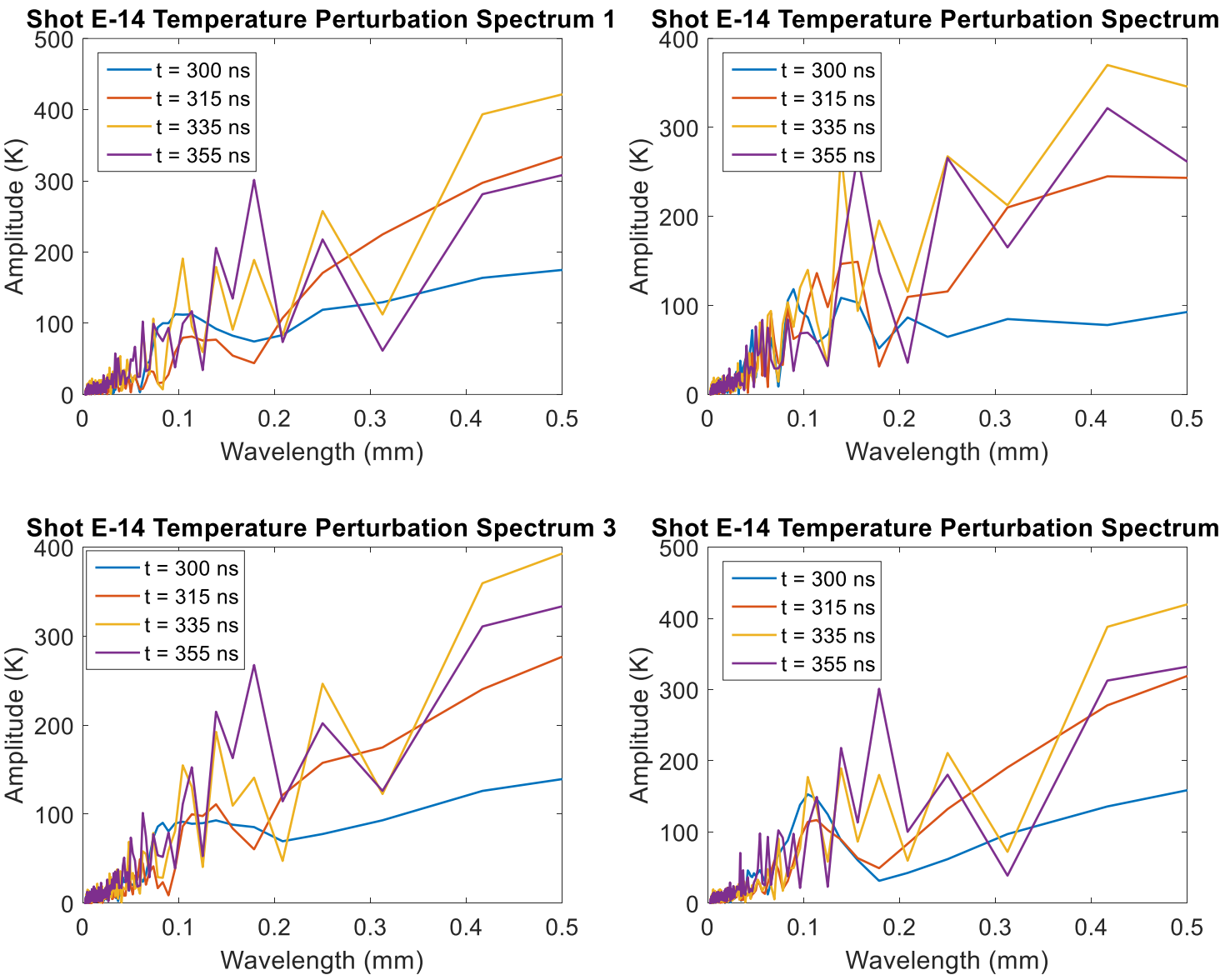

Figure 5.17: Fourier transforms of temperature lineouts of shot E-14 $(800 \mathrm{~nm}$ x $0.7 \mathrm{~cm}$ x $0.9 \mathrm{~cm}$ foil shown in Figure 5.11), plotted for multiple times during the image sequence. 

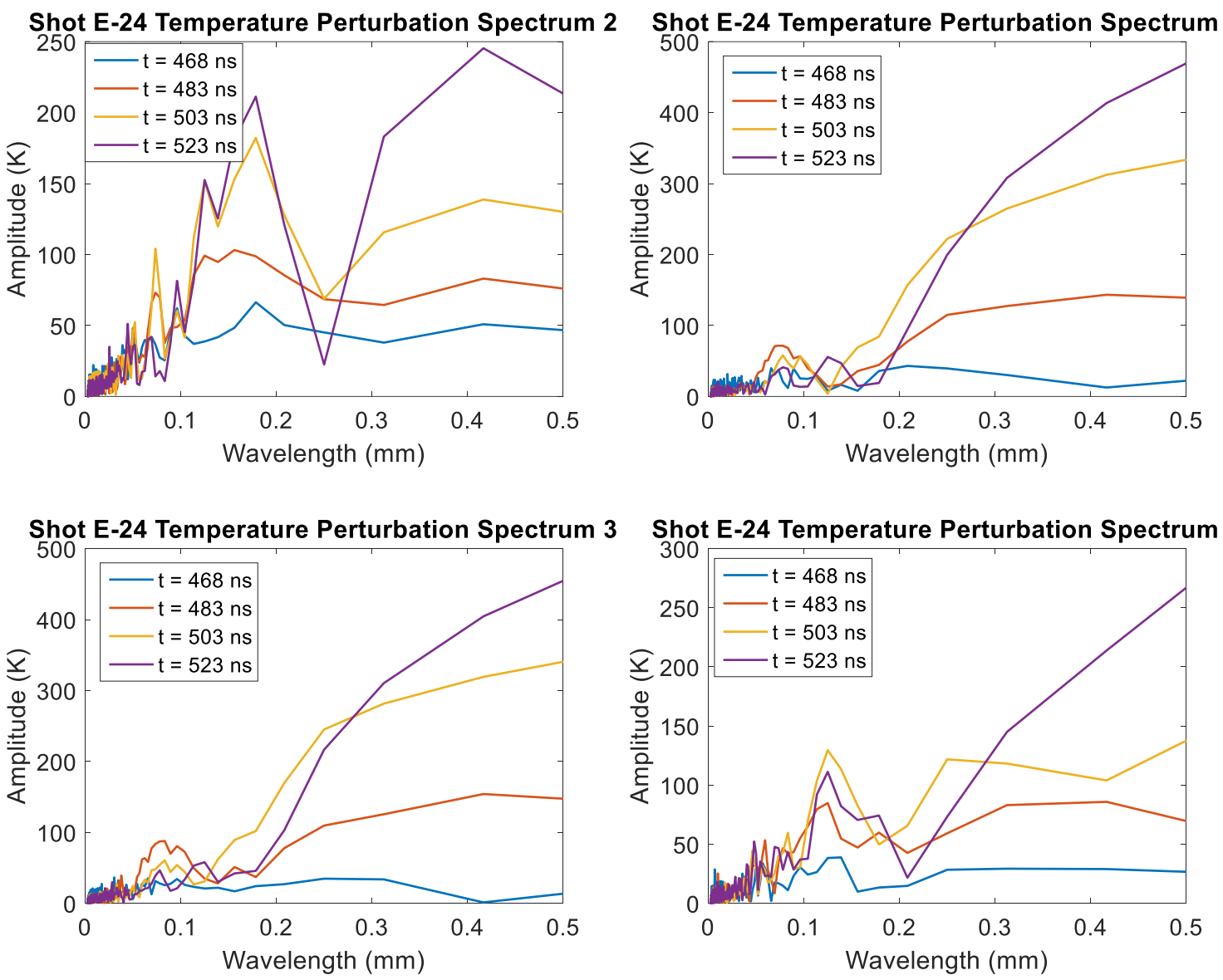

Figure 5.18: Fourier transforms of temperature lineouts of shot E-24 $(800 \mathrm{~nm}$ x $1.0 \mathrm{~cm}$ x $0.9 \mathrm{~cm}$ foil shown in Figure 5.12), plotted for multiple times during the image sequence. 

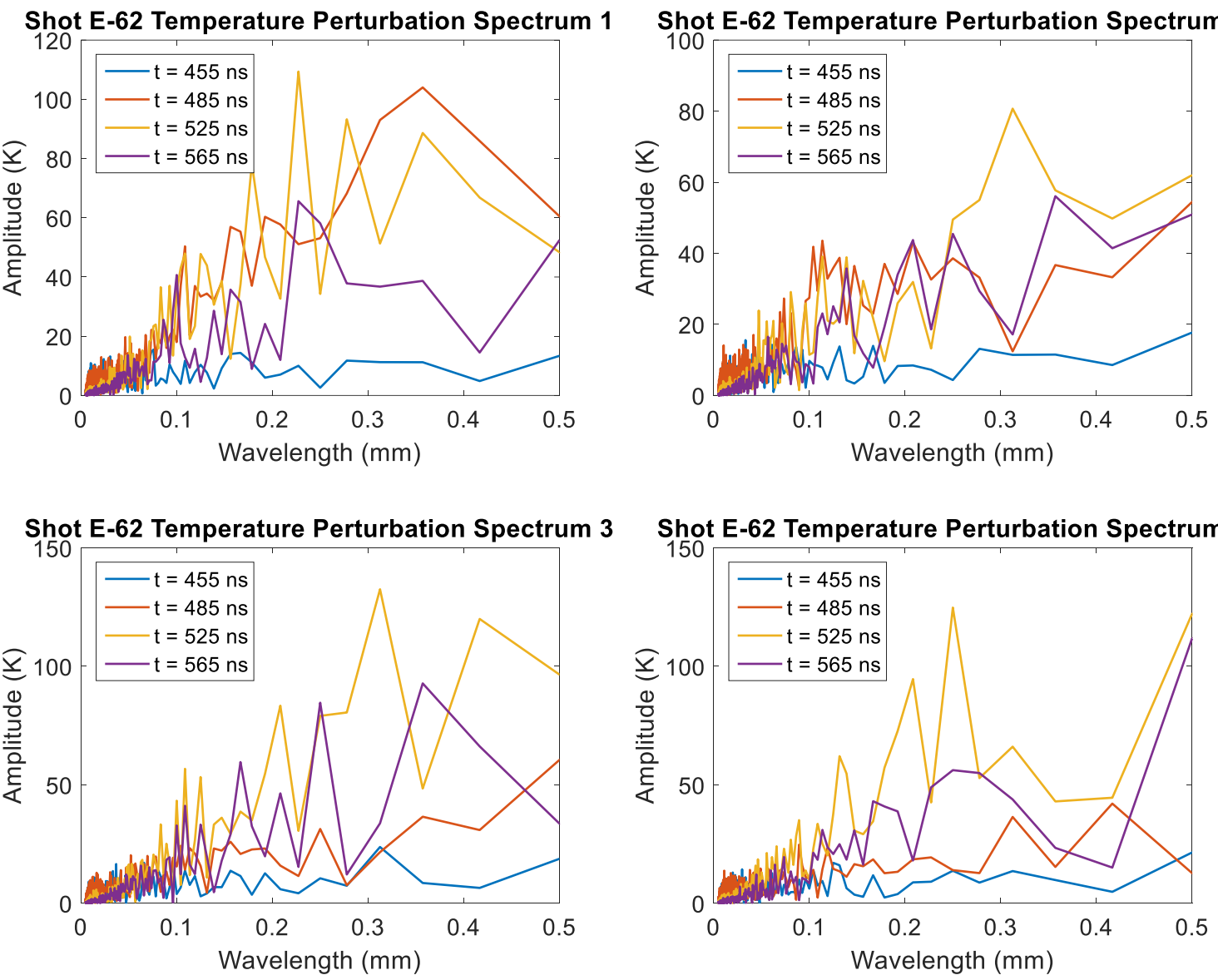

Figure 5.19: Fourier transforms of temperature lineouts of shot E- $62(2.0 \mu \mathrm{m} \times 0.25 \mathrm{~cm} \times 0.9 \mathrm{~cm}$ foil shown in Figure 5.13), plotted for multiple times during the image sequence. 

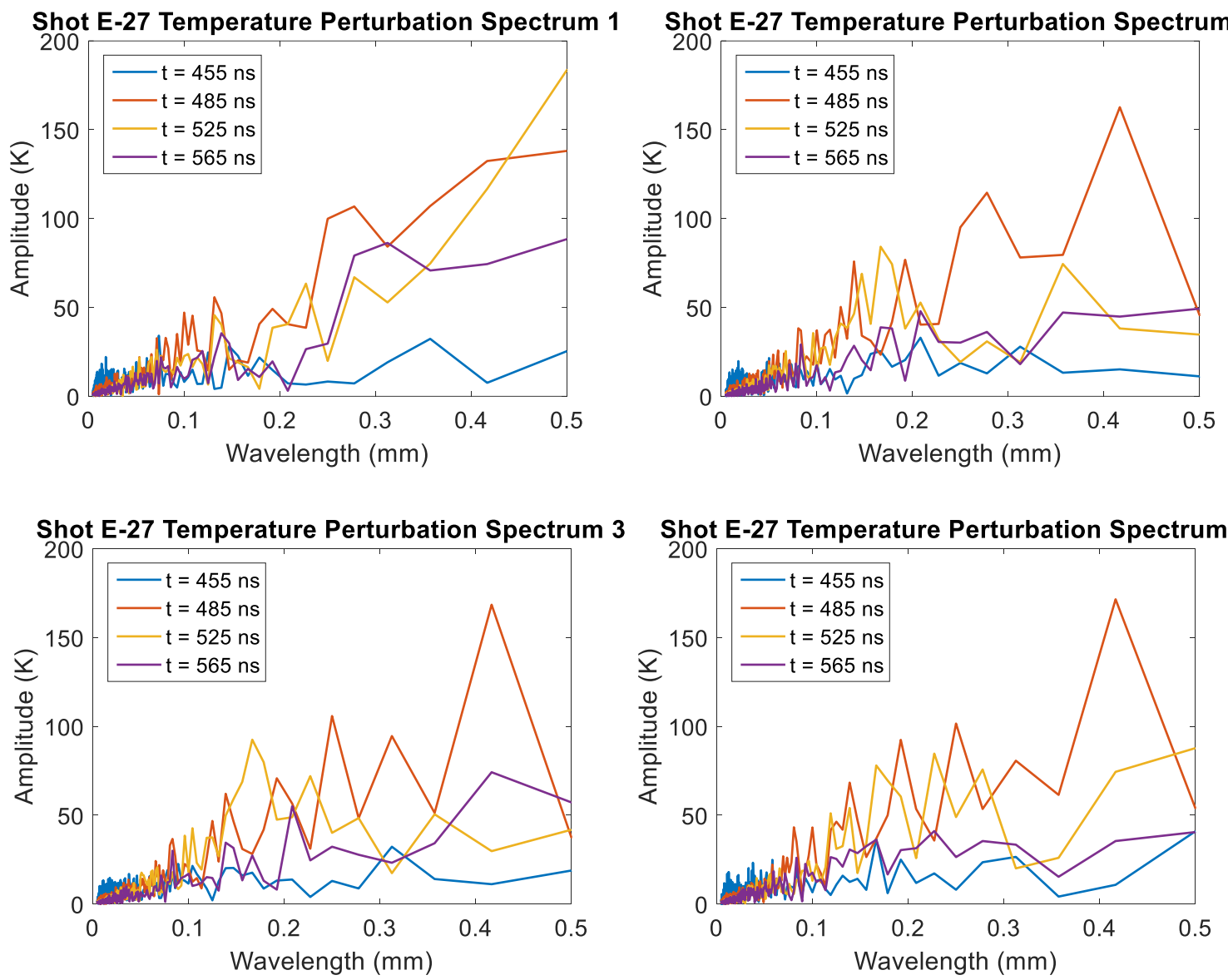

Figure 5.20: Fourier transforms of temperature lineouts of shot E- $62(2.0 \mu \mathrm{m} \times 0.25 \mathrm{~cm} \times 0.9 \mathrm{~cm}$ foil shown in Figure 5.13), plotted for multiple times during the image sequence.

Figures 5.16 through 5.20 show quantitatively the trends observed in Figures 5.10 through 5.14. On the shots with the $400 \mathrm{~nm}$ and $800 \mathrm{~nm}$ foils, early time images have perturbations largely in the 10 's of $\mu \mathrm{m}$ range, while later images transition to dominant wavelengths between 200 and $500 \mu \mathrm{m}$. The $2.0 \mu \mathrm{m}$ foils have significantly less contribution from sub-100 $\mu \mathrm{m}$ wavelengths, and late in time the amplitude of the Fourier transforms drops as the majority of the window saturates the camera response. 


\subsubsection{Growth Rate Measurements}

Thirteen shots were identified with image timing taking place during the growing temperature perturbation phase on all frames (i.e. no all-black or all-saturated frames in the sequence) and selected for growth rate analysis. From the analysis described in Section 5.1, it was determined that the observation window on all 13 of these shots occurred entirely before the bulk foil had sufficient enthalpy to complete vaporization; therefore, the background temperature (the temperature of the "dark" regions) was assumed to be the vaporization temperature of aluminum, $2743 \mathrm{~K}$. To measure growth rate, the highest amplitude point was found on each lineout from an intermediate image on the sequence, and the temperature of this point was monitored as a function of time. Growth rate was calculated for each point by fitting timedependent temperature to an exponential curve. An average of $\sim 20$ of these growth rates per shot was taken to represent the average growth rate for the observation window of that shot. Points that included values below the measurement floor or above saturation were rejected because the inclusion of these points artificially lowered the measured growth rate. Figure 5.21 shows a sample of growth rate calculations performed for the four lineouts presented in Figure 5.15. 

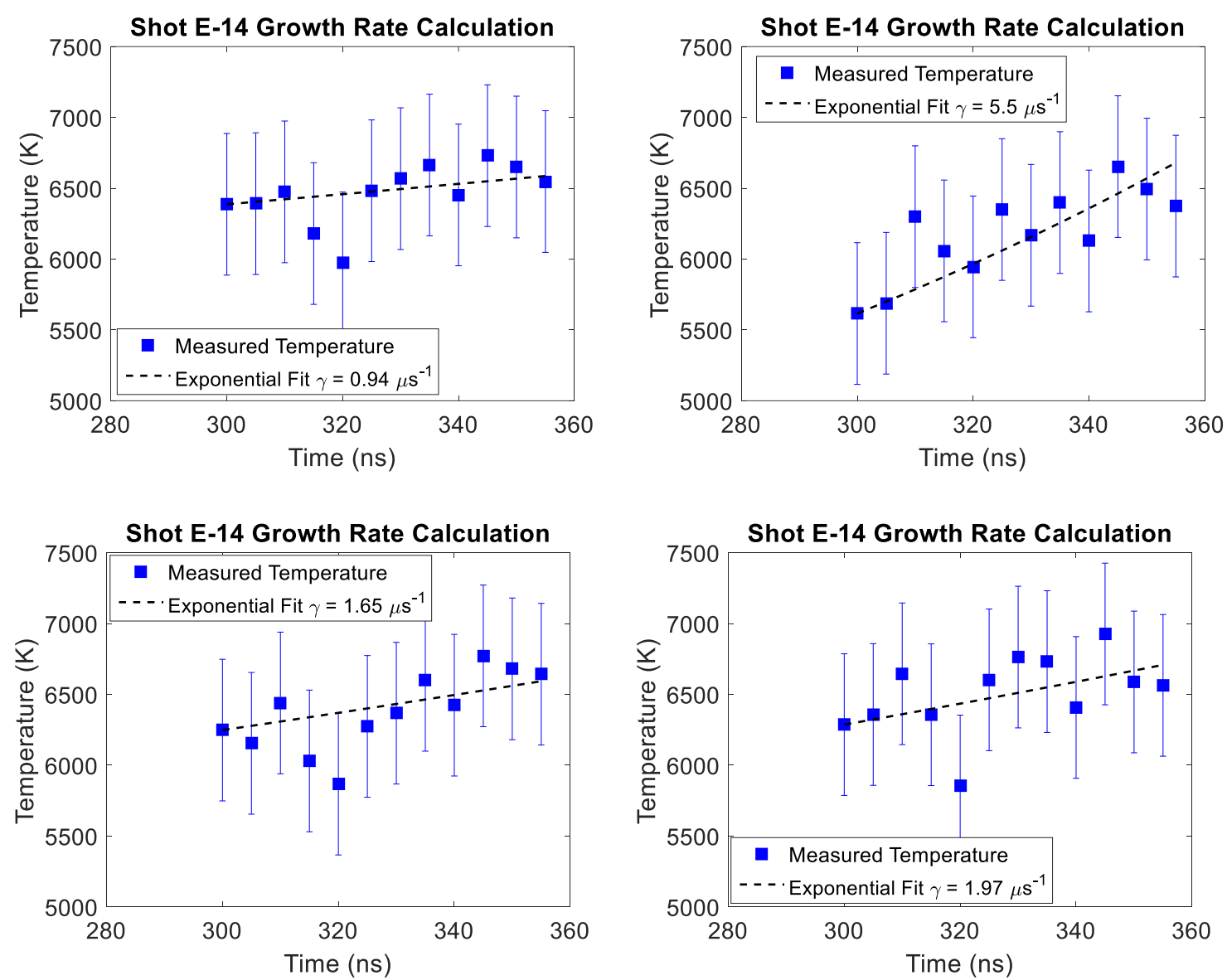

Fig 5.21: Growth rate calculations for lineouts shown in Figure 5.15. Amplitude is plotted as a function of time and fit to an exponential function; the coefficients of several of these functions are then averaged to obtain an average growth rate for the shot.

The measured average growth rate is then compared to theoretical ETI growth rates calculated as in Section 2.4 using the measured current and material properties interpolated or extrapolated from (2.38). Figure 5.22 shows sample results from these comparisons. On Figure 5.22, the green box represents the observation window of the 12 -frame ICCD, the dashed black line indicates the measured averaged growth rate during this window, and the colored curves indicate calculated growth rates for various wavelengths greater than $\lambda_{\min }$. 

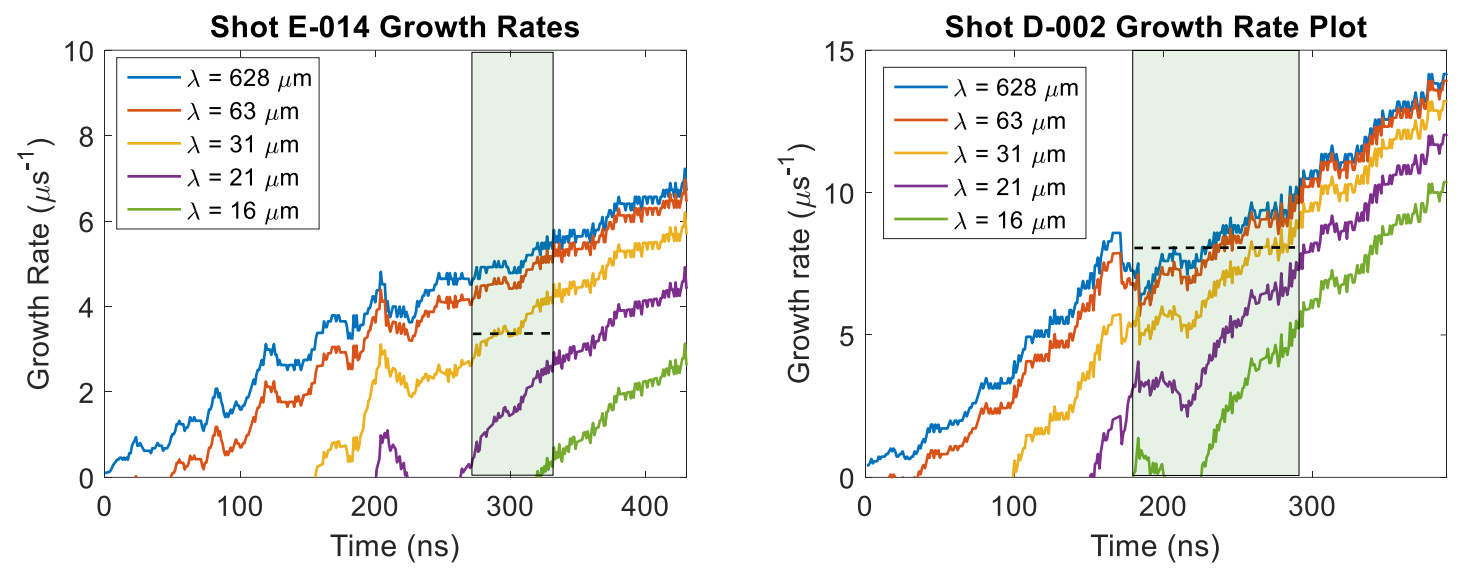

Fig 5.22: Instantaneous growth rates calculated from (2.18) with the imaging window indicated as a green box. The measured average growth rate is shown as a dashed horizontal line to compare with the theoretical values. The blue curve, which corresponds to $k=10^{4} \mathrm{~m}^{-1}$, is taken to represent $\gamma_{\max }$, as the corresponding wavelength is nearly two orders of magnitude greater than $\lambda_{\min }$. The average growth rate in the observation window is expected to fall between the average value within this window for $\lambda=30 \mu \mathrm{m}$ (represented by the yellow curve) and the average value of $\gamma_{\max }$ (represented by the blue curve).

Figure 5.22 shows the measured average growth rates agree with calculated ETI growth rates in the range of $\lambda=30 \mu \mathrm{m}$, the smallest experimentally observed growing wavelength, to $\lambda=628 \mu \mathrm{m}$, an effectively infinite wavelength corresponding to maximum growth with no reduction due to thermal conductivity. Generally, measured average growth rates were found to fall within, or at least close to, the range bounded by the calculated growth rates $\gamma_{30}$ (the growth rate for $\lambda=30 \mu \mathrm{m}$ ) and $\gamma_{\max }$. Table 5.1 shows the growth rate results from all 13 shots along with the mass and thickness of each foil and the average current density during the observation window. 
Table 5.1: Measured ETI growth rates for various foil masses and geometries, listed along with theoretical growth rates for long-wavelength modes (the maximum growth rate predicted by theory) and theoretical growth rates for $\lambda=$ $30 \mu \mathrm{m}$ (a consistently observed smallest growing feature size that appeared to be independent of foil mass).

\begin{tabular}{|c|c|c|c|c|c|c|}
\hline $\begin{array}{c}\text { Shot } \\
\text { Number }\end{array}$ & $\begin{array}{c}\text { Foil mass } \\
(\mu g)\end{array}$ & $\begin{array}{c}\text { Thickness } \\
(\mathrm{nm})\end{array}$ & $\begin{array}{c}\mathrm{J} / 1 \mathrm{e} 7 \\
\left(\mathrm{~A} / \mathrm{cm}^{2}\right)\end{array}$ & $\begin{array}{c}\text { Measured Growth } \\
\text { Rate }\left(\mu \mathrm{s}^{-1}\right) \\
\end{array}$ & $\begin{array}{c}\gamma_{\max } \\
\left(\mu \mathbf{s}^{-1}\right)\end{array}$ & $\begin{array}{c}\gamma_{30} \\
\left(\mu \mathrm{s}^{-1}\right)\end{array}$ \\
\hline E23 & 194 & 800 & 3.7 & 4.9 & 4.3 & 3 \\
\hline E29 & 194 & 2000 & 3.8 & 4.6 & 4.1 & 3.1 \\
\hline E33 & 194 & 2000 & 3.9 & 2.5 & 4.4 & 3.3 \\
\hline E42 & 194 & 800 & 4 & 4.2 & 5 & 3.3 \\
\hline E14 & 136 & 800 & 4 & 3.3 & 5 & 3 \\
\hline E15 & 136 & 800 & 4.1 & 2.2 & 5 & 3.4 \\
\hline E16 & 136 & 800 & 4.2 & 4 & 5.6 & 4 \\
\hline D1 & 97 & 400 & 5 & 6 & 8.9 & 6.6 \\
\hline D2 & 97 & 400 & 5 & 8.3 & 8.9 & 6.6 \\
\hline E62 & 121 & 2000 & 6 & 13.2 & 12 & 11 \\
\hline E60 & 121 & 2000 & 6.2 & 11.4 & 12.8 & 11.4 \\
\hline E71 & 78 & 800 & 7.2 & 11.5 & 19.6 & 17 \\
\hline E72 & 78 & 800 & 7.8 & 16 & 20.7 & 19.8 \\
\hline
\end{tabular}

From Table 5.1, measured growth rates from five out of the thirteen shots fall in the window bounded by $\gamma_{30}$ and $\gamma_{\max }$, all of the growth rates fall within $50 \%$ of these theoretical upper and lower bounds, and all but two (shots E15 and E71) fall within 25\%. Considering (1) the sources of error, which include manufacturer specified $20 \%$ tolerance on the foil width, approximately $10 \%$ error on cutting the mm-scale foil thickness, and $\sim 10-20 \%$ frame-to-frame error on the camera response; (2) the inherent assumption that the background aluminum is expansionless and its properties can be extrapolated from (2.38); and (3) the large value of $\delta T / T$, which exceeded unity for all of these measurements, the close agreement with linear ETI theory is remarkable.

For negligible thermal conductivity losses, the growth rate of striation ETI is given by

$$
\gamma_{\max }=\frac{\partial \eta}{\partial T} \frac{J^{2}}{\rho c_{v}}
$$


Since all of the measurements were taken when the unperturbed foil was at approximately the same state (in biphase liquid/vapor), the material properties should be relatively constant, and the growth rate is expected to be proportional to $J^{2}$. Figure 5.23 shows the growth rates from Table 5.1 plotted as a function of current density.

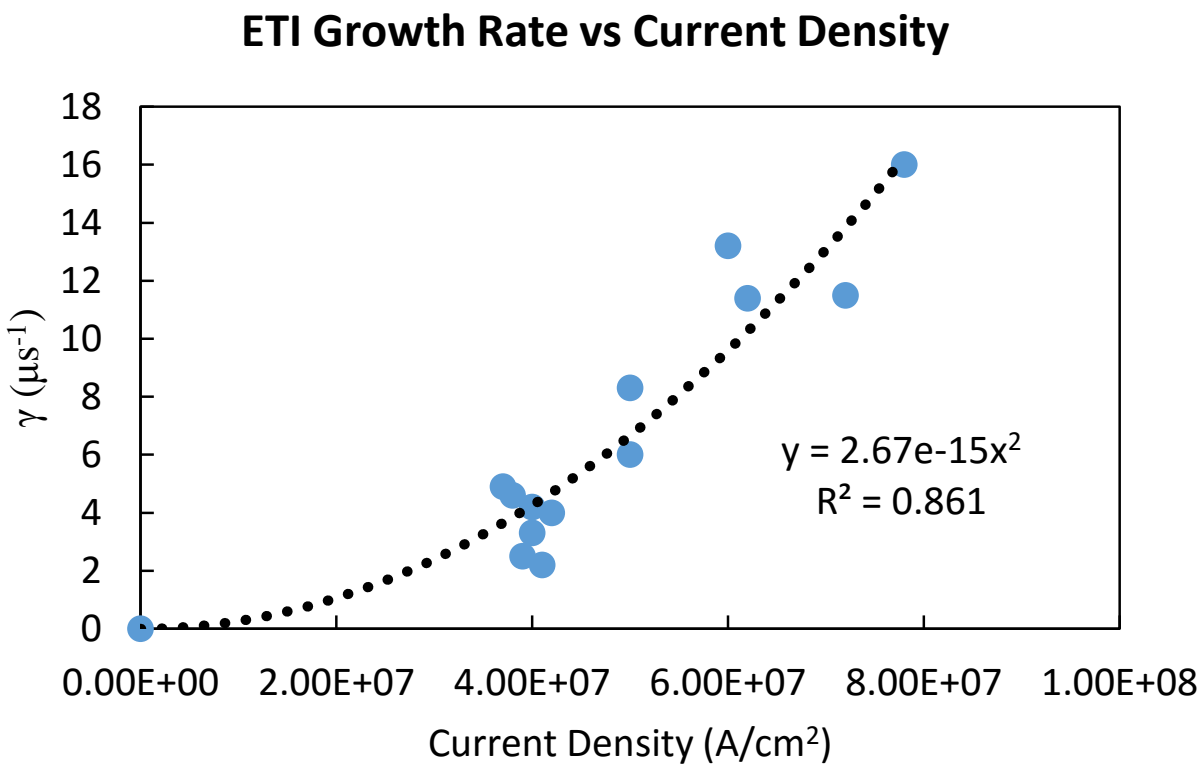

Figure 5.23: Experimentally measured growth rates from Table 5.1 plotted against current density. Results are consistent with quadratic dependence on current. Note that for large features with growth rate described by (5.9), the y-intercept should be approximately 0 (which has been enforced on the fit), so the data do not as strongly support a linear fit with this criterion.

For the shots analyzed, temperature perturbations were found to grow at a rate in good agreement with the rate predicted by linear ETI theory, and the growth rates were found to exhibit scaling consistent with predicted $J^{2}$ dependence. Collectively, this is strong evidence that the growing temperature perturbations observed on the ablating foils are in fact manifestations of the striation form of ETI. 


\subsection{Seeding of ETI}

While ETI has received recent attention $[6,11,23,24]$ as a proposed seeding mechanism for later-time plasma instabilities, the question of what seeds ETI itself remains open [62]. Studies at Sandia National Labs $[11,23]$ showed that surface finish is uncorrelated to ETI growth except in cases of surfaces in which roughness has been deliberately enhanced over normal values for metal finishes. The two mechanisms most often proposed as seeds of ETI are grain boundaries and inclusion of impurities. Recent work [62] on cylindrical rods has shown that ETI growth is substantially diminished in high purity (99.999\%) aluminum compared to alloy 6061 (99.1\%) aluminum in samples of identical surface finish, which indicates impurity inclusion may be more important than grain boundaries in seeding ETI.

Two experiments were performed to simulate the effects of grain boundaries and impurity inclusions on the planar foil ablation experimental setup. For the first experiment, a standard \#0-80 screw was rolled onto a sheet of $800 \mathrm{~nm}$ aluminum foil to impress deep grooves approximately $320 \mu \mathrm{m}$ apart. The grooves were impressed at a 70-degree angle relative to current flow to avoid confusing randomly occurring perpendicular-to-current structures with structures seeded by the grooves. A preshot image of the foil and the resulting temperature surface plot sequence are shown in Figure 5.24. 

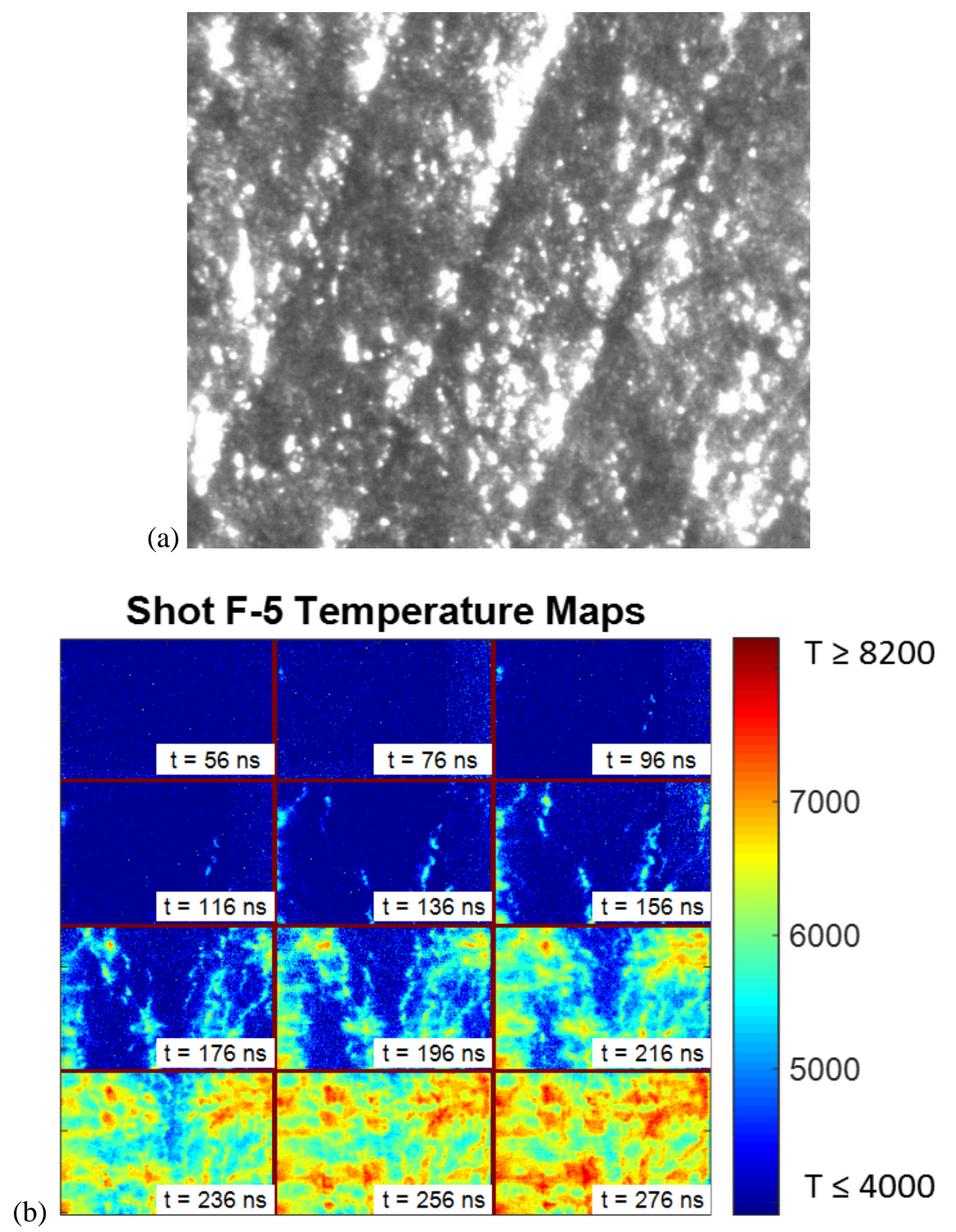

Figure 5.24: (a) Pre-shot image showing deep grooves embedded in an $800 \mathrm{~nm} \times 0.7 \mathrm{~cm} \times 0.9 \mathrm{~cm}$ foil, to approximate resistivity jumps at grain boundaries. Grooves are approximately $320 \mu \mathrm{m}$ apart. (b) Temperature plot sequence showing instability growth. All image sizes are $1.25 \mathrm{~mm}$ x $1.08 \mathrm{~mm}$; current flows from left to right.

The instability growth on Figure 5.24b shows little correlation with the deep grooves impressed into the foil, despite the fact that the wavelength of the grooves is large enough to 
grow ETI from the start of current. A second seeding experiment used two holes machined in the foil to simulate resistive material inclusions. The holes were positioned such that the line joining them was at the same 70-degree angle as the impressed grooves on the previous experiment. Because the primary impurity in 6061 aluminum is silicon, which has a room temperature resistivity that is 7 to 8 orders of magnitude higher than that of aluminum, the holes were taken to be a good approximation of a large silicon inclusion. Figure 5.25 shows results from a shot with the machined holes. 

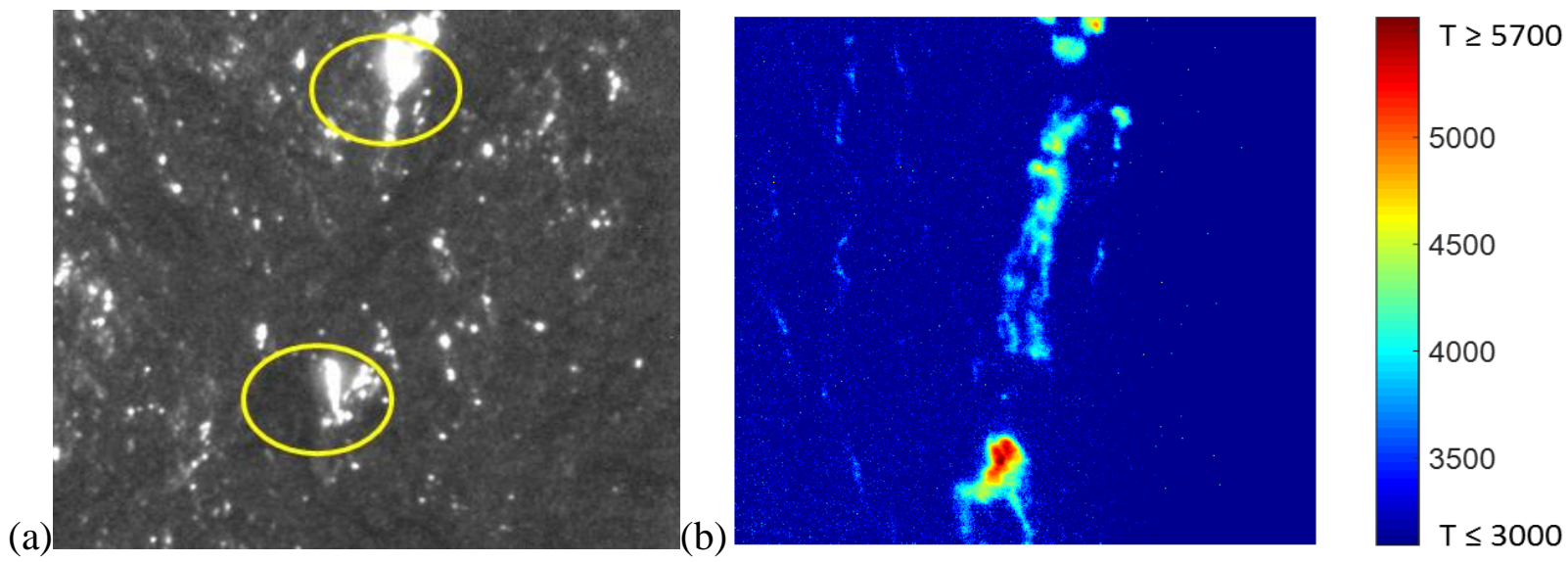

\section{Shot D-2 Temperature Plots}

(c)
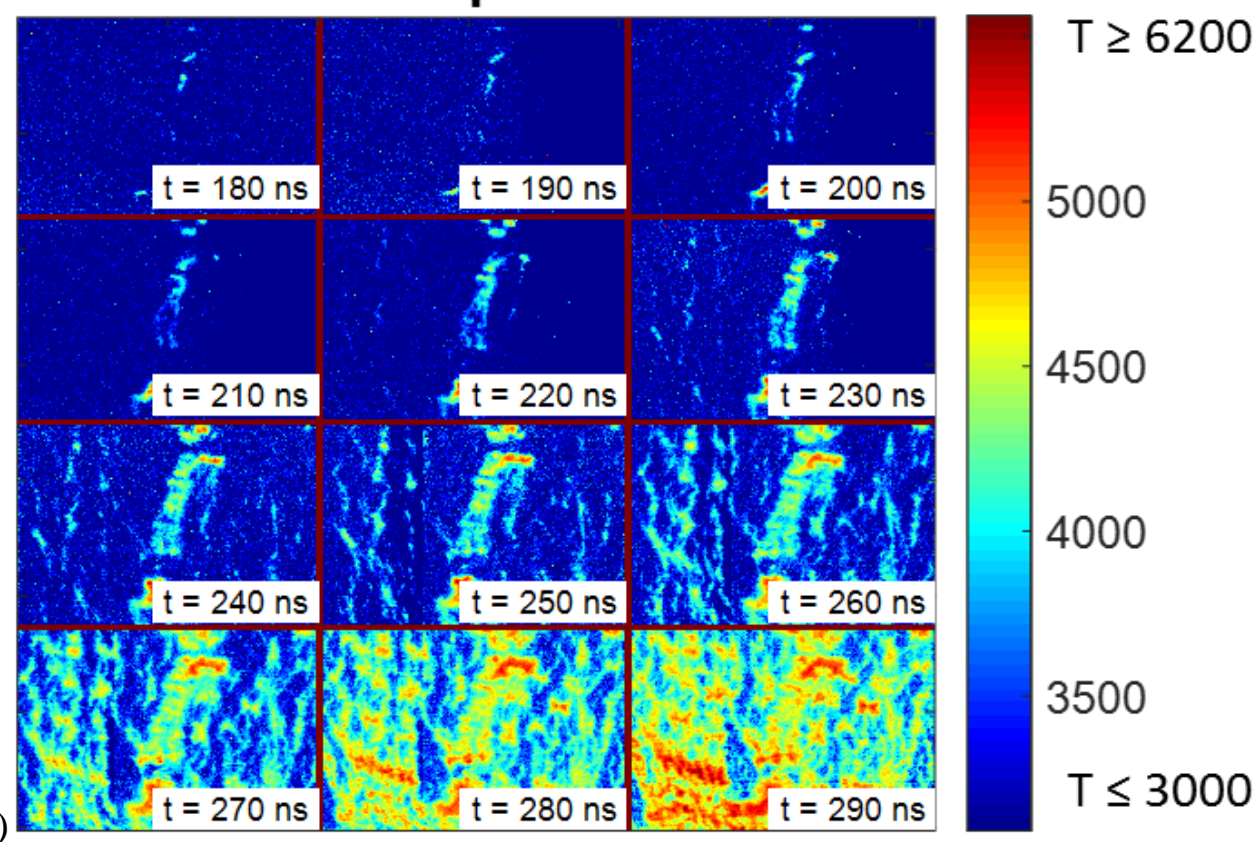

Figure 5.25: (a) Pre-shot image showing two holes with diameter $>100 \mu \mathrm{m}$ punctured in a $400 \mathrm{~nm} \times 1.0 \mathrm{~cm} \times 0.9$ $\mathrm{cm}$ foil, to approximate large areas of high resistivity. The hole locations are highlighted in the yellow circles (b) Temperature plot generated from image taken at 220 ns showing ETI preferentially forming along the line between the holes before growing elsewhere along the foil (c) Full shot temperature sequence. All image sizes are $2.5 \mathrm{~mm} x$ $2.15 \mathrm{~mm}$; current flows from left to right.

A clear ETI striation can be seen on Figure 5.25b, joining the position of the holes shown on Figure 5.25a. This provides experimental confirmation for the self-correlation of ETI around a resistive hotspot shown in Figure 2.4, which has previously been observed in simulation [62]. 
Figure $5.25 \mathrm{c}$ shows that the seeded structure is the first ETI striation to form and is dominant up until most of the striations have expanded and merged.

These experiments indicate that localized higher resistivity volumes in the foil are more likely to seed ETI than physical damage to the foil structure. Further evidence suggesting grain boundaries are unlikely to be responsible for seeding ETI can be found by inspecting Figure 2.12. For the early part of the current rise, the minimum growing wavelength is many 10 s of microns, and by the onset of melting is still around $20 \mu \mathrm{m}$. Grains of most metals are unlikely to be this large unless annealing has been performed to increase the grain size [72]. If the grain size of the aluminum foils actually were this large, grain boundaries would be visible on the $2 \mu \mathrm{m}-$ resolution optical magnification system used to image the foil ablations. Provided that grain size is in fact below the minimum growing wavelength, any perturbations seeded by grain boundaries will damp due to thermal conductivity, leading to homogenization of temperature by the time melting begins. This is further supported by theory [19] and experiment [20] showing that ETI does not significantly grow in the solid phase of most metal ablations. The available evidence points to impurity inclusions as the most significant contributor to the formation of ETI.

Nonuniformities in the material are random, and this likely explains the shot-to-shot variation in feature size observed on the temperature measurements described in Section 5.3. Nearby hotspots can merge together, as observed in Figure 5.25, to form striations, provided the angle between the line joining the hotspots and the current is larger than the minimum growing angle for striation-form ETI. This angle approaches 45 degrees ( $\pi / 2$ radians) for large perturbations, although angles closer to 90 degrees will exhibit higher growth rate. The relative growth of striations of different wavelengths and angles as a function of the size or magnitude of resistivity perturbations would be an interesting computational study for future work. 


\subsection{Formation of Filamentation ETI}

The majority of this work has focused on the growth of striation-form ETI. Hot

structures parallel to the direction of current, believed to be the filamentation form of ETI, were also observed on several shots that included late-time images after plasma features had sufficient time to merge and form plasma channels. Figure 5.26 shows a typical temperature surface plot sequence on which this phenomenon was observed.
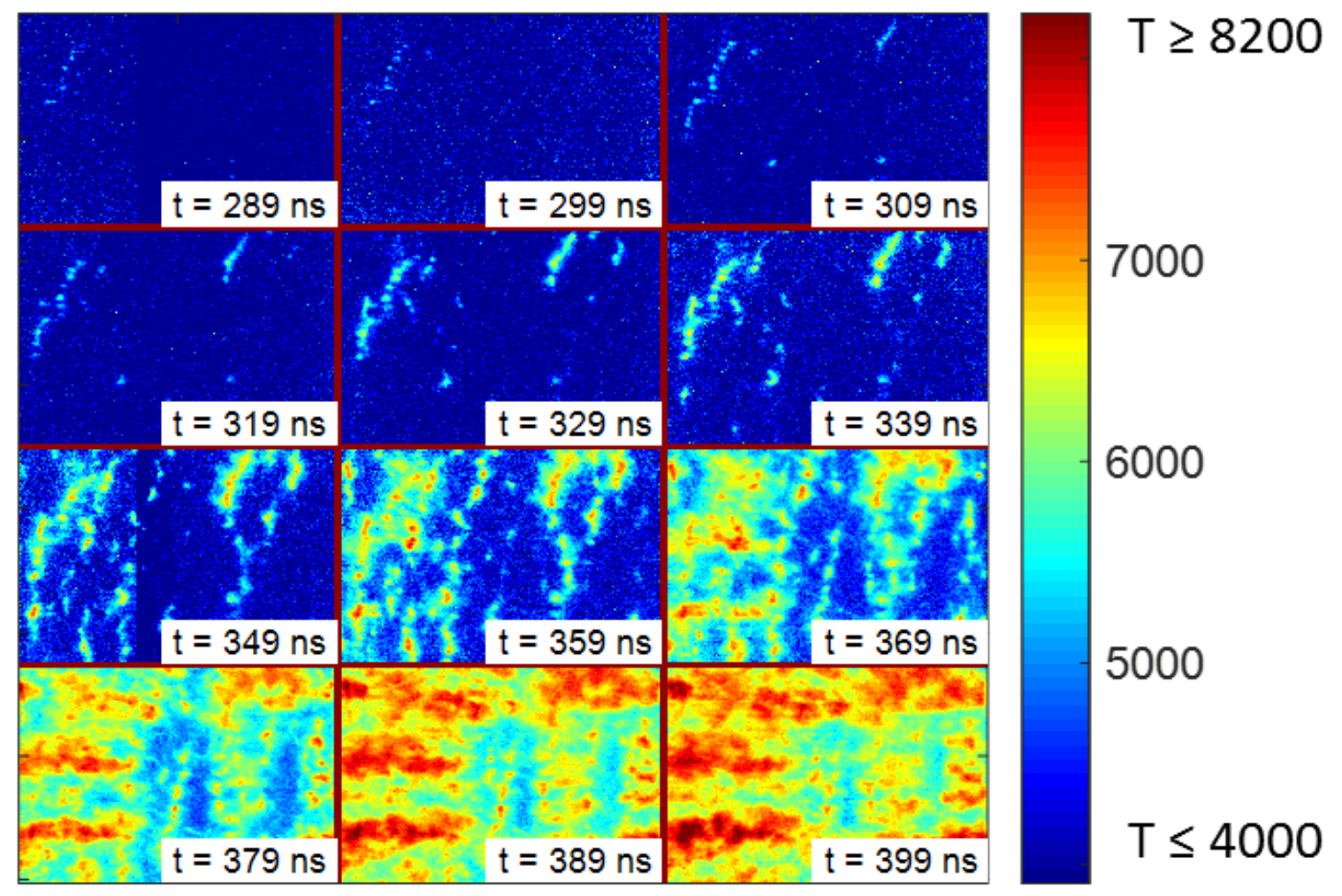

Figure 5.26: Temperature surface plot of an $800 \mathrm{~nm} \times 0.7 \mathrm{~cm} \times 0.9 \mathrm{~cm}$ aluminum foil ablation showing plasma from vaporized sections of the foil joining to form filamentary structures late in time.

Figure 5.26 appears to show the transition from striation ETI to filamentation ETI once the hot aluminum gas has sufficiently ionized such that $\frac{\partial \eta}{\partial T}$ changes signs. Determining a theoretical growth rate for this instability is significantly more involved than for the striation form of ETI because the unperturbed material is a dense, weakly ionized aluminum plasma with 
strongly ionized, hot filaments As the purpose of the work presented in this dissertation was to analyze the growth of striation-form ETI as it is relevant to seeding of perpendicular-to-current instabilities on liners, such as the MRT instability, analysis of the filamentation ETI data has been left as a suggestion for future work. 


\section{CHAPTER 6}

\section{Seeding of Hydrodynamic Instabilities from the Electrothermal Instability on Liner Ablation Experiments Driven by a MA LTD}

The previous chapter focused on the growth of striation-form ETI as a temperature perturbation, which occurs in the solid, liquid, and gas phases (and continues for some time into the plasma phase due to the density dependence of resistivity). However, it is the mass perturbation resulting from portions of a liner ablating faster than the bulk material that is of greater concern, as this process perturbs the plasma-vacuum interface. The interface perturbation provides an initial seed for destructive hydrodynamic instabilities, including kink, sausage, and, most importantly, MRT, which has been identified as the most significant obstacle to the MagLIF concept [73]. This chapter presents results from experiments performed on MAIZE to investigate the growth of sausage and MRT instabilities on liners where the conditions for ETI development have been deliberately altered.

\section{1: Material Dependence of Instability Growth}

The growth rates of ETI discussed in Chapter 2 depend on material-specific properties, including electrical resistivity and its derivative with temperature, density, and thermal conductivity. Therefore, it is anticipated that measurable changes in the seeding of plasma instabilities should occur for materials that differ in these values. To investigate these effects, shots were performed on MAIZE using liner loads fabricated from aluminum, tantalum, ultrapolyester coated aluminum, and ultra-polyester coated titanium, as described in Chapter 3. 
A typical current trace from a liner shot is shown in Figure 6.1. Regardless of material, all liner loads exhibited values of time-averaged resistance between 0.2 and 0.3 ohms and timeaveraged inductance between 22 and $23 \mathrm{nH}$. This corresponds to an anticipated peak current of $550 \mathrm{kA}$ and a risetime of $230 \mathrm{~ns}$. None of the shots differed from these values of peak current and risetime by more than $5 \%$.

\section{Shot 1173 Current Trace}

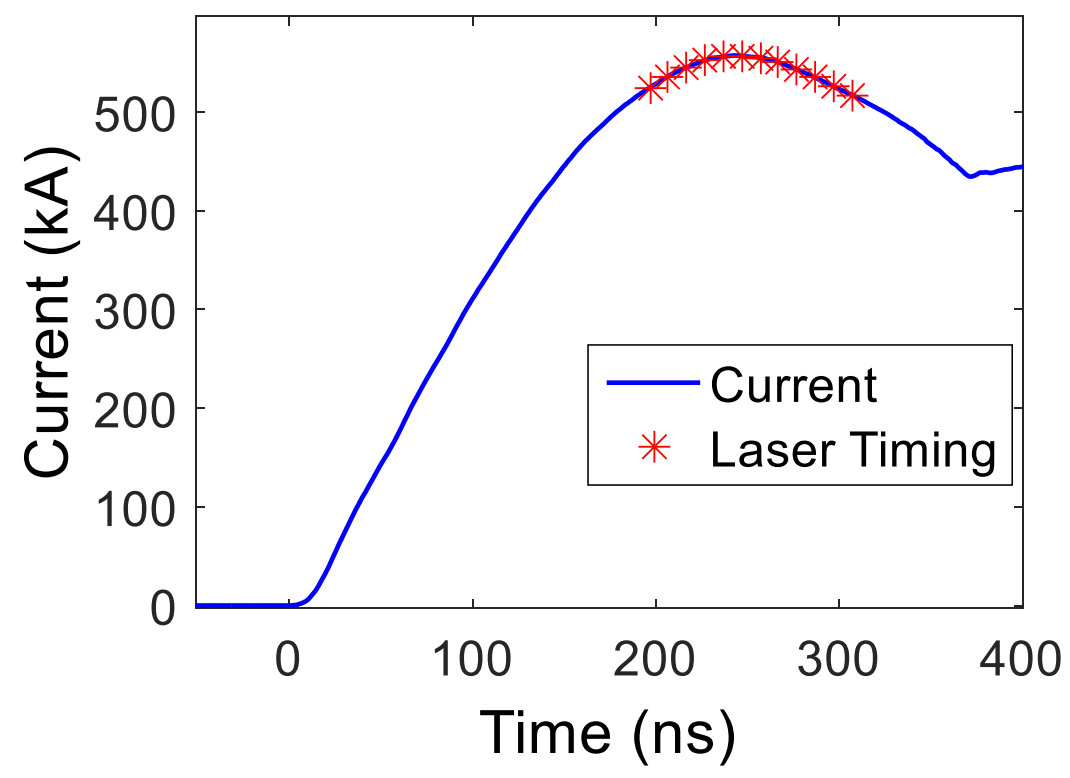

Figure 6.1: Typical current trace with shadowgraph/self-emission image timings indicated on the figure. The 557 kA peak current and $240 \mathrm{~ns}$ risetime were characteristic of liner load shots.

Data from the combined shadowgraphy-self emission diagnostic discussed in Chapter 3 were used to obtain 12-frame sequences of plasma-vacuum interface location. After several shots, it was discovered that the laser backlighting was inconsequential to obtaining the interface position, and as a result several of the later shots did not use the laser backlighter, imaging only self-emission at $532 \mathrm{~nm}$. Representative image sequences for bare aluminum, ultra-polyester backed aluminum, and ultra-polyester backed titanium are shown in Figures 6.2 through 6.4, 
taken at comparable imaging times centered around peak current (as indicated in Figure 6.1). All of these liners utilized the dumbbell support structure described in Chapter 3 and in Ref [37].
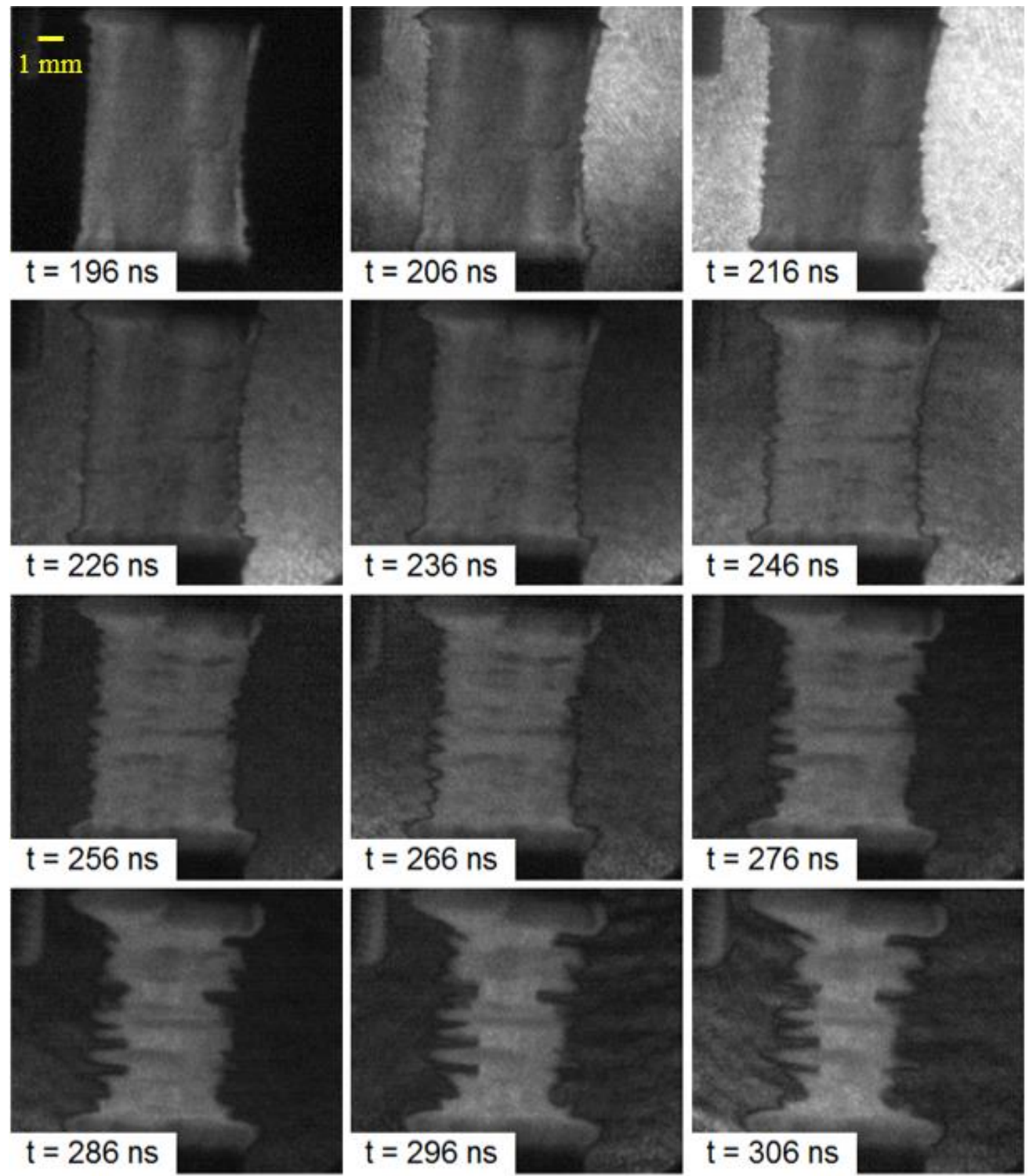

Figure 6.2: Image sequence of a $400 \mathrm{~nm}$ bare aluminum liner showing well-developed MRT coupled to the sausage mode. These liners had the lowest linear mass of all liners fielded and exhibited the most dramatic implosion. 


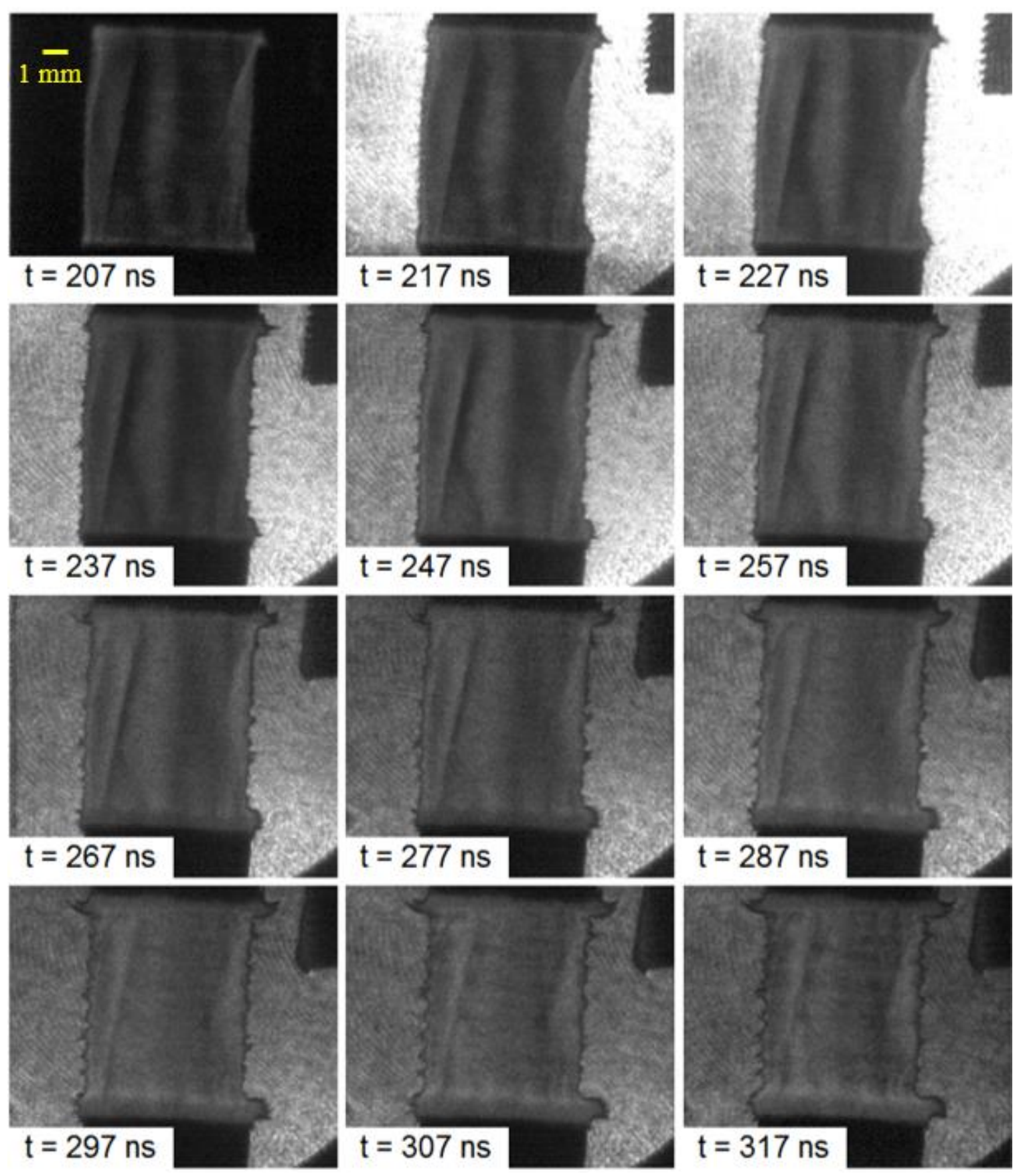

Figure 6.3: Image sequence of a $200 \mathrm{~nm}$ aluminum liner with a $1.5 \mu \mathrm{m}$ ultra-polyester coating on the inner surface. The higher linear mass of these liners compared to the bare aluminum resulted in lower acceleration and less MRT growth. 

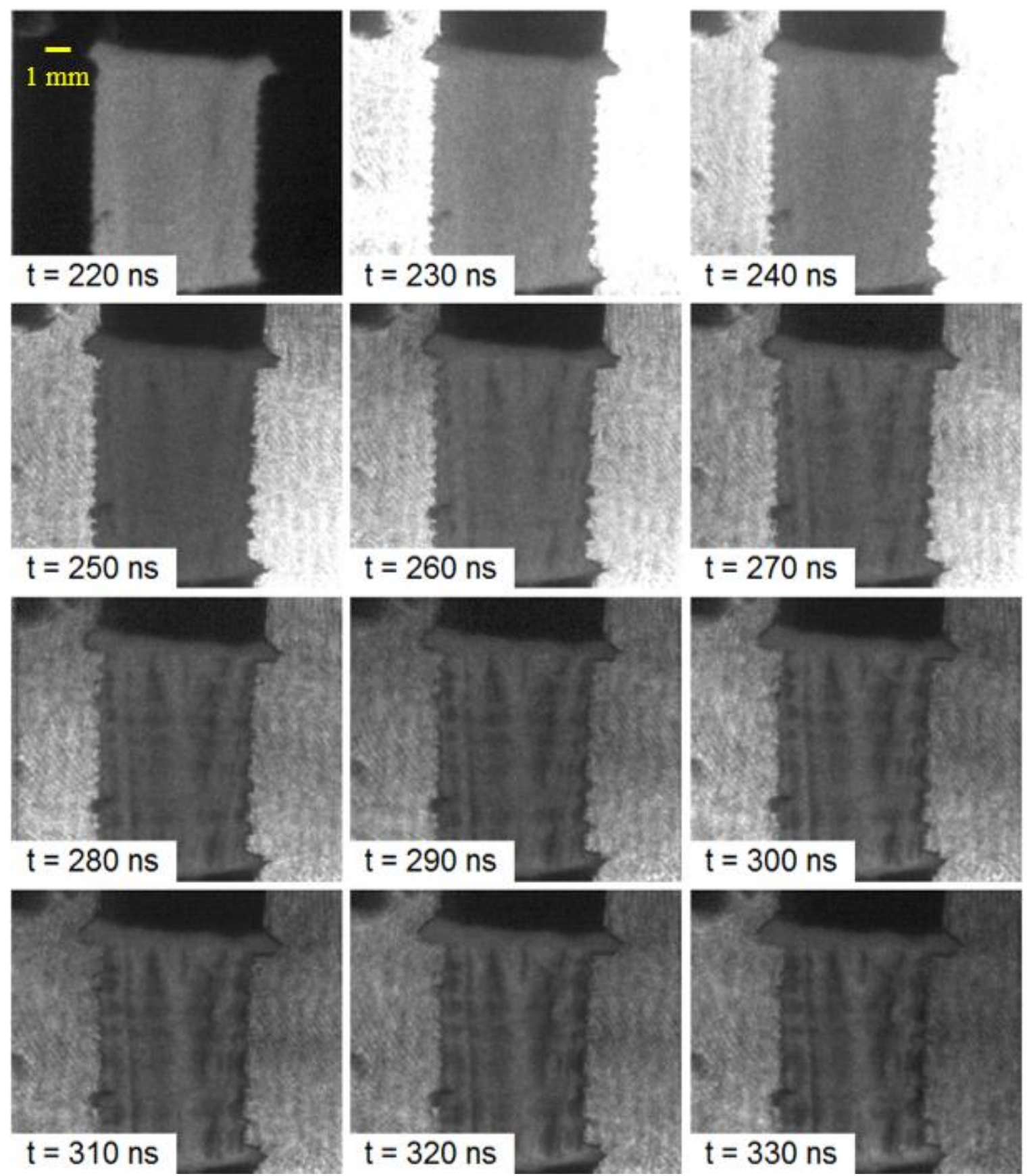

Figure 6.4: Image sequence of a $250 \mathrm{~nm}$ titanium liner with a $1.5 \mu \mathrm{m}$ ultra-polyester coating on the inner surface. This liner has the highest linear mass that has been observed to exhibit implosion on MAIZE, although the amount of inward motion of the plasma-vacuum interface is minimal.

For each shadowgraph figure obtained, the image is rotated to align the direction of current with the y-axis. Distance from the center of the initial liner (from the pre-shot image) is 
extracted from a fit of the two plasma-vacuum interfaces as a function of height, over a defined region of interest to exclude the edge effects clearly occurring at the top and bottom of the implosion region. Taking an average value of one of these interface functions gives the radius of the plasma at a specific time. Applying a Fourier transform to the interface function extracts the wavelength components of instability structures, and the function

$$
A=\frac{1}{\sqrt{2}}\left(\operatorname{Stddev}\left(\Delta \mathrm{x}_{\text {right }}\right)+\operatorname{Stddev}\left(\Delta \mathrm{x}_{\text {left }}\right)\right)
$$

defines an instability amplitude, where $\Delta \mathrm{x}_{\text {right }}$ and $\Delta \mathrm{x}_{\text {left }}$ are the left and right interface functions, respectively, and perturbations are assumed to be sinusoidal in nature. Figure 6.5a shows a sample interface fit along with its associated interface perturbation functions and their Fourier transforms. 
(a)
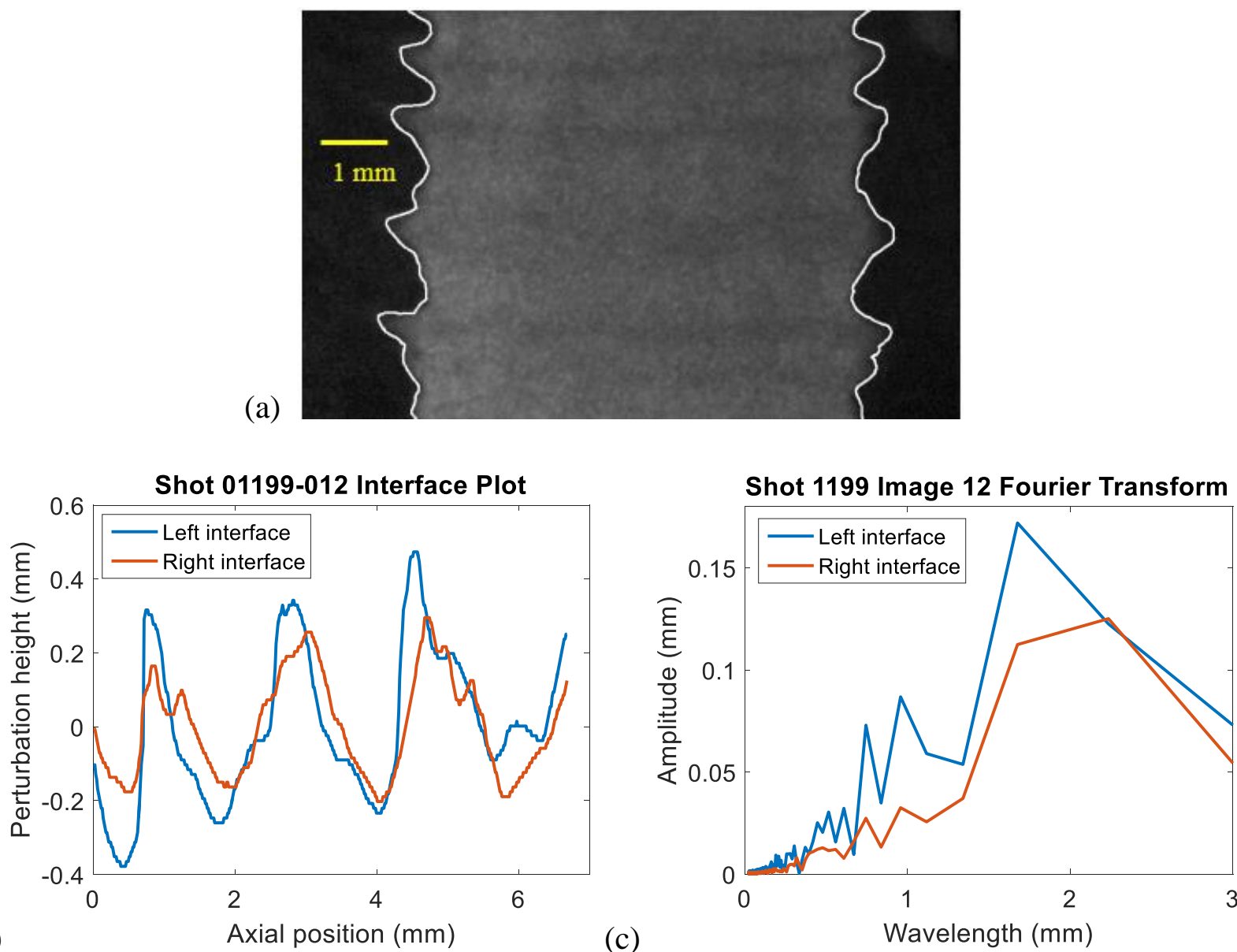

Figure 6.5: Interface analysis of a region of interest of an ultra-polyester backed $400 \mathrm{~nm}$ aluminum liner region of interest at $400 \mathrm{~ns}$. (a) Interface fit (b) Interface perturbation functions for the left and right plasma-vacuum interfaces on Figure 6.5a. The RMS amplitude of these perturbations calculated from (6.1) is $0.28 \mathrm{~mm}$ for the left interface and $0.19 \mathrm{~mm}$ for the right interface (c) Fourier transforms of the perturbation functions.

This procedure was used to analyze growth of plasma instabilities in two case studies, which are described in the following sections.

\subsection{1: MRT Instability on Plastic-Backed Aluminum and Titanium Foils}

Equal mass samples of ultra-polyester coated aluminum and titanium foils were prepared using the deposition technique described in Chapter 3. Foil thicknesses were $200 \mathrm{~nm}$ (aluminum) and $125 \mathrm{~nm}$ (titanium); in the liner geometry, the foils had a total linear mass density of $0.41 \mathrm{mg} / \mathrm{cm}$. Ablations were imaged from $\sim 100$ to $350 \mathrm{~ns}$ over several shots. These materials 
were chosen because titanium has a resistivity that is much greater than aluminum (by about a factor of 20) over a large temperature range $[26,74]$ with a density and specific heat capacity that differs by no more than a factor of 2 [75].

Results from these experiments are shown in Figures 6.6 and 6.7. Figure 6.6 presents wavelength spectra for the two materials taken at various times. Two shots were used for each material to provide a 200 window for instability analysis. While the titanium shots show slightly greater interface perturbation early in time, and also slightly more long-wavelength component growth later in time, the overall instability wavelength spectra are fairly similar, particularly given the large $(>10 x)$ difference in material properties. Similar observations can be made from Figure 6.7. While the growth rate appears to be somewhat larger later in time for titanium, the early time instability amplitude is nearly identical for the two materials. ETI is much more relevant to the early-time features, as it is a seed for the observed plasma instabilities. However, striation ETI is unlikely to impact the late-time growth, as the material has become a plasma well before the divergence in amplitude is observed on Figure 6.7. 
Instability Fourier Transform, $\mathbf{t} \approx \mathbf{1 2 0}$ ns

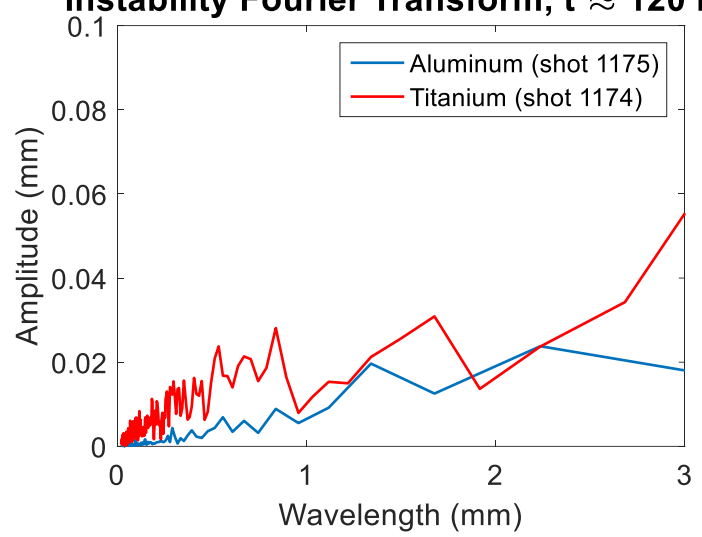

(a)

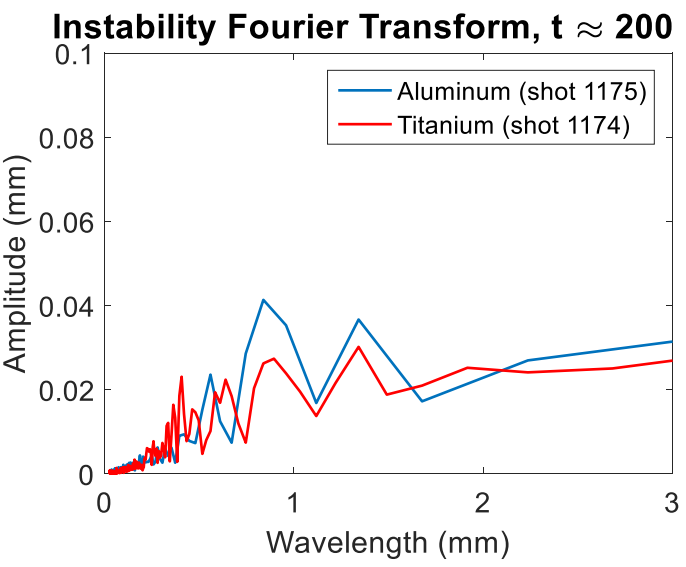

(c)

Wavelength $(\mathrm{mm})$

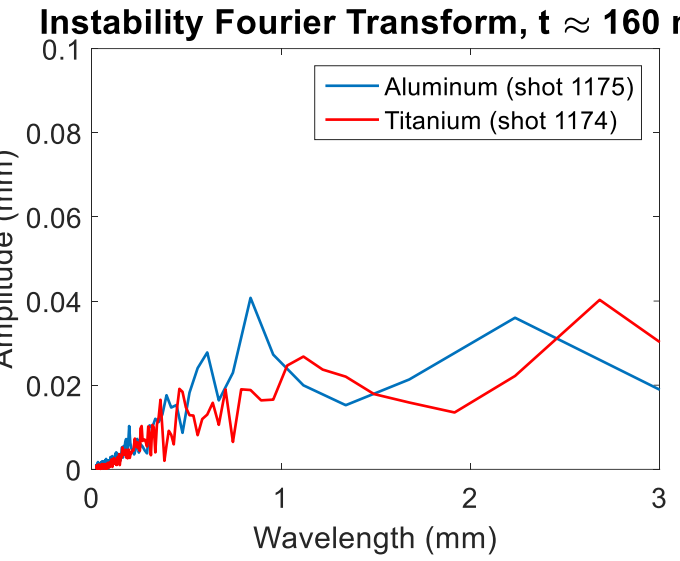

(b)

Instability Fourier Transform, $\mathbf{t} \approx \mathbf{2 4 0} \mathrm{ns}$

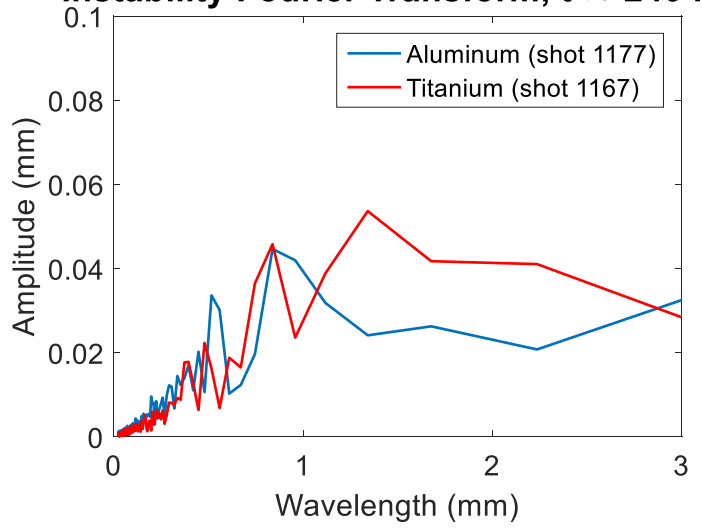

(d)

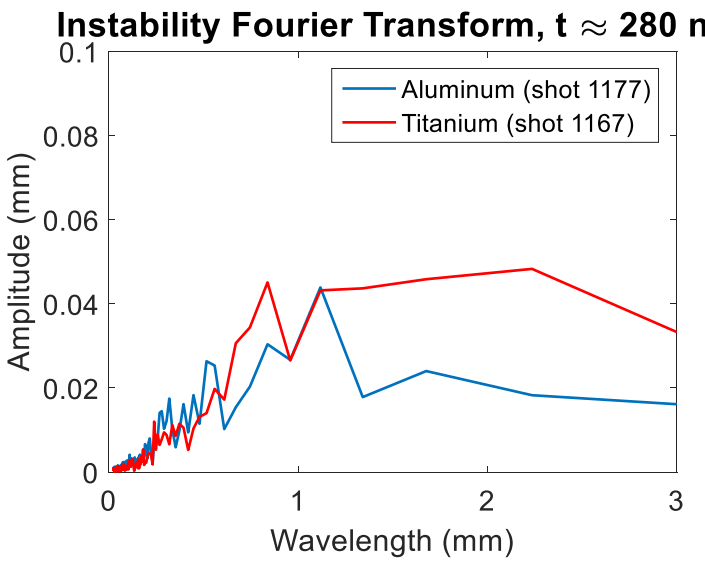

Figure 6.6: Fourier transforms of plasma-vacuum interfaces for aluminum and titanium liners at times from 120 to $280 \mathrm{~ns}$ after start of current. While the titanium liners show slightly more growth of long-wavelength features, overall both materials exhibit similar wavelength spectra. 


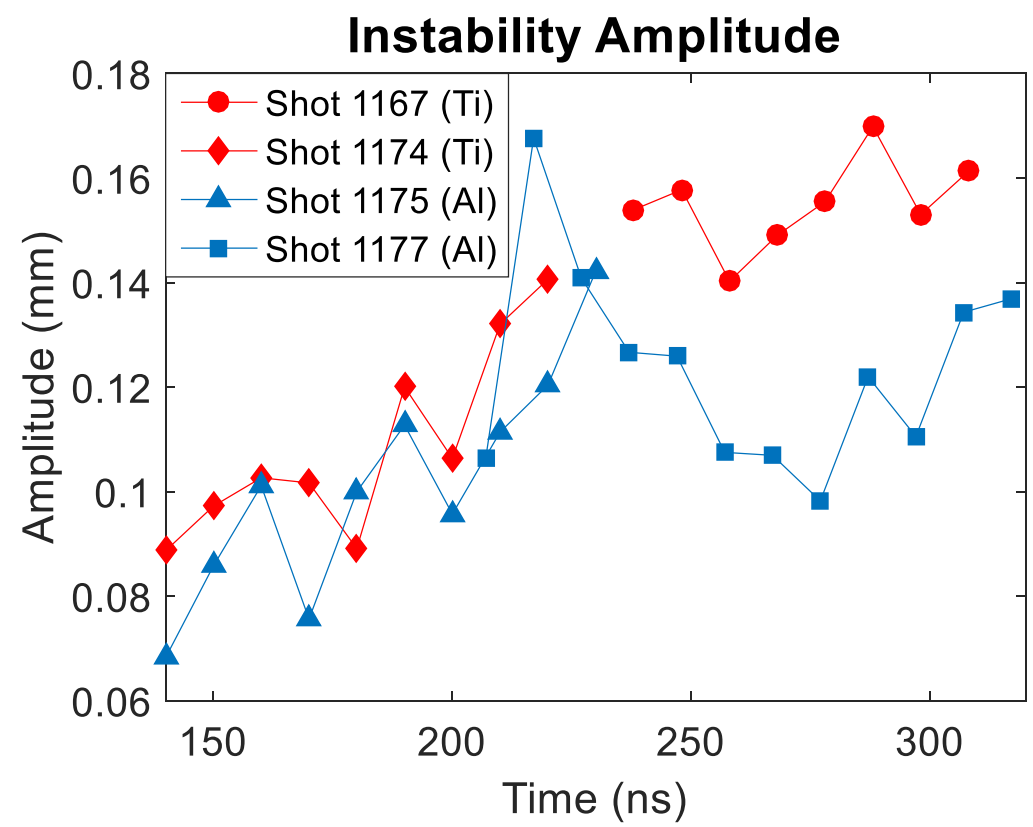

Figure 6.7: Instability amplitude determined from (6.1) as a function of time for aluminum and titanium liners. The growth is similar for the two metals.

It appears that instability seeding due to ETI on aluminum and titanium is roughly equivalent. This may be explained by the theory presented by Oreshkin [19], which states that for similar current drives, the most important parameter for determining the integral growth of ETI is the ratio of the critical temperature to the melting temperature, and that the integral growth can be approximated by

$$
\Gamma=\ln \left(\frac{T_{\text {crit }}}{T_{\text {melt }}}\right)
$$

where $T_{\text {crit }}$ is the temperature at the critical point (the point at which a phase change from liquid to vapor does not require latent heat). For aluminum, the values of $T_{\text {crit }}$ and $T_{\text {melt }}$ are $8000 \mathrm{~K}$ [75] and $933 \mathrm{~K}$, respectively; these values are $15500 \mathrm{~K}$ [76] and $1941 \mathrm{~K}$. The ratios are therefore very similar; 8.6 for alumimum and 8.0 for titanium. 


\subsection{2: Sausage Instability on Aluminum and Tantalum Foils}

If (6.2) is a good indicator of the total growth of ETI, the largest reduction in seeding of plasma instabilities should result for a material with a low ratio of $T_{\text {crit }}$ to $T_{\text {melt }}$. One such material is tantalum, which has a critical temperature of $10250 \mathrm{~K}$ [76] and a melting temperature of $3293 \mathrm{~K}$. Liner ablations were performed on the solid plastic support structures to investigate the growth of the sausage instability [43] on a $500 \mathrm{~nm}$ tantalum liner compared to a typical 400 nm aluminum liner. Figures 6.8 and 6.9 show the framing camera images from these shots. 

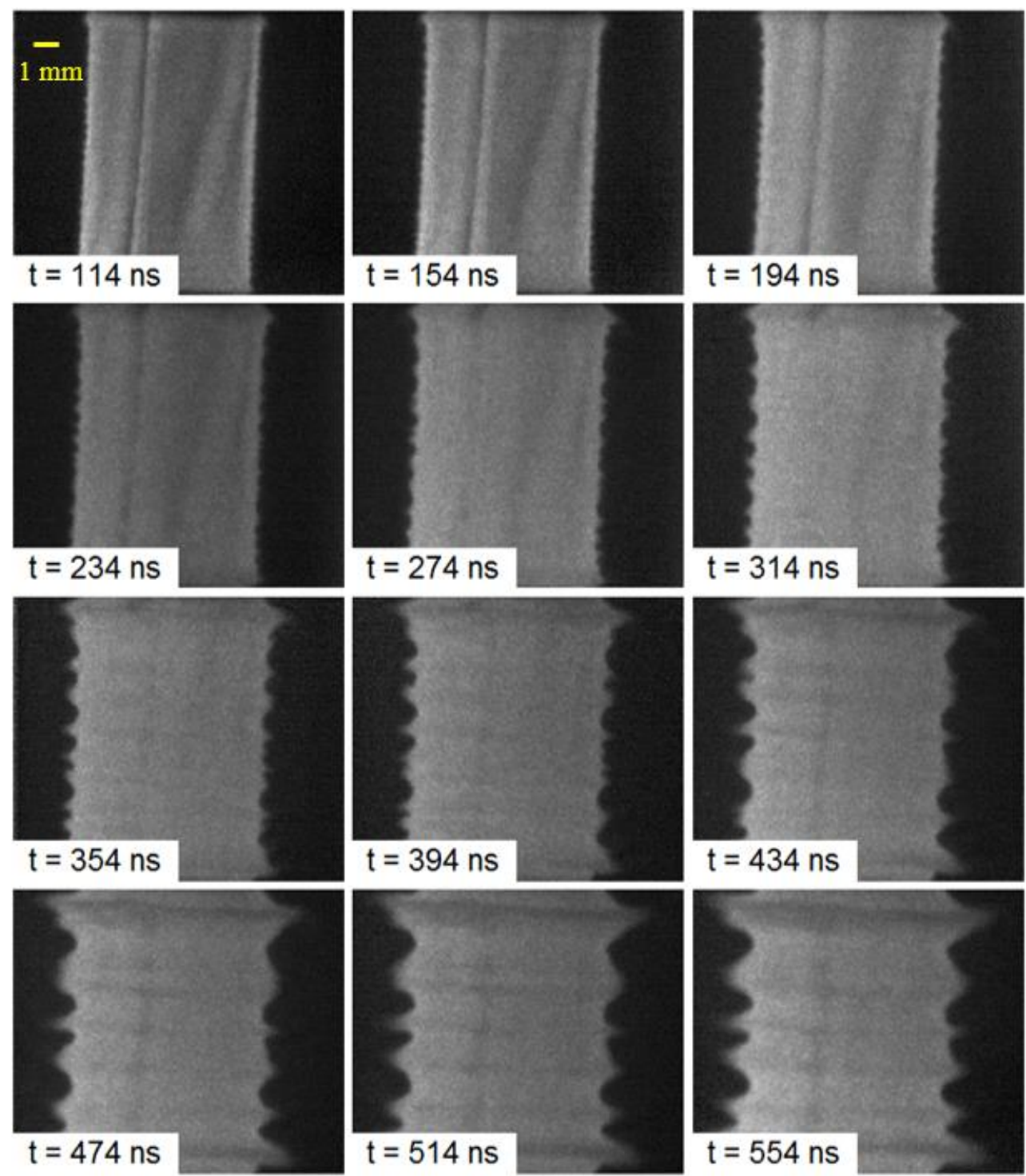

Figure 6.8: $400 \mathrm{~nm}$ aluminum liner ablation on solid plastic support; a lower frame rate was used to capture the evolution of the sausage mode instability over several hundred ns 

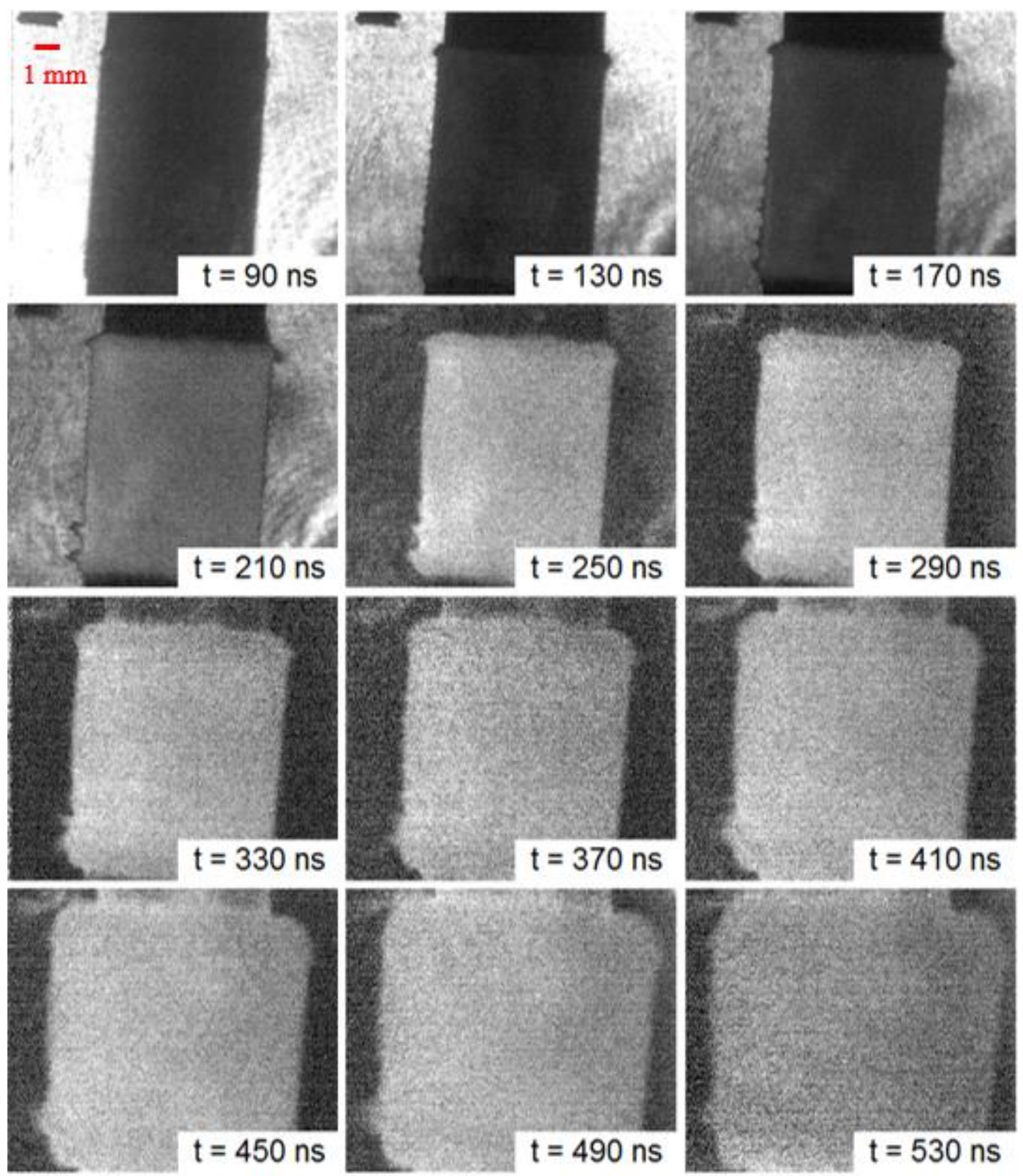

Figure 6.9: $500 \mathrm{~nm}$ tantalum liner ablation on solid plastic support imaged using the same frame rate as the aluminum liner in Figure 6.8. The plasma-vacuum interfaces are much more stable than in Figure 6.8. A small sausage instability structure believed to be seeded by edge effects can be seen on the bottom left of the liner interface, demonstrating that sausage mode can grow in this system when it is seeded. Later images were contrastenhanced to make the interface visible. 
Figure 6.9 shows a remarkable reduction in sausage instability growth compared to the aluminum liner shown in Figure 6.8. While the sausage instability is expected to grow less for the denser tantalum liner than for aluminum, since sausage instability grows as $1 / \sqrt{\rho}[39,43]$, the almost completely flat outer surfaces in Figure 6.9 imply the sausage instability was not seeded at all along most of the liner. Additionally, a few perturbations are visible along the bottom left side of the liner likely seeded by edge effects where the foil makes electrical contact with the support structure; these grow and merge similarly to the instability structures seen in Figure 6.8, indicating that the sausage instability can still grow on this ablation if seeded. One alternative explanation for the lack of instability formation is the stabilizing effect of outward acceleration (MRT with the opposite sign as the exponentially growing dependence on an inward accelerating liner). To eliminate this possibility, a tantalum liner was ablated on a dumbbell support structure. While the heavy tantalum had a linear mass density too high to implode on MAIZE, the lack of material pressure from the solid support structure greatly reduced the outward acceleration compared to the shot shown in Figure 6.9. Images from a dumbbell support shot are shown in Figure 6.10. 


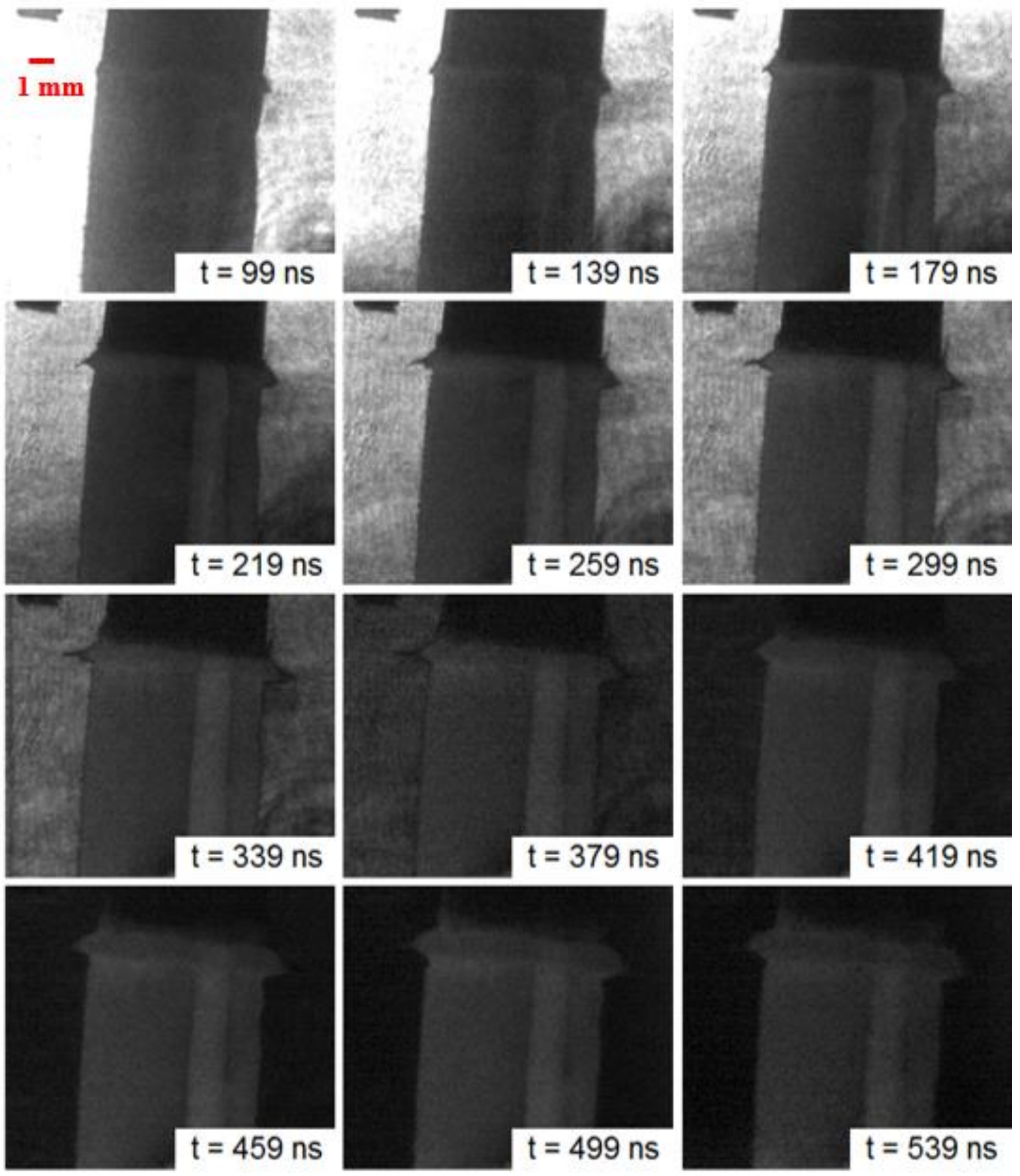

Figure 6.10: $500 \mathrm{~nm}$ tantalum liner ablation on dumbbell plastic support imaged at similar times as the liner in Figure 6.9a. The nearly static plasma-vacuum interface in this shot rules out the stabilizing effect of acceleration in the radially outward direction. Very little instability growth is observed.

Figure 6.10 shows a similar suppression of instability growth as Figure 6.9. The amplitude vs time data from Figure 6.8 through 6.10 are summarized in Figure 6.11. 


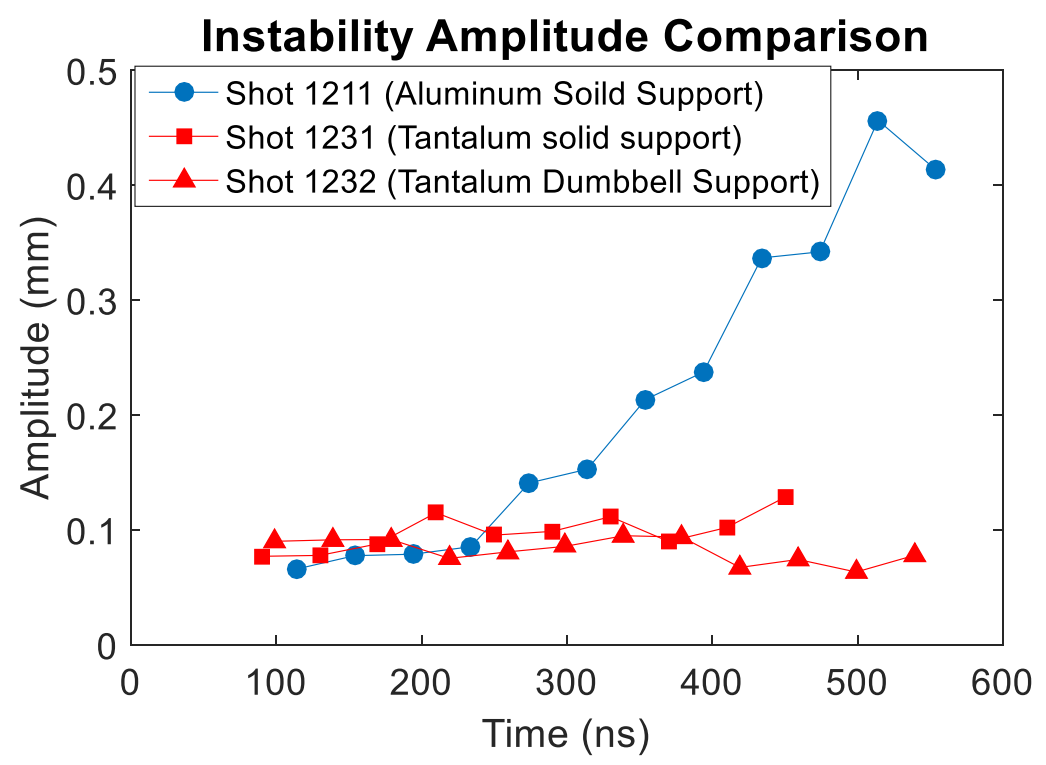

Figure 6.11: Instability amplitude vs time for the shots depicted in Figures 6.8 and 6.9. While instability grows exponentially for the aluminum liner, the amplitude of instabilities on the tantalum liners remain on the order of the imaging resolution for over $400 \mathrm{~ns}$.

Figure 6.11 confirms the qualitative observations from the framing camera image sequences. The aluminum liner exhibits an instability structure that grows exponentially in time, while the tantalum liners show little, if any, growth, with amplitude that is on the order of the imaging resolution of the system. This supports the claim that ETI seeds the sausage mode instability observed on solid liner MAIZE shots [43], as substantially less ETI growth is expected for the tantalum liners than for the aluminum liners.

\section{2: Effects of Dielectric Coating on the Sausage Instability}

In concert with results from the previous section, ETI has been proposed as the seeding mechanism for the sausage instability observed on exploding liners on MAIZE. This $\mathrm{m}=0$ instability has been consistently observed to grow on unseeded liners that use the solid support structure, despite the growth rate of competing $\mathrm{m}>0$ modes having comparable (and actually slightly greater) growth rates. When these alternative modes are seeded (either using a physical 
impression on the foil or by applying a magnetic field) growth is observed, and the plasma develops a kink or helical structure [43]. The preferential growth of $\mathrm{m}=0$ modes when no external seeding is applied is understood to be a consequence of the self-azimuthal correlation behavior of ETI. To obtain additional verification of ETI as the mechanism behind the seeding of azimuthally correlated instabilities, several liner explosions were conducted to compare the standard aluminum liners to aluminum liners with dielectric coatings on the external surface. These liners were fabricated from the ultra-Polyester backed foils described in Chapter 3. Coating a liner with a dielectric has been experimentally shown $[6,11]$ to inhibit the growth of ETI. Because adding a dielectric coating increased the total mass of the liner, additional ablations were conducted using liners with the dielectric coating present, but on the internal surface, to avoid confusing effects due to the applied coating with effects due to the increased mass. Figures 6.12 through 6.14 show typical framing camera image sequences from an uncoated liner, a coated liner with the dielectric on the internal surface, a coated liner with the dielectric on the external surface, respectively. 


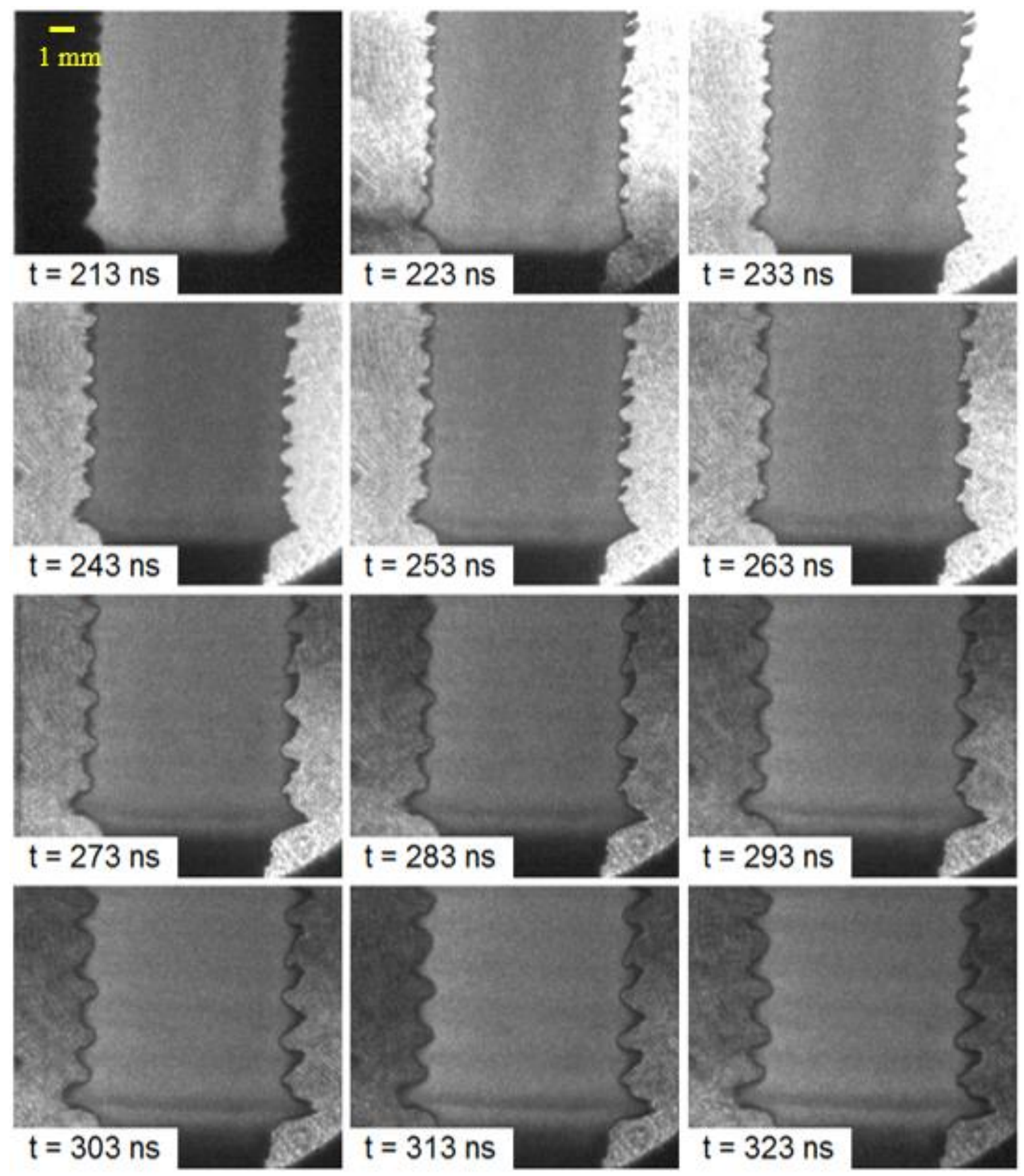

Figure 6.12: Shadowgraph sequence of exploding $\mathrm{Al}$ liner $(400 \mathrm{~nm})$ with no coating 


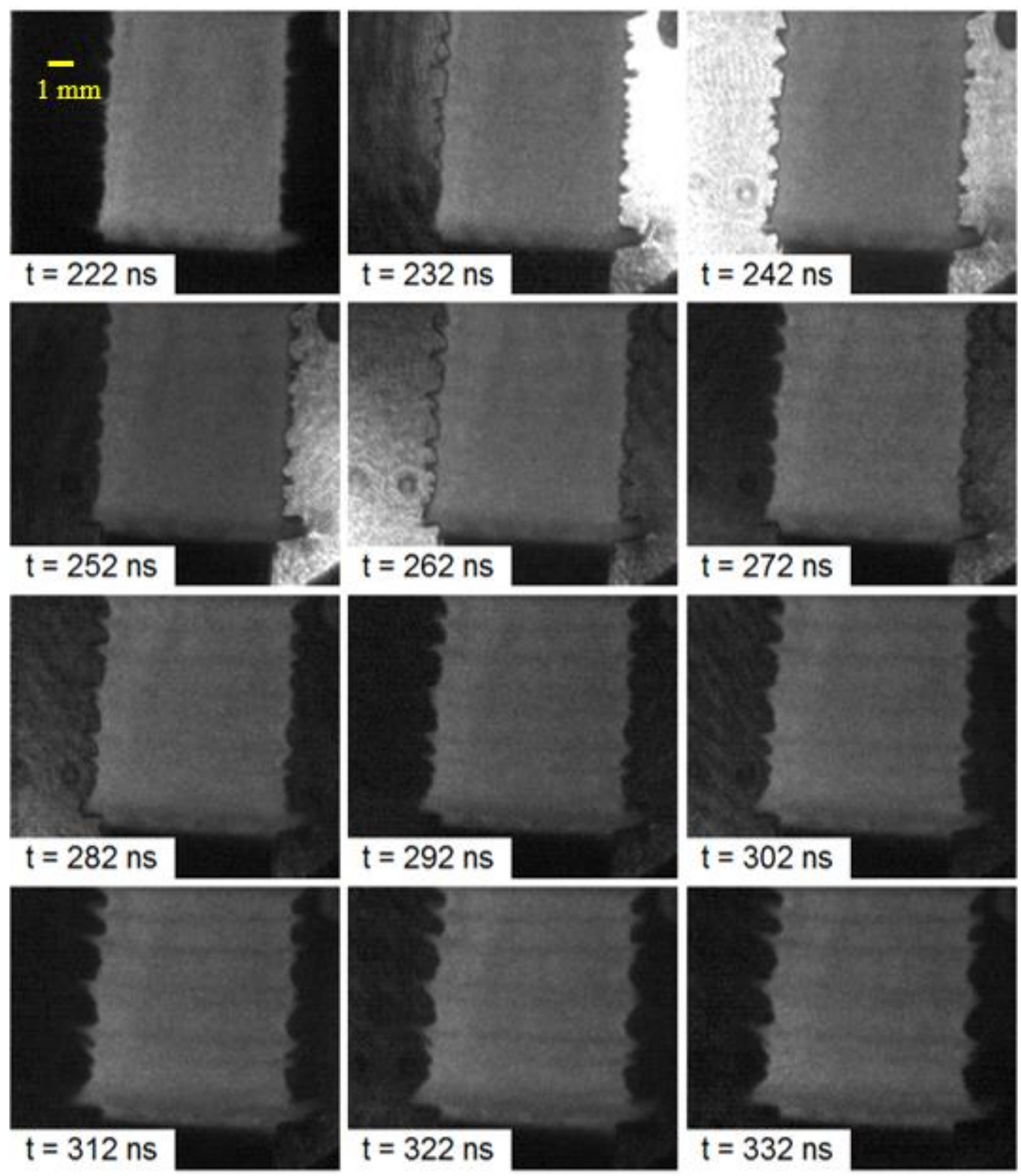

Figure 6.13: Shadowgraph sequence of exploding Al liner (400 nm) with ultra-polyester coating on inside 

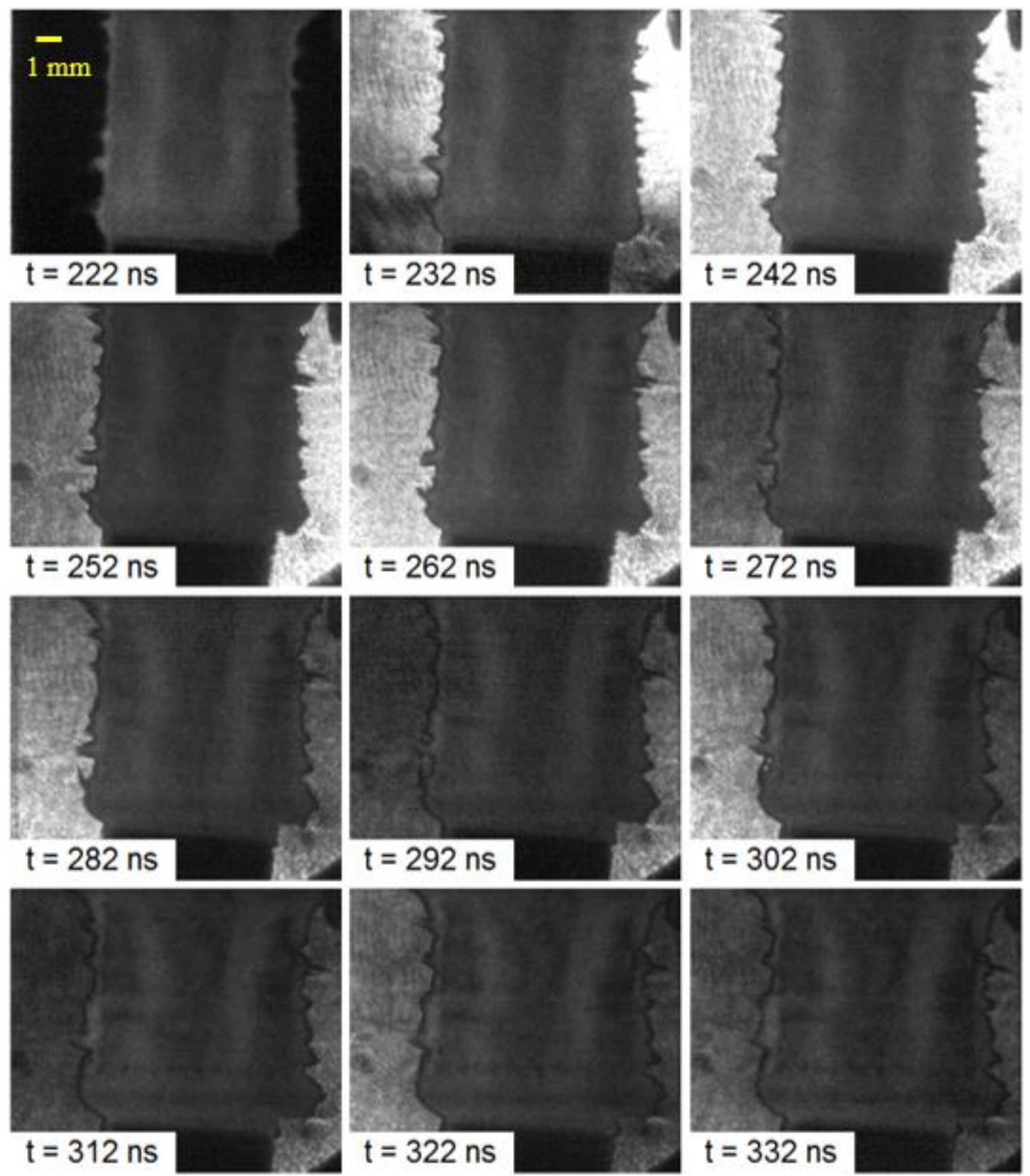

Figure 6.14: Shadowgraph sequence of exploding Al liner (400 nm) with ultra-polyester coating on outside

Figures 6.12 and 6.13 show qualitatively similar features: azimuthally correlated instability structures form and coalesce into longer-wavelength structures as time progresses. The features are comparatively smaller in amplitude on Figure 6.13, which is most likely a 
consequence of the additional mass of the plastic coating slowing down the sausage instability growth. On Figure 6.14, which shows the plastic-on-outside case, the azimuthal symmetry present in the uncoated and plastic-on-inside cases is not observed.

On Figures 6.12 and 6.13, the azimuthally correlated "bump" features are connected by horizontal dark bands in the self-emission of the foil, which are understood to be fully symmetric regions of expansion relative to the unperturbed plasma radius. These dark bands are also present on Figure 6.14, but they are not exclusively horizontal, and they generally do not connect bumps on the interface. These features are highlighted on the contrast-enhanced images shown in Figure 6.15, which are taken at similar times from Figures 6.12 through 6.14.

(a)

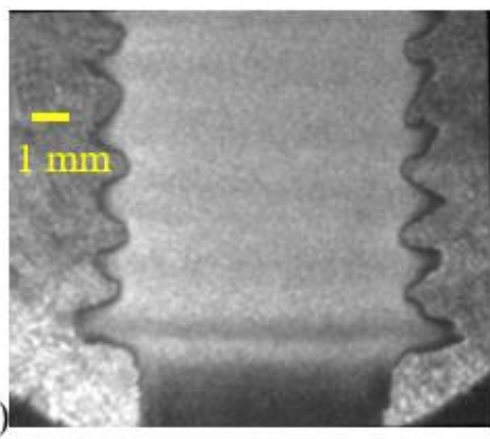

(d)

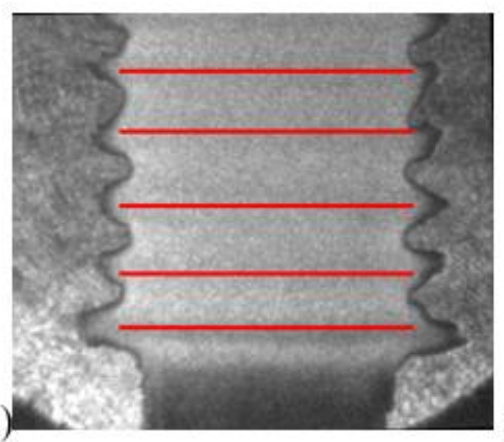

(b)
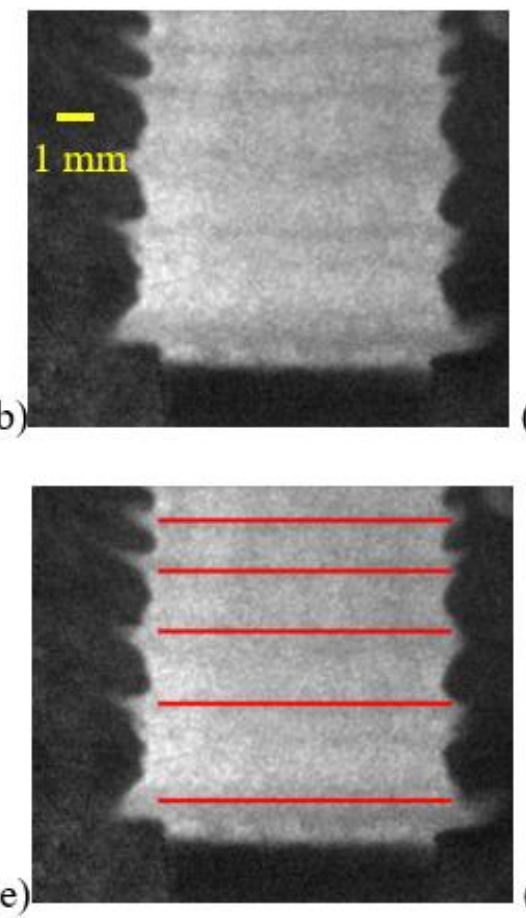

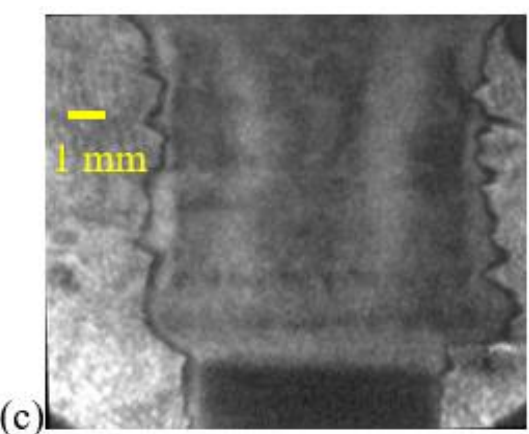

(f)

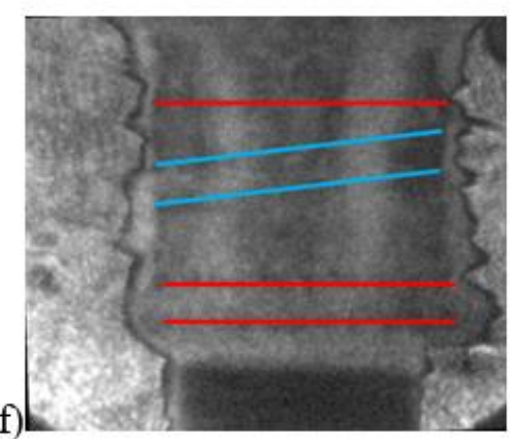

Figure 6.15: Contrast enhanced liner ablation images at $330 \mathrm{~ns}$ after start of current for (a) bare aluminum (b) plastic-on-inside aluminum and (c) plastic-on-outside aluminum. The same images are reproduced in (d) through (f) to highlight the presence of dark striations. These striations are exclusively horizontal and connect azimuthally correlated instability structures for cases (a) and (b); in case (c) both horizontal and angled striations are present. 
Figure 6.15 clearly shows that fully developed instability structures are highly

azimuthally correlated for the uncoated and plastic-on-inside cases, while the plastic-on-outside case shows significantly less correlation. The features highlighted in blue on Figure $6.15 f$

resemble the $\mathrm{m}=2$ instability, which has been previously observed on these liner explosions in the presence of an external magnetic field [43].

\subsubsection{Instability Growth Rate}

To quantify the differences between the three cases, the same instability fitting analysis described in Section 6.1 was applied to several shots of uncoated, plastic-on-inside, and plasticon-outside liner explosions. Fourier transforms taken at similar times are presented in Figure 6.16. 
Instability Fourier Transform, $\mathbf{t} \approx 220 \mathrm{~ns}$

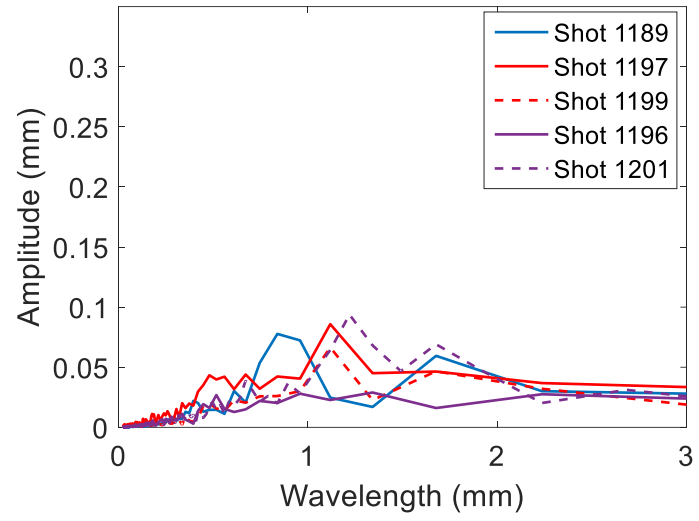

(a)

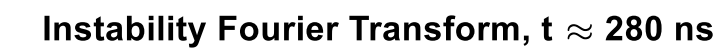

(c)

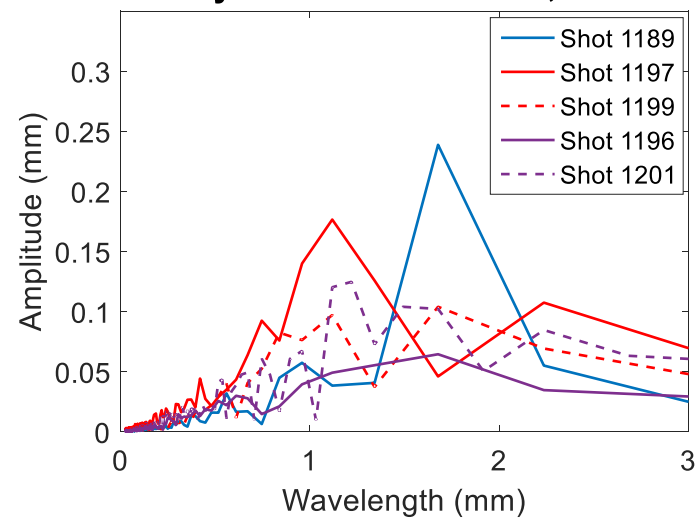

Instability Fourier Transform, $\mathbf{t} \approx 250 \mathrm{~ns}$

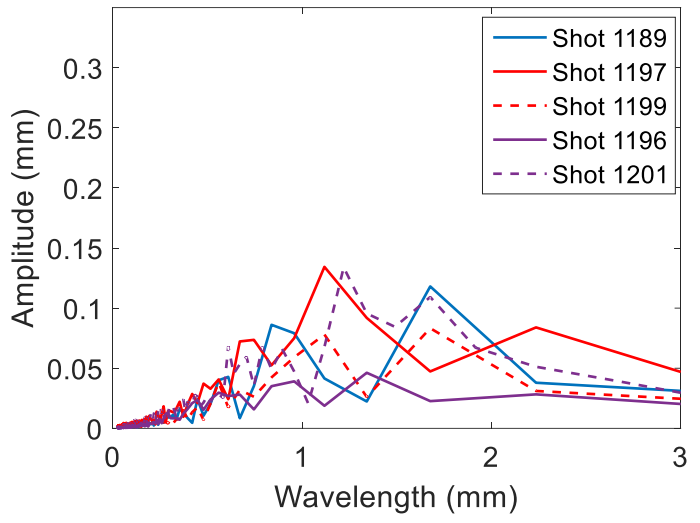

Instability Fourier Transform, $\mathbf{t} \approx \mathbf{3 0 0} \mathrm{ns}$

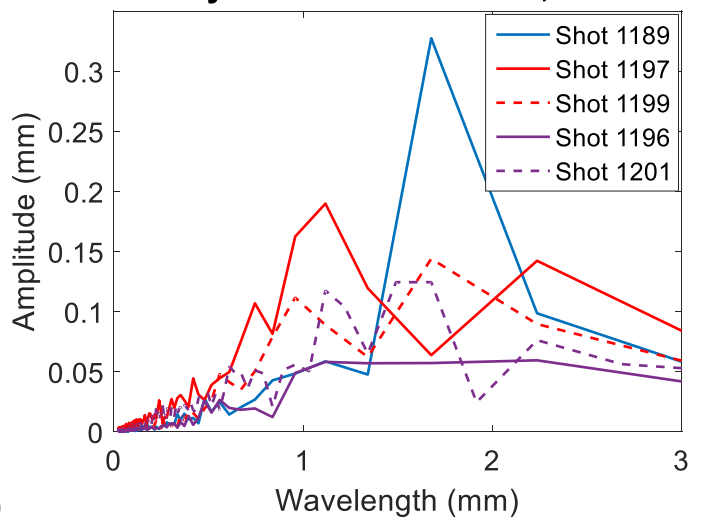

Figure 6.16: Fourier transforms of the interfaces for all 3 cases at various times after start of current. In general, instability structures grow more and become larger in wavelength for the bare aluminum shots (shown in blue) and plastic-on-inside shots (red) than for the plastic-on-outside case (purple).

From Figure 6.16, it is apparent that the uncoated foil exhibits both the most growth and the most dramatic transition of the instability to longer wavelengths. The plastic-on-inside shots have lower amplitude, but also exhibit a similar trend of longer (>1 $\mathrm{mm}$ ) wavelengths becoming dominant late in time. No discernable trend exists for the plastic-on-outside case. The average instability amplitude for the same shots is shown in Figures 6.17. 


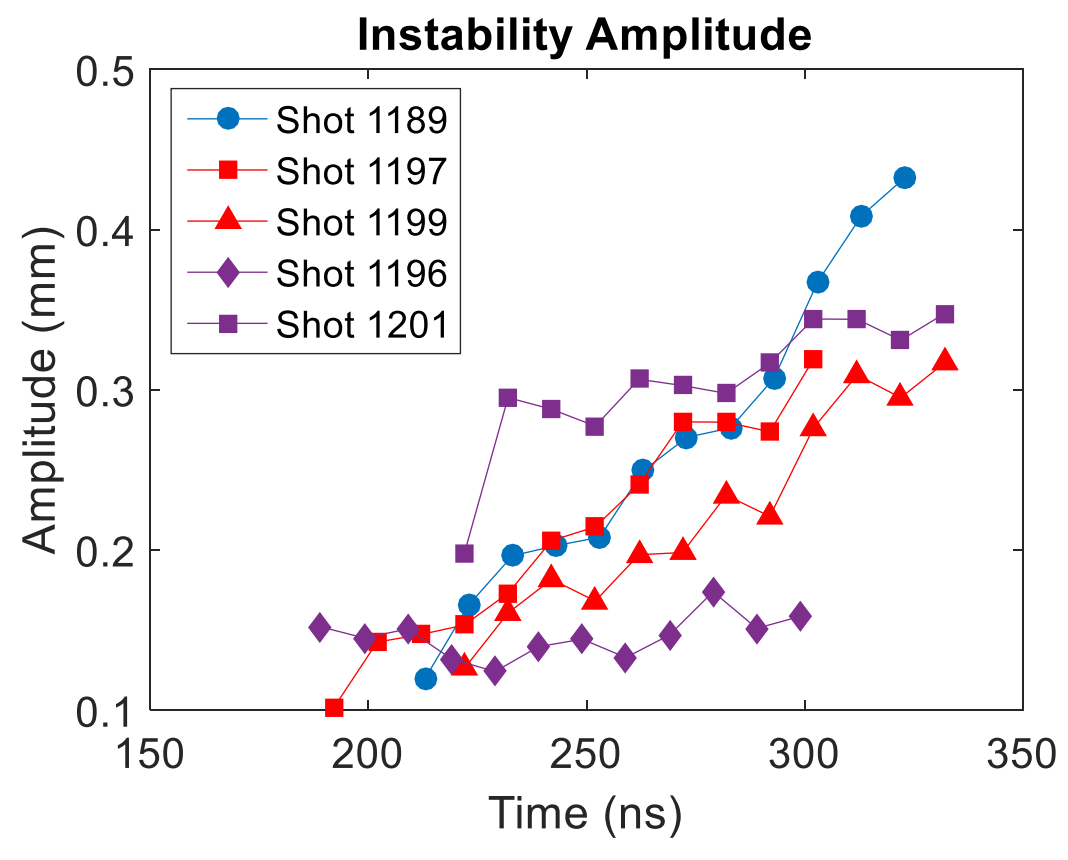

Figure 6.17: Instability amplitude as a function of time for all 3 cases. As expected, the bare aluminum shot (plotted in blue) exhibits the highest growth rate, and the plastic-on-inside cases (red) also show growth. While shot 1196 exhibits the expected behavior for the plastic-on-outside case (purple), shot 1201 shows a surprisingly large, albeit slowly growing, instability amplitude.

In Figure 6.17, the growth is similar for the plastic-on-inside and uncoated cases, The uncoated case shows the most late-time sausage mode growth because the overall plasma density is lower (since it does not have the additional mass from the plastic liner). Shot 1196, a plasticon-outside shot, shows almost no instability growth, consistent with previous observations that a dielectric coating damps the ETI and reduces the magnitude of seeded plasma instabilities [6, 11]. While the other plastic-on-outside shot, shot 1201, also shows a low growth rate, the initial amplitude is unusually large. The reason for this is not currently understood and merits further investigation.

\subsubsection{Azimuthal Correlation}

The differences in azimuthal symmetry observed on Figures 6.12 through 6.14 and highlighted on Figure 6.15 were quantified using the interface correlation function 


$$
C=\frac{\int_{L_{1}}^{L_{2}} \Delta x_{\text {right }} \Delta x_{\text {left }} d y}{\sqrt{\int_{L_{1}}^{L_{2}} \Delta x_{\text {right }}^{2} d y \int_{L_{1}}^{L_{2}} \Delta x_{\text {left }}^{2} d y}}
$$

where $L_{1}$ and $L_{2}$ are the bounds of the region of interest, $\Delta x_{\text {right }}$ and $\Delta x_{\text {left }}$ are the interface position functions, and $y$ is the axial dimension. The value $C$ is equal to 1 if the two interfaces are identical, - 1 if they are anticorrelated, and 0 if they show no correlation; intermediate values of $C$ quantify the degree of correlation. Figure 6.18 shows this correlation function for the same shots analyzed in Figures 6.16 and 6.17. On Figure 6.18, $C$ is shown as a function of time starting at $250 \mathrm{~ns}$, a time by which the instability structures have become large enough such that small errors in the image rotation do not significantly impact the calculated value.

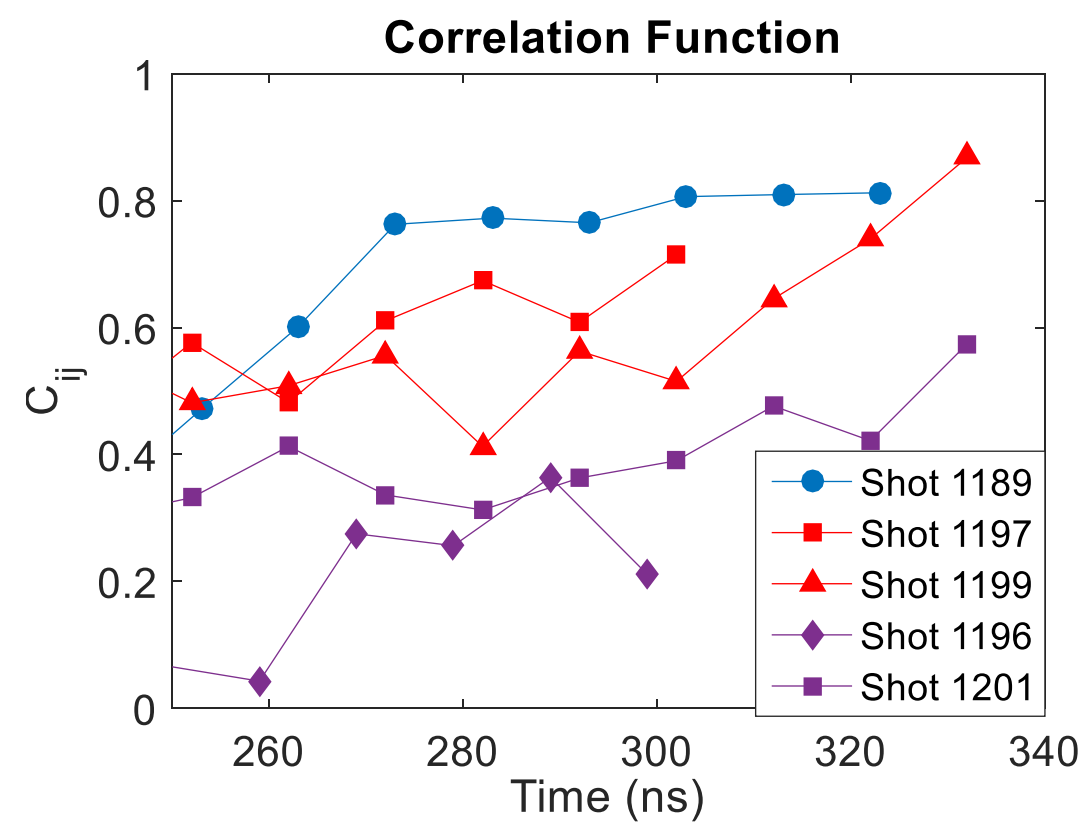

Figure 6.18: Interface correlation function vs time for all 3 cases calculated from (6.2). As anticipated, the bare aluminum (blue) and plastic-on-inside (red) cases are highly azimuthally correlated, while the plastic-on-outside (purple) shots exhibit markedly less correlation.

Figure 6.18 agrees with qualitative observations of the framing camera images: the uncoated and plastic-on-inside liners exhibit a higher degree of azimuthal correlation than the 
plastic-on-outside liners. These findings support the proposed explanation that ETI selfazimuthally correlates and seeds instabilities on exploding thin aluminum liners. While these liners are unstable to both $\mathrm{m}=0$ and higher order azimuthal modes with similar growth rates, in the absence of stronger seeding mechanisms ETI provides the azimuthally-correlated initial interface perturbation that grows into the experimentally observed sausage mode [43]. When a dielectric coating is included to suppress ETI, this azimuthal symmetry disappears, and hydrodynamic instability modes with $\mathrm{m}=0$ and $\mathrm{m}>0$ grow simultaneously. 


\section{CHAPTER 7}

\section{Conclusion and Suggestions for Future Work}

The MAIZE LTD has been used as a driver for various plasma instability studies, particularly for experiments relevant to magnetic liner implosion fusion. In this work, experiments were performed both on MAIZE and on a smaller pulsed power device to study the striation (perpendicular-to-current) form of the electrothermal instability, which has been hypothesized as a seed for plasma instabilities on liner implosions. The small pulsed power generator was used in conjunction with an optically magnified, ultrafast framing camera imaging system to study the growth of ETI as a temperature perturbation on initially solid, ablating aluminum foils. MAIZE was primarily employed to investigate the seeding of plasma instabilities, particularly MRT and the $\mathrm{m}=0$ sausage mode, from ETI. In addition to the ETI studies, the ability to predict the current and voltage output of MAIZE was greatly expanded, and a diagnostic method was developed to measure inductance changes due to load pinches requiring only a current measurement.

Studies on the MAIZE LTD are highly relevant to MagLIF because the proposed next generation of MagLIF experiments will be conducted on a large current drive facility consisting of many LTDs [7-10]. A computational model for analyzing current and voltage delivered to the load was developed. It was demonstrated that peak current could vary by 100 s of kA and risetime could vary by over $100 \mathrm{~ns}$ for a realistic range of load resistance and inductance parameters. The effects of switch trigger time delay introduced by aging switches were observed 
and quantified, and a variety of performance issues including abnormally high risetimes, abnormally low peak currents, and shorts in the transmission line (which is assumed to be magnetically insulated from arcs) were traced to the switches. Changes in inductance due to load pinching (a desirable phenomenon for a variety of x-ray production experiments, instability studies, and the MagLIF project itself) were shown to impact the current delivered by the driver during the course of a single shot. These current changes were measured for pinches of wire arrays and metallic liners and used in conjunction with the circuit model and initial conditions on charging voltage to measure load inductance as a function of time. The current model was adapted to provide these measurements even in the presence of large, random switch firing delay. Inductance measurements yield insight on pinch timing and strength, and can improve measurements of effective current-carrying pinch-column radius compared to laser shadowgraphy alone. Previous such methods [53-55] required an additional measurement of voltage, which is more difficult to obtain than a measurement of current alone.

The ETI experiments, which comprise the bulk of this work, were performed in two experimental campaigns - one on MAIZE and one on a smaller, single-capacitor pulsed power device. The small pulse generator was chosen because its low peak current (4 kA) and long risetime (600 ns) prolong the transition from the solid phase to the vapor phase for metallic loads, which has been shown to correlate with more ETI e-folds on previous simulations [19, 23]. This device was used to ablate thin ( $400 \mathrm{~nm}$ to $2 \mu \mathrm{m}$ thickness) planar aluminum foilsvarious gas and pressure conditions. A 12-frame, gated ICCD with maximum frame rate of $2 \times 10^{8} \mathrm{~s}^{-1}$ and minimum exposure time of $5 \mathrm{~ns}$ was used in conjunction with an optical magnification lens system to obtain time-resolved self-emission images of the ablating foils with up to $2 \mu \mathrm{m}$ spatial resolution. Electrical and spectroscopic measurements established that areas 
on the surface of the foil emitting visible light were due to the presence of low-electron temperature, ionized aluminum plasma resulting from localized resistive heating. The framing camera was calibrated to correlate camera response with emitting temperature assuming blackbody emission, which was shown to be dominant over line emission and other continuum emission processes. This process allowed the framing camera to be used as a temporally and spatially resolved surface temperature diagnostic.

Temperature perturbations on the foil surface were analyzed as a function of time to compare with ETI theory. Minimum growing perturbation size was on the order of $10 \mathrm{~s}$ of $\mu \mathrm{m}$, consistent with previous theoretical [19] and experimental [20] observations on wire ablations, as well as with minimum growing wavelength calculated using linear ETI theory for the current densities observed on these foil ablations. Obtaining 12 frames per shot provided the first ever time-resolved measurements of ETI growth as a temperature perturbation on ablating material. Instability growth rates were calculated from several shots by taking lineouts along the direction of current, fitting each lineout to an exponential growth function, and averaging the fitted growth rates over many lineouts. The resulting measured growth rates showed remarkable agreement with linear ETI theory, with most shots falling within $25 \%$ of the window set by the maximum and minimum growth rates for observed feature sizes, despite occurring in a nonlinear regime where $\delta T / T$ is greater than unity. Additionally, growth rates exhibited the theoretically predicted $J^{2}$ dependence on current density for similar conditions on the unperturbed foil material.

Experiments on MAIZE were conducted to study the coupling of ETI to the MRT and sausage instabilities. Liners of aluminum and titanium with plastic coatings on the inner surface were imploded using a dumbbell geometry [37] to study MRT growth. Titanium was originally 
believed to be more unstable to ETI due to its higher resistivity, and derivative of resistivity with respect to temperature, than aluminum. However, little difference in instability formation was observed between the two cases. It was noted that aluminum and titanium have similar values of the ratio $T_{\text {crit }} / T_{\text {melt }}$, where $T_{\text {crit }}$ is the critical temperature and $T_{\text {melt }}$ is the melting temperature. According to ETI theory presented by Oreskhin [19], this ratio is responsible for determining the integral amount of ETI growth; materials with similar integral growth rates provide similar interface perturbations for MHD instability growth. To evaluate this claim, liners of aluminum and tantalum were exploded on solid plastic supports, a geometry previously shown to grow pronounced, easily identifiable sausage instabilities on MAIZE [43]. Tantalum has one of the lowest ratios of $T_{\text {crit }} / T_{\text {melt }}$ of any practically available foil material. The plasma-vacuum interface on exploding tantalum liners showed little to no instability structure over 100s of nanoseconds; during equivalent time the sausage instability grew to an amplitude that was on the same order as the plasma column radius for comparable aluminum liners. This provides substantial evidence that ETI in fact seeds hydrodynamic instabilities on ablating metal loads.

The previous exploding liner experiments on MAIZE [43] observed preferential seeding of the $\mathrm{m}=0$ sausage mode when no external seeding mechanisms were applied, despite the comparable (and slightly larger) growth rates of competing $\mathrm{m}>0$ modes. Because ETI tends to self-azimuthally correlate, it was proposed as the seeding mechanism responsible for the observed growth of the azimuthally correlated $m=0$ mode. To test this hypothesis, liners of aluminum were exploded on solid plastic supports with no externally applied seeding for three cases: bare aluminum, aluminum with plastic coating on the inside surface, and aluminum with plastic coating on the outside surface. The plastic-on-outside geometry has been previously shown $[6,11]$ to damp ETI and subsequent instability growth. These experiments showed that 
the plastic-on-outside geometry significantly disrupted the azimuthal symmetry observed on the uncoated and plastic-on-inside shots. As a whole, the experiments in this dissertation provided experimental evidence for the growth of ETI on initially solid, metallic loads; demonstrated instability growth rates in good agreement with theory; and linked conditions designed to limit ETI growth through material selection and dielectric coating with reduced hydrodynamic instability amplitude and azimuthal symmetry.

Potential avenues for future work include further investigation into the plastic coating shots, particularly to investigate the apparent outlier shot that showed a large instability amplitude but little growth compared to the uncoated shots at similar times. It would also be interesting to conduct liner explosions with low frame-rate imaging, such as those shown in Figures 6.8 through 6.10 for additional materials with values of $T_{\text {crit }} / T_{\text {melt }}$ that lie between those of tantalum and aluminum. Measurements of initial foil thickness, for example using scanning electron microscopy (SEM), may also shed additional insight on the foil ablation dynamics. While thickness perturbations are not expected to impact the growth rates of ETI measured in this work, since growth rate is independent of initial seeding mechanism, quantifying thickness perturbations may elucidate the reasons behind the difference in feature sizes observed on the $2.0 \mu \mathrm{m}$ foils compared to those on the $400 \mathrm{~nm}$ and $800 \mathrm{~nm}$ foils.

Finally, additional studies could focus on the growth of parallel filamentations that arise late in time on several foil ablation shots. One of these shots is reproduced below in Figure 7.1 for reference. These filamentations are believed to be due to the $\frac{\partial \eta}{\partial T}<0$ form of ETI that arises in the plasma phase, once expanding plasma plumes from initially ablating striations have merged. In order to analyze the growth of these filaments, a suitable model of plasma resistivity would have to be employed. Spitzer resistivity is a poor approximation for the plasmas 
generated on these atmospheric foil ablations, as the ionization fractions are believed to be very low $(<1 \%)$. Additionally, the neutral density of this partially ionized foil vapor is large, such that the collision frequency is expected to be large compared to the plasma frequency. If a proper model for plasma resistivity is employed that takes these points into account, the transition from striation ETI to filamentation ETI could be investigated and compared with theory for shots such as the one shown in Figure 7.1.
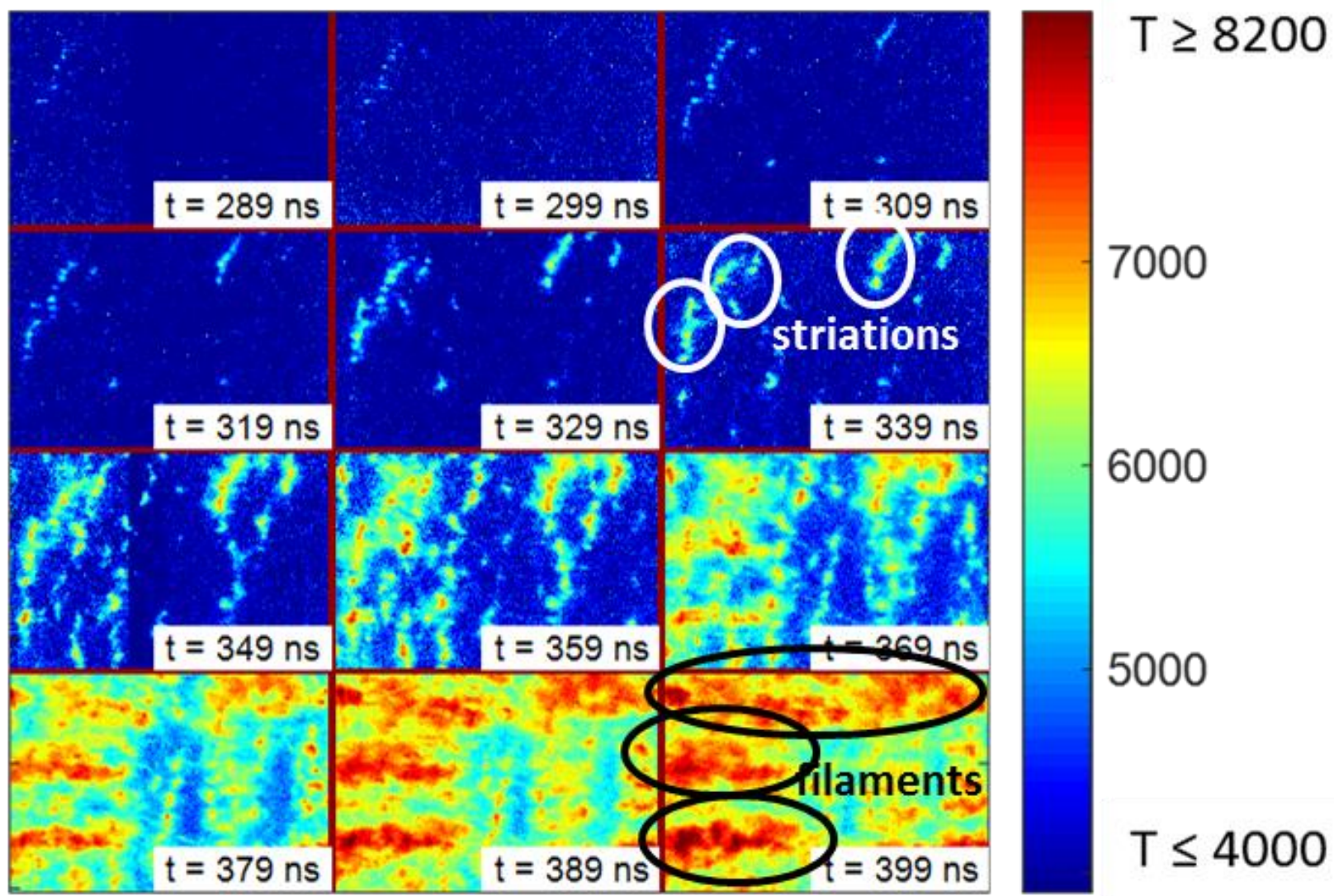

Figure 7.1: Temperature surface plot of an $800 \mathrm{~nm}$ x $0.7 \mathrm{~cm}$ x $0.9 \mathrm{~cm}$ aluminum foil ablation showing plasma from vaporized sections of the foil joining to form filamentary structures late in time. 


\section{APPENDIX A}

\section{LTSPICE Simulation of MAIZE with Independent Brick Trigger Timings}

The following code was used to generate a model of the MAIZE LTD in LTSPICE. This model allows independent brick trigger timings, which allows the user to represent switches firing at different times.

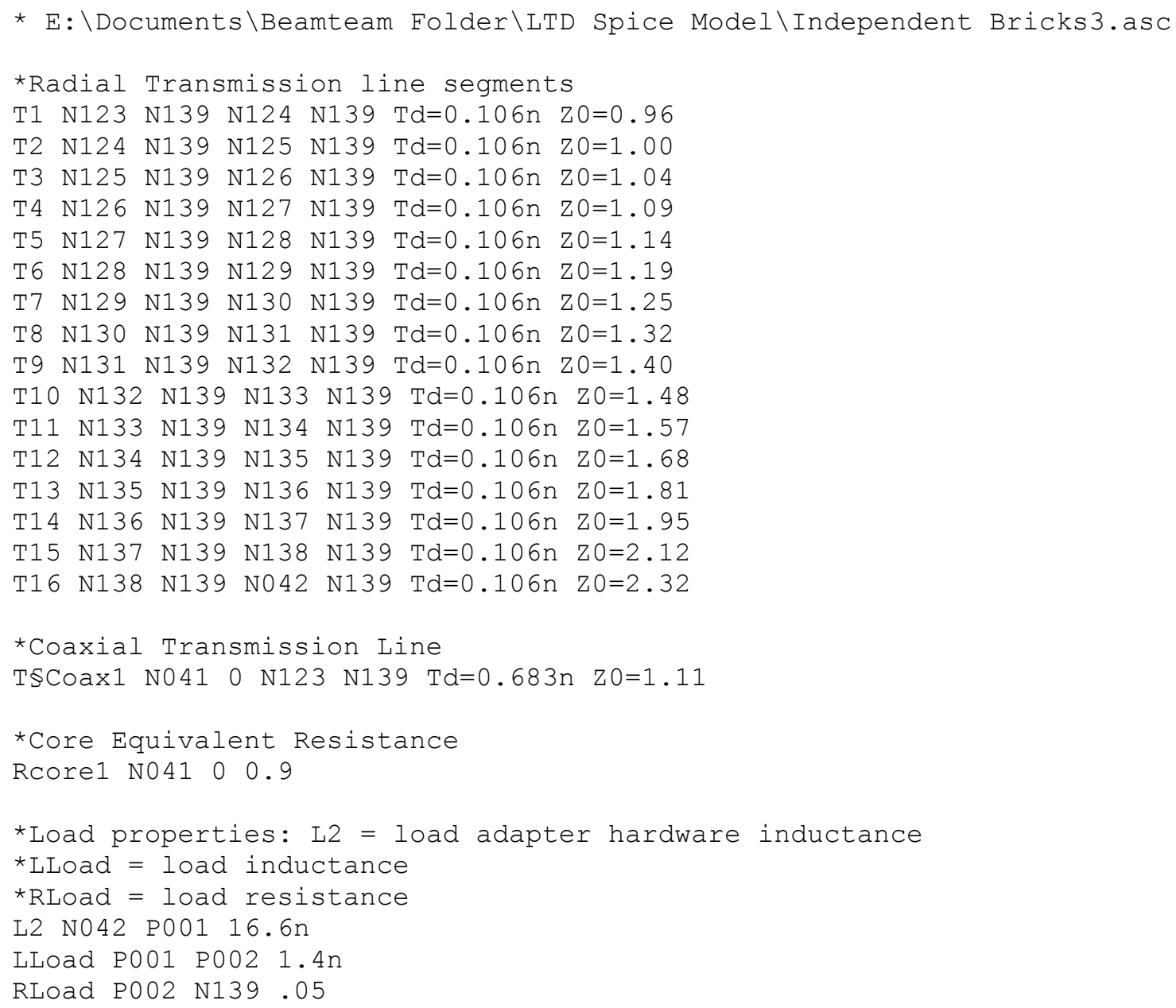




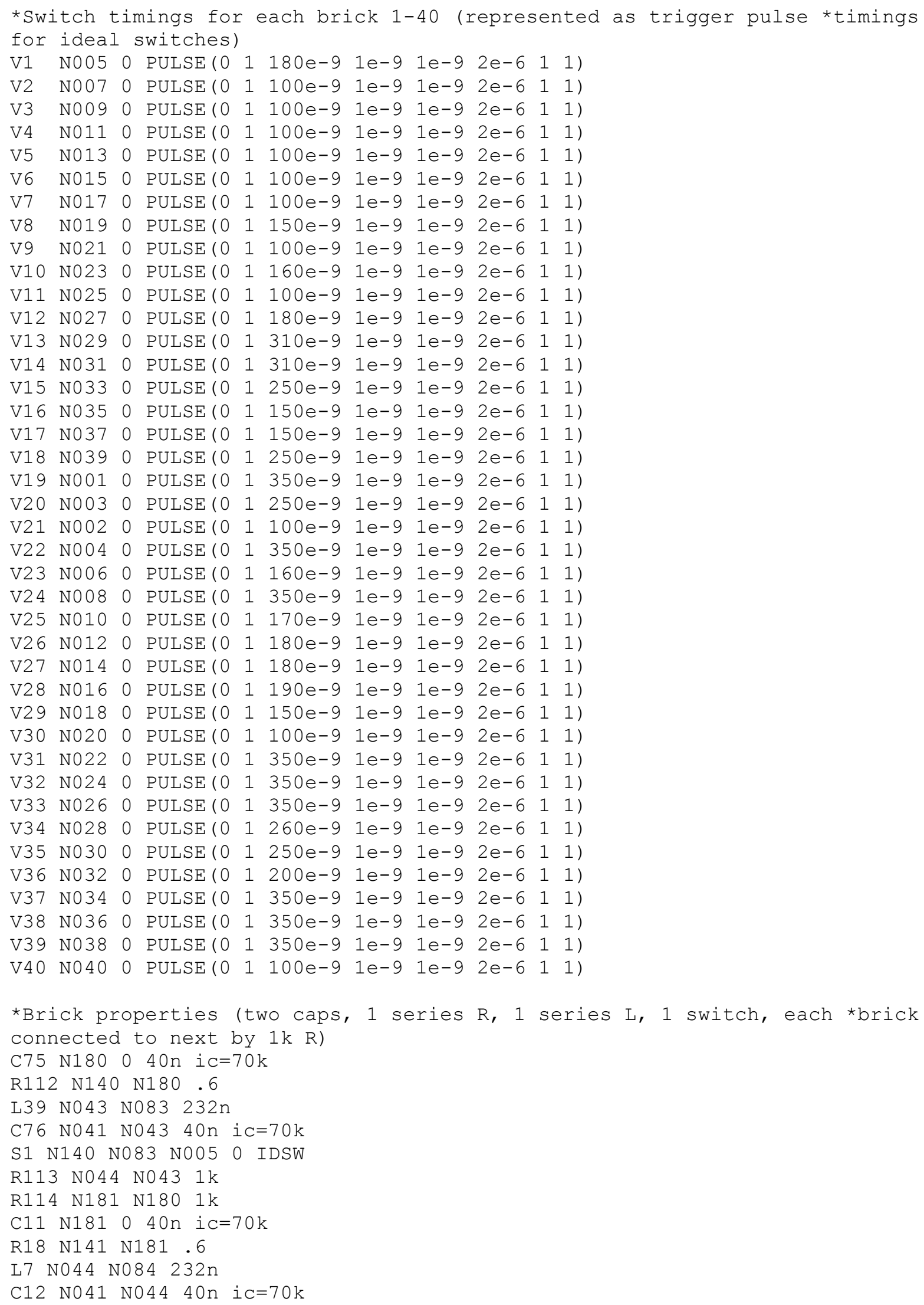




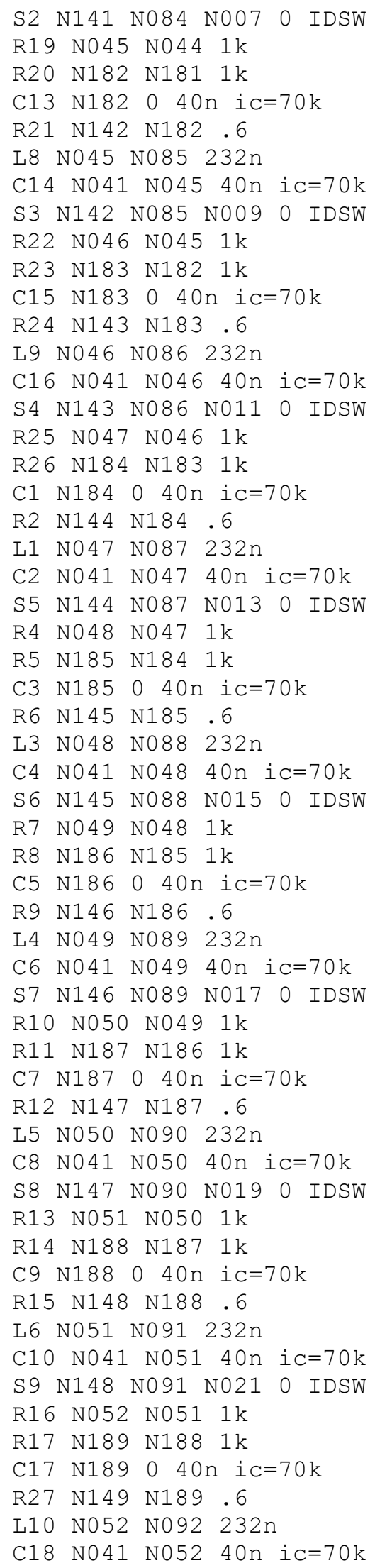




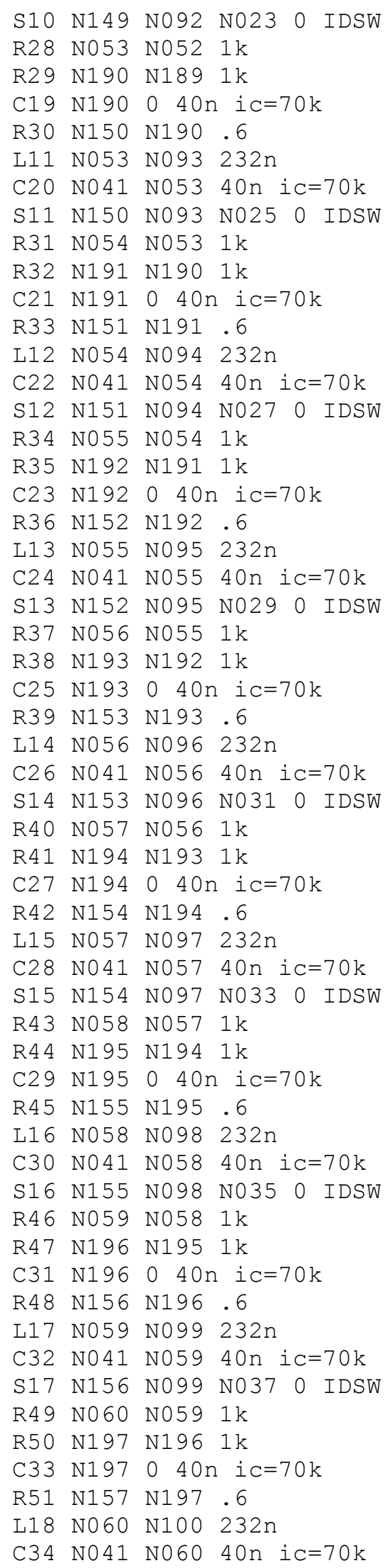




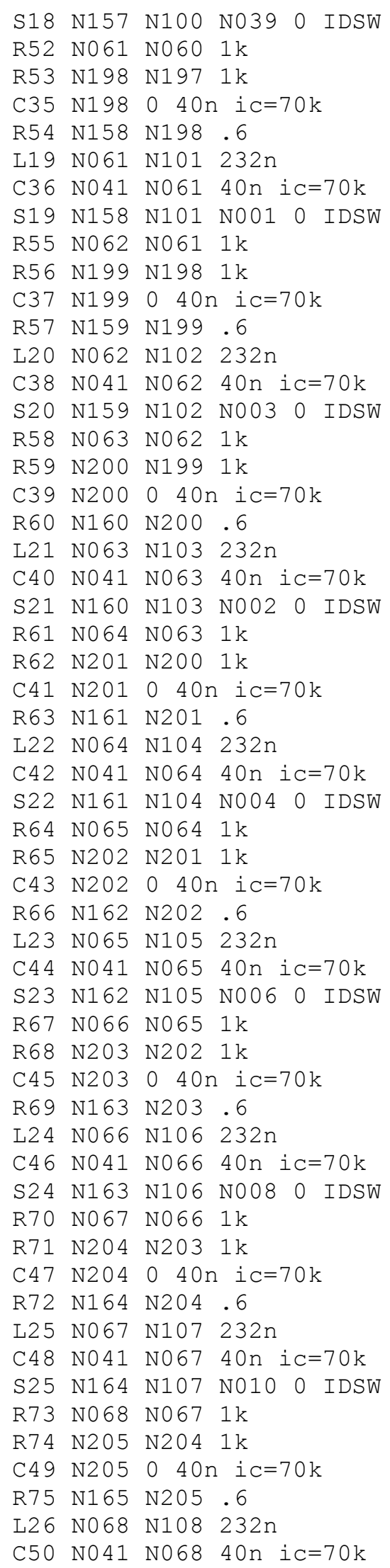




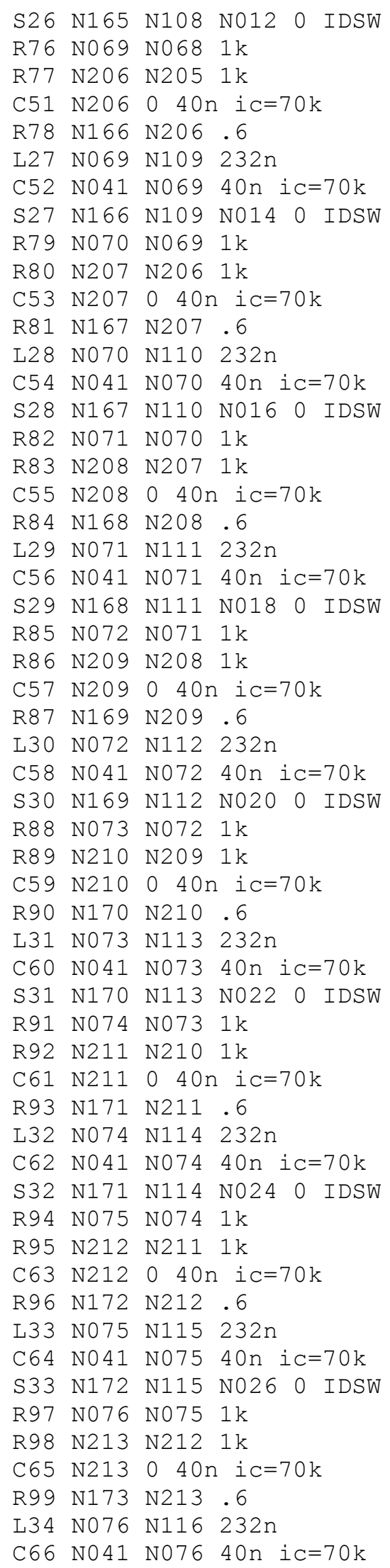




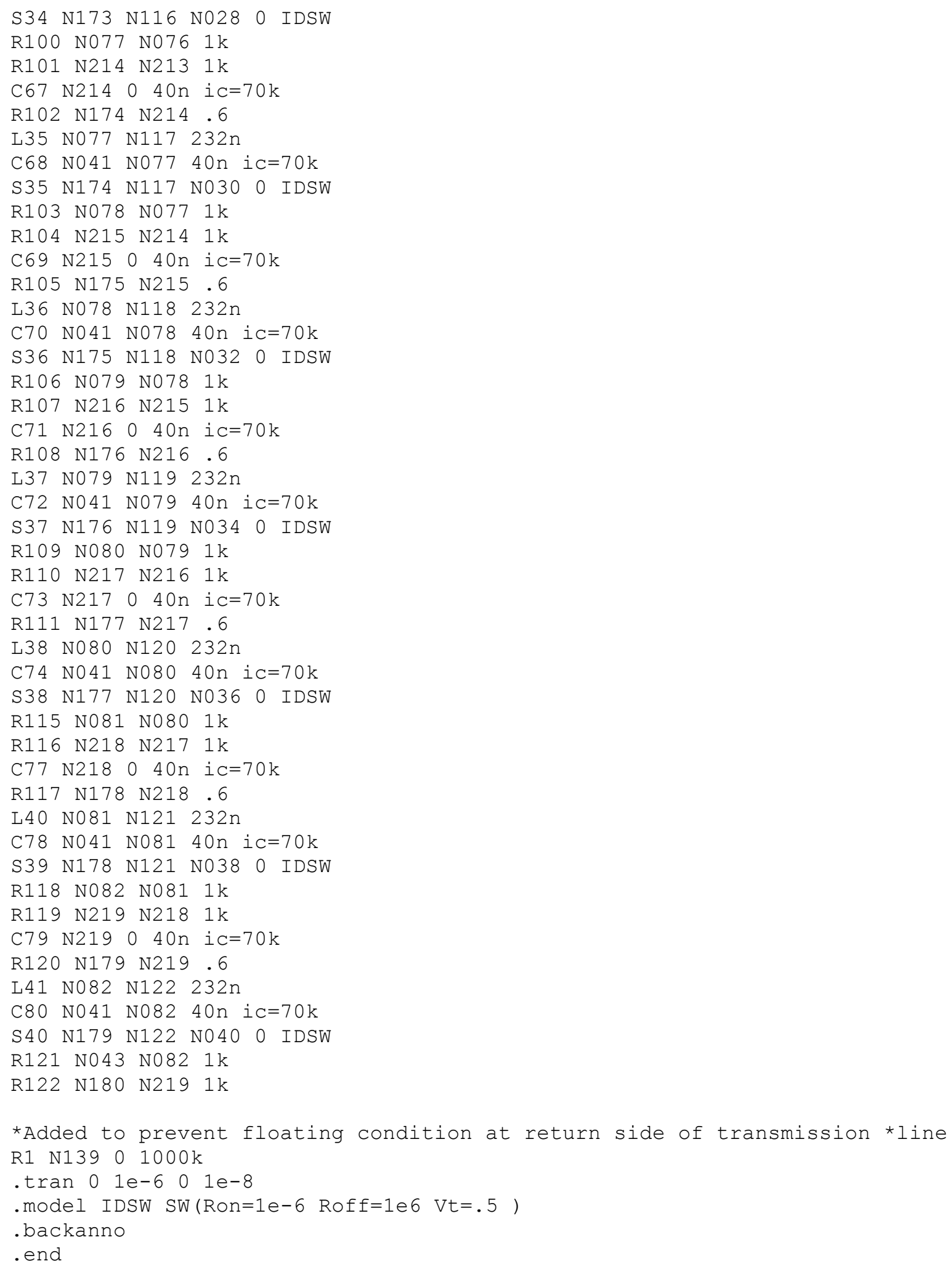




\section{APPENDIX B}

\section{MATLAB Code for Simulating MAIZE and Calculating Load Inductance}

The following codes are MATLAB scripts designed to simulate the MAIZE LTD. Code

1 calculates voltage and current for specified time-averaged load parameters, code 2 performs a

parameter sweep over load inductance and resistance, and code 3 calculates time-dependent load

inductance from an input current trace, assuming constant load resistance.

CODE 1:

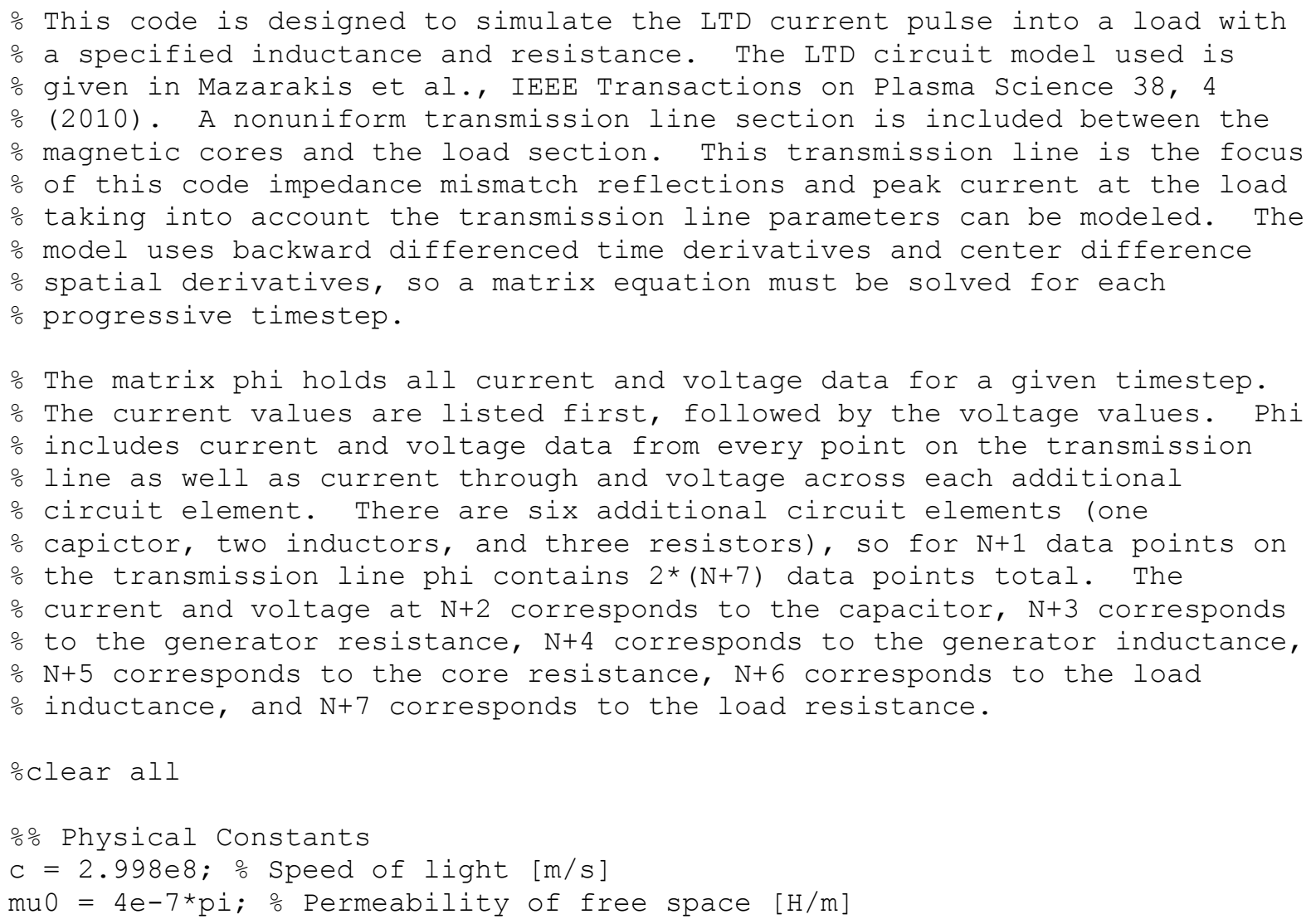




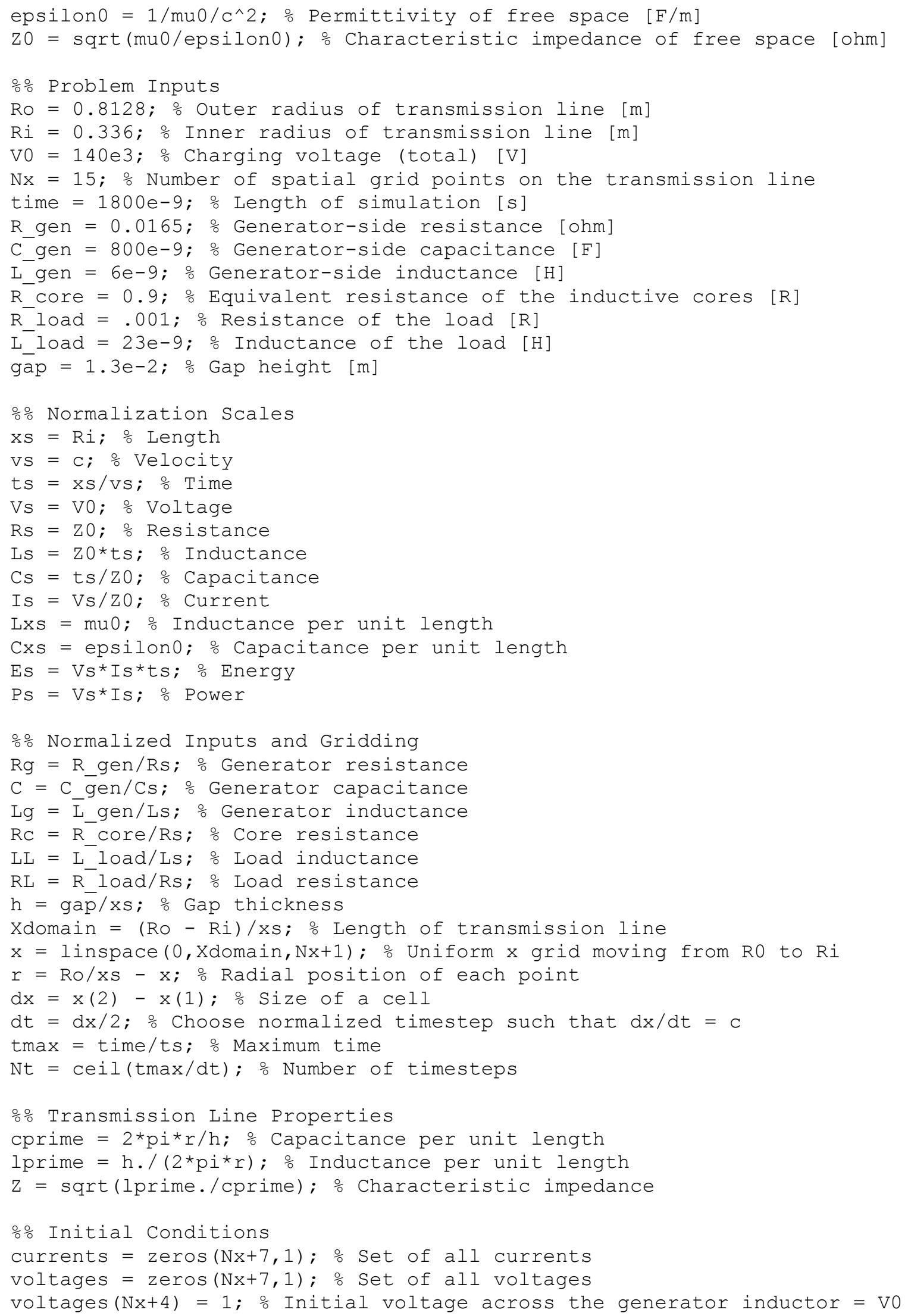




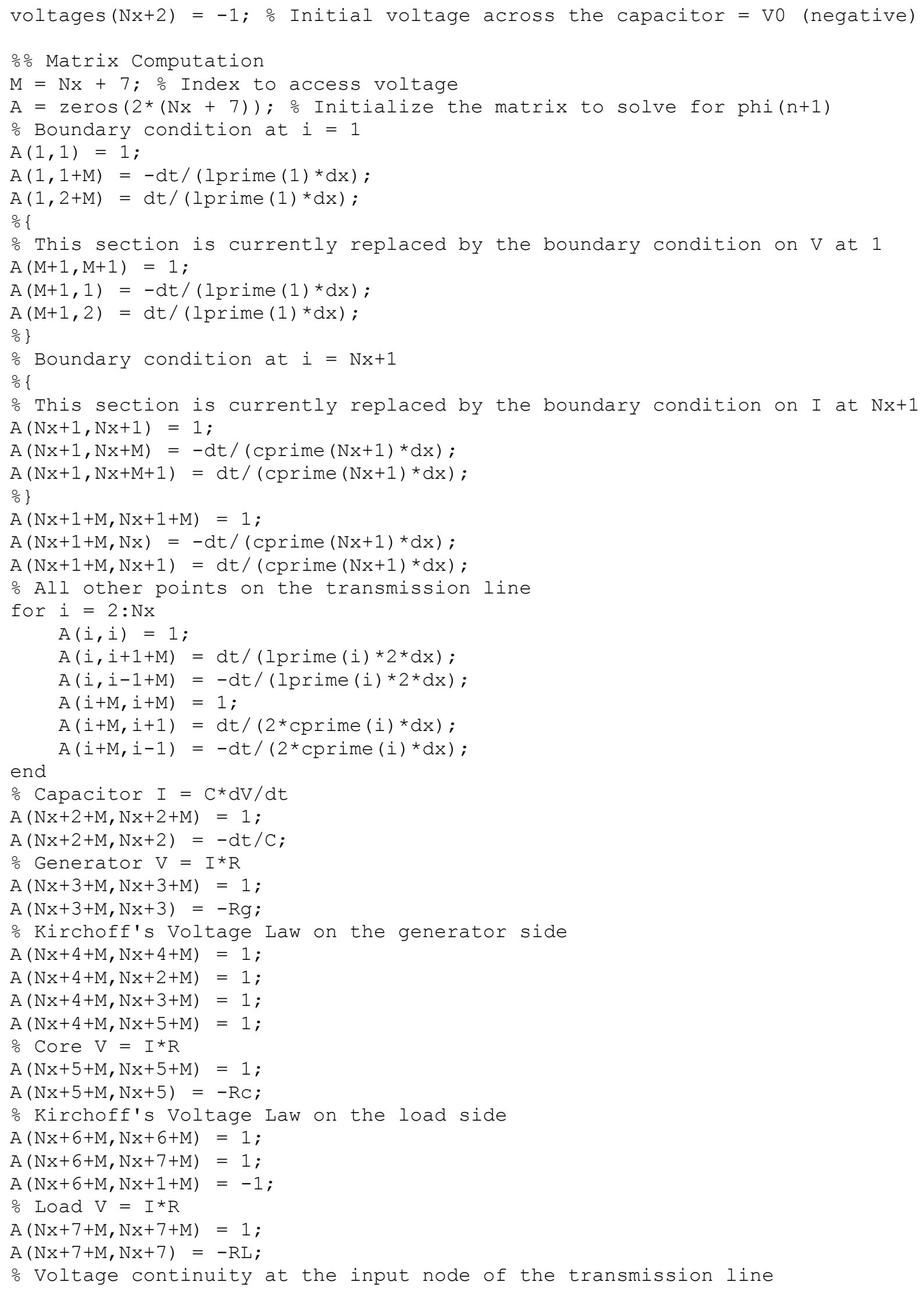




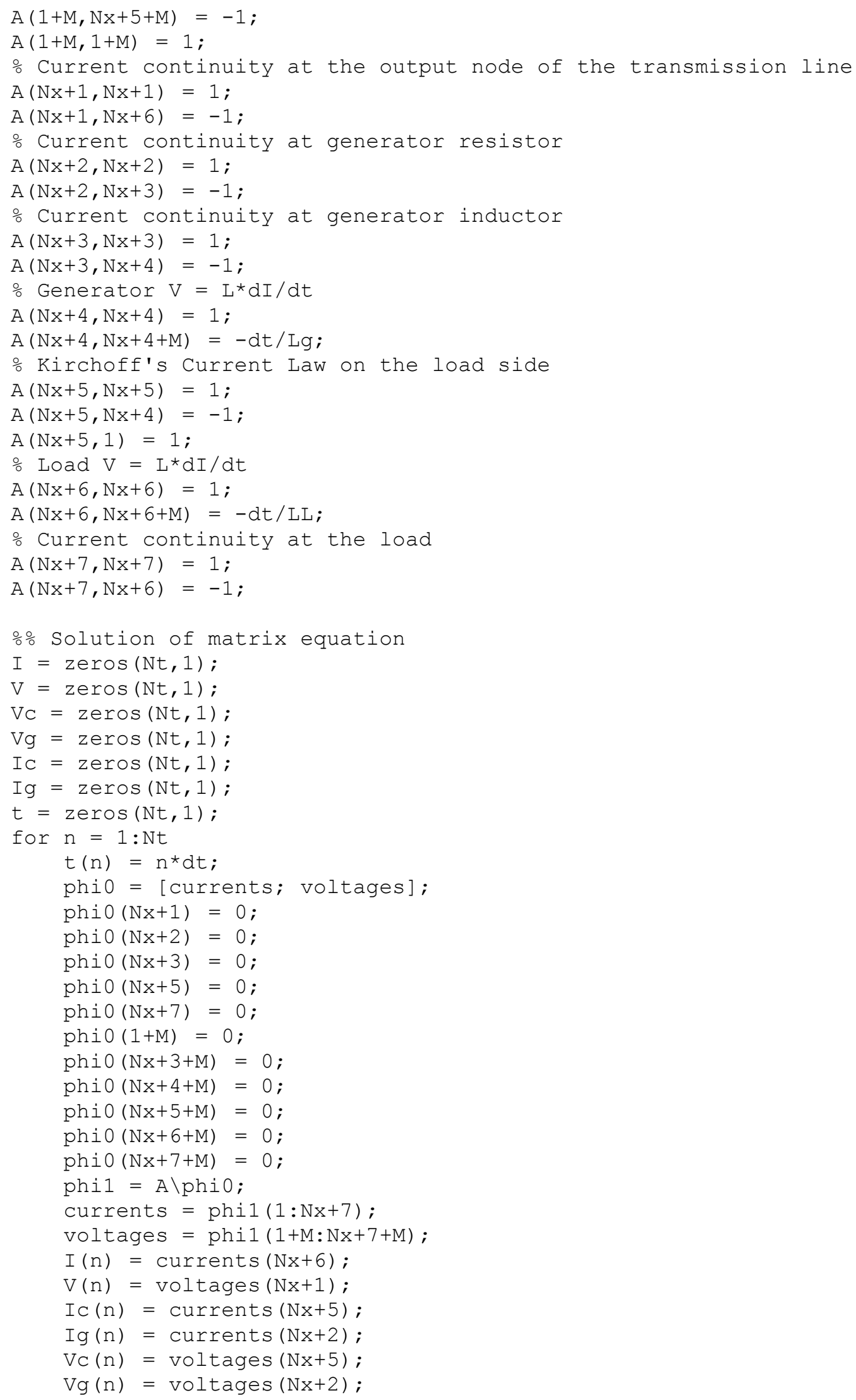


end 


\section{CODE 2:}

\% This code is designed to simulate the LTD current pulse into a load with

\% a specified inductance and resistance. The LTD circuit model used is

\% given in Mazarakis et al., IEEE Transactions on Plasma Science 38, 4

\% (2010). A nonuniform transmission line section is included between the

\% magnetic cores and the load section. This transmission line is the focus

\% of this code impedance mismatch reflections and peak current at the load

\% taking into account the transmission line parameters can be modeled. The

\% model uses backward differenced time derivatives and center difference

\% spatial derivatives, so a matrix equation must be solved for each

o progressive timestep.

\% The matrix phi holds all current and voltage data for a given timestep.

\% The current values are listed first, followed by the voltage values. Phi

o includes current and voltage data from every point on the transmission

o line as well as current through and voltage across each additional

o circuit element. There are six additional circuit elements (one

o capictor, two inductors, and three resistors), so for $\mathrm{N}+1$ data points on

\% the transmission line phi contains $2 *(N+7)$ data points total. The

\% current and voltage at $\mathrm{N}+2$ corresponds to the capacitor, $\mathrm{N}+3$ corresponds

\% to the generator resistance, $N+4$ corresponds to the generator inductance,

$\div \mathrm{N}+5$ corresponds to the core resistance, $\mathrm{N}+6$ corresponds to the load

o inductance, and $\mathrm{N}+7$ corresponds to the load resistance.

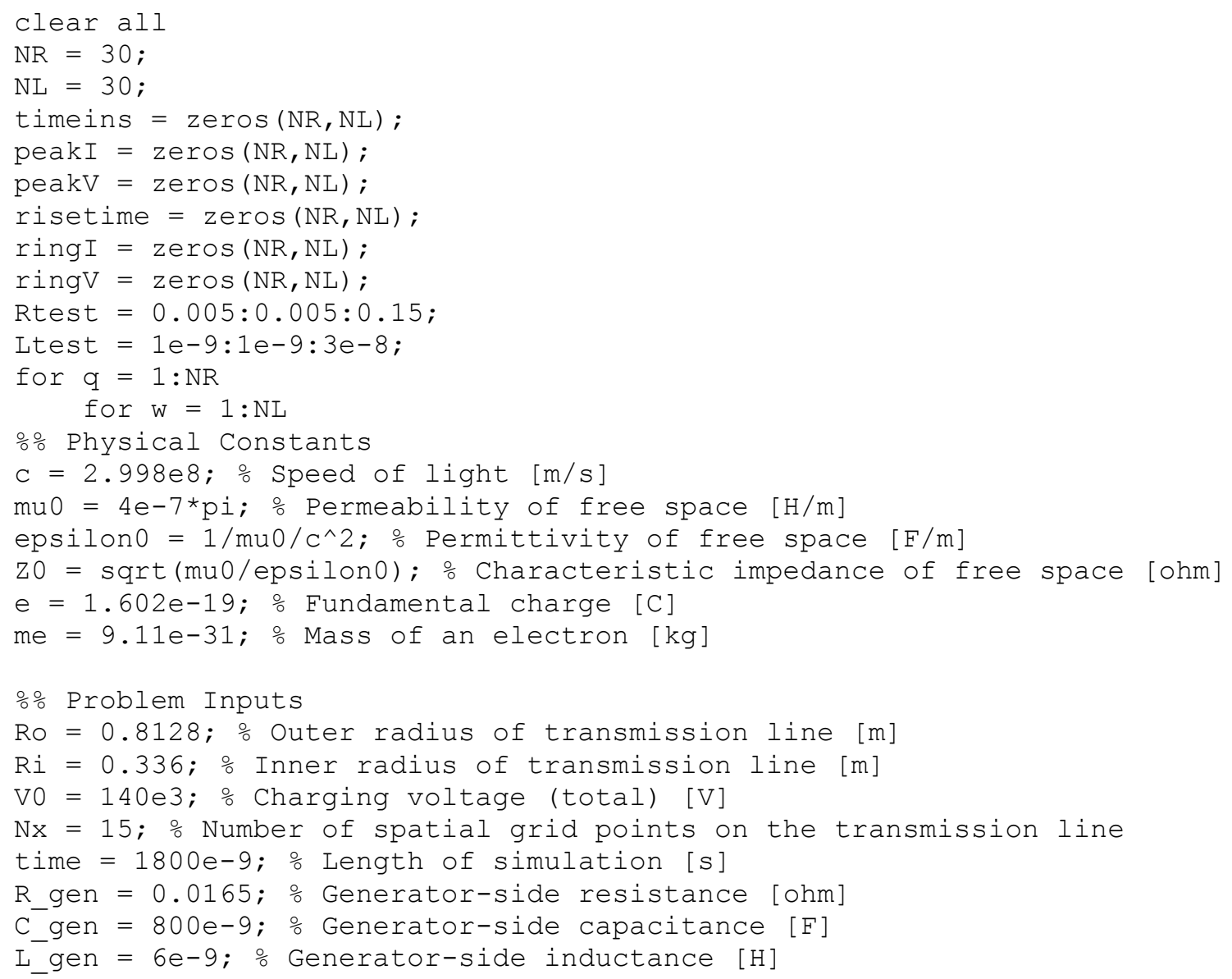




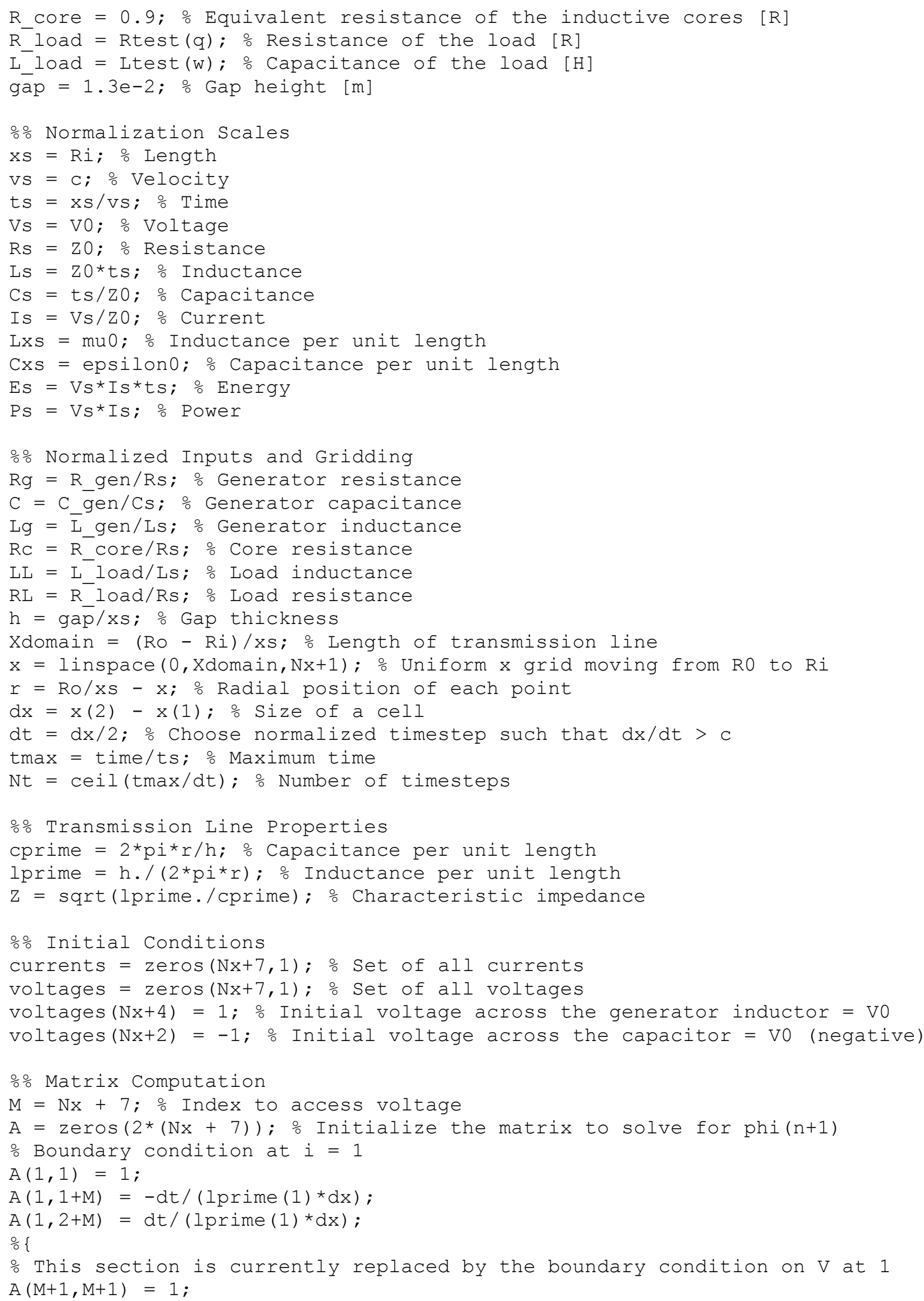




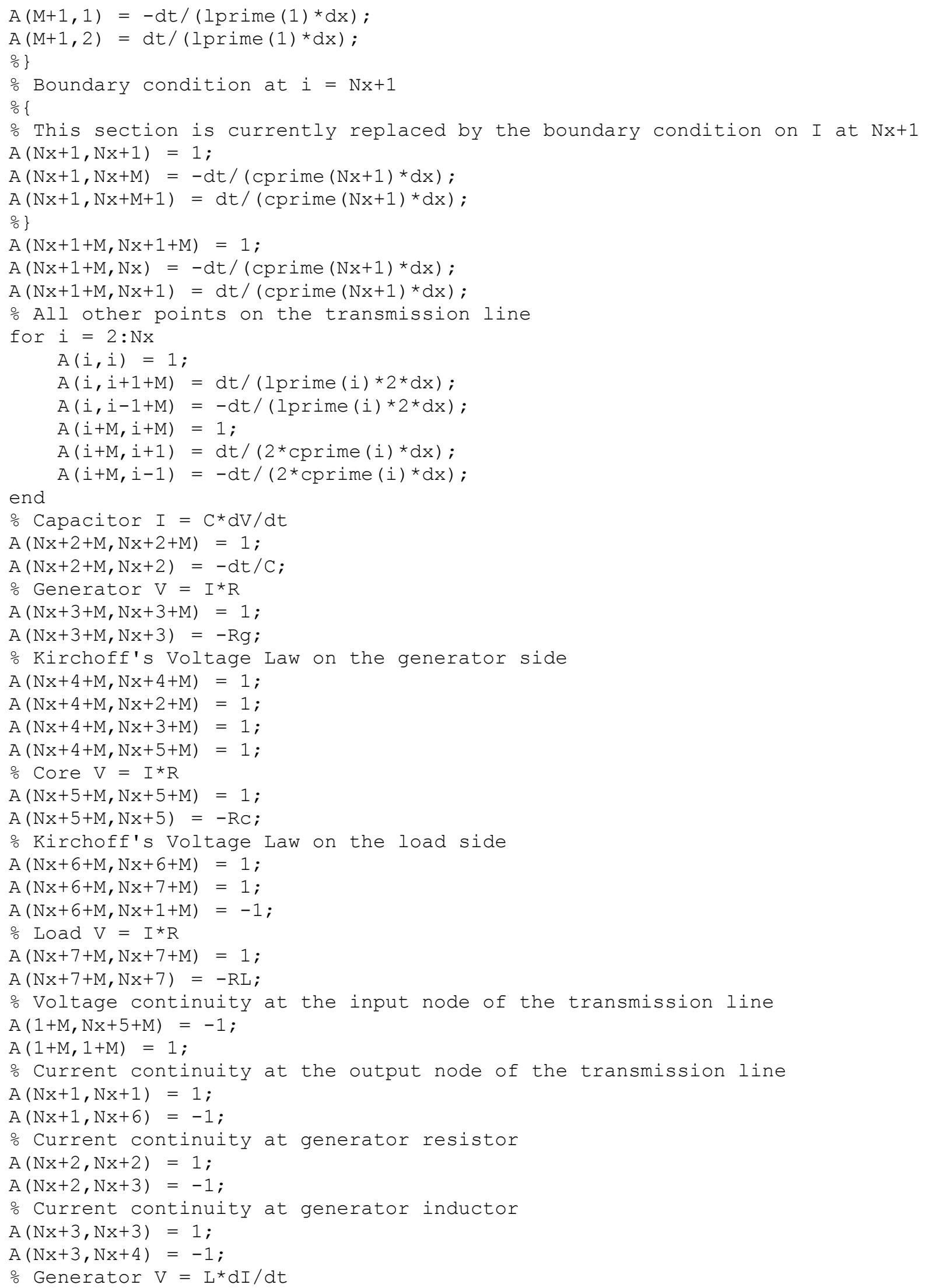




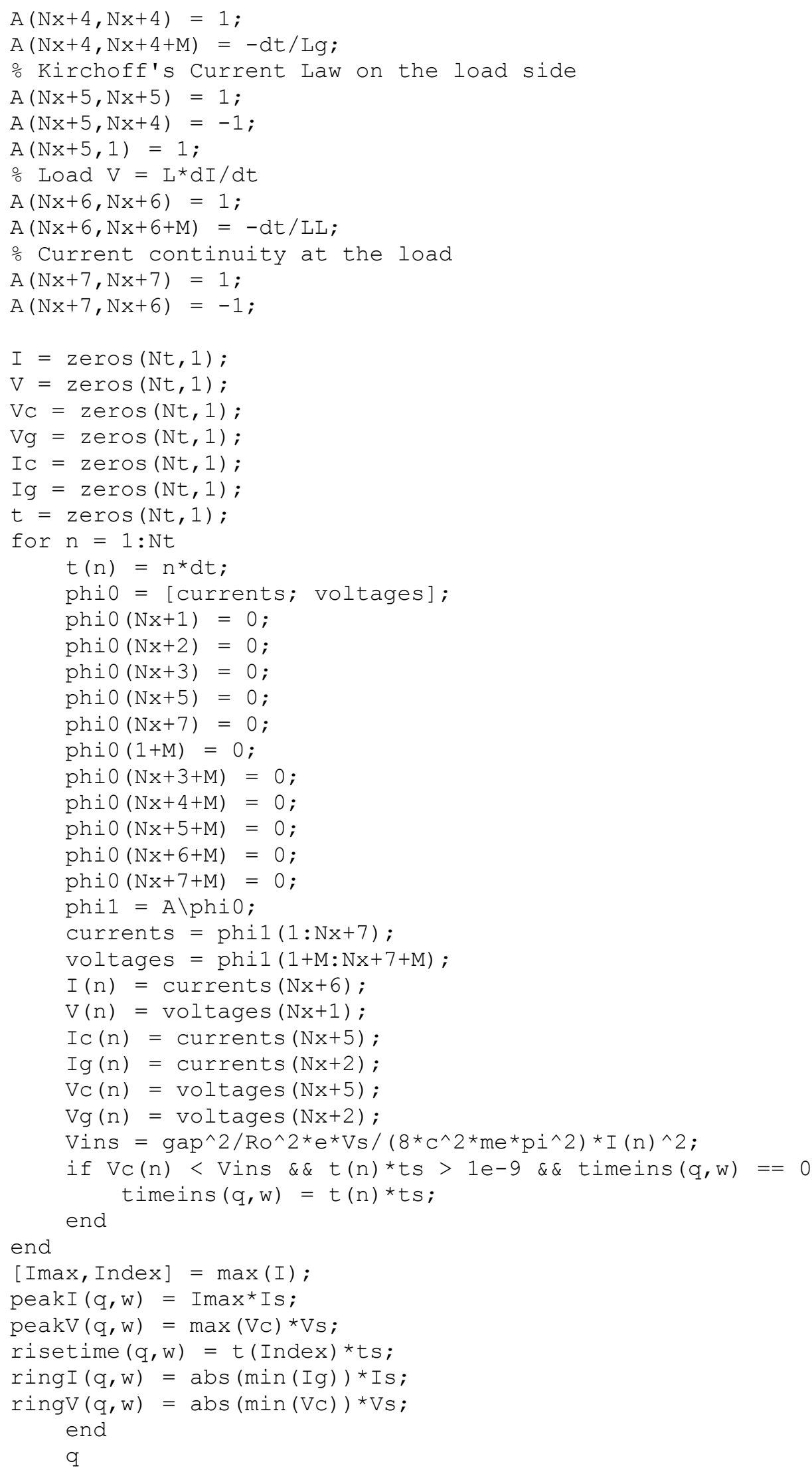




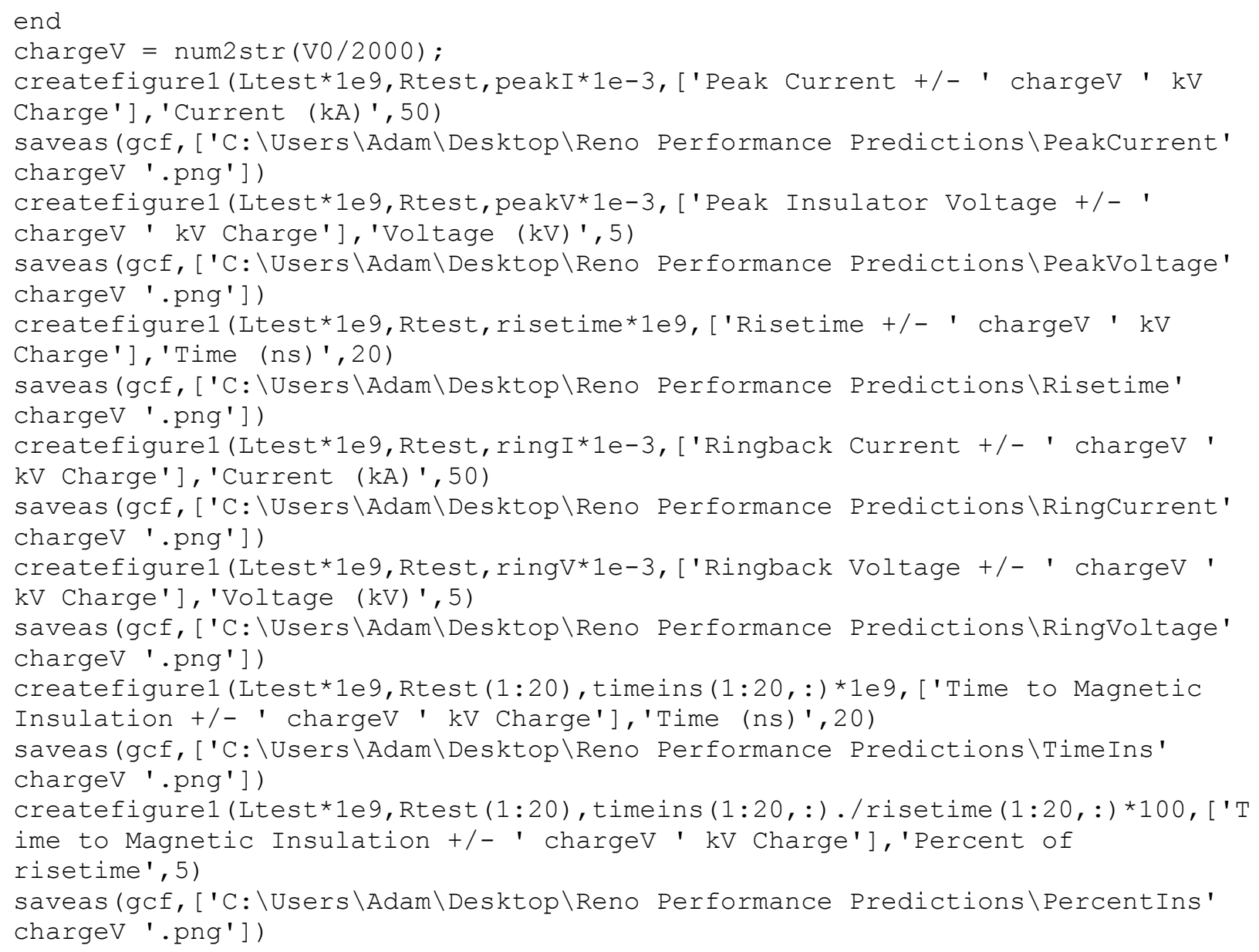




\section{CODE 3:}

\% This code is designed to simulate the LTD current pulse into a load with

\% a specified inductance and resistance. The LTD circuit model used is

\% given in Mazarakis et al., IEEE Transactions on Plasma Science 38, 4

\% (2010). A nonuniform transmission line section is included between the

\% magnetic cores and the load section. This transmission line is the focus

\% of this code impedance mismatch reflections and peak current at the load

o taking into account the transmission line parameters can be modeled. The

\% model uses backward differenced time derivatives and center difference

\% spatial derivatives, so a matrix equation must be solved for each

o progressive timestep.

\% The matrix phi holds all current and voltage data for a given timestep.

\% The current values are listed first, followed by the voltage values. Phi

o includes current and voltage data from every point on the transmission

o line as well as current through and voltage across each additional

o circuit element. There are six additional circuit elements (one

o capictor, two inductors, and three resistors), so for $\mathrm{N}+1$ data points on

\% the transmission line phi contains $2 *(N+7)$ data points total. The

\% current and voltage at $\mathrm{N}+2$ corresponds to the capacitor, $\mathrm{N}+3$ corresponds

\% to the generator resistance, $N+4$ corresponds to the generator inductance,

$\div \mathrm{N}+5$ corresponds to the core resistance, $\mathrm{N}+6$ corresponds to the load

o inductance, and $\mathrm{N}+7$ corresponds to the load resistance.

oclear all

응 Physical Constants

$\mathrm{c}=2.998 \mathrm{e} 8$; $\%$ Speed of light $[\mathrm{m} / \mathrm{s}]$

mu0 $=4 e-7{ }^{*}$ pi; $\frac{\circ}{0}$ Permeability of free space $[\mathrm{H} / \mathrm{m}]$

epsilono = $1 / \mathrm{mu} 0 / \mathrm{C}^{\wedge} 2$; $\frac{\circ}{0}$ Permittivity of free space $[\mathrm{F} / \mathrm{m}]$

$\mathrm{ZO}=\operatorname{sqrt}(\mathrm{mu} 0 / \mathrm{epsilon0)} ;$ o Characteristic impedance of free space [ohm]

응 Problem Inputs

pfire $=1$; $\%$ Percent of machine firing

Ro $=0.8128 ;$ o Outer radius of transmission line [m]

$\mathrm{Ri}=0.69 / 2$; $\frac{\circ}{0}$ Inner radius of transmission line [m]

$\mathrm{VO}=140 \mathrm{e} ;$; Charging voltage (total) [V]

$\mathrm{Nx}=20$; $\frac{\circ}{0}$ Number of spatial grid points on the transmission line

time $=400 e-9 ;$ 을

R_gen $=0.00165 /$ pfire; $\%$ Generator-side resistance [ohm]

C_gen $=(800 *$ pfire $) * 1 e-9 ; \%$ Generator-side capacitance [F]

$\mathrm{L}$ gen $=6 \mathrm{e}-9 / \mathrm{pfire} ;$ \% Generator-side inductance [H]

$\mathrm{R}$-core $=0.55$; $\%$ Equivalent resistance of the inductive cores [R]

$\mathrm{R}$ load $=.015 ;$ \% Resistance of the load [R]

gap $=1.3 e-2 ;$ Gap height $[\mathrm{m}]$

응 Current Trace Input

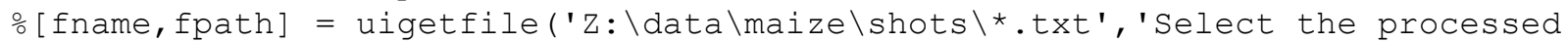
current trace');

oopenthis = [fpath, fname];

shotnum $=$ '00817';

CurrentTrace = dlmread (['Z: \data\maize\shots\' shotnum

' $\backslash$ traces_processed \BDot_2_Current.txt']);

Time = CurrentTrace $(:, 1 \overline{)}$; 


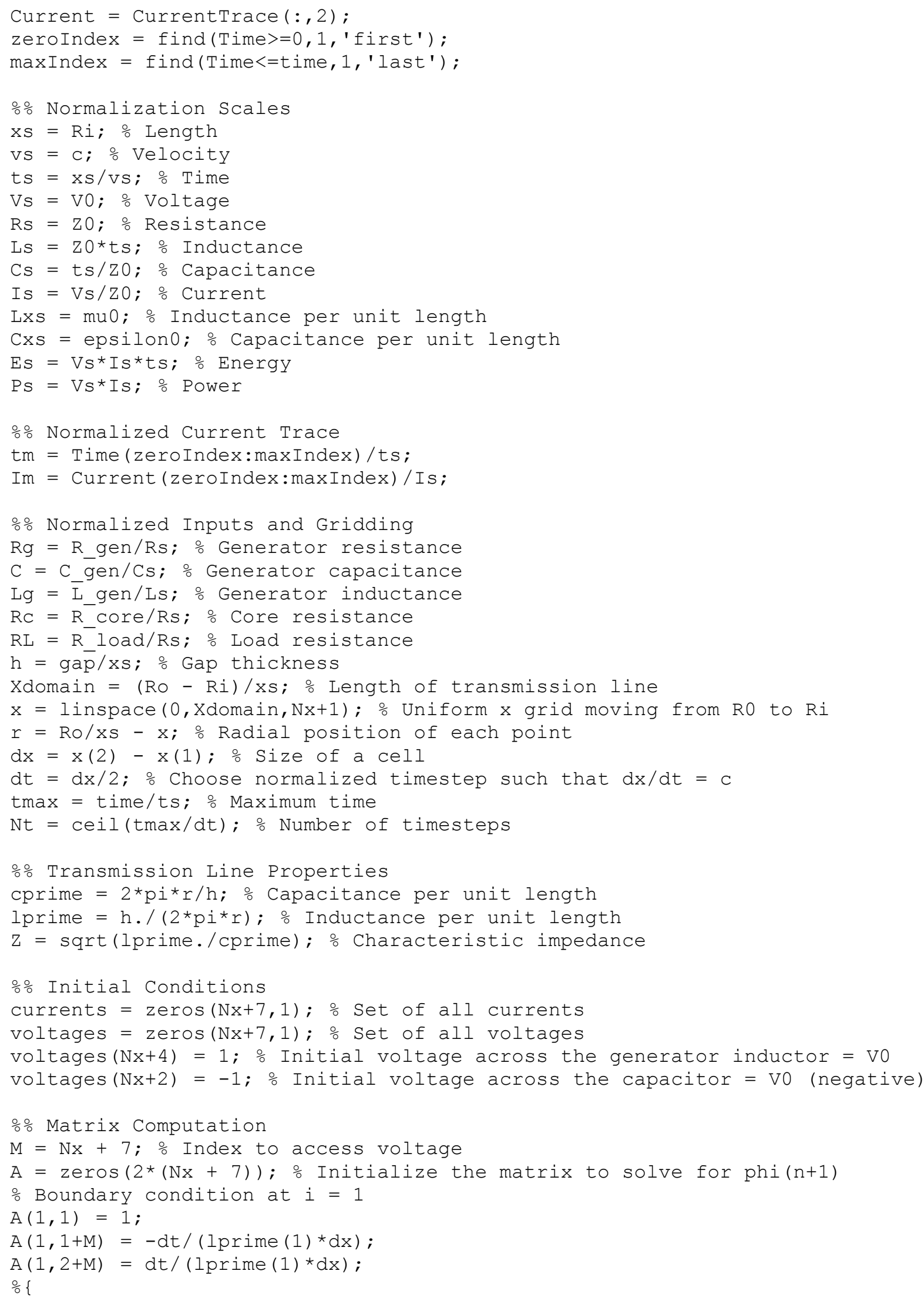


\% This section is currently replaced by the boundary condition on $V$ at 1 $\mathrm{A}(\mathrm{M}+1, \mathrm{M}+1)=1$;

$A(M+1,1)=-d t /(1$ prime $(1) * d x)$;

$\mathrm{A}(\mathrm{M}+1,2)=\mathrm{dt} /($ lprime $(1) * d x)$;

응

$\%$ Boundary condition at $i=\mathrm{Nx}+1$

응

\% This section is currently replaced by the boundary condition on I at $N x+1$

$\mathrm{A}(\mathrm{Nx}+1, \mathrm{Nx}+1)=1$;

$A(\mathrm{Nx}+1, \mathrm{Nx}+\mathrm{M})=-\mathrm{dt} /(\operatorname{cprime}(\mathrm{Nx}+1) * \mathrm{dx})$;

$\mathrm{A}(\mathrm{Nx}+1, \mathrm{Nx}+\mathrm{M}+1)=\mathrm{dt} /(\operatorname{cprime}(\mathrm{Nx}+1) * \mathrm{dx})$;

웅

$\mathrm{A}(\mathrm{Nx}+1+\mathrm{M}, \mathrm{Nx}+1+\mathrm{M})=1$;

$\mathrm{A}(\mathrm{Nx}+1+\mathrm{M}, \mathrm{Nx})=-\mathrm{dt} /(\mathrm{cprime}(\mathrm{Nx}+1) * \mathrm{dx})$;

$\mathrm{A}(\mathrm{Nx}+1+\mathrm{M}, \mathrm{Nx}+1)=\mathrm{dt} /(\operatorname{cprime}(\mathrm{Nx}+1) * \mathrm{dx})$;

\% All other points on the transmission line

for $i=2: \mathrm{Nx}$

$A(i, i)=1$;

$A(i, i+1+M)=d t /(\operatorname{lprime}(i) * 2 * d x)$;

$A(i, i-1+M)=-d t /(1$ prime $(i) * 2 * d x)$;

$A(i+M, i+M)=1$;

$A(i+M, i+1)=d t /(2 * \operatorname{cprime}(i) * d x)$;

end

$A(i+M, i-1)=-d t /\left(2{ }^{*} \operatorname{cprime}(i) * d x\right)$;

\% Capacitor $\mathrm{I}=\mathrm{C}^{\star} \mathrm{dV} / \mathrm{dt}$

$\mathrm{A}(\mathrm{Nx}+2+\mathrm{M}, \mathrm{Nx}+2+\mathrm{M})=1$;

$\mathrm{A}(\mathrm{Nx}+2+\mathrm{M}, \mathrm{Nx}+2)=-\mathrm{dt} / \mathrm{C}$;

을 Generator $\mathrm{V}=\mathrm{I} * \mathrm{R}$

$\mathrm{A}(\mathrm{Nx}+3+\mathrm{M}, \mathrm{Nx}+3+\mathrm{M})=1$;

$\mathrm{A}(\mathrm{Nx}+3+\mathrm{M}, \mathrm{Nx}+3)=-\mathrm{Rg}$;

o Kirchoff's Voltage Law on the generator side

$\mathrm{A}(\mathrm{Nx}+4+\mathrm{M}, \mathrm{Nx}+4+\mathrm{M})=1$;

$\mathrm{A}(\mathrm{Nx}+4+\mathrm{M}, \mathrm{Nx}+2+\mathrm{M})=1$;

$\mathrm{A}(\mathrm{Nx}+4+\mathrm{M}, \mathrm{Nx}+3+\mathrm{M})=1$;

$\mathrm{A}(\mathrm{Nx}+4+\mathrm{M}, \mathrm{Nx}+5+\mathrm{M})=1$;

을 $\mathrm{V}=\mathrm{I} * \mathrm{R}$

$\mathrm{A}(\mathrm{Nx}+5+\mathrm{M}, \mathrm{Nx}+5+\mathrm{M})=1$;

$\mathrm{A}(\mathrm{Nx}+5+\mathrm{M}, \mathrm{Nx}+5)=-\mathrm{RC}$;

Kirchoff's Voltage Law on the load side

$\mathrm{A}(\mathrm{Nx}+6+\mathrm{M}, \mathrm{Nx}+6+\mathrm{M})=1$;

$\mathrm{A}(\mathrm{Nx}+6+\mathrm{M}, \mathrm{Nx}+7+\mathrm{M})=1$;

$\mathrm{A}(\mathrm{Nx}+6+\mathrm{M}, \mathrm{Nx}+1+\mathrm{M})=-1$;

$\div$ Load $V=I * R$

$\mathrm{A}(\mathrm{Nx}+7+\mathrm{M}, \mathrm{Nx}+7+\mathrm{M})=1$;

$\mathrm{A}(\mathrm{Nx}+7+\mathrm{M}, \mathrm{Nx}+7)=-\mathrm{RL}$;

- Voltage continuity at the input node of the transmission line

$\mathrm{A}(1+\mathrm{M}, \mathrm{Nx}+5+\mathrm{M})=-1$;

$\mathrm{A}(1+\mathrm{M}, 1+\mathrm{M})=1$;

\% Current continuity at the output node of the transmission line

$\mathrm{A}(\mathrm{Nx}+1, \mathrm{Nx}+1)=1$;

ㅇ Current continuity at generator resistor

$A(N x+2, N x+2)=1$;

$\mathrm{A}(\mathrm{Nx}+2, \mathrm{Nx}+3)=-1$;

\% Current continuity at generator inductor

$\mathrm{A}(\mathrm{Nx}+3, \mathrm{Nx}+3)=1$;

$\mathrm{A}(\mathrm{Nx}+3, \mathrm{Nx}+4)=-1$; 


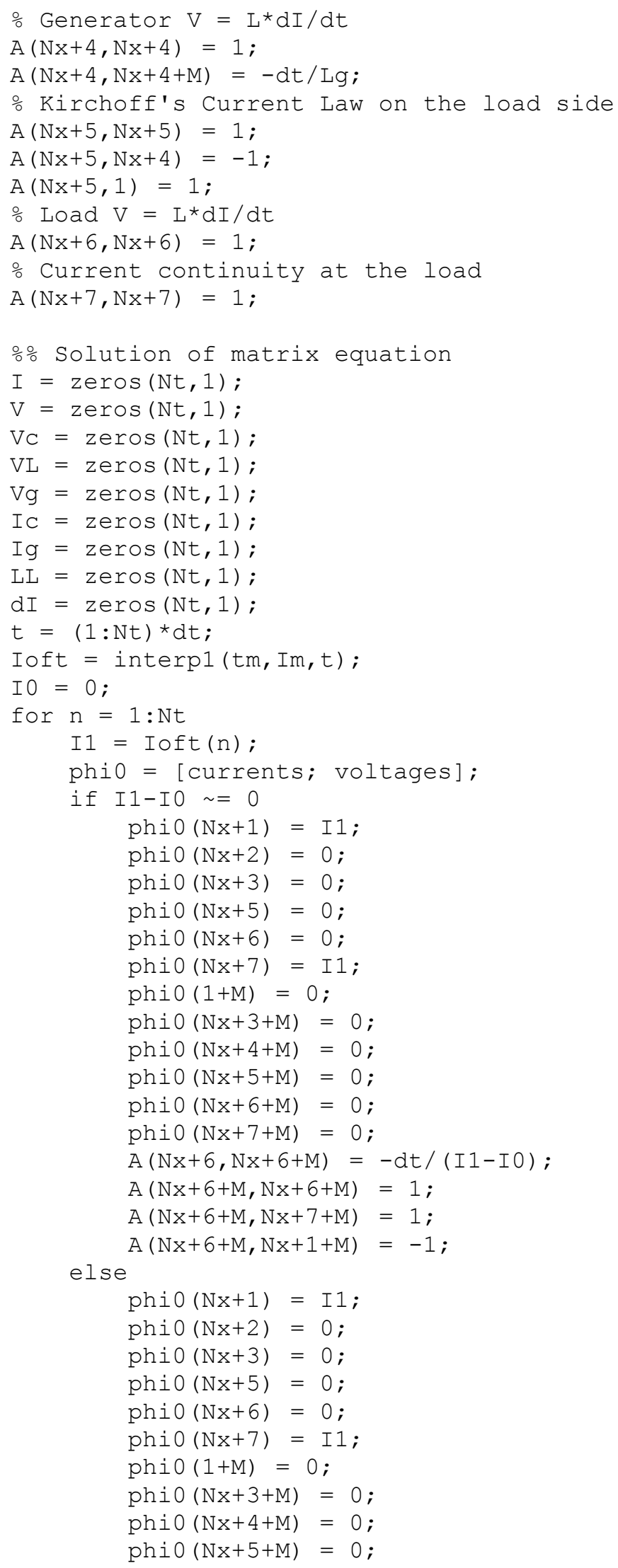




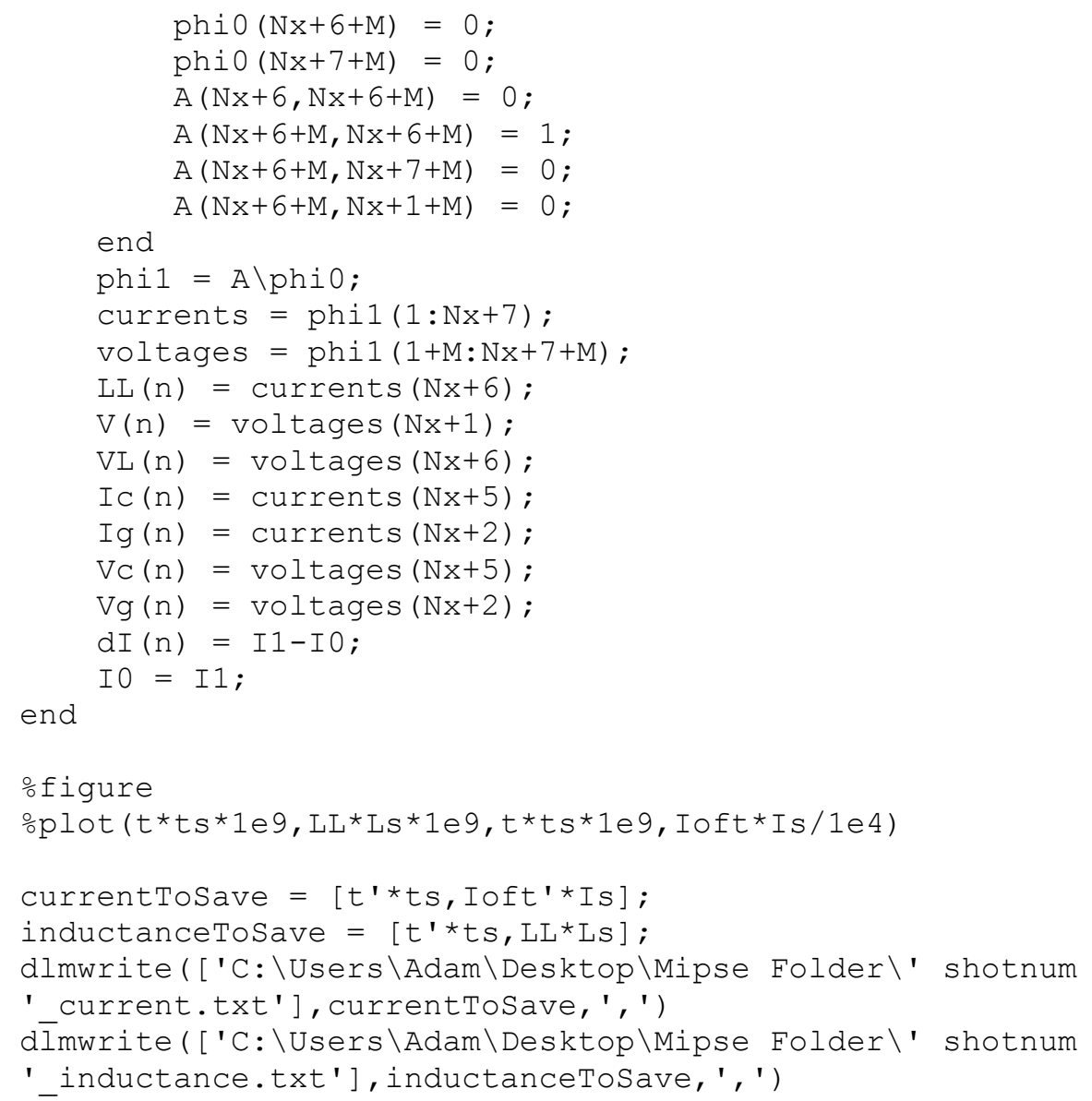




\section{APPENDIX C}

\section{Switch Description and Rebuild Procedure for MAIZE}

The LTD employs 40 six-gap sequence multi-electrode spark gap switches developed by HCEI in Tomsk, Russia. These switches consist of anode and cathode plates, five additional intermediate electrode stages, a plastic housing that holds the electrodes in place, and gas lines to allow for pressurization. The following figures show the components of a switch along with relevant dimensions:

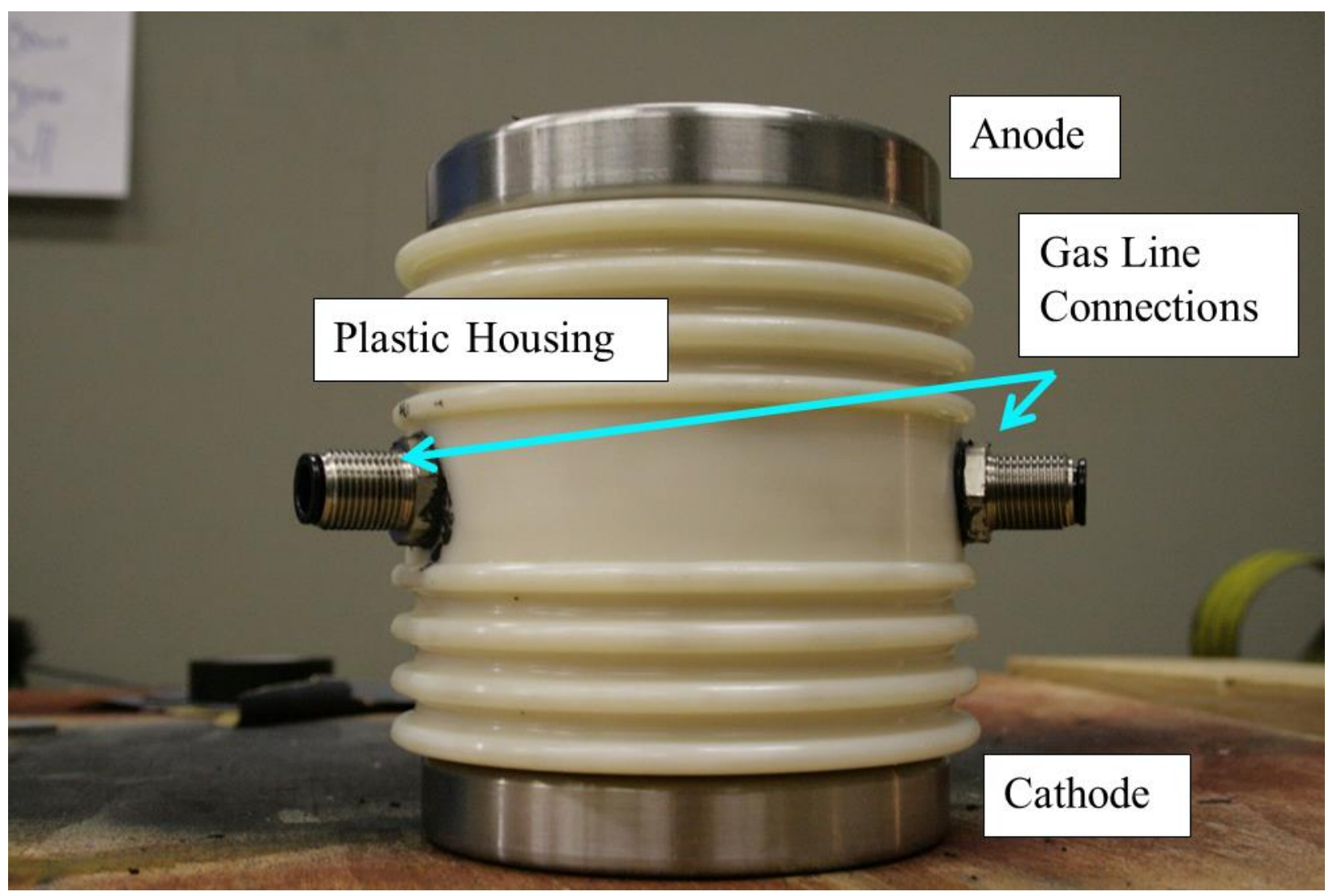

Figure C.2: Fully assembled switch exterior view 


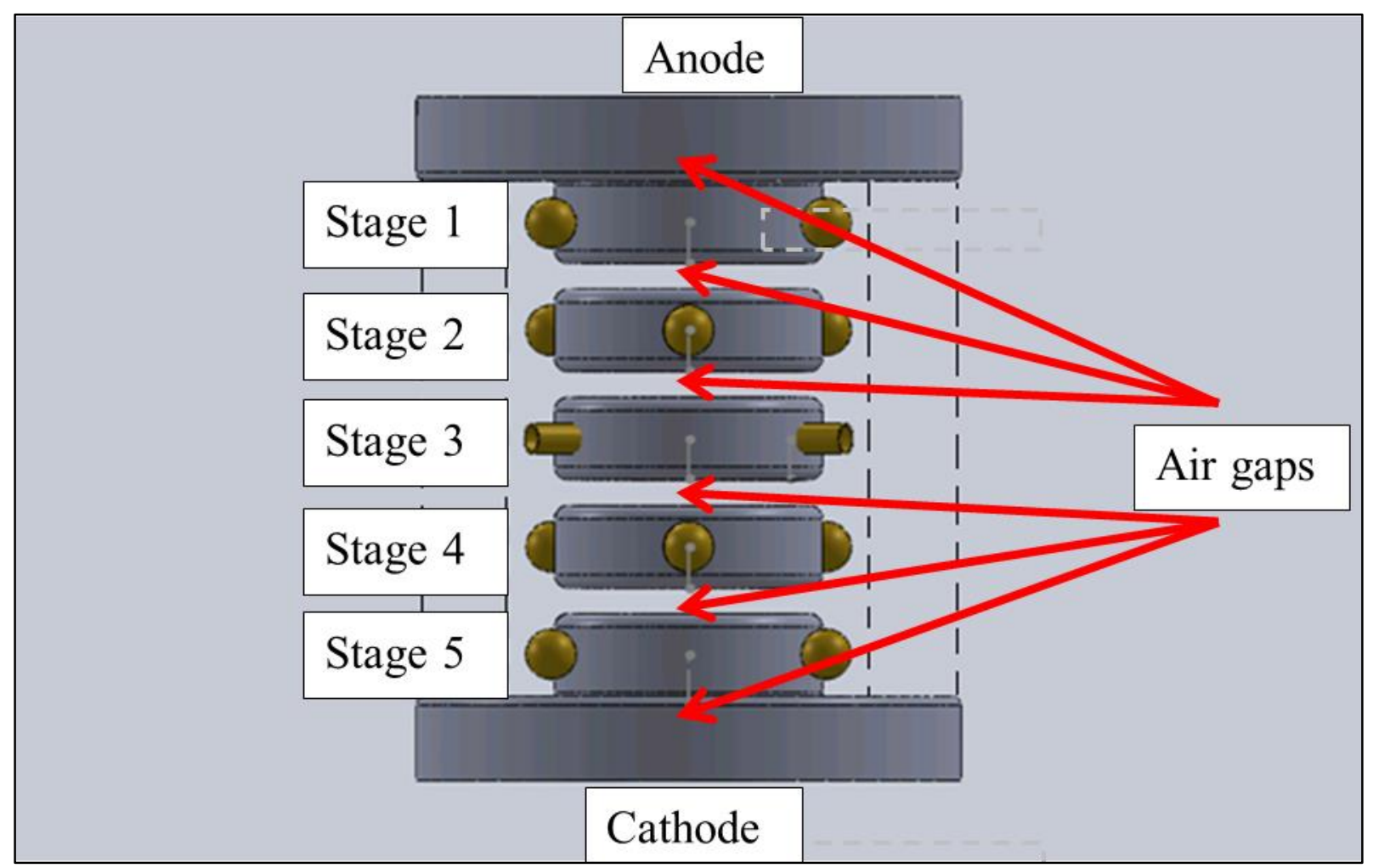

Figure C.2: Schematic of fully assembled switch interior view
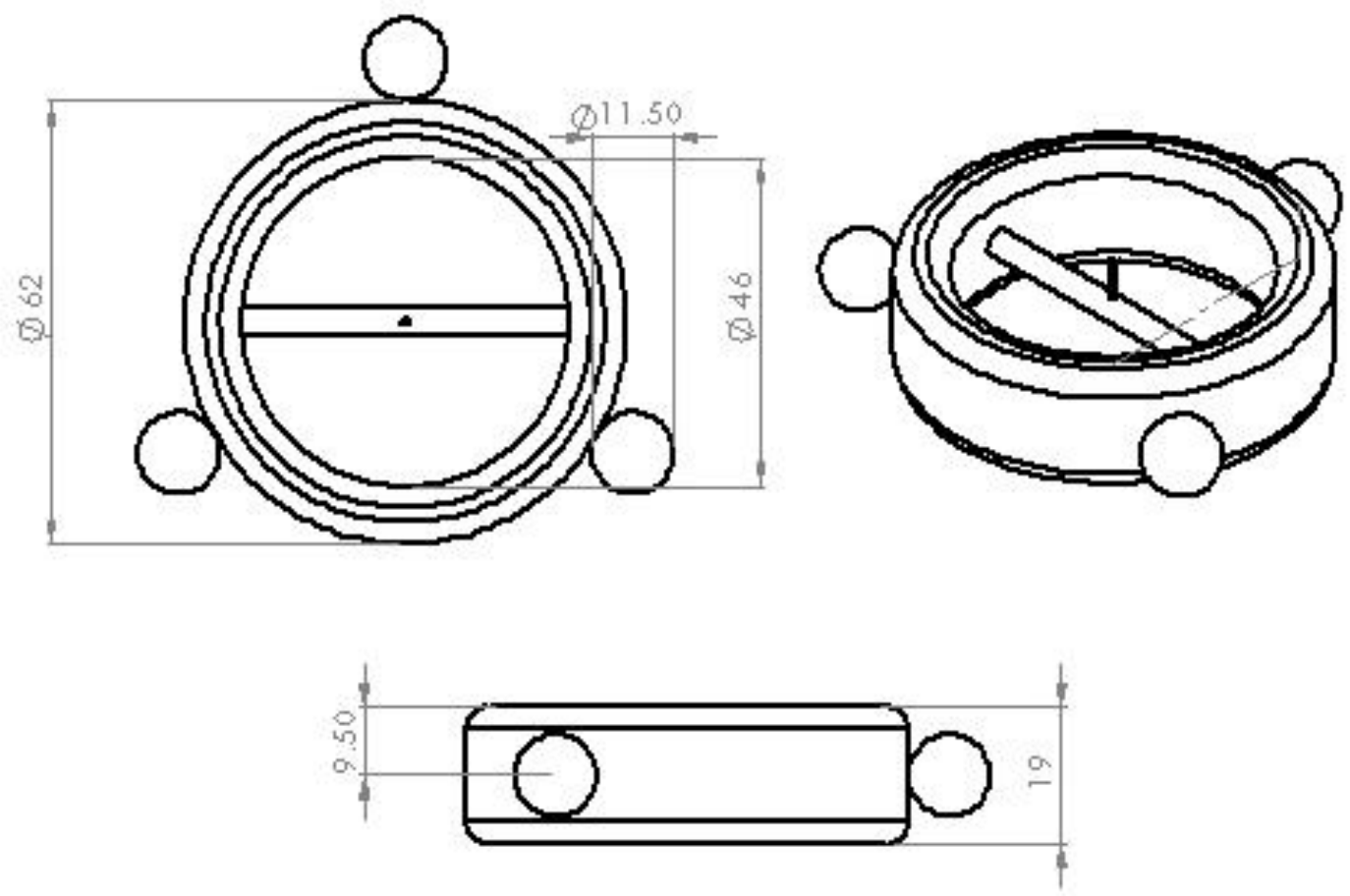

Figure C.3: Stage 2 and 4 electrode schematics 

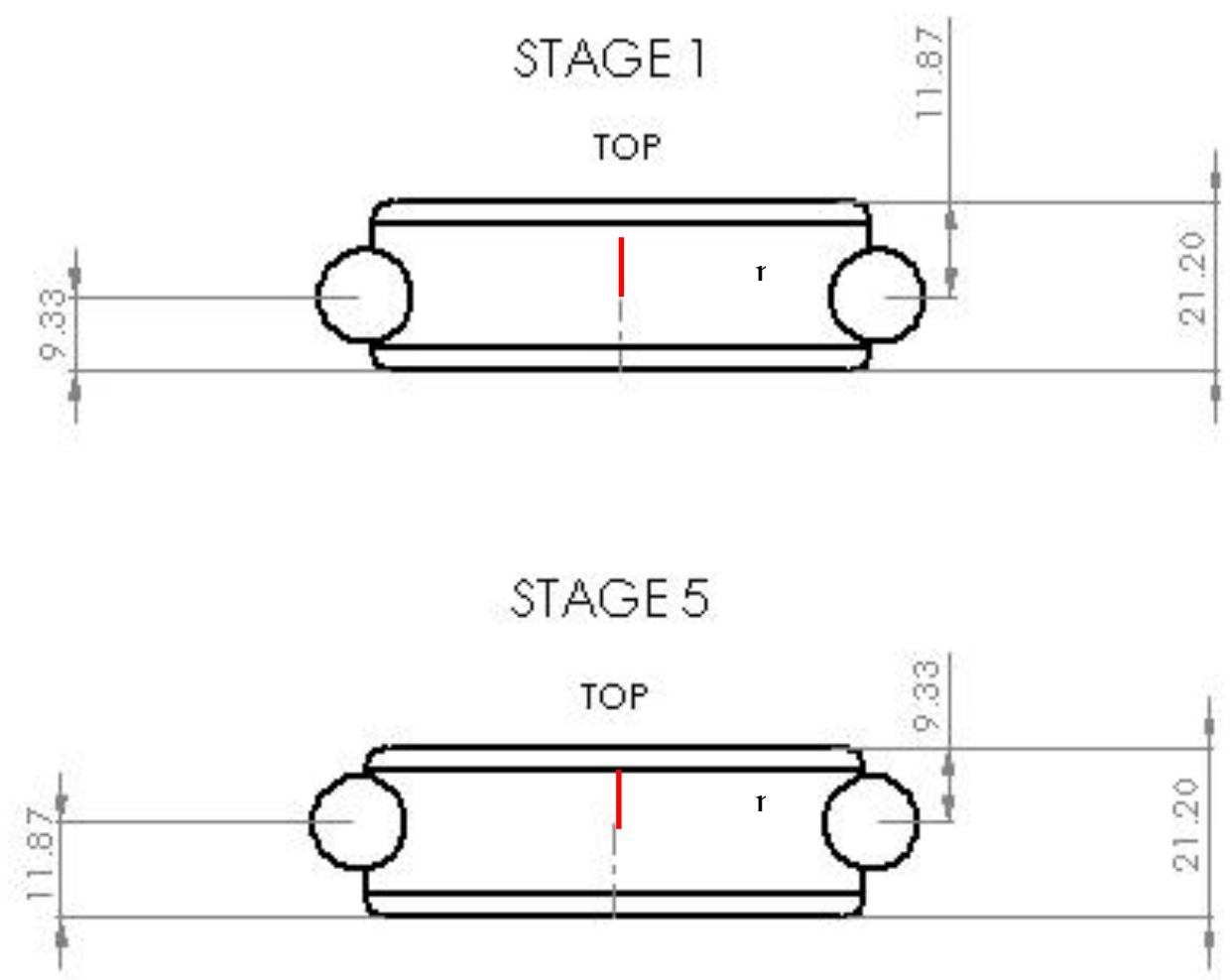

Figure C.4: Side view of stages 1 and 5
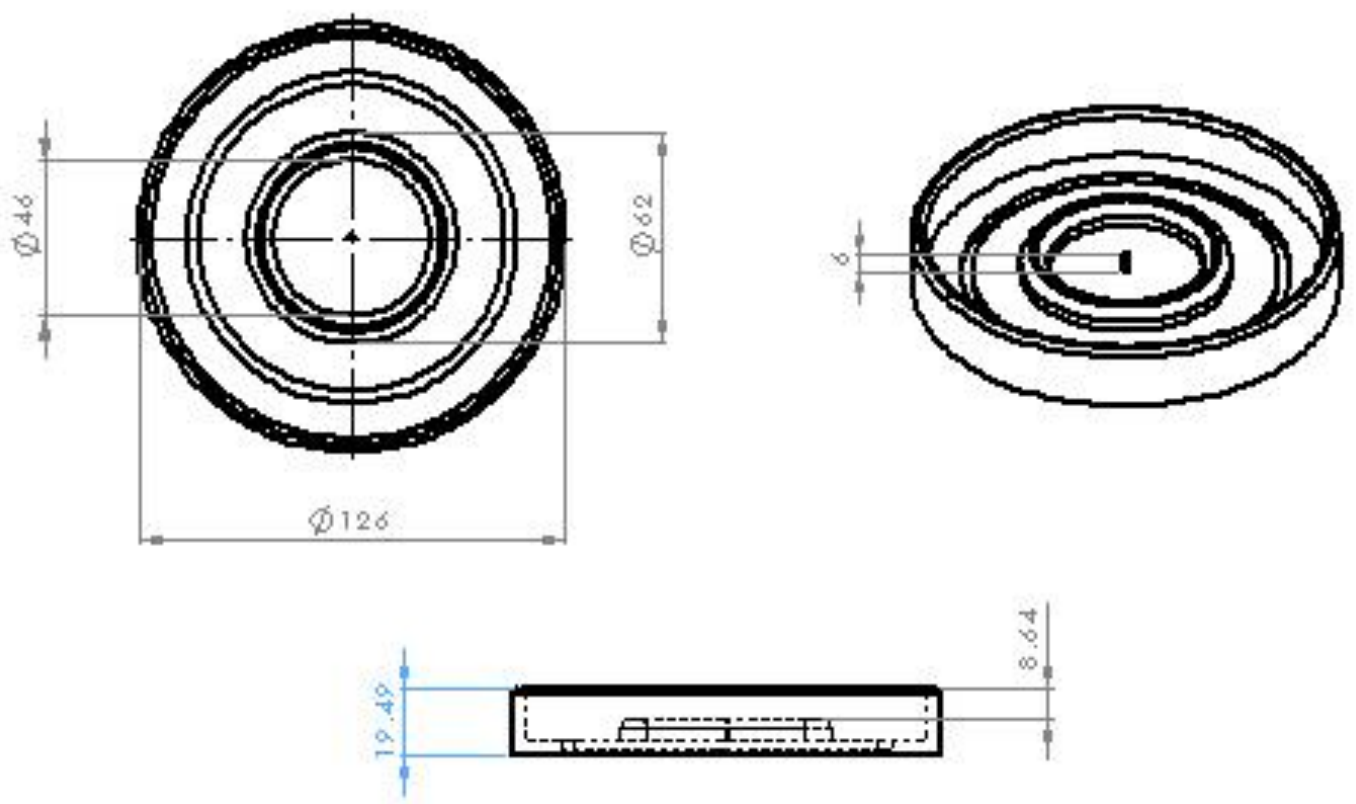

Figure C.5: Anode and cathode electrode plate schematics 
The brass balls on the intermediate stages are tapped with M6 threads, and a 20-mm M6 bolt is inserted through a blind hole in the electrode into the threads of each brass ball. The bolt is coated with a silicone adhesive such that turning the bolt head advances the brass ball away from the electrode. Grooves cut into the plastic switch housing receive the brass balls, holding each electrode in a fixed position. Stages 2, 3, and 4 are identical in size and bolt positioning except stage 3 has two of the brass balls replaced with tubes that mate with the external gas line connections. The external connections screw into threaded holes on the plastic housing and connect to $10-\mathrm{mm}$ OD gas lines via Legris press-release fittings. Silicone adhesive is used to create an airtight seal between the plastic housing and the threads. These external connections are electrically connected to stage 3 , allowing for a trigger or ground line to be connected to the middle electrode. Stages 1 and 5 are larger than the other three stages by $2.2 \mathrm{~mm}$, and the bolt holes are offset from center (upward on stage 5 and downward on stage 1) by approximately 1.25 $\mathrm{mm}$. The reason for this offset design is unknown, but when all stages are properly installed each air gap has a height of $6.0 \pm 0.3 \mathrm{~mm}$.

All electrodes except the anode plate have a needle pointing towards the anode side of the switch. This needle is soft soldered into a $2.7 \mathrm{~mm}$ stainless steel rod spanning the diameter on each intermediate stage. The cathode plate also has a needle soft soldered directly to the plate. Each needle protrudes $6 \mathrm{~mm}$ from the solder point and is sharpened to a point such that it resembles the tip of a hypodermic needle.

The anode and cathode plates screw onto either end of the plastic housing. An o-ring of ID $94.84 \mathrm{~mm}$ and width 3.53 (standard size number 2-240) makes a pressure seal when the electrode plates are tightened. 


\section{1 Normal Operation}

The switches are designed to hold off high voltage placed across the anode and cathode plates until a trigger pulse is delivered to the stage 3 (middle) electrode, at which point the switch nearly instantaneously breaks down and becomes an electrical short. The breakdown time varies depending on the input pulse but has been observed to be on order 100 ns. Dry air is used as the fill gas in the switch at a voltage-dependent pressure, typically 20 to 50 psig. Jitter is in general pressure dependent; for properly set pressures jitter is on order nanosecond.

On the MAIZE linear transformer driver, the switches operate at 26 psig for the standard charging voltage of $+/-70 \mathrm{kV}$. The switch/capacitor bricks are submerged in Univolt 60 mineral oil to prevent arcing, and the positive pressure in the switches prevents oil from leaking across the Legris fittings. Between shots, the switches are purged by exhausting air until the pressure drops to $\sim 5 \mathrm{psig}$, then bringing the switch back up to operating pressure. The purging process is repeated 5 to 10 times, which is sufficient to remove $>99 \%$ of breakdown products from the previous shot.

A switch is triggered by applying a voltage pulse to the center electrode. On the LTD, a trigger pulse of $70 \mathrm{kV}$ is supplied by the Russian Pail Generator (RPG). Other triggers, including a $100 \mathrm{kV}$ Maxwell generator, have been used to successfully trigger a switch. Triggering the switch drops its resistance to effectively zero and connects the capacitors of the brick in series, discharging their stored energy into the load.

\section{C.2 Sequence Multi-Electrode Breakdown Theory}

For a sequence multiple electrode switch to fire properly, the anode-cathode potential difference should be evenly distributed across the gaps. Usually, this is accomplished by connecting the stages with large (G $\Omega$-scale) resistors and dividing the voltage resistively. The 
HCEI switches do not include such resistors, so the voltage division is accomplished by the needles on the cathode side of each air gap. When the anode-cathode potential is applied to the switch, the sharp needle tip on the cathode sees a large local electric field, which causes field emission and partial coronal breakdown. Electrons liberated from the needle and the background gas strike the electrode adjacent to the cathode (electrode 5), giving rise to a small but finite current. The current causes a voltage drop across the gap. At this point, electrode 5 is biased negatively with respect to electrode 4 , so it also emits electrons, creating a resistive current channel and associated potential difference. This process continues until the entire switch has divided the applied A-K voltage evenly between the six gaps.

The charging voltage should apply a resistively divided potential across each gap that is significantly smaller than the self-breakdown voltage of the gap to avoid prefires. At $+/-70 \mathrm{kV}$ and 26 psig, the potential drop per gap is roughly half of the self-break threshold. A large trigger is therefore necessary to ensure rapid low-jitter breakdown. The $70 \mathrm{kV}$ pulse output by the RPG to the external connection on stage 3 of a switch rapidly changes the potential of stage 3 from 0 to $70 \mathrm{kV}$, which exceeds the self-break threshold of the 3-4 gap by a little more than a factor of two. When this gap breaks down, the 3 and 4 electrodes are shorted. This leads to a cascade event that shorts all of the electrodes and closes the switch.

\section{C.3 Switch rebuilding and testing}

In general, problems developed by the HCEI switches caused the LTD to prefire at low pressures; if the switch pressure is raised to prevent prefiring, an unacceptably high number of switches no-fire. The operating pressure was continuously raised to reduce the likelihood of prefire; by the time the LTD was taken down for rebuild, the operating pressure had been raised by about 15 psi to 40 psig. 
A fiber optic diagnostic was developed to measure switch closing timing. This diagnostic replaced one of the air lines with a Legris tubing containing a fiber optic, pointing the end of the fiber at the middle gap of the switch. Each fiber extends from the switch location in the LTD to the screen room through a 3/4-inch plastic gas line connected to one of the inlet ports of the LTD cavity. These fibers are connected to PMTs to determine the timing of switch closure. In normal switch operation, the switch becomes fully conductive around $40 \mathrm{~ns}$ after the trigger pulse reaches the midplane, with jitter on the order of $10 \mathrm{~ns}$. An example switch PMT signal trace indicating the relevant timings is shown in Figure C.6.

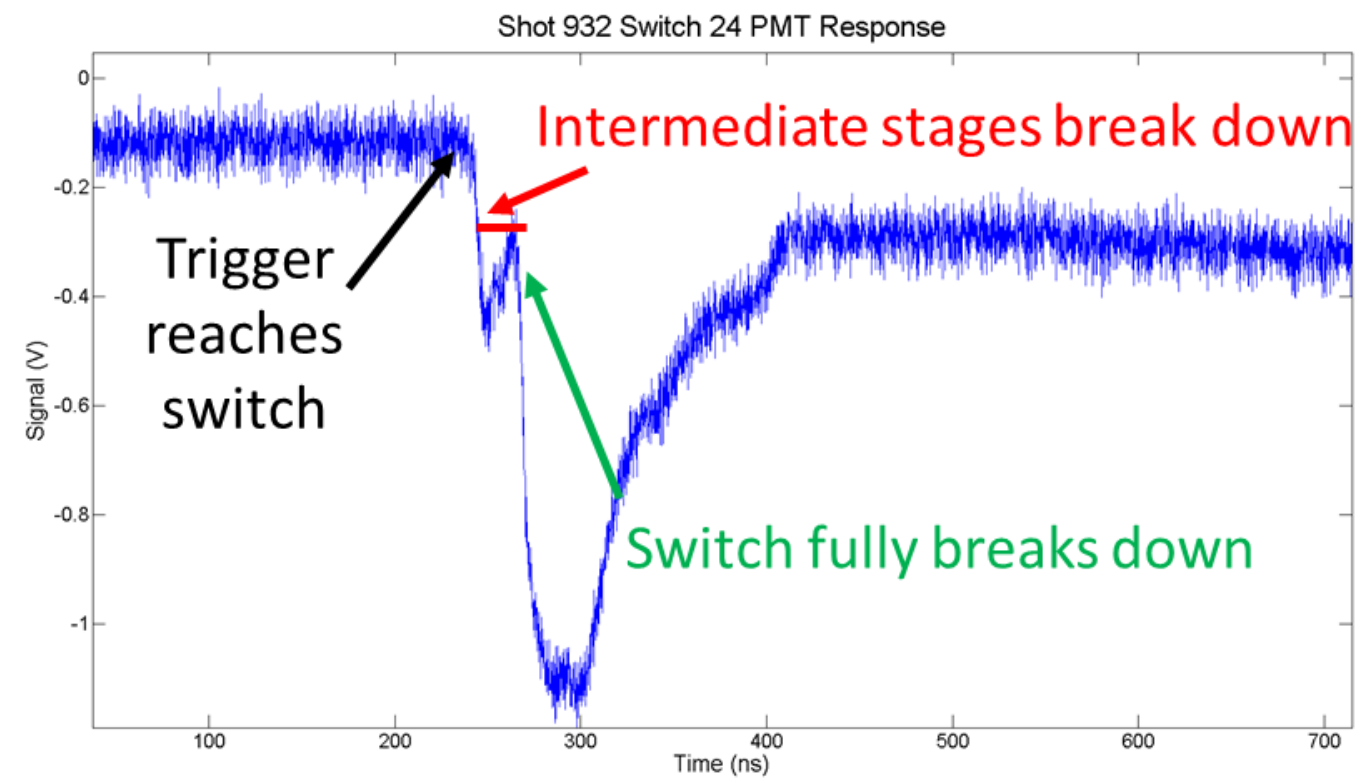

Figure C.6: PMT signal from a switch during the breakdown process.

PMT signals from a properly performing switch and a switch requiring rebuilding are compared in Figure C.7 and Figure C.8. Note the normally $10 \mathrm{~ns}$ jitter exhibited in Figure has increased to almost 200 ns in Figure. 


\section{Switch 28 Timings}

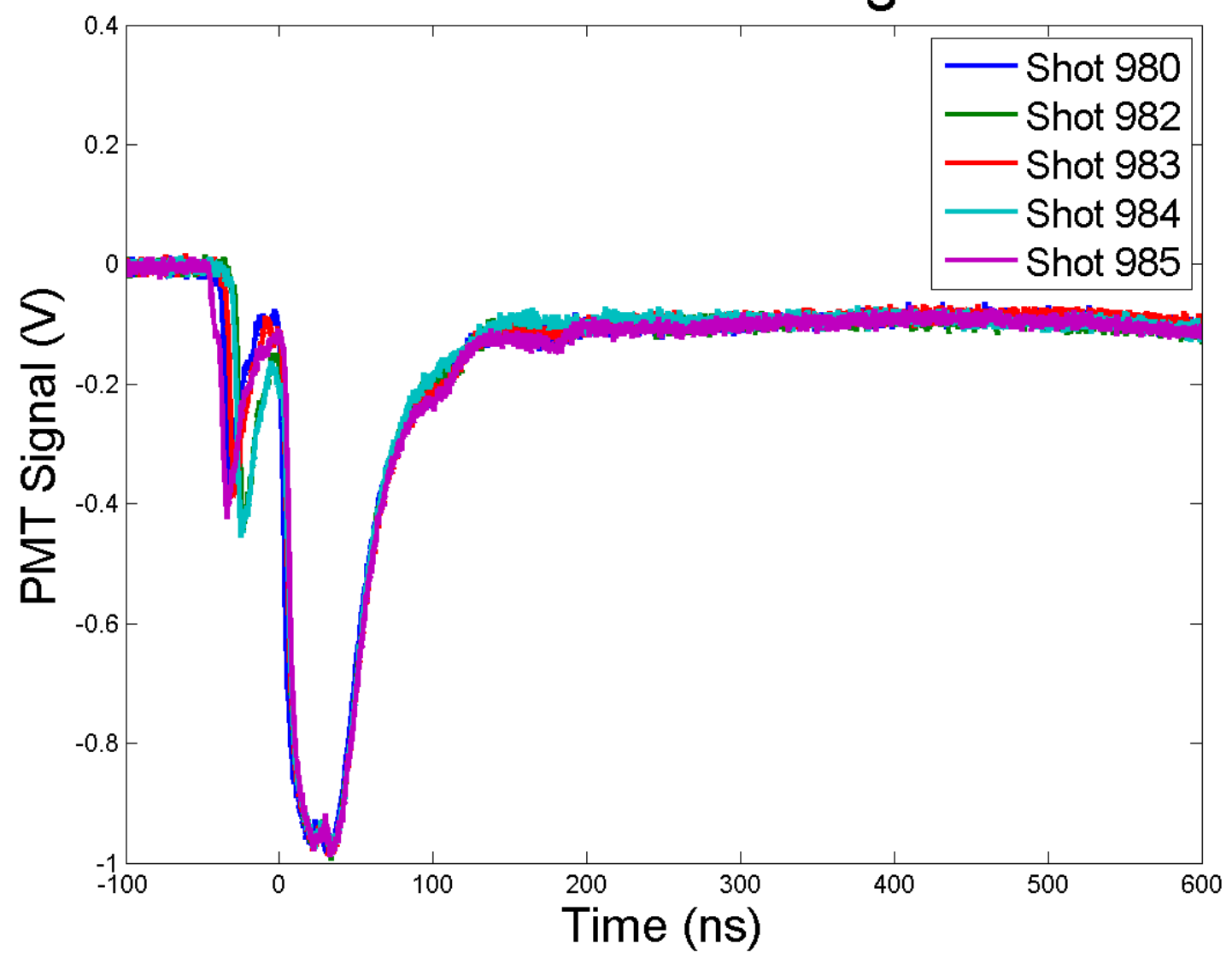

Figure C.7: PMT signals from a properly performing switch. Note the normal $10 \mathrm{~ns}$ jitter. 


\section{Switch 30 Timings}

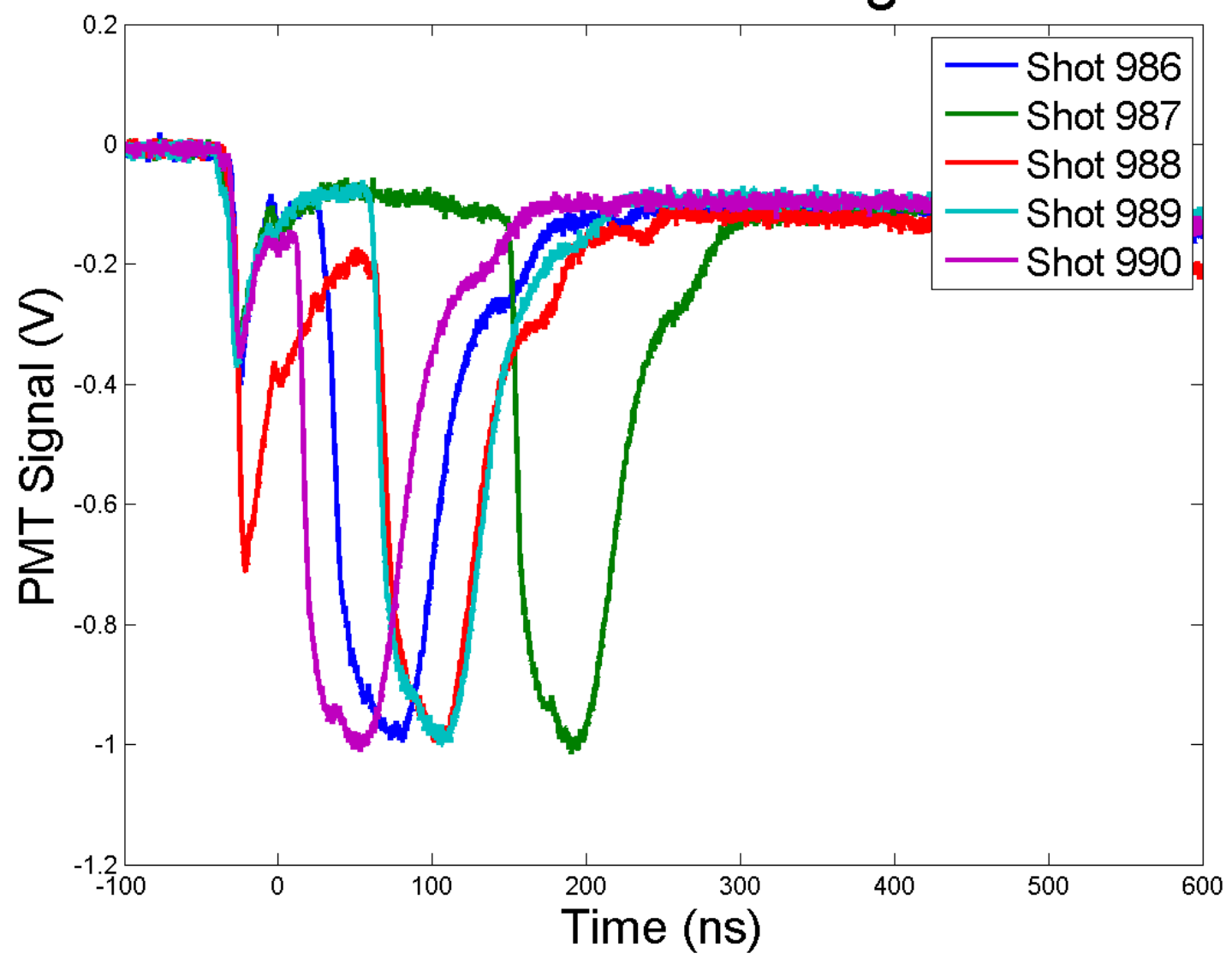

Figure C.8: PMT signals from a switch requiring rebuilding. Note the normally $10 \mathrm{~ns}$ jitter exhibited in Figure C.7 has increased to almost $200 \mathrm{~ns}$.

To rebuild a switch, the entire switch is first disassembled; the list of parts for each switch should include the anode plate, cathode plate, stage 1 through 5 electrode plates, two gas inlets, and the switch housing. Each component should be labeled to avoid confusion when reassembling the switch. Material residue and arc marks on every surface are removed by sanding, which is performed by securing the components on a lathe on a low speed setting ( 60 rpm) and holding sandpaper up to the surface while the part spins to achieve azimuthally uniform sanding. The internal surface of the plastic housing is sanded coarsely using $~ 250$ grit 
sandpaper. The housing is then submersed in an ultrasonic cleaner with a mild cleaning solution for around 10 minutes to remove oil and sand residue, rinsed in plain tap water, and allowed to air dry. All metal surfaces (except the gas feed lines) are sanded with a progression of grits to obtain a highly polished finish; the progression used on the fall 2015 rebuild was 240-600-10001500. After the electrodes are sanded, all metal components (including the gas feed lines) are submersed in the ultrasonic cleaner with a mild cleaning solution for 10 minutes. These components are then rinsed, first in tap water, then in isopropyl alcohol to accelerate evaporation. The components are air dried, then reattached to the switch body, starting with the trigger plane electrode. Threadings on the gas inlets, which also serve as the supports for the trigger plane electrode, are coated with silicone RTV prior to reattaching them to the switch to make an oil-tight seal at their interfaces. After the five trigger plane electrodes have been reattached, fresh o-rings are installed on the anode and cathode plates, which are threaded on using a strap wrench to achieve sufficient o-ring compression.

Reassembled switches are connected one at a time to a simple testing circuit to evaluate jitter and perform conditioning. The testing circuit consists of a single LTD brick, using two of the same $40 \mathrm{nF}, 100 \mathrm{kV}$ capacitors. The high voltage lines normally used to charge the LTD were connected to the anode and cathode of the switch through $1 \mathrm{k} \Omega$ resistors. A resistive (steady-state) ground is attached to one side of the switch. The same Maxwell generator used to trigger the LTD is connected to the trigger plane to fire the brick tester. The switch is conditioned by firing 100 times at $20 \mathrm{psig}$, then tested for prefires 10 times each at $25 \mathrm{psig}, 20$ psig, and $15 \mathrm{psig}$, and evaluated for late firing at $30 \mathrm{psig}$. The switch is deemed acceptable if no prefires occur at 25 psig and no more than 2 occur at 20 psig. 


\section{BIBLIOGRAPHY}

1. S. A. Slutz, M. C. Herrman, R. A. Vesey, A. B. Sefkow, D. B. Sinars, D. C. Rovang, K. J. Peterson, and M. E. Cuneo, Physics of Plasmas 17, 056303 (2010)

2. A. B. Sefkow, S. A. Slutz, J. M. Koning, M. M. Marinak, K. J. Peterson, D. B. Sinars, and R. A. Vesey, Physics of Plasmas 21, 072711 (2014)

3. S. A. Slutz and R. A. Vesey, Physical Review Letters 108, 025003 (2012)

4. R. D. McBride, M. R. Martin, R. W. Lemke, J. B. Greenly, C. A. Jennings, D. C. Rovang, D. B. Sinars, M. E. Cuneo, M. C. Herrmann, S. A. Slutz, C. W. Nakhleh, D. D. Ryutov, J. P. Davis, D. G. Flicker, B. E. Blue, K. Tomlinson, D. Schroen, R. M. Stamm, G. E. Smith, J. K. Moore, T. J. Rogers, G. K. Robertson, R. J. Kamm, I. C. Smith, M. Savage, W. A. Stygar, G. A. Rochau, M. Jones, M. R. Lopez, J. L. Porter, and M. K. Matzen, Physics of Plasmas 20, 056309 (2013)

5. M. R. Gomez, S. A. Slutz, A. B. Sefkow, D. B. Sinars, K. D. Hahn, S. B. Hansen, E. C. Harding, P. F. Knapp, P. F. Schmidt, C. A. Jennings, T. J. Awe, M. Geissel, D. C. Rovang, G. A. Chandler, G. W. Cooper, M. E. Cuneo, A. J. Harvey-Thompson, M. C. Herrmann, M. H. Hess, O. Johns, D. C. Lamppa, M. R. Martin, R. D. McBride, K. J. Peterson, J. L. Porter, G. K. Robertson, G. A. Rochau, C. L. Ruiz, M. E. Savage, I. C. Smith, W. A. Stygar, and R. A. Vesey, Physical Review Letters 113, 155003 (2014)

6. T. J. Awe, K. J. Peterson, E. P. Yu, R. D. McBride, D. B. Sinars, M. R. Gomez, C. A. Jennings, M. R. Martin, S. E. Rosenthal, D. G. Schroen, A. B. Sefkow, S. A. Slutz, K. Tomlinson, and R. A. Vesey, Physical Review Letters 116, 065001 (2016)

7. W. A. Stygar, M. E. Cuneo, D. I. Headley, H. C. Ives, R. J. Leeper, M. G. Mazarakis, C. L. Olson, J. L. Porter, T. C. Wagoner, and J. R. Woodworth, Physical Review Special Topics-Accelerators and Beams 10, 030401 (2007)

8. W.A. Stygar, W.E. Fowler, K.R. LeChien, F.W. Long, M.G. Mazarakis, G.R. McKee, J.L. McKenney, J.L. Porter, M.E. Savage, B.S. Stoltzfus, D.M. Van De Valde, and J.R. Woodworth, Phys. Rev. ST Accel. Beams 12, 030402 (2009)

9. W.A. Stygar, T.J. Awe, J.E. Bailey, N.L. Bennett, E.W. Breden, E.M. Campbell, R.E. Clark, R.A. Cooper, M.E. Cuneo, J.B. Ennis, D.L. Fehl, T.C. Genoni, M.R. Gomez, G.W. 
Greiser, F.R. Gruner, M.C. Herrmann, B.T. Hutsel, C.A. Jennings, D.O. Jobe, B.M. Jones, M.C. Jones, P.A. Jones, P.F. Knapp, J.S. Lash, K.R. LeChien, J.J. Leckbee, R.J. Leeper, S.A. Lewis, F.W. Long, D.J. Lucero, E.A. Madrid, M.R. Martin, M.K. Matzen, M.G. Mazarakis, R.D. McBride, G.R. McKee, C.L. Miller, J.K. Moore, C.B. Mostrom, T.D. Mulville, K.J. Peterson, J.L. Porter, D.B. Reisman, G.A. Rochau, G.E. Rochau, D.V. Rose, D.C. Rovang, M.E. Savage, M.E. Sceiford, P.F. Schmit, R.F. Schneider, J.

Schwarz, A.B. Sefkow, D.B. Sinars, S.A. Slutz, R.B. Spielman, B.S. Stoltzfus, C. Thoma, R.A. Vesey, P.E. Wakeland, D.R. Welch, M.L. Wisher, and J.R. Woodworth, Phys. Rev ST Accel. Beams 18, 110401 (2015)

10. S. A. Slutz, W. A. Stygar, M. R. Gomez, K. J. Peterson, A. B. Sefkow, D. B. Sinars, R. A. Vesey, E. M. Campbell, and R. Betti

11. K. J. Peterson, D. B. Sinars, E. P. Yu, M. C. Herrmann, M. E. Cuneo, S. A. Slutz, I. C. Smith, B. W. Atherton, M. D. Knudson, and C. Nakhleh, Physics of Plasmas 19, 092701 (2012)

12. A. H. Nelson and M. G. Haines, Plasma Physics 11, 811 (1969)

13. M. G. Haines, Journal of Plasma Physics 12, 1 (1974)

14. G. B. Field, Astrophysical Journal 142, 531 (1965)

15. I. Aranson, B. Meerson, and P. V. Sasarov, Physical Review E 47, 4337 (1993)

16. M. Sadowski, H. Herold, H. Schmidt, and M. Shakhatre, Physics Letters 105A, 117 (1984)

17. M. A. Liberman, J. S. De Groot, A. Toor, and R. Spielman, Physics of high-density Zpinch plasmas (Springer, 1999)

18. D. B. Sinars, T. A. Shelkovenko, S. A. Pikuz, M. Hu, V. M. Romanova, K. M. Chandler, J. B. Greenly, D. A. Hammer, and B. R. Kusse, Physics of Plasmas Letters 7, 429 (2000)

19. V. I. Oreshkin, Physics of Plasmas 15, 092103 (2008)

20. A. G. Rousskikh, V. I. Oreshkin, S. A. Chaikovsky, N. A. Labetskaya, A. V. Shishlov, I. I. Beilis, and R. B. Baksht, Physics of Plasmas 15, 102706 (2008)

21. S. A. Chaikovsky, V. I. Oreshkin, G. A. Mesyats, N. A. Ratakhin, I. M. Datsko, and B. A. Kablambaev, Physics of Plasmas 16, 042701 (2009)

22. W. G. Chace and H. K. Moore, eds., Exploding Wires, Based on Conference on the Exploding Wire Phenomenon (1959) 
23. K. J. Peterson, E. P. Yu, D. B. Sinars, M. E. Cuneo, S. A. Slutz, J. M. Koning, M. M. Marinak, C. Nakhleh, and Mark. C. Herrmann, Physics of Plasmas 20, 056305 (2013)

24. K. J. Peterson, T. J. Awe, E. P. Yu, D. B. Sinars, E. S. Field, M. E. Cuneo, M. C. Herrmann, M. Savage, D. Schroen, K. Tomlinson, and C. Nakhleh, Physical Review Letters 112, 135002 (2014)

25. D. D. Ryutov, M. S. Derzon, and M. K. Matzen, Reviews of Modern Physics 72, 167 (2000)

26. G. R. Gathers, International Journal of Thermophysics 4, 209 (1983)

27. E. H. Buyco and F. E. Davis, Journal of Chemical Engineering Data 15, 518 (1970)

28. P. D. Desai, H. M. James, and C. Y. Ho, Journal of Physical and Chemical Reference Data 13, 1131 (1984)

29. E. A. Brandes and G. B. Brook, eds., Smithells Metals Reference Book $7^{\text {th }}$ Edition (1992)

30. B. Giordanengo, N. Benazzi, J. Vinckel, J. G. Gasser, and L. Roubi, Journal of NonCrystalline Solids 250-252, 377 (1999)

31. D. H. Menzel, ed., Fundamental Formulas of Physics (1953)

32. G. Antonini, A. Orlandi, and C. R. Paul, IEEE Transactions on Microwave Theory and Techniques 47, 979 (1999)

33. M. Riches (Invisible Vision Ltd.), personal communication (2016)

34. OMEGA Engineering, Table of Total Emissivity (2016)

35. S. Krishnan and P. C. Nordine, Physical Review B 47, 780 (1993)

36. Thomas Awe (Sandia National Laboratories), personal communication (2016)

37. D. A. Yager-Elorriaga, A. M. Steiner, S. G. Patel, N. M. Jordan, Y. Y. Lau, and R. M. Gilgenbach, Review of Scientific Instruments 86, 113506 (2015)

38. M. R. Weis, Magneto-Rayleigh-Taylor Instabilitiy: Theory and Simulation in Planar and Cylindrical Pulsed Power Targets, PhD dissertation, University of Michigan (2015)

39. M. R. Weis, P. Zhang, Y. Y. Lau, P. F. Schmidt, K. J. Peterson, H. Hess, and R. M. Gilgenbach, Physics of Plasmas 22, 032706 (2015)

40. T. C. Wagoner, W. A. Stygar, H. C. Ives, T. L. Gilliland, R. B. Spielman, M. F. Johnson, P. G. Reynolds, J. K. Moore, R. L. Mourning, D. L. Fehl, K. E. Androlewicz, J. E. Bailey, 
R. S. Broyles, T. A. Dinwoodie, G. L. Donovan, M. E. Dudley, K. D. Hahn, A. A. Kim, J. R. Lee, R. J. Leeper, G. T. Leifeste, J. A. Melville, J. A. Mills, L. P. Mix, W. B. S. Moore, B. P. Peyton, J. L. Porter, G. A. Rochau, G. E. Rochau, M. E. Savage, J. F. Seamen, J. D. Serrano, A. W. Sharpe, R. W. Shoup, J. S. Slopek, C. S. Speas, K. W. Struve, D. M. Van De Valde, and R. M. Woodring, Physical Review Special Topics-Accelerators and Beams 11, $100401(2008)$

41. M. R. Gomez, Experimental Examination of Plasma Formation and Current Loss in PostHole Convolutes, PhD Dissertation, University of Michigan (2011)

42. J. C. Zier, Ablation Dynamics and Instabilities of Metallic Plasmas Generated using MegaAmpere-Scale Current Drivers, PhD Dissertation, University of Michigan (2011)

43. D. A. Yager-Elorriaga, P. Zhang, A. M. Steiner, N. M. Jordan, Y. Y. Lau, and R. M. Gilgenbach, Physics of Plasmas, accepted and tentatively scheduled for publication in September 2016

44. A. A. Kim, M. G. Mazarakis, V. A. Sinebryukhov, B. M. Kovalchuk, V. A. Visir, S. N. Volkov, F. Bayol, A. N. Bastrikov, V. G. Durakov, S. F. Frolov, V. M. Alexeenko, D. H. McDaniel, W. E. Fowler, K. LeChien, C. Olson, W. A. Stygar, K. W. Struve, J. Porter, and R. M. Gilgenbach, Physical Review Special Topics-Accelerators and Beams 12, 050402 (2009)

45. M. G. Mazarakis, W. E. Fowler, A. A. Kim, V. A. Sinebryukhov, S. T. Rogowski, R. A. Sharpe, D. H. McDaniel, C. L. Olson, J. L. Porter, K. W. Struve, W. A. Stygar, and J. R. Woodworth, Physical Review Special Topics-Accelerators and Beams 12, 050401 (2009)

46. R. M. Gilgenbach, M. R. Gomez, J. C. Zier, W. W. Tang, D. M. French, Y. Y. Lau, M. G. Mazarakis, M. E. Cuneo, M. D. Johnston, B. V. Oliver, T. A. Melhorn, A. A. Kim, and V. A. Sinebryukhov, American Institute of Physics Conference Proceedings 1088, 259 (2009)

47. A. M. Steiner, D. A. Yager-Elorriaga, S. G. Patel, N. M. Jordan, R. M. Gilgenbach, A. A. Safronova, V. L. Kantsyrev, V. V. Shlyaptseva, I. Shrestha, and M. T. Schmidt-Petersen, Physics of Plasmas, accepted and tentatively scheduled for publication in September 2016

48. J. R. Woodworth, J. A. Alexander, F. R. Gruner, W. A. Stygar, M. J. Harden, J. R. Blickem, G. J. Dension, F. E. White, L. M. Lucero, H. D. Anderson, L. F. Bennett, S. F. Glover, D. Van DeValde, and M. D. Mazarakis, Physical Review Special TopicsAccelerators and Beams 12, 060401 (2009) 
49. J. C. Zier, R. M. Gilgenbach, D. A. Chalenski, Y. Y. Lau, D. M. French, M. R. Gomez, S. G. Patel, I. M. Rittersdorf, A. M. Steiner, M. Weis, P. Zhang, M. Mazarakis, M. E. Cuneo, and M. Lopez, Physics of Plasmas 19, 032701 (2012)

50. M. G. Mazarakis, W. E. Fowler, K. L. LeChien, F. W. Long, M. K. Matzen, D. H. McDaniel, R.G.McKee, C. L. Olson, J. L. Porter, S. T. Rogowski, K. W. Struve, W. A. Stygar, J. R. Woodworth, A. A. Kim, V. A. Sinebryukhov, R. M. Gilgenbach, M. R. Gomez, D. M. French, Y. Y. Lau, J. C. Zier, D. M. VanDevalde, R. A. Sharpe, and K. Ward, IEEE Transactions on Plasma Science 38, 704 (2010)

51. A. A. Kim, M. G. Mazarakis, V. I. Manylov, V. A. Vizir, and W. A. Stygar, Phys. Rev. ST Accel. Beams 13, 070401 (2010)

52. A. S. Safronova, V. L. Kantsyrev, M. E. Weller, V. V. Shlyaptseva, I. K. Shrestha, M. Y. Lorance, M. T. Schmidt-Petersen, A. Stafford, M. C. Cooper, A. M. Steiner, D. A. YagerElorriaga, S. G. Patel, N. M. Jordan, R. M. Gilgenbach, and A. S. Chuvatin, IEEE Transactions on Plasma Science 44, 432 (2016)

53. E. M. Waisman, M. E. Cuneo, W. A. Stygar, R. W. Lemke, K. W. Struve, and T. C. Wagoner, Physics of Plasmas 11, 2009 (2004)

54. D. P. Murphy, R. J. Allen, B. V. Weber, R. J. Commisso, J. P. Apruzese, D. G. Phipps, and D. Mosher, Review of Scientific Instruments 79, 10E306 (2008)

55. R. J. Commisso, J. P. Apruzese, J. Davis, S. L. Jackson, D. Mosher, D. P. Murphy, J. W. Thornhill, A. L. Velikovich, B. V. Weber, F. C. Young, J. S. Levine, B. H. Failor, H. Sze, N. Qi, J. W. Barrister, P. L. Coleman, K. Wilson, M. Krishnan, C. A. Coverdale, B. Jones, and C. Deeney, 7th International Conference on Dense Z-pinches, AIP Proceedings 1088, 233 (2009)

56. M. Engelhardt, Ltspice, Linear Technology Corporation (2010)

57. V. L. Kantsyrev, A. S. Safronova, D. A. Fedin, V. V. Ivanov, A. A. Esaulov, V. Nalajala, I. Shrestha, S. Pokala, K. Williamson, N. D. Ouart, M. F. Yilmaz, P. Laca, T. E. Cowan, L. I. Rudakov, B. Jones, C. A. Coverdale, C. Deeney, P. D. LePell, A. L. Velikovich, and A. S. Chuvatin, IEEE Transactions on Plasma Science 34, 194 (2006)

58. A. S. Safronova, V. L. Kantsyrev, A. A. Esaulov, N. D. Ouart, M. F. Yilmaz, K. M. Williamson, V. Shlyaptseva, I. Shrestha, G. C. Osborne, C. A. Coverdale, B. Jones, and C. Deeney, Review of Scientific Instruments 79, 10E315 (2008)

59. M. E. Weller, A. S. Safronova, V. L. Kantysrev, A. A. Esaulov, I. Shrestha, J. P. Apruzese, J. L. Giuliani, A. S. Chuvatin, A. Stafford, S. F. Keim, V. V. Shlyaptseva, G. C. Osborne, and E. E. Petkov, Physics of Plasmas 21, 031206 (2014) 
60. R. B. Baksht, S. I. Tkachenko, V. M. Romanova, A. R. Mingaleev, V. I. Oreshkin, A. E. Ter-Oganes'yan, T. A> Khattotov, T. A. Shelkovenko, and S. A. Pikuz, Technical Physics 58, 1129 (2013)

61. R. B. Baksht, A. G. Rousskikh, A. S. Zhigalin, V. I. Oreshkin, and A. P. Artyomov, Physics of Plasmas 22, 103521 (2015)

62. T. J. Awe, Electrothermal Instability Evolution on Z-Pinch Rods and Imploding Liners Pulsed with Intense Current, Presented at the $43^{\text {rd }}$ IEEE International Conference on Plasma Science (2016)

63. Prism Computational Sciences Inc., PrismSPECT (1998)

64. Sonal Patel (Sandia National Laboratories), personal communication (2016)

65. H.-K. Chung, M. H. Chen, W. L. Morgan, Y. Ralchenko and R. W. Lee, High Energy Density Physics 1, 3 (2005)

66. H. W. Liepmann and A. Roshko, Elements of Gas Dynamics, Wiley, New York (1957)

67. Y. B. Zel'dovich and Y. P. Raizer, Physics of Shock Waves and High Temperature Hydrodynamic Phenomena, Academic Press, New York (1966)

68. P. L. G. Ventzek, The Hydrodynamics of Excimer Laser Ablation Processing of Materials in Vacuum and Gases, PhD dissertation, University of Michigan (1991)

69. C. H. Ching, Dynamics of Laser Ablation in Gaseous and Plasma Environments, $\mathrm{PhD}$ dissertation, University of Michigan (1994)

70. V. Osterman and H. Antes, eds., Critical Melting Points and Reference Data for Vacuum Heat Treating (2010)

71. V. S. Sedoi, G. A. Mesyats, V. I. Oreshkin, V. V. Valevich, and L. I. Chemezova, IEEE Transactions on Plasma Science 27, 846 (1999)

72. M. D. Johnston, Ionization Dynamics of a Single Wire Z-Pinch, $\mathrm{PhD}$ dissertation, University of Michigan (2004)

73. M. E. Cuneo, M. C. Herrmann, D. B. Sinars, S. A. Slutz, W. A. Stygar, R. A. Vesey, A. B. Sefkow, G. A. Rochau, G. A. Chandler, J. E. Bailey, J. L. Porter, R. D. McBride, D. C. Rovang, M. G. Mazarakis, E. P. Yu, D. C. Lamppa, K. J. Peterson, C. Nakhleh, S. B. Hansen, A. J. Lopez, M. E. Savage, C. A. Jennings, M. R. Martin, R. W. Lemke, B. W. Atherton, I. C. Smith, P. K. Rambo, M. Jones, M. R. Lopez, P. J. Christenson, M. A. Sweeney, B. Jones, L. A. McPherson, E. Harding, M. R. Gomez, P. F. Knapp, T. J. Awe, R. J. Leeper, C. L. Ruiz, G. W. Cooper, K. D. Hahn, J. McKenney, A. C. Owen, G. R. 
McKee, G. T. Leifeste, D. J. Ampleford, E. M. Waisman, A. Harvey-Thompson, R. J. Kaye, M. H. Hess, S. E. Rosenthal, and M. K. Matzen, IEEE Transactions on Plasma Science 40, 3222 (2012)

74. G.R. Gathers, International Journal of Thermophysics 4, 271 (1983)

75. T. W. Chapman, Materials Science and Engineering 1, 65 (1966)

76. A. L. Horvath, Journal of Chemical Education 50, 335 (1973)

77. G. I. Kerley, ed., Equations of State for Titanium and Ti6A 14V Alloy, Sandia National Laboratories (2003) 